\title{
The Fibreculture Journal
}

DIGITAL MEDIA + NETWORKS + TRANSDISCIPLINARY CRITIQUE

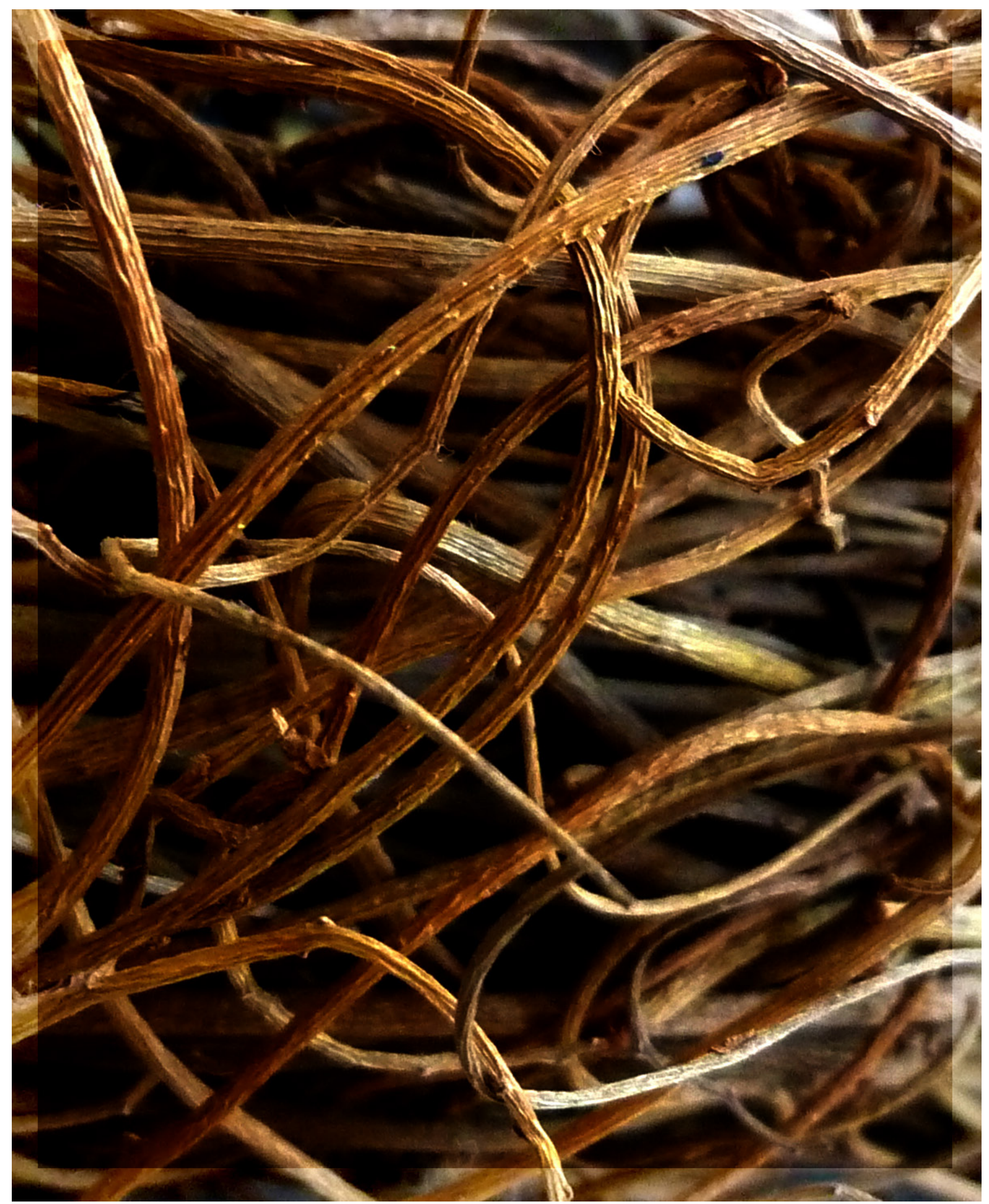

Issue $26: 2015$

Entanglements - Activism and Technology

Edited by Pip Shea, Tanya Notley, Jean Burgess and Su Ballard 
The LOCKSS System has the permission to collect, preserve and serve this open access Archival Unit

This Isuue of the Fibreculture Journal by The Fibreculture Journal Incorporated is licensed under a Creative Commons Attribution 4.0 International License.

\section{OPEN HUMANITIES PRESS}

The Fibreculture Journal is published by The Fibreculture Journal Incorporated in partnership with Open Humanities Press.

ISSN: 1449 - 1443, Published in Sydney, Australia

Fibreculture Journal Inc. in partnership with The Open Humanities Press 2013

The journal is peer reviewed as per section 4.3.4 of the Australian HERDC Specifications. 


\section{About the Fibreculture Journal}

The Fibreculture Journal is a peer reviewed international journal, first published in 2003 to explore the issues and ideas of concern to the Fibreculture network.

The Fibreculture Journal now serves wider social formations across the international community of those thinking critically about, and working with, contemporary digital and networked media.

The Fibreculture Journal has an international Editorial Board and Committee.

In 2008, the Fibreculture Journal became a part of the Open Humanities Press, a key initiative in the development of the Open Access journal community.

In 2012 the Fibreculture Journal celebrated ten years of open access scholarly publishing with the publication of its 20th Issue.

The journal encourages critical and speculative interventions in the debate and discussions concerning a wide range of topics of interest. These include the social and cultural contexts, philosophy and politics of contemporary media technologies and events, with a special emphasis on the ongoing social, technical and conceptual transitions involved. More specific topics of interest might include:

$::$ informational logics and codes

$\because:$ the possibilities of socio-technical invention and sustainability

$::$ the transdiscplinary impacts of new media technologies and events in fields such as education, the biosciences, publishing or knowledge management

$::$ information and creative industries, media innovation, and their critique

$\because:$ national and international strategies for innovation, research and development

$\because:$ contemporary media arts

$::$ new forms of collaborative constitution made possible by contemporary media

$\because:$ software and hardware develops in relation to the social

$::$ networks :: media change, convergence and divergence

$::$ the use of contemporary media in socio-technical interventions

The Fibreculture Journal encourages submissions that extend research into critical and investigative networked theories, knowledges and practices.

The Fibreculture Journal values academic scholarship in the field, and demonstrates this through the publication of refereed articles. The journal is fully supportive of Open Access communities and practices, and is committed to contemporary metadata provisions and uses. It is also open to expanded notions of scholarship which might include collaborative hypertexts, database compositions, and low-band electronic installations that experiment with the philosophy, politics and culture of information and communication technologies.

ISSN: 1449 - 1443 Published in Australia

Publisher: The Fibreculture Journal/The Open Humanities Press 2014

The journal is peer reviewed as per section 4.3.4 of the Australian HERDC Specifications. 


\title{
Editorial and Management Committees
}

\author{
editors
}

Su Ballard (University of Wollongong)

Glen Fuller (University of Canberra)

Andrew Murphie (University of New South Wales, Sydney)

contact editorial : fibreculturejournal@gmail.com

\section{journal manager}

Mat Wall-Smith (Independent Scholar, Sydney)

contact manager : fcjmanager@gmail.com

\section{editorial and management committee}

Su Ballard (University of Wollongong, Wollongong) Lone Bertelsen (Independent Scholar, Sydney)

Danny Butt (University of Melbourne)

Chris Chesher (University of Sydney)

Glen Fuller (Canberra University, Canberra)

Lisa Gye (Swinburne University of Technology, Melbourne)

Ross Harley (University of New South Wales, Sydney)

Adrian Miles (RMIT, Melbourne)

Lizzie Muller (University of Technology, Sydney)

Anna Munster (University of New South Wales, Sydney)

Andrew Murphie (University of New South Wales, Sydney)

Brett Neilson (University of Western Sydney)

Elena Razlogova (Concordia University, Montréal)

Ingrid Richardson (Murdoch University, Perth)

Ned Rossiter (University of Western Sydney)

Mat Wall-Smith (University of Wollongong)

Mitchell Whitelaw (Canberra University, Canberra)

\section{editorial board}

Belinda Barnet (Swinburne University of Technology, Melbourne)

Linda Carroli (Harbinger Consulting, Australian Network for Art and Technology)

Chris Chesher (University of Sydney)

Felicity Colman (Manchester Metropolitan University)

Melinda Cooper (University of Sydney) 
Kate Crawford (University of New South Wales, Sydney / Microsoft Research, Boston)

Sean Cubitt (Goldsmiths, University of London)

Michael Dieter (University of Amsterdam)

Sher Doruff (Amsterdam School of the Arts)

Pia Ednie-Brown (RMIT, Melbourne)

Mary Flanagan (Dartmouth College, New Hampshire)

Terry Flew (Queensland University of Technology, Brisbane)

Kelli Fuery (Chapman University, California)

Gary Genosko (University of Ontario, Canada)

Phil Graham (Queensland University of Technology, Brisbane)

Melissa Gregg (University of Sydney)

Maren Hartmann (University of the Arts, Berlin)

Robert Hassan (Swinburne University of Technology, Melbourne)

Larissa Hjorth (RMIT, Melbourne)

Teri Hoskin (Artist/electronic writing research ensemble. Adelaide)

Troels Degn Johansson (University of Copenhagen)

Paul Jones (University of New South Wales, Sydney)

Andrew Kenyon (University of Melbourne)

Julian Kucklich (Mediadesignhochschule, Berlin)

Elaine Lally (University of Technology, Sydney)

Geert Lovink (University of Amsterdam)

Niall Lucy (Curtin University, Perth)

Adrian Mackenzie (Lancaster University)

Lev Manovich (University of California, San Diego)

Thomas Markussen (Aarhus School of Architecture)

Graham Meikle (Stirling University, Scotland)

Catherine Mills (University of Sydney)

Esther Milne (Swinburne University of Technology, Melbourne)

Timothy Murray (Cornell University, New York))

Jussi Parikka (University of Southampton)

Simon Penny (University of California, Irvine)

Andy Polaine (Lucerne University of Applied Sciences and Arts)

John Potts (Macquarie University, Sydney)

Melinda Rackham (RMIT, Melbourne)

Philip Roe (Central Queensland University, Bundaberg)

John Scannell (Macquarie University, Sydney)

Sha Xin Wei (Concordia University, Montréal)

Kate Southworth (iRes Centre for Interactive Art and Design, University College, Falmouth, UK)

John Sutton (Macquarie University, Sydney)

Tiziana Terranova (Università di Napoli L'Orientale', Italy)

David Teh (National University of Singapore)

Nathaniel Tkacz (Warwick University)

Darren Tofts (Swinburne University of Technology, Melbourne)

Gregory L. Ulmer (University of Florida)

José van Dijck (University of Amsterdam)

Jill Walker (University of Bergen)

Shujen Wang (Emerson College, Boston) 


\section{The Fibreculture Journal:}

Issue 262015 Entanglements - Activism and Technology

Editorial:

Pip Shea, Tanya Notley, Jean Burgess.

Articles:

FCJ-188 Disability's Digital Frictions:

Activism, Technology, and Politics

Katie Ellis

Curtin University

Gerard Goggin

University of Sydney

Mike Kent

Curtin University

FCJ-189 Reimagining Work:

Entanglements and Frictions around Future of Work Narratives

Laura Forlano

Illinois Institute of Technology

Megan Halpern

Arizona State University

FCJ-190 Building a Better Twitter:

A Study of the Twitter Alternatives GNU social, Quitter, rstat.us, and Twister

Robert W. Gehl

The University of Utah 
FCJ-191 Mirroring the Videos of Anonymous:

Cloud Activism, Living Networks, and Political Mimesis

Adam Fish

Lancaster University

FCJ-192 Sand in the Information Society Machine:

How Digital Technologies Change and Challenge

the Paradigms of Civil Disobedience

108

Theresa Züger

Alexander von Humboldt Institute for Internet and Society

Stefania Milan

University of Amsterdam

Leonie Maria Tanczer

Queen's University Belfast

FCJ-193 Harbouring Dissent:

Greek Independent and Social Media and the Antifascist Movement

Sky Croeser

Curtin University

Tim Highfield

Queensland University of Technology

FCJ-194 From \#RaceFail to \#Ferguson:

The Digital Intimacies of Race-Activist Hashtag Publics

Nathan Rambukanna

Wilfrid Laurier University

FCJ-195 Privacy, Responsibility, and Human Rights Activism

Becky Kazansky

Tactical Technology Collective 
FCJ-196 Let's First Get Things Done! On Division of Labour and Techno-political Practices of Delegation in Times of Crisis

Miriyam Aouragh

Westminster University

Seda Gürses

New York University

Jara Rocha

Bau School of Design

Femke Snelting

Constant Association for Art and Media

FCJ-197 Entanglements with Media and Technologies in the Occupy Movement

Megan Boler

OISE/University of Toronto

Jennie Phillips

OISE/University of Toronto Bau School of Design

Practitioner Reports:

FCJMESH-005 Technology and Citizen Witnessing:

Navigating the Friction Between Dual Desires for Visibility and Obscurity 268

Sam Gregory

WITNESS

FCJMESH-006 From Information Activism to the Politics of Data

Maya Indira Ganesh and Stephanie Hankey

Tactical Technology Collective

FCJMESH-007 Our Enduring Confusion About the Power of Digital Tools in Protest

Ivan Sigal and Ellery Biddle

Global Voices 
Jillian C. York

Electronic Frontier Foundation

FCJMESH-009 Ranking Digital Rights:

Keeping the Internet Safe for Advocacy

Nathalie Maréchal

Ranking Digital Rights Project

FCJMESH-010 : Getting Open Development Right

Zara Rahman

the engine room

FCJMESH-011 : 'We don't work with video, we work with People':

Reflections on Participatory Video Activism in Indonesia

M. Zamzam Fauzanafi

Kampung Halaman 


\section{The Fibreculture Journal}

DIGITAL MEDIA + NETWORKS + TRANSDISCIPLINARY CRITIQUE

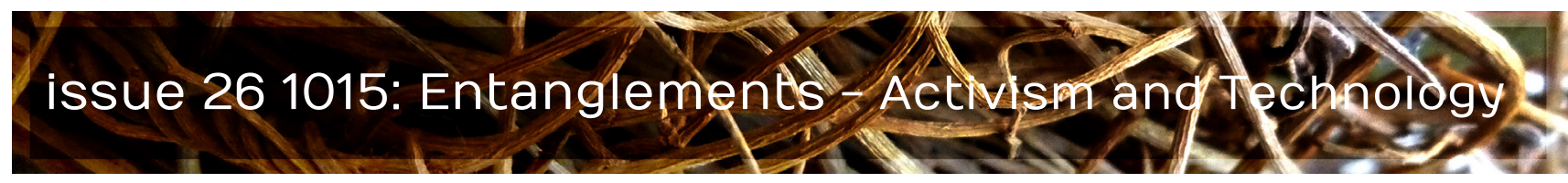

\section{Editorial:}

\section{Pip Shea}

Farset Labs

Tanya Notley

Western Sydney University

Jean Burgess

Queensland University of Technology

issue doi: $10.15307 /$ fcj.26

During the 2009 post-election protests in Iran, YouTube proved useful for raising awareness and mobilising people; but later, the Iranian government used these videos to crowd-source the identification of protesters.

Activists used Skype to communicate during the Egyptian uprising thinking it was safer than the terrestrial telephone system; however, when they examined files from the intelligence agency in the chaos after Mubarak's fall they learnt their Skype calls were being closely monitored by Egypt's security service .

One of the most circulated images appealing for public sympathy and money following the 2015 catastrophic Nepal earthquake turned out to be a ruse-an old image from North Vietnam-its circulation initiated by unknown people with unknown motivations.

These examples serve to remind us that while digital technologies are now deeply entangled with activist practices that are focused on contributing to social change, the philosophies and capacities embedded within these technologies often contradict, counteract, or challenge social justice and human rights aspirations-sometimes in unexpected ways that could not have been predicted. 
A decade ago these kinds of examples would have more than likely been used to support cyber-optimist and cyber-pessimist arguments-and these one-sided perspectives are still easy to find in academia, journalism and in public debate. But after at least a decade of widespread use of technologies for activism in countries all over the world we seem to have-at last-turned a corner; nuanced debates and discussions about activist-technology entanglements and their implications are far more common.

This issue was motivated by our shared desire to explore these entanglements with scholars and activists who are working within, experiencing, and researching the frictions caused by technologies when they are used for activism. We use the term 'friction' as Anna Tsing does-as a metaphor for the diverse and sometimes conflicting engagements that make up our contemporary world or what she calls 'zones of awkward engagement.' Tsing defines friction as 'the awkward, unequal, unstable, and creative qualities of interconnection across difference' that continually co-produce culture (Tsing, 2005: 4). Through an examination of frictions between aspirations and realities, between needs and constraints, a critical analysis of global connection is possible. In this way, the concepts of entanglements and frictions support us to explore the complex realities of co-dependent relationships between activists, technologies and the corporations who create them, in ways that support us to move beyond the old, dull and tired 'good' versus 'bad' technology narratives.

Past issues of Fibreculture have examined activist philosophies from angles such as social justice and networked organisational forms, communication rights and net neutrality debates, and the push back against precarious new media labour. Our issue extends this work by capturing the complexities associated with the use of technology in activist contexts, and offering insights into how practitioners, scholars, and the makers of digital and networked technologies do and might need to work more collaboratively and pragmatically to address social justice issues.

This issue includes ten academic journal papers as well as seven invited articles from practitioners who are working on the very front lines of activism and technology. This section from practitioners is a first for the Fibreculture Journal. These articles allow us to better understand the decisions made by organisations and activists who are leading debates, negotiations and discussions and from those who have most at stake because they depend on technology working and working well for activism.

By dwelling in between and within frictions and entanglements, the activist practices described and interrogated in the academic papers and practitioner articles that follow 
reveal tensions, weaknesses, and sites of contested power within fields such as international development, human rights, social movements, and community development. Articles explore how philosophies of technology and activism become enmeshed through struggle, acceptance, compromise, or submission (Tsing, 2005; Crosby and Notley, 2014); and the ways in which these negotiations speak to broader mythologies and tensions embedded within digital culture-between openness and control; political consistency and popular appeal; appropriateness, usability, and availability.

Labour activism and the consequences that flow from entanglements with technologies are emphasised in a number of essays in this issue. Katie Ellis, Gerard Goggin, and Mike Kent investigate disability activism movements, arguing that digital technologies work both for and against the disability justice project. Their focus on protests against welfare and work reforms in the UK reveal how disability activists, mainstream media, social media, and state apparatuses entangle to problematise the aspiration of being 'fit for work.' Other frictions arising from disability activism confronting technology-for example, between the philosophy of Universal Design and the needs of specific individuals-are also investigated. Laura Forlano and Megan Halpern use speculative and participatory design methods to elucidate the role of emerging technologies such as automation, artificial intelligence, and robotics in narratives concerning the future of work. This promotion of 'material deliberation' is intended to encourage labour activists to engage more deeply with technologies.

Software cultures that elevate alternative and activist practices over other forms of participatory media activities also feature as a recurrent theme. The nuanced material politics of alternative social media projects is the focus of Robert Gehl's piece. His discussion about how developers are 'critically reverse-engineering' Twitter reveals an ongoing friction of activist projects: that of people with different evaluative frameworks working towards similar goals. A detailed discussion by Adam Fish about the politics of 'mirroring' as practiced on videos by the hacktivist network Anonymous is another take on activist software cultures. Drawing on Haraway (1992), he situates these mirroring activities as having the effects of 'diffraction'-a mapping of interference rather than replication or reflection, as the name suggests-that serve to visually map a contestation over networked visibility.

Also touching on software cultures, Theresa Züger, Stefania Milan and Leonie Tanczer offer an overview of emergent digital civil disobedience practices-such as 'cloud protesting'-discussing the frictions that arise due to anonymity, multiple agendas, and loose affiliations. Their article reminds us of the often-porous boundaries between fields that spark tactical media activities, politically motivated artistic interventions, and more 
traditional modes of civic subversion; but calls for new articulations of civil disobedience focused on digital dissent. Sky Croeser and Tim Highfield extend the theme of civic dissent through their study of the Greek antifascist movement. Their article stresses how events and dynamics must be considered in the context of several interrelated trends affecting Greece, and argues that this movement is created and constantly recreated through spaces, events, social media technologies, and unexpected and temporary alliances. We are also reminded that people, who do not consider themselves activists, or even part of a movement, perform many of these associated activities.

Further investigations of the affordances and consequences arising from the use of social media for articulating dissenting discourses can be found in Nathan Rambukkana's study of race-activist hashtags. Frictions emerging from activists using hashtags that might be filtered by corporations are explicated through the cases of \#RaceFail and \#Ferguson. Facebook's 'algorithmic filtering' of \#Ferguson is situated as a case in point, illustrating how social media filtering practices can affect whether or not we see controversial, fractious, or political content. Philosophies of autonomy and privacy in relation to digital technologies are explored in Becky Kazansky's article. She problematises the framing of privacy as a predominantly individual responsibility, exploring the origins of this framing, and she discusses ways in which this burden of responsibility might be shifted away from activists and vulnerable groups.

Entrenched social hierarchies and their effects on the shaping of activist technology uses and practices are another shared theme of this issue. Miriyam Aouragh, Seda Gurses, Jara Rocha and Femke Snelting discuss how the development of supposedly appropriate technologies are compromised by time pressures and needs for efficiency during moments of urgency. They highlight how the justification of these processes as divisions of labour, paradoxically reproduces traditional hegemonic dynamics. The shaping of activist practices by stereotypical gender roles in the Occupy Movement are investigated by Megan Boler and Jennie Phillips in their article. This critique of gender bias is part of a wider exploration of the paradox of activists using corporate-owned software platforms.

Across the practitioner articles in FCJ MESH we can identify a number of particularly pertinent themes. The struggle to support the need to be both private and visible across digital networks-sometimes at the same time-is a source of friction addressed by Sam Gregory in his article about the politics and practices of citizen witnessing. Maya Ganesh and Stephanie Hankey describe how surveillance, data sharing, aggregation, and the storage of metadata have different impacts for different actors and how activists can be a group at-risk of exposure. They also make us aware of issues surrounding corporate attempts to make things seemingly 'frictionless' by hiding the functionality of technologies. 
Ivan Sigal and Ellery Biddle describe how the realities, efforts, stories, and needs of some activists are obscured when the media misrepresent the emancipatory potential of digital technologies (we would argue that same can be said when researchers do this). Another messy entanglement affecting activism and technology are the realities of free speech ideologies often embedded within social media dynamics-a subject tackled by Jillian C. York. Nathalie Maréchal describes a project exploring the opportunities and challenges associated with evaluating the world's internet and mobile companies on policies and practices related to free expression and privacy in the context of international human rights law. Through a study of the Open Development Movement, Zara Rahman reveals tensions between the aspirations of activists and the needs of those they are trying to help. Finally, Zamzam Fauzanafi reminds us how the dynamics of community organising continue to shape community projects that focus on creative uses of technology.

In the late nineteenth century, pragmatist philosopher William James wrote, 'There can be no difference which doesn't make a difference,' to stress the importance of thoughts and theoretical positions having practical effects (James, 1898). This rousing sentiment is a seductive call to action, but fails to consider how 'making a difference' is perceived, measured, or publicised through different evaluative lenses. In contrast, the nuanced stories attached to diverse activist practices discussed in this issue show how ongoing frictions between philosophies and practices continue to deliver outcomes that make a difference to some and not others. Disagreements about what should be valued will remain a variable in projects that use technology to challenge dominant social, cultural, and economic paradigms. However, this collection builds a compelling case for using the energy derived from frictions-produced by activists with different realities, needs, and evaluative frameworks-to inspire and develop action that ensures technologies work and work well for activism.

Frictions will continue to emerge as global technologies work to universalise needs, experiences, and contexts (Crosby and Notley, 2014). Accepting frictions when activism and technology come together also allows us to explore 'the messy and surprising features' of global encounters across difference that 'should inform our models of cultural production' (Tsing, 2005: 3). While we can be cynical about the likely consequences of uneven power dynamics embedded within these frictions, the more hopeful perspectives that emerge in this issue suggest that for this co-production to be productive-and for activist uses of technology to flourish in different contexts-negotiations and compromises must be made between regulators, technology producers, and activists if we are to transform friction into meaningful actions that are capable of supporting positive social change. 


\section{Biographical Note:}

Pip Shea is a digital media researcher, designer, and educator. She investigates how digital cultures and new organisational forms are shaping creative, civic, and activist practices. She is a director at Farset Labs hackerspace and technology charity in Belfast, Northern Ireland.

Tanya Notley is a Lecturer in Internet Studies and Digital Media in the School of Humanities and Communication Arts at the University of Western Sydney. As a researcher, board member and adviser, Tanya works closely with a number of NGOs to design and implement communication technologies.

Jean Burgess is Director of the Digital Media Research Centre at Queensland University of Technology. She researches the everyday uses and politics of social and mobile media platforms, as well as new digital methods for studying them. Her books include: YouTube (Polity Press, 2009), Studying Mobile Media (Routledge, 2012), A Companion to New Media Dynamics (Wiley-Blackwell, 2013), and Twitter and Society (Peter Lang, 2014).

\section{References}

James, William. Philosophical Conceptions and Practical Results (Berkeley, Ca.: University Press, 1898).

Haraway, Donna. 'The Promises of Monsters: A Regenerative Politics of Inappropriate/d Others in Cultural Studies', in Lawrence Grossberg, Cary Nelson and Paula A. Trechiler (eds). Cultural Studies (New York, NY: Routledge, 1992), 295-337.

Crosby, Alexandra and Tanya Notley. 'Using Video and Online Subtitling to Communicate Across Languages from West Papua', The Australian Journal of Anthropology 25.2 (2014): 138-154.

Tsing, Anna. Friction: An Ethnography of Global Connection (Princeton, NJ: Princeton University Press, 2005). 


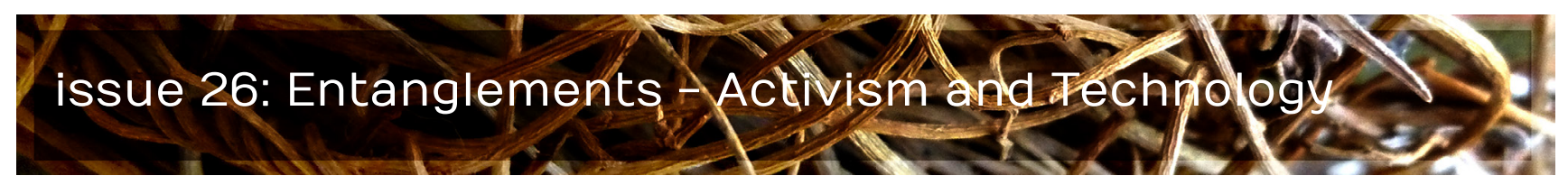

\title{
FCJ-188 Disability's Digital Frictions: Activism, Technology, and Politics
}

Katie Ellis

Curtin University

Gerard Goggin

University of Sydney

Mike Kent

Curtin University

\begin{abstract}
:
This paper argues that disability activism and politics can be seen as paradigmatic for the wider debates on the philosophies, forms, and formats embedded in technologies. Firstly, the paper discusses disability activism movements that use digital technology to intervene into digital technology, especially in the social media area. In both cases, activists seek to use the platforms themselves to argue for, and obtain, greater accessibility, customisability, and configurability, of the platform for users with disabilities. Yet there is a clear clash between philosophies embedded within these social media platforms, and the aspirations and values of many of the activist strands of disability. Secondly, the paper explores the use of digital technologies by disability activism movements in relation to welfare and work reforms-notably the UK struggles in the 2010-2014 periods. Here disability activists have made extensive use of digital media technologies, and we discuss their innovations, and the lessons they offer.
\end{abstract}

doi: 10.15307/fcj.26.188.2015 


\section{Introduction}

Increasingly, disability is acknowledged as a key part of society, public and private spheres, and everyday life. Moreover, disability has achieved notable recognition and endorsement as an area of inequality, oppression, and discrimination that requires concerted global and local action.

We see various markers of this transformation in the social relations of disability. In the legal realm there is the enactment of the United Nations Conventions on the Rights of Persons with Disabilities (Arnardóttir and Quinn, 2009; Flynn, 2011), and the cumulative effect of many important laws and regulations enacted by governments around the world (Francis and Silver, 2000; Waddington, Quinn and Flynn, 2015). Related positive developments include greater visibility and potency of people with disabilities in public spheres and counter public spheres.

There is increasing acknowledgement of the specific gender, class, race, and sexuality dimensions of disability (Kulick and Rydström, 2015; McCruer, 2006; Samuels, 2014). Also notable is the dawning mainstream recognition for efforts to understand and transform the situation of indigenous people with disabilities (Soldatic, Spurway and Meekosha, 2014). Finally overdue attention is being paid to disability in the majority world, often termed the 'global south', or 'developing world' (Grech and Soldatic, 2015; Soldatic and Meekosha, 2014). Questions of survival and the precarity of existence are raised starkly in these countries, for people with disabilities especially those belonging to the less wealthy or powerful groups-those many people who are among the most marginalised and excluded (Erevelles, 2011).

Much oppression and many challenges remain concerning disability justice. There is a precariousness of living with a disability in contemporary Western societies. While there have been significant initiatives on work and labour issues (Bruyère and Barrington, 2012; Heymann, Stein and Moreno, 2014), the realities and stereotypes of disability are clearly visible in debates on welfare policy, work, and disability support (Lindsay and Houston, 2013; Marin, Prinz and Queisser, 2004; Roulstone, 2012; Soldatic, Morgan and Roulstone, 2014).

These are some of the important contexts for the groundswell of activism in relation to disability. Such activism, and indeed the contours of disability in everyday life, has intimate 
and consequential connections with technology. Digital technology provides new kinds of tools, support, and power for people with disability. Thus digital technology is often vital for everyday life, but also for engagement and participation in contemporary politics, culture, and media (Blume, 2012; Mills, 2011). A specific kind of activism has emerged here that focusses on the shortcomings, problems, and inaccessibility of digital technology for many people with disabilities (Blanck, 2015; Ellis and Kent, 2011; Goggin, 2014; Goggin and Newell, 2003; see also Padovani \& Calabrese, 2014). However, there is a much broader perspective needed here. To start with, it is important to note that disability has very interesting relationships with technology (Hickey-Moody and Wood, 2008; Moser, 2005), something that makes sense if we acknowledge the social nature of disability (Goggin and Newell, 2003; Roulstone, 1998). Various modern social imaginaries as well as materialities of disability involve technology (Goggin and Noonan, 2006), just as much as they are premised on activist and transformative accounts of disability movements. Disability and technology can be seen as entangled (Thomas, 1991), whereas they are often regarded as quite separate-for instance, in discourses of web accessibility (Adam and Kreps, 2009), or in assistive technology. There is a growing situation of interdependence for many people with disabilities-as in social life generally-whereby life without some kind of technology seems unachievable and unimaginable (Goggin and Newell, 2006).

The kinds of digital technology we discuss here are eminently global in their nature, providing the international connections that undergird and make possible the social transformations of disability-as well as the universal claims, for instance, of human rights. While discourses of globalisation and technology in the 1990s in particular saw motion and global connection as increasingly trouble-free and friction-less, the reality is a contradictory, messy, materially situated process (Tsing, 2005: 5-6). Rather than there being a binary, for instance, between 'access' and 'non-access', we see friction in operation across the global field of disability and technology-especially in relation to disability activism.

Accordingly, in this paper we use this disability analysis and theorisation to reflect back upon the general problematics of how technology and activism are sutured together. The two main case studies of the paper proceed to explore these two tightly connected aspects of disability activism and technology.

In the first part of the paper, we take up the long-running struggle of disability activists over accessibility of digital technology. We start with highly publicised movements around the accessibility of Facebook and Twitter. In both cases, activists seek to use the platforms themselves to argue for, and obtain, greater accessibility, customisability, and configurability, of the platform for users with disabilities. Yet there is a clear clash between 
philosophies embedded within these social media platforms, and the aspirations and values of many-stranded disability activism.

The second part explores the use of digital technologies by disability activism movements in relation to welfare and work reforms. We look particularly at the British disability movement's struggles around welfare reforms since the election of the Conservative government in 2010 and the 2012 Paralympic Games, where disability activists have made extensive use of digital media technologies. A fascinating instance is also found in the work of artist Liz Crow, who has used social media to facilitate conversation and debate around her installation work that exposes and critiques disturbing and oppressive aspects of the UK disability welfare forms.

\section{Disability Activism Confronts Technology: Beyond Accessibility}

As we have discussed, disability activism is a fascinating and important case of creating and realising the 'democratic affordances' of digital technologies (Goggin, 2013). For the most part, such technology-enabled disability activism has not been chronicled, theorised, or debated in the burgeoning literatures on social movements, social media and other digital technologies, and activism. Acknowledgement, incorporation, and exploration of disability activism's contribution to the ideas, practices, and repertoire of digital activism more generally is a project well underway in the social movements themselves-but scarcely commenced in research and scholarship.

This disability technology activism has involved a range of actors-activists with disability, allies, technology experts and developers (with and without disability), civil society organisations, governments, and human rights practitioners. Effectively such activism has identified, debated, and challenged the philosophies in digital technologies for many years-especially in the period of the Internet's widespread diffusion from the 1990s onwards. Following the lead of Judy Wajcman in her classic 1991 book on the politics of technology, Feminism Confronts Technology we could easily dub this movement 'disability confronts technology' (Wajcman, 1991).

Broadly speaking, this movement is better known as focused on Internet accessibility for people with disabilities. It is best developed and most widely familiar in the area of the World Wide Web, due to the efforts of the World Wide Web Consortium (W3C) Web Accessibility Initiative (WAI) (Brewer, 2001). The W3C WAI commenced in 1997. Famously Sir 
Tim Berners-Lee called for the web to be globally accessible-a universal medium. This is a call he has renewed in the commemoration celebrations in 2014, suggesting the Internet is at a crossroads. Berners-Lee sees accessibility of the Internet for people with disabilities as a core part of realising his broader vision for the universal accessibility of the Web and Internet, and now mobile and other associated technologies. The W3C WAI, and the wider movement centering on the accessibility of digital technology seek to contest a particular philosophy of technology, especially around invention, design, and creativity.

Namely, that technology designers should be free to write the code, design, construct, and implement the technology they wish. For their part, users can choose to adapt and fit their desires to the platform, or, alternatively, seek another platform. The accessibility movement counterposes a different philosophy of technology, with a core argument that accessibility should always be part of the innovation and design process of digital technology.

Perhaps the most influential strand of this counter-philosophy is 'universal design', the idea that technology should, as far as possible, be designed for the widest population possible (Preiser and Ostroff, 2001). If one designs for disabled users, the argument goes, then the end result will be technology that is of more use (and more accessible) to a wider range of users.

A concrete example may be found in contemporary multimedia, which is often not accessible for particular groups, or individuals. To address this, sub-titling online videos for Deaf people or hard-of-hearing users, for instance, means that users in noisy environments, or who need to mute their device volume, or for videos where users find the language hard to understand can be assisted also. It also allows users to search within videos.

Universal design has been adopted in mainstream documents and practices, including the UN CRPD. It is important to note that universal design, especially in its simple forms, poses its own problems for disability activism and broader concepts of disability (see Goggin and Newell, 2003; and Imrie and Luck, 2014). There is a tension, for instance, between the universalising impulse-and the need to understand, address, and support the oftencontradictory needs and expectations of situated, specific groups and individuals.

In many ways, the ideas of digital accessibility have their roots not just in confronting discrimination and exclusion, but also in philosophies of user-centred design, across a range of disciplines and settings, including the Human-Computer Interaction ( $\mathrm{HCl})$ field. 
A cognate idea is the longstanding ideal of participatory design: the claim-in weaker or stronger versions-that users, and others affected by technological systems, should have some stake and say, some genuine control, in their design and implementation (Schuler and Namioka, 1993).

Web accessibility has focussed on large organisations, commercial, NGOs, and governments and their agencies. It has relied upon governments to enact laws that require web accessibility. Governments have also been viewed as important leaders in accessibility - with a duty, for instance, to ensure that government departments and agencies are good role models in making their websites, and services, accessible to all citizens and users, especially those with disabilities.

Suffice to say, the progress of web accessibility-even in its most basic requirements-has been slow. Some critics of web accessibility have made fundamental critiques also, arguing that its framing, discourses, and actors continue to perpetuate a narrow, oppressive view of disability (see Lewthwaite, 2014; for a critique of disability access in general, see Titchkovsky, 2011).

In particular, a tension exists between the socio-political use of social media as a platform for activism and 'the commercial interests of the platform owners' (Youmans and York, 2012). This is something that has been widely acknowledged and discussed elsewhere, in critiques from different perspectives (such as Andrejevic, 2007; and Fuchs, 2014a \& 2014b). For instance, Facebook is recognised as a potential site of empowerment and social inclusion for people with disability as widely discussed across disability culture and activism, and recognised in some literature (see Haller, 2010; Hollier, 2012). Yet the platform itself has a vexed relationship with accessibility (Ellis and Goggin, 2015).

For instance, in 2007, Andrew McKay, a student with vision impairment prompted an American Foundation of the Blind accessibility overhaul of Facebook following his activism, via the Facebook page The Official Petition For a More Accessible Facebook (Ellis and Goggin, 2015; Ellis and Kent, 2011; Haller, 2010). The page attracted over 1500 members and urged Facebook to correct seven accessibility errors. While Facebook did pay attention and implemented accessibility changes in consultation with the American Foundation of the Blind subsequent updates have again compromised access for users with vision impairment (Holler, 2012). 
Similar accessibility issues have been identified with the Twitter platform where users with vision impairments have experienced difficulties accessing the text based medium with screen readers (Ellis and Goggin, 2014; Ellis and Kent, 2010). When Twitter initially ignored the issue, independent web developers created accessible Twitter third party apps such as Dennis Lembree's 'Accessible Twitter' (later renamed EasyChirp). However, Twitter has since announced ongoing accessibility improvements to the Twitter app (Twitter, 2013; Twitter, 2014).

At a deep level, online access to a text is linked to power and control. Simply put, Facebook does not want to refer its users to other sites, it wants them to stay within the Facebook ecosystem for monitoring and advertising, thus it will embed videos from other sites within its own news stream rather than refer users away to another site. Google adopts a similar model. However these practices come with certain assumptions about what is 'normal' in the users of these services. While the Internet has provided an avenue of increased access to information and activism, there are still barriers, often unintended, that prevent people with disabilities from accessing this material. An urgent area for improvement is routine provision of video captioning and audio description of material to provide modalities for access.

A number of disability theorists highlight a paradox between the potential for increased social participation via the Internet for people with disability and the continuing isolation they experience as a result of inaccessible platforms and interfaces. For example, Sourbati argues that as communications are moving to an all-digital environment, public policy needs to be rethought (Sourbati, 2012: 571). She describes access to ICTs and the services provided by them as critically important arguing that that not having access to these "can be seen to prevent individuals from fulfilling active roles in society' (575). We can sharpen up the edges of this discussion, when we consider activism and political participation. If applications, interfaces, devices-in short, affordances of digital technology-are not accessible, open, and configurable, when activism often requires such technology, then there is a clear problem.

Disability activism concerning technology, then, has strong links with broader debates about rights to technology, such as the renewed movement focused on 'Internet rights', and 'Internet freedom', which recognises that democracy is entangled with technologyand that people's capacity to avail themselves of technology is foundational. If the platforms are not supportive of such aspirations, not only is it difficult for 'communicative capitalism' to function (Dean, 2009), but any emancipatory or transformative political project is stymied. 


\section{UK Welfare Reform Protests: Disability Activism with Digital Technology}

There are a number of striking contemporary cases of people with disabilities and how their allies use digital technology to organise protests aimed at improving and transforming their lives-and to broadly engage in political, cultural, and social participation.

In broadly similar ways to those canvassed in the online activism literature (Boler, 2008; Dahlgren, 2013; Lievrouw, 2011; Meikle, 2002; Ratto and Boler, 2014), digital technology is being widely used to organise physical protests to raise awareness of disability rights issues. Such technology is relied upon during protests for organisation and co-ordination. Increasingly also through social media, digital technology serves as a way of publicising the protest action. It is both a substitute for mainstream media and a way of better connecting to, and drawing the attention of, mainstream media. Digital technology offers a way for those are unable to participate or be co-present in the designated places or sites of protest to still be involved. Finally, there is the still evolving area of 'purely' online activism-that is, activism largely based, or decisively, based online-what Zizi Papacharissi calls the 'agonistic pluralism of online activism' (Papacharissi, 2010: 157ff).

So far, so good. However, as we have already signalled in our preceding discussion of the accessibility and disability struggles over technology (under the watchword of 'disability confronts technology'), there are significant contradictions at play here. As we have argued thus far, this kind of friction between disability and the dispositions of technologies is not just a blockage or problem; rather it is a rich source of social action, ideas, and reflections that opens up into the larger problematic of political beings, participation, justice, and technologies, framing these against a broader horizon.

To unpack this, it is first important to say that while attention is often accorded to disability protest associated with digital technology, disability activism has long genealogies and debates (Chouinard, 1999; Fleischer and Zames, 2011; Newell, 2006; Oliver and Barnes, 2012; Sandell, Dodd, and Garland-Thomson, 2010; Shapiro, 1993). It is a big area, still requiring histories, research, and debate, but let us indicate some significant moments and tendencies in disability activism. Originally drawing inspirations from dissent and resistance, such as struggles of the women's suffragists before the First World War, or the protest movements of the 1960s (Shakespeare, 1993), disability rights protests were highly visible in the 1970s-for example in New York over accessible public transport, and over regulations around Section 504 of the Rehabilitation Act (Harris, Owen and De Ruiter, 2012). The relationship of disability activism to larger histories is highlighted in the often 
remarked story of wheelchair users in New York receiving help from the revolutionary group Weather Underground to dynamite kerbs that were not modified for wheelchair access (Pelka, 2012: 445, 593; Shakespeare, 1993). New kinds of disability activism have emerged around new categories and concepts, not least cognitive impairments, mental health and psychiatric disabilities, episodic and chronic conditions, and responses to genetics and biopower (Hughes, 2009; Kelly, 2013; Snyder and Mitchell, 2001; Scotch, 1988; Shakespeare, 2015).

These kind of larger frameworks for disability activism and protest movements help us to situate the way that, in recent times, digital media has been deployed to great effect. Digitally-enabled and inflected protest is a global phenomenon, of which little research exists (an exception being the research of Trevison 2013, 2014, and 2015). To explore the forms such technology interdependent citizenship takes, here we will focus on the UK protests that arose in response to the cuts made to disability benefits by the conservative government, particularly, leading up to, and during, the 2012 Paralympic games in London. As leading disability activist and artist Liz Crow nicely put it:

Timing a season of protest to coincide with the height of the Paralympics and benefits coverage, they sought to draw maximum press coverage of their campaign for justice and human rights for all disabled people in a time of austerity and welfare cuts ... In doing so, they showed what they were made of (Crow, 2014: 179).

These protests brought the use of digital communications technology to the fore, specifically to enable and support this political expression by people with disabilities in the face of severe cuts to disability support programs being made by the Cameron conservative government.

Leading up to the London Olympics were the National Day of Action protest on 24 January 2011 and the larger Hardest Hit protest of 11 May 2011. Both events had a well-documented online presence that was used to promote the events beforehand, and help mobilise and organise the protests (Preston, 2011). The first protest challenged a key aspect of the UK welfare reforms - the changes in how people with disabilities were assessed by the government as either 'fit for work' or 'unfit' and eligible for government welfare assistance. The protestors targeted Atos Origin, the company contracted to carry out the 'fit for work' assessments as part of administering the government's changes to disability payments. Atos present themselves as follows: 
We work closely with government to support its welfare reform agenda: 'helping people move into and progress in work, while supporting the most vulnerable' (Atos, 2015).

This is not a view shared by the disability activists opposing the reforms, criticising the pivotal role Atos played-and how the company acted. Atos Origin was also a sponsor of the Paralympics and the company would be a focus of protest again as the Paralympics ran the following year (Morse, 2012). The Hardest Hit protests' ability to mobilise and organise online drew between 3000 and 8000 protesters and brought together 200 separate disability rights organisations (Preston, 2011).

There are a number of platforms that help both organisers and participants in protest actions during protests. The use of mobile media to mobilise demonstrations was famously pioneered as part of the popular protests that saw President Estrada ousted from power in the Philippines in 2001 (Coronel, 2001; Pertierra et al., 2005). Since then the advent of smart phones and social media has greatly facilitated communications in these contexts, from the use of Twitter on mobiles, to more dedicated platforms such as the Sukey app that assists protesters avoid being 'kettled' (confined to small area during protest) by police in London (Geere 2010), or by mobile phone operated drones used by protesters to follow police activity (Ackerman, 2011).

While people with disabilities make use of these different affordances while marshalling and participating in protests, these platforms can also be used to both interact with, and circumvent, the mainstream media. So, for instance, in January 2012 in an action organised by Disabled People Against Cuts, a number of protesters in wheelchairs chained themselves together across Oxford Circus in London, bringing traffic to a halt for a number of hours. In this protest there was a heavy use of social media by people involved in the protest and the event was well documented on YouTube. This protest action reprised a famous previous protest. In 1990 CAT (Campaign for Accessible Transport) had previously blocked Oxford Street with a similar line of people in wheelchairs chained together. In 1990, the police had trouble dealing with wheelchair users while making arrests. Add to which, proceedings against many of the protesters were dropped when the courthouse where they were to be heard proved inaccessible (Shakespeare, 1993). This was grist to the mill for the protestors, as Allan Sutherland, press officer for CAT, remarked at the time: 'CAT was effective because its demos were well-organised, its message was simple, it provided good photo opportunities for the media and it had public support' (quoted in: Peck, 2010). Similar problems for police in dealing with people with disabilities were evident more recently in Australian protests when police were ill-equipped to arrest wheelchair users protesting cuts to disability coverage by the $A B C$ (Morton and Baxendale, 
2014). This had been an issue previously in the well-documented case of Jody Mclntyre being knocked from his wheelchair by police in the UK 2010 student fees demonstrations (Taylor, 2010).

Unlike the CAT protest, the prospect of media friendly images did not draw much attention to the 2012 Disabled People Against Cuts protest. However, the availability of YouTube videos through social media was used by the mainstream media, particularly The Guardian newspaper, to report the story. Similarly the police assault on Jody McIntyre became mainstream news after traditional media sources picked up video posted to YouTube through social media.

While people in wheelchairs may present a challenge for the police, actually attending physical protests also presents challenges for people with disabilities. There are a number of impediments including the costs of travel, and practical logistics of attending an event for a prolonged period of time including working with carers, toileting, and physical exhaustion, and in the case of these protests in the United Kingdom, the fear of being seen at the protest by people from the Department of Works and Pensions and then be declared 'fit for work' and the subsequent loss of benefits (Gentleman, 2011). Butler (2012) notes concerns that traditional methods of disability activism appear to be diminishing but that social media activism is on the rise, and makes the specific suggestion that this rise can be attributed to the UK government's welfare reforms.

Coinciding with the vote on welfare reform in the UK, Twitter, in particular, became an increasingly important forum for disability activists from early January of 2012 (Ryan, 2014). A number of disability activists used the platform to report on the hidden opposition to the government's plans to reform disability benefits. A coalition of 'sick and disabled people, who came together through social media' released the Responsible Reform report online and promoted it through Twitter. The report which became known online as the Spartacus report was 'entirely researched, written, funded and supported by disabled people' with information obtained through Freedom of Information requests. Campbell (2012) detailed that during the government's consultation period:

- 98 per cent of respondents objected to the qualifying period for benefits being raised from 3 months to 6 months.

- 99 per cent of respondents objected to Disability Living Allowance no longer being used as a qualification for other benefits. 
- 92 per cent opposed removing the lowest rate of support for disabled people (Campbell et al., 2012).

The activists argued that this information was kept from both the public and MPs debating the issue. Three months prior to the release of this report and associated social media attention, physical protests across the United Kingdom were called to protest against the reforms. This protest was the most attended of any disability related protest, and was held across 14 different locations (BBC, 2011) yet was largely ignored by the mainstream media (Butler, 2012). Despite the high attendance, a number of activists were unable to attend due to the effects of their impairments and the inaccessibility of the public transport system. As Ellen Clifford explains,

Disabled people have taken social media and made it into their own medium, where they can have a voice on equal terms with their non-disabled counterparts, something not often afforded by society as a whole...The computer provides a freedom for those with disabilities, it is much easier to protest online than in the centre of London when the Tube is not accessible (quoted in: Ryan, 2014).

This is also emphasised by activist David Gillon:

Many of us wouldn't be able to campaign at all without social media ... I barely get out of the house, and l've given up going into London at all, it's just too exhausting with my pain-based disability. No matter how many marches on parliament are called, I'm physically excluded by the realities of disability, and that's true for so many disabled people. Social media lets me campaign while lying flat on my back if I can't sit up, never mind march on parliament (quoted in: Ryan, 2014).

Social media did not just afford people with disabilities the opportunity to participate in the protest; it garnered mainstream media attention for an important disability issue that had, until it trended on Twitter, been ignored. Sue Marsh, an activist who contributed to the authoring of the Spartacus report explains the significance of social media:

For sick and disabled people campaigning, social media has been revolutionary ... It's been a magic bullet. It's given us political influence, media respect 
and international impact. I can't think of any [other] way sick and disabled people could have done what we and all of the campaign groups together have achieved (quoted in: Ryan, 2014).

The Twitter hashtag \#spartacusreport was able to make use of the affordance offered through the network. Initially it was used by an established network of disability activists to promote and disseminate the report's findings before it was picked up and retweeted by Stephen Fry to his three million followers and then further distributed through political figures Lord Prescott and Alistair Campbell, and then Billy Bragg, Val McDermid and Julie Hesmondhalgh. On the back of this support the hashtag top trended on the network. This lead to the department for work and pensions being drawn into the Twitter conversation itself to put out its justifications on the proposed reforms through using the same hashtag. On the back of the ensuing publicity of this online political action later in the week a number of these proposed reforms were the defeated in the House of Lords (Butler 2012).

More traditional web sites like that of Disabled People Against Cuts, the organisation which coordinated the 2012 Oxford Street protests, also play a complementary role to those physical protests by collating and distributing information that supports the protesters' message. Similarly The Broken of Britain website plays a crucial role as a place for online protest. The site curates social media, bringing together YouTube videos, blogs and Twitter to protest at the consequences of cuts to disability benefits in the United Kingdom (Gentleman, 2011). Kaliya Franklin one of the administrators of the site says she does not see the role of the site as to replace physical protest, but rather to act as a complementary form of protest (quoted in Gentleman, 2011). Another website that has been important is the Black Triangle Campaign's list of 'UK Welfare Reform Deaths':

> Updated tragic list of welfare-related deaths of UK's sick and/or disabled people. This is the tip on the iceberg:

They shall be remembered forevermore. Avenge the dead. Resuscitate the living. We must fight on for freedom from Westminster's murderous policies and support the struggle of all Britain's sick and/or disabled people simultaneously (McArdle, 2014).

As these recent UK disability protests develop, we also see elements of offline and online protest brought together in novel ways. Following the 2012 London Paralympics, a number of disability activists noted the pressure on people with disabilities to be 'superhuman' (Tracey, 2013; White, 2013). Liz Crow, a writer-director working with film, performance, audio and text, was one of those highlighting this issue. Crow argued that there was 
intense pressure 'to be a kind of superhuman when you're in public. And if you're not that, if you're a [benefits] claimant, then you're often cast as a scrounger' (quoted in: Tracey, 2013). Underpinned by a four-year NESTA (National Endowment for Science, Technology and the Arts) fellowship, Crow has explored ways to combine her creative practice and political activism. She comments that despite a long career in disability activism she had two lives - one public and one she kept private:

For about 30 years, I've been aware that I operate in two starkly different modes ... One is public, where I try and come across as energetic and animated and engaged and good at what I do. It's a way of being that's approved of socially. But what people don't see is the other side, where I spend most of my time at home, a great deal of it lying down in my bed. That's in order to prepare for the public thing, and to recover from it. I've always kept that hidden because it feels dangerous to make it public. It feels like l'd be misinterpreted and people won't see me as the whole person that I am (quoted in: Adewunni, 2013).

In protest against welfare benefit overhauls occurring in the United Kingdom, Crowe featured in Bedding Out, a 48-hour long disability performance piece at the Edinburgh Fringe Festival. In Bedding Out-a combination of art, performance and social mediaCrow wanted to show that disability is more complicated than the continuum of superhuman versus scrounger presented by the mainstream media (Macrae, 2012; Tracey, 2013). For disability, and other issues, Crow shows that there is a space in the middle.

Crow describes the performance on her website as a taking her 'private bed-oriented life and placing it in the public arena for all to see over a 48-hour period in order to show that what many see as contradiction, or fraud, is simply the complexity of real life' (Crow, 2012). The performance invites members of the public to join in on 'bedside conversations' which see them 'gathering around the bed to talk about the work, its backdrop and its politics, while those unable to attend in person are invited to take part virtually, through social media' (Crow, 2012).

The show was commissioned to be a part of the Disability Art's Online Perspectives project-the performances in this project were designed to 'spark conversations and debate about the Creative Case for Diversity' (Crow, 2012). By ensuring that those who were unable to attend the installation physically could still participate in the "bedside conversations', social media was integral to the performance. When a version of the performance was staged the previous year, many people contacted Crow to say 'they 
felt represented for the first time, and would like to be there but had their own bed-life. It's a life that's lived in a fair degree of isolation, and social media is breaking down the isolation' (Adewunni, 2013). Indeed the performance given in Salisbury was 'was watched on livestream by more than 9,750 people in over 50 countries' (Crow, 2012).

Emma Tracey, a writer for the BBC disability website Ouch!, discusses the production in terms of Crow's willingness to show her 'most-disabled self', something she had previously kept private as it as 'not socially acceptable' (Tracey, 2013). The Twitter hashtag used for the Salisbury run was active two weeks prior to the show and it began to build a community even before the performances; 'a community of people with bed lives of their own' (Tracey, 2013). The \#BeddingOut hashtag has been taken up as a call to arms-Tweets go beyond the installation piece and address the issues surrounding disability rights in the United Kingdom.

As the work of Liz Crow best exemplifies, in activism, art, and protest in the United Kingdom, spurred on by the welfare reforms, and the spectacular yet troubling event of the Paralympics, we see new possibilities developed for the relationships between disability and technology.

In these technology-inflected United Kingdom disability protests, we see a creative and effective deployment of digital technology, amounting to a new, potent, and far reaching approach to oppressive and unjust policies, practices, and social relations. Harris, Owen and De Ruiter (2012) have observed that '[a]dvocacy and technology are two core strategies used by the disability community to advance the rights of people with disabilities.'

\section{Conclusion}

In this paper, we have presented two typically distinct, but in our view, tightly and tellingly related perspectives on the topic of disability, activism, and technology.

A threshold issue for people with disabilities, especially, when it comes to such digital technology is barriers to access and use. To explore this complex issue, we discussed disability activism concerning technology. This is well-known in a superficial way when it 
comes to web accessibility, but even here there are very significant dynamics at play. Like other kinds of activism, we might point to the contradiction between commercial, 'closed' platforms-whether Facebook, Twitter, or mobile apps-and the kinds of social, cultural, and political innovation they enable, including activism. There is also a peculiar friction between the still dominant framing of disability technology as 'assistive', versus accounts that argue for technology as part of everyday life for all.

From accessibility and technology, we moved to the notable example of the British protests in which the use of technology, especially social media, was key to strategies and tactics, but also the nature of movements that formed. Preston (2011) has noted the role of Facebook and Twitter in people's political participation and particularly their role in popular uprisings, and questions what role these platforms have played in the protests against disability cuts in the United Kingdom. This is an important question, given that Facebook exceeded more than one billion active users worldwide in 2012. The ubiquitous scale of its adoption makes it an ideal place to curate other elements of social media, and to coordinate and communicate protest activities-as we have suggested in our discussion of British disability welfare protests. Yet in other ways, Twitter plays a crucial role. While Twitter has smaller levels of adoption, its embrace by mainstream media, and what Nicola Bruno (2011) has dubbed the 'Twitter effect' on media coverage, also makes it a powerful platform for activism. Twitter's ability to provide dynamic real time information and organise that information through its hashtags and associated metadata also allow it to act as an alternative broadcast mechanism in its own right.

While these social media platforms played a prominent role in disability activism we discuss in this paper, they do so in conjunction with two other elements. Firstly, Facebook and Twitter in these protests were often part of the now typically cross-referenced (and to some extent commercially integrated) ecology of convergent, online, social, mobile, and locative media technologies and applications-including YouTube, Vimeo, Pinterest, Flickr and Instagram. The widespread availability of mobile digital devices such as smart phones and tablets that allow for the rapid dissemination of these platforms to people both involved in protests but also to others in a timely fashion. Secondly, in the ecologies of social media platforms, there is a mixture of more traditional websites, blogs and email mailing lists. While there have been well documented accessibility problems with much of the hardware and software involved (Ellis \& Kent, 2011), these platforms have also proved to be of great benefit for people with disabilities (Hollier, 2012).

In the United Kingdom welfare protests, then, we see disability activists experimenting with uses of digital technologies, and availing themselves of the 'democratic affordances' they offer (Goggin, 2013). Yet the very fact of the use of digital technologies by people with 
disabilities opens up another contradictory area to do with the technologies themselves and the philosophies and values inscribed in, and affiliated with, them.

The fight for equality in disability and work, and the campaign for justice in welfare, tells us much about the oppressive, unfair, and unsustainable ways that labour and value operate, and work, as a result, is defined in our societies. The parallels here with debates on gender and work are very striking; and, of course, there is an obvious and heavy consequential cross-over between disability and other dynamics of gender, race, class, and sexuality, when it comes to work and welfare. A central part of disability rights and activism is to claim technology as par for the (life) course. Citizenship, participation, and potentiality, occurs now through technological enablement; our acts of activism and daily life are shot through with technology.

So the struggle of disability confronting technology, which has largely passed obscured, misrecognised, and marginalised-via the ritual nod to web accessibility-should finally be acknowledged as going to the core of many of the issues about the philosophies, forms, affordances, architectures, and actions predicated on technology in general. In this paper, we have only made a very preliminary contribution to such a project. In particular, much more research and analysis is needed on the specificities of the particular platforms, and the kinds of communication, social practices, and, potentially, new kinds of politics they support. We are only at the very beginning of recognising that disability justice involves a shake-up and reimagining of democracy itself; the same is true of technology too.

\section{Biographical Note}

Katie Ellis is Senior Research Fellow in the Department of Internet Studies at Curtin University. Her books on disability, media and popular culture include Disability and New Media (2011; with Mike Kent), Disability and the Media (2015; with Gerard Goggin), Disability, Ageing and Obesity: Popular Media Identifications (2014; with Debbie Rodan and Pia Lebeck), and Disability and Popular Culture (2015). Dr Ellis is currently undertaking a DECRA funded project on disability and digital televisions.

Gerard Goggin is Professor of Media and Communications, University of Sydney. He is an Australian Research Council Future Fellow, undertaking a project on disability and digital technology. Gerard's books include Routledge Companion to Disability and Media (2017; 
with Katie Ellis and Beth Haller) and Disability and the Media (2015; with Katie Ellis), and with Christopher Newell, Disability in Australia (2005), and Digital Disability (2003).

Mike Kent is a Senior Lecturer in the Department of Internet Studies at Curtin University. His books on disability, media and education include Disability and New Media (2011) with Katie Ellis and An Education in Facebook (2014) with Tama Leaver.

\section{References}

Ackerman, Spencer. 'Occupy the Skies! Protesters Could Use Spy Drones', Wired 17 November (2011), http://www.wired.com/2011/11/ows-drones/

Adam, Alison and Kreps, David. 'Disability and Discourses of Web Accessibility', Information, Communication \& Society 12.7 (2009): 1041-1058.

Adewunni, Bim. 'Artist-Activist Liz Crow's "Bed-Out”: For Disabled Rights', The Guardian, 10 April (2013), http://www.theguardian.com/artanddesign/2013/apr/09/liz-crow-bed-disabledrights

Andrejevic, Mark. iSpy: Surveillance and Power in the Interactive Era (Lawrence, KS: University Press of Kansas, 2007).

Arnardóttir, Oddný Mjöll and Quinn, Gerard (eds). The UN Convention on the Rights of Persons with Disabilities: European and Scandinavian Perspectives (Leiden: Martinus Nijhoff, 2009).

Atos. 'Welfare Reform', (2015), http://uk.atos.net/en-uk/home/your-business/government/ welfare-reform.html

Barnartt, Sharon N. 'Social Movement Diffusion? The Case of Disability Protests in the US and Canada', Disability Studies Quarterly 28.1 (2008), http://dsq-sds.org/article/view/70/70 BBC. 'Disabled People Hold Nationwide Pretests Against Cuts', BBC News, 22 October (2011), http://www.bbc.com/news/uk-15399724

Blanck, Peter. eQuality: The struggle for web accessibility by persons with cognitive disabilities (Cambridge: Cambridge University Press, 2015).

Blume, Stuart. 'What can the Study of Science and Technology tell us about Disability?' in Carol Thomas, Alan Roulstone, and Nick Watson (eds.), Handbook of Disability Studies (New York: Routledge, 2012), 348-359.

Boler, Megan (ed.). Digital Media and Democracy: Tactics in Hard Times. (Cambridge, Mass: MIT Press, 2008).

Brewer, Judy. 'Access to the World Wide Web: Technical and Policy Perspectives', in Wolf- 
gang Preiser and Elaine Ostroff (eds). Universal Design Handbook (New York: McGraw Hill, 2001), 66.1-66.13.

Bruno, Nicola. 'Tweet First, Verify Later? How Real-Time Information is Changing the Coverage of Worldwide Crisis Events.' Reuters Institute Fellowship Paper, University of Oxford (Oxford, Reuters Institute for the Study of Journalism, 2011).

Bruyère, Susanne and Linda Barrington. Employment and Work (Thousand Oaks, CA: Sage, 2012).

Butler, Patrick. 'How the Spartacus Welfare Cuts Campaign Went Viral', The Guardian, 18 January (2012), http://www.theguardian.com/society/2012/jan/17/disability-spartacus-welfare-cuts-campaign-viral

Butler, Patrick. 'Disability Activists use Social Media to put Care Cuts on the Political Agenda', The Guardian, 21 August (2012), http://www.theguardian.com/society/2012/aug/20/ disability-activists-media-care-cuts

Campbell, S. J. et al. Responsible Reform: A Report on the Proposed Changes to Disability Living Allowance. Diary of a Benefit Scrounger, 9 January (2012), https://onedrive.live.com/ view.aspx?resid=CBA86408918CAA9E!132\&cid=cba86408918caa9e\&app=Word\&authkey=! ACOTRaigEZtaJw8

Campbell, S. J. 'I Support the Spartacus Report', Diary of a Benefit Scrounger, 9 January (2012), http://diaryofabenefitscrounger.blogspot.com.au/2012/01/i-support-spartacus-report. html

Chouinard, Vera. 'Body Politics: Disabled Women's Activism in Canada and Beyond', in Ruth Butler \& Hester Parr (eds). Mind and Body Spaces: Geographies of IIIness, Impairment and Disability (New York: Routledge, 1999), 269-294.

Coronel, Sheila S. 'The Media, The Market and Democracy: The Case of the Philippines', Javnost-The Public 8.2 (2001): 109-126.

Crow, Liz. 'Scroungers and Superhumans: Images of Disability from the Summer of 2012: A Visual Enquiry', Journal of Visual Culture 13.2 (2014): 168-181.

Crow, Liz. 'Bedding Out', September (2013), http://www.roaring-girl.com/work/bedding-out/

Dahlgren, Peter. The Political Web: Media, Participation, and Alternative Democracy (Houndsmill, Basingstoke: Palgrave, 2013).

Dean, Jodi. Democracy and Other Neoliberal Fantasies: Communicative Capitalism and Left Politics (Durham, NC: Duke University Press, 2009).

Eide, Arne H. and Ingstad, Benedicte (eds). Disability and Poverty: A Global Challenge (Bristol: Policy Press, 2011).

Ellcessor, Elizabeth. "<ALT=“Textbooks" $>$ : Web Accessibility Myths as Negotiated Industrial Lore', Critical Studies in Media Communication 31.5 (2014): 448-463. 
Ellcessor, Elizabeth. Enabling Access: Media, Disability, and the Politics of Participation, forthcoming.

Ellis, Katie and Gerard Goggin. 'Disability and Social Media' in Jeremy Hunsinger \& Theresa Senft (eds.) Handbook of Social Media (New York: Routledge, 2014).

Ellis, Katie and Gerard Goggin. Disability and the Media (New York: Palgrave Macmillan, 2015).

Ellis, Katie and Mike Kent. 'Community Accessibility: Tweeters Take Responsibility for an Accessible Web 2.0'. Fast Capitalism 6.2 (2010).

Ellis, Katie and Mike Kent. Disability and New Media (New York: Routledge, 2011).

Erevelles, Nirmala. Disability and Difference in Global Contexts: Enabling a Transformative Body Politic (New York: Palgrave, 2011).

Fleisher, Doris and Zames, Frieda. The Disability Rights Movement: From Charity to Confrontation 2 (Philadelphia, PA: Temple University Press, 2011).

Francis, Leslie and Anita Silver. Americans with Disabilities: Exploring Implications of the Law for Individuals and Institutions (New York and London: Routledge, 2000).

Flynn, Eilionóir. From Rhetoric to Action: Implementing the UN Convention on the Rights of Persons with Disabilities (New York: Cambridge University Press, 2011).

Fuchs, Christian. Occupy Media!: The Occupy Movement and Social Media in Crisis Capitalism (Lanham, MD: John Hunt Publishing, 2014a).

Fuchs, Christian. Social Media: A Critical Introduction (London: Sage, 2014b).

Geere, Duncan. 'Sukey Apps Help Protesters avoid Police Kettles', Wired 31 January (2011), http://www.wired.co.uk/news/archive/2011-01/31/sukey-protest-app

Gentleman, Amelia. 'Disability Protesters go on Remote Offensive', The Guardian, 23 March (2011), http://www.theguardian.com/society/2011/mar/23/disability-protests-benefitcuts-remote-offensive

Goggin, Gerard. 'Democratic Affordances: Politics, Media, and Digital Technology after Wikileaks.' Ethical Space 10.2/3 (2013): 6-14.

Goggin, Gerard. 'Communication Rights and Disability Online: Policy and Technology after the World Summit on the Information Society'. Information, Communication \& Society December (2014) http://dx.doi.org/10.1080/1369118X.2014.989879

Goggin, Gerard and Tim Noonan. 'Blogging Disability: The Interface between New Cultural Movements and Internet Technology', in Axel Bruns and Joanne Jacobs (eds). Uses of Blogs (New York: Peter Lang, 2006), 161-172.

Goggin, Gerard and Christopher Newell. Digital Disability: The Social Construction of Disability in New Media (Lanham, MD: Rowman \& Littlefield, 2003). 
Goggin, Gerard and Christopher Newell. 'Editorial Comment: Disability, Identity, and Interdependence: ICTs and New Social Forms', Information, Communication and Society 9.2 (2006): 309-311.

Grech, Shaun and Karen Soldatic (eds). Disability in the Global South: The Critical Handbook (New York: Springer, 2015).

Haller, Beth. Representing Disability in an Ableist World: Essays on Mass Media (Louisville, KY: Avocado Press, 2010).

Harris, Sarah Parker, Randall Owen and Cindy De Ruiter. 'Civic Engagement and People with Disabilities: The Role of Advocacy and Technology', Journal of Community Engagement and Scholarship 5.1 (2012).

Heymann, Jody, Michael Ashley Stein and Gonzalo Moreno (eds). Disability and Equity at Work (Oxford: Oxford University Press, 2014).

Hickey-Moody, Anna and Denise Wood. 'Virtually Sustainable: Deleuze \& Desiring Differenciation in Second Life', Continuum 22 (2008): 805-816.

Hollier, Scott. Sociability: Social Media for People with a Disability (Sydney: Media Access, 2012), https://accan.org.au/our-work/research/444-sociability-social-media-for-people-witha-disability

Hughes, Bill. 'Disability Activisms: Social Model Stalwarts and Biological Citizens', Disability \& Society 24.6 (2009): 677-688.

Imrie, Rob and Rachel Luck. 'Designing Inclusive Environments: Rehabilitating the Body and the Relevance of Universal Design', Disability and Rehabilitation 36.16**(2014): 1315-1319.

Ismail, Sarah. 'Why No Mention of Kettling Disabled Protesters', The Guardian, 31 January (2011), http://www.theguardian.com/society/joepublic/2011/jan/31/kettling-disabled-protesters-welfare-reform

Kelly, Christine. 'Towards Renewed Descriptions of Canadian Disability Movements: Disability Activism outside of the Non-Profit Sector', Canadian Journal of Disability Studies 2.1 (2013), http://dx.doi.org/10.15353/cjds.v2i1.68

Krieger, Linda Hamilton. Backlash Against the ADA: Reinterpreting Disability Rights (Ann Arbor, MI: University of Michigan, 2003).

Kulick, Don and Jens Rydström. Loneliness and Its Opposite: Sex, Disability, and the Ethics of Engagement (Durham, NC: Duke University Press, 2015).

Lewthwaite, Sarah. 'Web Accessibility Standards and Disability: Developing Critical Perspectives on Accessibility.' Disability and Rehabilitation 36.16 (2014): 1375-1383.

Lievrouw, Leah A. Alternative and Activist New Media. (Cambridge: Polity, 2011).

Lindsay, Colin and Donald Houston (eds). Disability Benefits, Welfare Reform and Employment Policy (New York: Palgrave Macmillan, 2013). 
Macrae, Ian. 'Why Liz Crow is Bedding Out', Disability Now May (2013), http://www.disabilitynow.org.uk/article/why-liz-crow-bedding-out

Marin, Bernd, Christopher Prinz and Queisser, Monika (eds). Transforming Disability Welfare Policies: Towards Work and Equal Opportunities (Aldershot, UK: Ashgate, 2004).

McArdle, John. 'UK Welfare Reform Deaths-Updated List'. Black Triangle Campaign, 21 October (2014), http://blacktrianglecampaign.org/2014/10/21/uk-welfare-reform-deaths-updated-list-october-21st-2014/

Meikle, Graham. Future Active: Media Activism and the Internet (Sydney: Pluto Press; London: Routledge, 2012).

Mills, Mara. 'Deafening: Noise and the Engineering of Communication in the Telephone System', Grey Room 43 (2011): 118-143.

Morse, Felicity. 'Scuffles At Atos Paralympics Protest As Police and Disability Campaigners Clash', The Huffington Post (UK) 31 August (2012), * http://www.huffingtonpost. co.uk/2012/08/31/atos-paralympics-protest-uk-uncut-disability-_n_1846633.html

Morton, Rick and Rachel Baxendale. 'ABC Kicks Out Website Protest', The Australian, 1 July (2014), http://www.theaustralian.com.au/media/abc-kicks-out-website-protest/storye6frg996-1226972886005

Moser, Ingunn. 'On Becoming Disabled and Articulating Alternatives: The Multiple Modes of Ordering Disability and their Interferences', Cultural Studies 19 (2005): 667-700.

McRuer, Robert. Crip Theory: Cultural Signs of Queerness and Disability (New York: New York University Press, 2006).

Newell, Christopher. 'The Disability Rights Movement in Australia: A Note from the Trenches’, Disability \& Society 11.3 (2006): 429-432.

Oliver, Mike, and Colin Barnes. The New Politics of Disablement. (Houndsmills, UK: Palgrave, 2012).

Padovani, Claudia and Andrew Calabrese (eds). Communication Rights and Social Justice: Historical Accounts of Transnational Mobilizations (Basingstoke, UK: Palgrave Macmillan, 2014).

Peck, Sunil. 'Direct action! Life on the streets', Disability Now, November (2010), http:// www.disabilitynow.org.uk/article/direct-action-life-streets

Pelka, Fred. What Have We Done: An Oral History of the Disability Rights Movement. (Amherst, MA: University of Massachusetts Press, 2012).

Pertierra, Raul, Eduardo F. Ugarte, Alicia Pingol, Joel Hernandez, and Nikos L.Dacanay. Txt-ing Selves: Cellphones and Philippine Modernity. (Manila: De La Salle University Press, 2002).

Preiser, Wolfgang F. E. and Elaine Ostroff (eds). Universal Design Handbook. (New York: McGraw-Hill, 2001). 
Preston, Claire. 'Resisting Disability Benefits Cuts in a Digital Age', paper presented at the Social Policy Association Conference. University of Lincoln, 4-6 July (2011), http://www. social-policy.org.uk/lincoln2011/Preston\%20P6.pdf

Ratto, Matt, and Megan Boler (eds). DIY Citizenship: Critical Making and New Media. (Cambridge, MA: MIT Press, 2014).

Roulstone, Alan. Enabling Technology: Disabled People, Work, and New Technology (Buckingham, UK, and Philadelphia, PA: Open University Press, 1998).

Roulstone, Alan. Understanding Disability Policy (Bristol: Policy Press, 2012).

Ryan, Frances. 'Social Media Means the Voices of the Disabled Can no Longer be Ignored by Those in Power, New Statesman, 7 August (2014), http://www.newstatesman. com/2014/07/social-media-means-voices-disabled-can-no-longer-be-ignored-those-power

Samuels, Ellen. Fantasies of Identification: Disability, Gender, Race. (New York: New York University Press, 2014).

Sandell, Richard, Jocelyn Dodd and Rosemarie Garland-Thomson (eds). Re-Presenting Disability: Activism and Agency in the Museum (London and New York: Routledge, 2010).

Schuler, Douglas and Aki Namioka (eds). Participatory Design: Principles and Practices (Hillsdale, NJ: Erlbaum Associates, 1993).

Scotch, Richard. 'Disability as the Basis for a Social Movement: Advocacy and the Politics of Definition', Journal of Social Issues 44.1 (1988): 159 - 72.

Shakespeare, Tom. 'Disabled People's Self-Organisation: A New Social Movement?', Disability, Handicap and Society, 8.3 (1993): 249-264.

Shakespeare, Tom, ed. Disability Research Today: International Perspectives (London and New York: Routledge, 2015).

Shapiro, Joseph. No Pity: People with Disabilities forging a New Civil Rights Movement (New York: New York Times Books, 1993).

Shildrick, Magrit. Dangerous Discourses of Disability, Subjectivity, and Sexuality. (Basingstoke, UK: Macmillan, 2009).

Snyder, Sharon, and David T. Mitchell 'Re-engaging the Body: Disability Studies and the Resistance to Embodiment', Public Culture, 13.3 (2001): 367-389.*

Soldatic, Karen and Helen Meekosha. The Global Politics of Impairment and Disability: Processes and Embodiments (London: Routledge, 2014).

Soldatic, Karen, Hannah Morgan and Alan Roulstone (eds). Disability, Spaces and Places of Policy Exclusion (London and New York: Routledge, 2014).

Soldatic, Karen, Kim Spurway and Helen Meekosha. 'Hard Yakka': Living with a Disability in the West Kimberley (Sydney: University of New South Wales, 2014), http://www.daa.wa.gov. 
au/Documents/ReportsPublications/Staff\%20Documents/Whats\%20on\%20Today/Hard_Yakka_July_2014_Soldatic-Spurway-Meekosha-libre.pdf

Sourbati, Maria. 'Disabling Communications? A Capabilities Perspective on Media Access, Social Inclusion, and Communication Policy', Media, Culture \& Society 34.5 (2012): 571-587.

Taylor, Matthew. 'Students Protests Video allegedly shows Police Pulling Man out of Wheelchair', The Guardian, 14 December (2010), http://www.theguardian.com/uk/2010/dec/14/ student-protests-video-protester-wheelchair

Thomas, Nicholas. Entangled Objects: Exchange, Material Culture, and Colonialism in the Pacific (Cambridge, MA: Harvard University Press, 1991).

Titchkovsky, Tanya. The Question of Access: Disability, Space, Meaning (Toronto: University of Toronto Press, 2011).

Tracey, Emma. 'Artist Goes to Bed in Public to Raise Disability Awareness', (2013), http:// www.bbc.co.uk/blogs/legacy/ouch/2013/03/artist_goes_to_bed_in_public_t.html Trevisan, Filippo. 'Disabled People, Digital Campaigns, and Contentious Politics: Upload Successful or Connection Failed?', in Richard Scullion, Roman Gerodimos, Danile Jackson and Darren Lilleker (eds). The Media, Political Participation, and Empowerment (London: Routledge, 2013), 175-191.

Trevisan, Filippo. Disability Rights Advocacy and New Media in Britain and America (London: Routledge, 2015).

Trevisan, Filippo. 'Scottish Disability Organizations and New Media: A Path to Empowerment or 'Business as Usual'?', Disability Studies Quarterly 34.3 (2014), http://dsq-sds.org/ article/view/3359/3648

Tsing, Anna Lowenhaupt. Friction: An Ethnography of Global Connection (Princeton, NJ: Princeton University Press, 2005).

Twitter. 'Improving accessibility of Twitter.com' December 2014 (2013) from https://blog. Twitter.com/2013/improving-accessibility-of-Twittercom

Twitter. 'Twittera11y team', (2014) https://Twitter.com/a11yteam

Waddington, Lisa, Gerard Quinn and Eilionóir Flynn (eds). European Yearbook of Disability Law 5 (Cambridge, UK: Intersentia, 2015).

White, Peter. 'The Triumph of Hope over Reality', (2013), http://disabilitynow.org.uk/article/ triumph-hope-over-reality

Youmans, William Lafi and Jillian C. York 'Social Media and the Activist Toolkit: User Agreements, Corporate Interests, and the Information Infrastructure of Modern Social Movements', Journal of Communication, 62.2 (2012): 315-329. 
The LOCKSS System has the permission to collect, preserve and serve this open access Archival Unit

This Isuue of the Fibreculture Journal by The Fibreculture Journal Incorporated is licensed under a Creative Commons Attribution 4.0 International License.

\section{OPEN HUMANITIES PRESS}

The Fibreculture Journal is published by The Fibreculture Journal Incorporated in partnership with Open Humanities Press. 
issue 26: Entanglements - Activish and Jechnology

\title{
FCJ-189 Reimagining Work: Entanglements and Frictions around Future of Work Narratives
}

\author{
Laura Forlano \\ Illinois Institute of Technology
}

Megan Halpern

Arizona State University

\begin{abstract}
:
This article explores entanglements and frictions between labour advocates and their relationship to technologies in future of work narratives in the United States. Drawing on literature from science and technology studies and media studies, we argue that there is an opportunity to move beyond discussions of technology as a 'black box' in order to deepen these entanglements and expose the frictions within these discourses. As a means of engaging with the ways in which the future of work is being imagined and opening up discussions around technology, we conducted a one-day participatory design workshop that uses a game in order to reveal the philosophies embedded in labour activism and technology.
\end{abstract}

doi: $10.15307 / f c j .26 .189 .2015$ 


\section{Introduction}

This paper discusses the ways in which labour advocates are enmeshed and entangled in narratives around the role of emerging technologies such as automation, artificial intelligence, and robotics in the future of work. The article draws on literature from science and technology studies and media studies in order to critically analyse narratives about the future of work in the mainstream media. Based on interviews and a design workshop, we argue that participatory design methodologies are one way to engage with and explore the frictions inherent in these future of work narratives in order to find productive ways of bridging the philosophies embedded within labour activism and technology.

Technologies such as crowdsourcing platforms, 'just in time' scheduling software, big data tracking, and robots are at the forefront of discussions around the future of work in the mainstream media. To a large extent, these powerful and far reaching narratives focus on the replacement of workers with technologies in a linear and technologically deterministic manner rather than acknowledging the complex, non-linear, and iterative relationship between activism, social change, and technology. While labour activists continue to advocate for changes that might benefit workers, they are often reacting to the ways in which these debates are framed around emergent technologies. The gaps between the philosophies embedded in labour activism and these technologies are distant. Furthermore, there is little engagement between labour activists and technology activists, who might share some of their values but are positioned differently with respect to their engagement with technology. For example, while labour advocacy groups are canvassing and campaigning to restore and improve the rights of workers, progressive technology activists are advocating for openness, cooperation, and transparency around issues such as network neutrality, intellectual property, and the digital divide.

In order to examine these entanglements and frictions, in July and August 2014, we conducted a three-month project on the future of work. The project included one-hour interviews with 14 labour advocates in Chicago as well as the design of a half-day participatory design workshop. The labour advocates that we interviewed included representatives from organisations working on behalf of youth, immigrants, women, African Americans, Latinos and formerly incarcerated people with a specific focus on the lowest paid and least protected workers including restaurant workers, domestic workers, and healthcare workers. Our interviews revealed that labour activists are both reacting to narratives around the future of work that make revolutionary claims about technological replacement and, on the other hand, engaging with technology such as the Internet and social media in order to mobilise workers around important issues. Thus, somewhat counter intuitively, our interviews demonstrate that, for labour activists 
technology is in some ways still an impenetrable 'black box' while at the same time a site where socio-political values can be enacted for the benefit of activism. Put in other terms, the revolutionary, disruptive, fearful, and dystopian claims of technologists and, somewhat in complicity, economists around technological displacement coexist with more nuanced evolutionary understandings of the ways in which specific tools-in this case, communication technologies-can be developed from a position of greater agency.

The purpose of the workshop was to use design methods in order to explore current and historical relationships between labour and technology as a means of opening up a dialogue on the possibility of imagining and prototyping technologies that could embed philosophies from labour activism. Specifically, we argue that there is an opportunity to identify productive frictions that might allow for the creation of technologies designed around alternative value systems that protect fair and just working conditions. By prototyping such technologies, it is possible to create experiments and demonstrations of how these alternative values might serve to protect and advance the rights of workers. These prototypes could then function as examples that could intervene in future of work narratives in ways that more clearly and accurately represent the philosophies of labour advocates. In addition, a deeper engagement between the philosophies of labour advocacy and technology might help to build technological literacy among labour advocates and social justice literacy among technology activists. This shared literacy could serve to counter claims around technological replacement and, instead, emphasise the need for a reconfiguration of work in collaboration with technologies that are developed, appropriated, and used to create the conditions for a more socially and economically just future. This future could be achieved through the design of our technological systems in tandem with the deliberate effort to shape public policies that reduce inequality, resist discrimination, increase living standards and allow for people to be treated with dignity and respect.

\section{Methodology}

In order to understand the entanglements and frictions between the philosophies embedded in activism and technology, we used several methodologies, including: the analysis of over $\mathbf{2 7 5}$ mainstream media articles; fourteen semi-structured, hour-long qualitative interviews; and, the creation of a participatory design workshop. We conducted interviews with activists from organisations that served diverse communities of workers, ranging from telecommunications workers to domestic workers. While the majority of the activists and organisers were based in Chicago; two were based elsewhere. Eleven of these interviews were conducted prior to the workshop in order to inform its design and three were conducted after the workshop in order to evaluate its results. 
These interviews focused on three concepts related to the work organisers were doing in their respective organisations, and the attitudes they thought they were seeing from their organisations and constituents. The first line of questioning focused on their perceptions of the current and future state of labour practices in the United States, and included questions about challenges and opportunities facing the labour force and their organisations. For the second theme, which was technology, interviewees were asked about their own relationship to technology, the impact they felt it had on their constituents, and work they were to doing to manage that impact. Finally, interviewees were asked about how their organisations planned for the future, and how they and their constituents talked about the future. The purpose of these interviews was to gain a better understanding of pressing issues related to the role of technology in the future of work as well as the kinds of philosophies and values that shaped the work of labour activists in order to inform the design of a workshop. Another reason for the interviews was to reach out to labour activists in order to build relationships with individuals that might be interested in participating in the workshop as well as to get recommendations of additional labour activists and organisations that might want to be interviewed or participate in the workshop. This outreach and interview phase was important to ensure that we had a wide range of groups represented including those that worked on behalf of women, immigrants, youth, formerly incarcerated people, African-Americans, and Latinos in the Chicago area.

Building on the initial interviews and drawing on literature about material deliberation, participatory design, and speculative design, we designed a half-day workshop about technology and the future of work. Public engagement with science and technology often bemoans the lack of what is referred to as upstream engagement in discussions regarding science and technology policy (Hilgartner, 1990). Efforts to create space for voices from publics and their representatives, like citizen juries and consensus conferences, are often esoteric and tedious, and their outcome is often dubious (Joss, 1999; Wynne 2006). While there is a growing movement to develop more approachable methods of deliberation for voicing public concerns about the uses of emergent technologies, it is difficult to gain public interest, or to break through existing narratives. Efforts in anticipatory governance (Barben et al., 2008) aim to anticipate the long-term implications of emerging technologies through methods such as forecasting, public deliberation, scenarios, foresight, and vision assessment. With respect to alternative ways of thinking about deliberation in science and technology studies, Davies et al. (2012) discuss the need for exploring forms of material deliberation. 'It is important to make use of different formats and approaches which highlight not (only) reasoned arguments for particular developments but also the affective connections, materialities, and experiences which structure public interactions...' (2012: 356). While their focus was on the new city, the same principle can be broadly applied to the future of work. In this spirit, we engaged with several design practices and methodologies to develop a kind of material deliberation for labour activists. 
To create an interaction that would provide opportunities for material deliberation, we turned to design methodologies including participatory design and speculative design. We selected participatory design specifically because it was developed in the 1970s and 1980 s in Scandinavia as a means of 'empowering workers and fostering democracy in the workplace' with respect to the development of technology systems through partnerships (Spinuzzi, 2005: 2). According to Spinuzzi:

This avowedly political research aimed to form partnerships with labour unions that would allow workers to determine the shape and scope of new technologies introduced into the workplace. Up to that point, labor unions had little experience with computer technologies and had been forced to accept systems developed by management, systems that represented a sharp break from workers' traditional ways of working; exerted a greater and greater control over increasingly fine details of their work; and automated large swathes of the workflow, putting people out of work (see Ehn, 1990; Zuboff, 1989).

Rather than merely accepting or rejecting technologies that were adopted into the workplace, researchers hoped to provide ways for software developers and workers to develop technologies together, thereby allowing for greater control by workers. Through participatory design, diverse stakeholders can share their ideas, become exposed to the ideas of others, and generate new ideas. Recent design scholarship explores the link between design-whether through objects, exhibits or workshops-and the construction of publics and building of political constituencies and publics around important policy issues (DiSalvo, 2009). Tensions and frictions are particularly relevant to the work of participatory design because according to adversarial design (DiSalvo, 2012), there are many potential benefits to political conflict. Finally, participatory design can serve to raise conceptual questions and form constituencies and publics around important 'matters of concern' (DiSalvo, 2009; DiSalvo, Lodato, Fries, Schechter, and Barnwell, 2011; Latour \& Weibel, 2005).

Few disciplines are equipped to engage with future conditions and concerns in order to raise important questions about alternative possibilities and 'what if' scenarios. Rather than relying on the more deterministic methodology of future forecasting, we turned to speculative design, which emphasises the possibility of multiple alternative futures and uncertainties. Speculative design is also relevant to material deliberation, especially when considering technologies that are not fully developed, or about which there is much concern but little experience. Speculation can give voice to fears and concerns, or it can be a tool for change. Speculative design can create ways of harnessing the drive to think about 'what might be' to help shape 'what will be.' Thus, we drew on speculative 
design methodologies because they allow for a more generative, speculative and futureoriented space of alternative possibilities (Bleecker, 2009; DiSalvo, 2012b; Dunne, 2001). Speculative design allows for issues and ideas to transcend the temporal constraints of the here and now. By moving these methods spatially outside of museums and galleries and into participatory design workshop settings, we are able to use them to transcend both temporal and spatial boundaries.

Approximately twenty-five people including labour activists (8), technology activists (2), designers (9), funders (3) and scholars (3) attended the workshop. Four of the workshop participants were interviewed as part of the project-two before the workshop and two after the workshop. Workshop participants were not paid. One of the authors participated directly in the workshop while the other primarily facilitated and observed. The workshop had two parts. The first part enlisted participants in a board game designed by the authors using critical game theory (Flanagan and Nissenbaum, 2014) and reflective design practices like cultural probes (Gaver, Dunne and Pacenti, 1999). The game was designed for up to four teams of two to four players. Two games were played in tandem with one of the authors participating and observing each game, while a graduate student observed and reported to the authors her impressions of game play. The games each had twelve players total, with three players on each team. Laura Forlano participated on a team, while Megan Halpern observed and answered questions (she was the thirteenth player at the table). The game was an exercise in thinking speculatively about both the past and the future and it represented a timeline that spanned five eras, beginning with ancient Greece and Egypt, and ending in the year 2050. Each space on the board was within an era, and featured a specific point of view. For example, a team might land in "Era 2", on a space labeled "Child factory worker" and might draw a card that asks them to create a list of demands. Or later in the game, they might land on a space that identifies them as a robot worker, and tasks them with sending a postcard from their vacation.

The game allowed workshop participants to explore historical and present technologies, socio-economic conditions, and labour realities in order to open up discussions around the way in which technologies shaped and were shaped by social, economic, political and cultural contexts. It also allowed labour activists to collaborate with scholars, designers and technologists around the creation of counterfactual histories that might allow for alternate relationships, outcomes and possibilities that might benefit workers. However, we were specifically interested in how this engagement might allow for the design of future technologies that might embed the philosophies of labour activists. As a result, the second part of the workshop tasked groups to work together to design an object, prototype, experiment, or platform that drew on the most unexpected or counterintuitive moments during the game play. In order to do this, participants reviewed the ideas that they had come up with as part of imagining counterfactual histories as well as how these ideas 
expressed their values and used them as a basis for the creation of the prototypes. Groups were asked to specify who they were designing for including demographic characteristics such as race, class, gender, economic status, and industry.

Each team from Game A joined with a team from Game B to complete the prototype. Each of the four prototype teams had six members, and each team had forty-five minutes to create their prototype using materials that were in the room. We provided low-fidelity supplies such as cardboard boxes, blue tape, markers, and twine, to encourage the groups to create objects that more closely resembled sketches than polished prototypes. To better understand the impact of the workshop, we paused between activities and at the end of the workshop to ask questions and invite reflections on participants' experiences. These brief focus groups were recorded and analysed along with the interviews that guided the development of the workshop.

\section{Death by Robot}

Mainstream media accounts are full of powerful, revolutionary narratives around the role of emerging technology and the future of work, which position automation, artificial intelligence and robots in opposition to humans in the workforce. The claim is thatfuelled by investment, research and development, Moore's Law (the observation that the number of transistors on integrated circuits doubles every two years), sensors, and the Internet-technologies will replace and/or make many jobs redundant ("Rise of the robots", 2014. According to the proponents of this view, which include many economists and technologists, robots, driverless cars, and drones will 'drive down the value of human labour with astonishing speed' and create 'hordes of citizens of zero economic value' (Davidow and Malone, 2014).

If the first machine age-driven by steam power-resulted in an increase in wages and the standard of living, the 'second machine age' (Brynjolfsson and McAfee, 2014)-driven by big data, computing, and processing-will result in a decrease in wages and the standard of living. These applications of technologies are primarily in three areas: interacting with the physical world, language, and problem solving. One recent book argues that:

the corporate sector is relying on information technology both to simplify and accelerate the processes of business output, and so increase the output of labor, and to deskill labor, 
diminish its role, and so weaken its earning power. The widening gap between the growth of labor's output and its real earnings is the desired outcome of this regime (Head, 2014).

According to a recent study by Frey and Osborne (2013), 47\% of jobs in the United States are believed to be 'at risk' of computerisation. On the other hand, while it is highly likely that the majority of jobs will be reconfigured in some way due to automation and robotics, MIT's David Autor believes that accounts of technological displacement are widely overstated. Currently, it is still difficult for machines to perform tasks that require adaptability, common sense, or creativity (Autor, 2014).

For example, according to many accounts, between 34\% [1] and 44\% [2] of the current active workforce in the United States are contingent workers (a 70\% increase since 2008 according to temporary placement firm Kelly Services), which might include job categories such as freelance, temporary, interns, part-time, self-employed, project based, consultants, contract, and independent workers. Over $40 \%$ of people are currently working or have worked as independent workers. [3] As of 2006, the General Accounting Office estimated that one third of the US workforce or 42 million workers work independently (self-employed, independent contractors, temps, part timers, etc.). Similarly, another study by the Association for Enterprise Opportunity claims that $92 \%$ of all US businesses could be characterized as microbusinesses in that they have fewer than 5 employees, a total of 41.3 million jobs. [4] In February 2014, the Bureau of Labor Statistics reported that 14.4 million Americans were self-employed in areas such as agriculture, which are shrinking, and others such as services, which are growing. Finally, by 2020 , it is believed that roughly $50 \%$ of the private workforce will have spent time as independent workers at some point in their work lives. [5]

In addition, platforms for managing and coordinating contingent work have given rise to new forms of control over workers as well as new job roles. There has been widespread media coverage of so-called sharing economy platforms such as Uber (a taxi service) and Airbnb (an accommodation rental service). However, at the same time, less known forms of work have emerged. These include: TaskRabbits (people that do small tasks and errands such as cleaning and household chores, fixing things and helping with packing and moving as coordinated by online, mobile marketplaces, one of which is called TaskRabbit), Turkers (people that perform work through digital labour platforms such as Amazon Mechanical Turk), cam girls (people that perform live online pornography and sex shows) and gold farmers (people playing video games in order to earn virtual currency, which is exchanged for real money). The Internet and other platforms have also created emergent forms of free and unpaid labour; such as, the work that you do when you check out for yourself when shopping online as well as increasingly at the grocery store, pharmacy, or Apple store. 
According to a recent report on the Future of Artificial Intelligence, Robotics, and the Future of Jobs conducted by the Pew Research Center in August 2014 based on responses from 1,896 experts, it is likely 'robotics and artificial intelligence will permeate wide segments of daily life by 2025 , with huge implications for a range of industries such as healthcare, transport and logistics, customer service, and home maintenance' (2014: 1). The report identifies a number of utopian and dystopian narratives that run through the expert perspectives. Specifically, experts are hopeful that technology will be a net creator of jobs, new types of work will be created, new relationships with work will be more positive, and we will be able to better control our own destiny. On the other hand, experts are concerned that automation will disrupt white-collar work, that many people will become unemployed or underemployed in lower wage jobs, and that our educational system is failing to prepare us for the future.

While emerging technologies will likely disrupt and reconfigure jobs, employment and the future of work, they are unlikely to replace humans altogether. At the same time, these displacements may alter the quantity and quality of work as well as the type of work we will do in the future with the creation of completely new job categories. Futhermore these displacements may be felt more severely in some sectors, jobs and populations than in others. So, why have narratives around the replacement of humans by robots come to dominate the mainstream media coverage around technology and the future of work? Who is framing the debate in this way and who is benefitting from this particular framing? How are the views of labour advocates being included or excluded from these narratives? Where are there entanglements and frictions between technologies, as they are being represented, and the philosophies of labour advocacy? How might design be a way to open up these debates and reveal possibilities for productive engagement between activism and technology? Accounts by labour advocates as well as scholarly understandings around the role of technology and the transformation of work may allow us to better understand these entanglements and frictions. For example, recent scholarship has also considered the role of digital labour (Scholz, 2012), free labour (Terranova, 2000), unpaid labour (Andrejevic, 2012), immaterial labour (Lazzarato, 1996), and affective labour (Hardt, 1999).

\section{Frictions and Entanglements between Technologies and Labour Activists}

This paper seeks to understand-through a lens of science and technology studies and media studies - the entanglements that arise due to frictions between the philosophies embedded within technologies and the philosophies embedded within labour activism as 
evidenced by the discourses and narratives around the future of work. According to the Oxford Dictionaries, there are two distinct meanings of the term friction both of which are related to the discussion about activism and technology. First, "the resistance that one surface or object encounters when moving over another'; and, second 'conflict or animosity caused by a clash of wills, temperaments, or opinions.' [6] In particular, with respect to the role of technology and its relationship to work and labour activism, the references to both resistance and conflict are relevant.

In particular, we discuss the ways in which labour activists might engage more deeply with technologies, necessarily requiring the negotiation of acceptance, compromise, or submission (Tsing, 2005). According to Tsing, 'Cultural diversity brings a creative friction to global connections...These zones of cultural friction are transient; they arise out of encounters and interactions...[they are] the awkward, unstable and creative qualities of interconnection across difference' (2005: 4). Tsing proposes that friction is a metaphor that captures the diverse and conflicting zones of awkward engagement that make up our contemporary world. We might also consider these frictions with respect to notions of dissensus (Mouffe, 2003), conflicts (Hillgren, Seravalli, and Emilson, 2011) and agonism (DiSalvo, 2012). We believe that this friction is both disruptive for labour advocates as well as productive for the development of technologies framed around alternative future possibilities, which embed notions of fairness, justice, and equality. By examining these frictions with respect to narratives around the future of work, it is possible to critically analyse engagements between labour advocates and technologies.

Mainstream media accounts emphasise a certain revolutionary and technological determinism that is nameless, faceless, and without context. The future is represented as either a utopia of leisure in which humans no longer need to work or a dystopia of competition in which humans must compete with machines in order to survive. In this framing, technology is a 'black box'. It is a disembodied, objective and neutral tool that is used within a capitalist system to control, manage and replace workers. As such, there is little that humans, let alone labour advocates, can do to prevent its adoption and use. With this framing, it is only natural that labour advocates voice fears about technological unemployment, job scarcity and declining growth rates, and the implications for the middle class and wages in the United States.

Yet, in some ways, for labour organisers technology is simultaneously a 'black box,' which is impenetrable, and a tool for change and activism. For example, Edward, who ran a group that advocated for day labourers and domestic workers, did not have concerns about automation, whereas Alan, who worked in telecommunications, had already seen many jobs lost in the wake of simplified communications technology, but saw technology as a 
tool for fulfilling the need to retrain workers to help them rejoin the workforce. This dual nature of philosophies embedded within technology seems to further complicate existing social theories, which consider technology either as a 'black box' with complete agency over the social or as a social construction that coevolves with the social. However, more recent scholarship around socio-materiality (Orlikowski, 2007), digital materiality (Dourish and Mazmanian, 2011; Leonardi, 2010; Robles and Wiberg, 2011), object-oriented ontology (Bogost, 2012) and new materialism (Barad, 2003; Parikka, 2012) has attempted to account for this agency of artifacts, objects and technologies while at the same time explaining their socially constructed nature in what is becoming understood as a turn back towards materialism.

This approach to materialism can be observed not only in mainstream media accounts around technology but it is also present in our interviews with labour advocates. For example, according to one union organiser:

DARREN: I don't think that there is an example in human history of technology being rejected because of the disruption that it creates, ... At the end of the day, technology wins in military history, in labor history. ... Is Moore's Law an equivalent of the transition from steam to electricity or is it a permanent thing? You get completely different alternative futures depending on what you believe. ... If you believe that Moore's Law will settle down then you will have labor shortages, declining birthrates, not enough people for the work that needs to be done and fewer younger people than there are old people. ... If Moore's Law continues, then you have technological unemployment for the first time in human history ... not short-term, not disruptions that produce migrations and resettlements to different sectors or different geographies. ... How do you solve for human prosperity when there is a scarcity of work for the first time? There are the two very different futures. In one, there are 7 billion people competing for 2 billion jobs. ... There are signs of both futures out there.

In contrast to this view, an alternative framing of the philosophies embedded within technologies might be one of evolutionary change in which technologies are socially constructed and mutually constituted (Bijker and Law, 1994; Pinch and Bijker, 1984), embedded in socio-economic and socio-technical systems (Star, 1999), reconfigurations of invisible labour (Latour, 1992) and imbued with society's values (Friedman and Nissenbaum, 1996; Nissenbaum, 2001). For example, in order to provide an alternative framing of technology, it is important to challenge the notion that Moore's Law is a scientific law. Rather, it is an observation that became embedded into corporate culture as an engineering goal or metric for success in order to sustain sales and profits (Dourish and 
Bell, 2011). This helps to open up a discussion around the ways in which particular values and even temporalities become embedded into socio-technological systems that shape work and the economy.

Suchman has discussed the ways in which Silicon Valley projects, constructs and claims certain kinds of futures, while, at the same time, deliberately excluding and ignoring other futures, people and geographies (2011). While 18-month cycles of planned obsolescence (Fitzpatrick, 2011; McDonough and Braungart, 2002) shape the revolutionary discourses around which corporate technologies are developed, marketed and sold, the temporalities and futures as experienced by labour advocates are quite different. Rather than taking the lead to frame issues and discourses about the future of work, labour advocates are often responding to the actions of business and government. Especially when it comes to discussions around technological change, labour advocates acknowledge that they are often reactionary in nature since they have sometimes failed to understand the kinds of technological changes that will likely transform the future of work.

For the most part, the work of labour advocates (as well as other forms of activism) is demarcated in relatively short increments, from 3-6 months to campaign cycles to annual funding deadlines. While different organisations confront different timelines, the everyday pressures on their plans and schedules, make it difficult to think beyond immediate goals and constraints. At the most expansive, 'the future' was characterised as a 5-year strategic plan. For example, one immigrant rights advocate, Jonathan, characterised three specific time horizons in which his work was divided: near term (current policy decisions being made by the administration), long term (within the next 5-years and into the second term of a new President) and very long range (large structural changes that have been continuing since the 1970s). However, according to David, an advocate for healthcare workers, while there are immediate and longer-term issues that shape the nature of the work, overall, time is a continual process that is changing and fluid in relation to developments in healthcare. This conflict between the philosophies of time that are embedded in technologies and those embedded in labour activism offers one site of potential engagement, which we will take up in the next section of the paper. In fact, the very notion of chronological time (and, therefore, ideas about the future) is currently being challenged by recent discussions in queer feminist scholarship (Barad, 2007).

With respect to the entanglements between human and non-human actors and the delegation of competencies (Latour, 1992), in a recent book review, Irani points out that the overemphasis on artificial intelligence and robotics misses two important points: first, that these technologies are actually made possible by huge amounts of invisible labour and, second, that 'Automation doesn't replace labour. It displaces it,' (2015: 2). Yet, according 
to Joseph Thomas Phelan, a communications strategist within the emergent worker justice movement:

the 'the robots are coming' argument has some very amazing and compelling stories that tie neatly into popular culture narratives about both the promise and peril of the rise of the robots. As an organiser l've learned that it is these small stories and meta-narratives wired into emotions and values that drive people's actions, regrettably not facts. [7]

Furthermore, these narratives about technology reify, and even degrade, existing social and economic realities rather than signalling opportunities to shift priorities and values. Technology has affordances (Gibson, 1977; Norman, 1990) and limitations that interact with and shape social systems; however, the ways in which we design, appropriate, and use technologies occurs in dialogue with socio-economic systems. As Zeynep Tufekci argues in light of narratives around the need for caregiver robots that will mind our children and accompany us through old age, 'This is not just an inhuman policy perspective, it's economically destructive and rests on accepting current economic policies and realities as if they were immutable' (2014: 1). Domestic workers and home care workers are some of the lowest paid and most poorly treated contingent workers in the United States (Tufekci, 2014) because they have few legal protections. Tufekci argues that this kind of emotional labour which is to a large extent performed by women and immigrants, in particular, is undervalued and therefore under paid and (sometimes) unpaid.

For the labour advocates we interviewed emerging technologies are both understood as a 'black box' and, at the same time as tools, for activism and campaigning. One the one hand, technologies such as artificial intelligence and robots are too abstract and, as a result, it is not surprising that labour advocates adopt a more fearful perspective when compared to their attitudes towards other kinds of technologies. At the same time, communication technology such as social media and the Internet were valued as having great potential for labour advocacy groups. Some groups do not think about technology very much, and some not at all, within the context of their work.

There are several specific entanglements and frictions between the philosophies embedded within labour activism and the philosophies embedded within technology that we have identified: participation and control, skills and literacy, and access and appropriation. With respect to discourses around technology and media related to participation and control, in recent years, there has been considerable scholarship on topics including civic engagement (DiSalvo, 2009; Foth, Forlano, Gibbs, and Satchell, 
2011; Gordon and Koo, 2008), participatory culture and participatory media (Bruns and Burgess, 2011; Burgess, Foth, and Klaebe, 2006; Burgess and Green, 2013; Rheingold, 2008) and open source software and peer production (Benkler, 2006). One way in which these discussions play out around future of work narratives is with respect to 'just in time' scheduling technologies. For example, according to a position paper by Gleason and Lambert:

New protections and greater voice for workers are needed to realize the potential that workforce technologies have for restoring a fair workweek for millions of hourly workers. When technology is something solely controlled by management to increase profit, in a context where workers are almost all unorganised, a future of work where most workers are pushed even further to the margins of society is all but guaranteed (2014: 5).

Since the adoption and use of these technologies are controlled by management rather than by workers-and/or through negotiation between management and workers-these technologies are imbued with values associated with increasing profit by efficiently allocating work hours. In this case, there is friction between the values embedded in 'just in time' scheduling platforms and the values that labour activists are working towards to guarantee a fair workweek. These two sets of values need not necessarily be in conflict but they reflect deeper inequalities in the economy with respect to the balance of power between management and workers. In particular, the erosion of the power of unions and the lack of adequate new forms of organisation that might support the rights of workers is apparent.

Another friction between technologies and labour activists surrounds the issue of access and appropriation of technology such as broadband; an issue that has been discussed by scholars with respect to the digital divide and digital inequality (DiMaggio, Hargittai, Celeste, and Shafer, 2004; DiMaggio, Hargittai, Neuman, and Robinson, 2001; Forlano and Powell, 2011; Hargittai, 2007). According to Karl, a labour advocate in the education sector, social media such as Twitter has been used effectively to organise educators and unions. One particularly successful campaign \#thewholestory, which used a hashtag aimed to correct a series of Chicago Tribune stories about teacher certification, garnered 5 million impressions on Twitter. Yet, while middle class parents are technology-savvy, have broadband access and are engaged in their schools, the majority of people that send their kids to Chicago public schools do not have access to broadband and 160 schools do not even have libraries. Karl says, 'You can push these apps as a boon to democracy but if people don't have access it is not really doing any good.' This illustrates the ways in which labour activists acknowledge how communication technology can open up avenues for 
activism and social change if people have access to it. But it is not enough to have mere access to technology; it is also necessary to have the technological literacy and skills in order to use it effectively and creatively.

This point about technological literacy and skills is well illustrated by a position paper by the Design Studio for Social Intervention, a social justice non-profit organisation in Boston that writes:

what youth of color need is not new technologies (they have them), but equal opportunity to build on these technologies, to deploy their own creativity, and to amplify their entrepreneurial spirit and hustle. Youth of color are ready and willing to be more than consumers: to learn code, use 3-D printers and digital fabrication, "hack" new styles into clothes, create new uses for social media, etc. They need opportunities that build on their skills, knowledge and existing social practices-respecting the ingenuity of current hustles, building on the skills they've taught themselves in regards to entrepreneurship, technology, and networking (Lobenstine and Bailey, 2014: 6).

The organisation points out that many of the social practices that have existed in communities of colour for many years (and been criticised and condemned by mainstream society) are now heralded as boons of the sharing economy. For example, apartment sharing that was once considered illegal has now been reinvented by Airbnb as a symbol of the so-called sharing economy. In this case, social justice organisations such as the Design Studio for Social Intervention are entangled in advancing some of the same philosophies that are deeply embedded into Silicon Valley engineering culture and the United States government's push to train more and more people in Science Technology Engineering and Math (STEM) fields (and simultaneously cut funding to the humanities, the social sciences and the arts). There is considerable debate over whether there is any inherent value in learning to code, design 3-D printed objects, advance disruptive innovation or foster an entrepreneurial spirit (Lepore, 2014; McPherson, 2008). Will learning to code allow communities of colour to obtain higher paying jobs or will it serve to suppress wages due to a larger supply of qualified applicants? It is difficult to argue that youth of colour should be denied the same kinds of technological literacy, skills, and knowledge that have become mainstream among other socio-economic groups.

Overall, our interviews with labour activists as well as recent publications by social justice advocacy organisations highlight the ways in which their own narratives around the role of technology and the future of work are entangled with and, at times, in friction and 
conflict with the philosophies and values embedded in technologies vis a vis the future of work. By understanding these entanglements and frictions more deeply, it is possible to identify opportunities to design technological systems that can negotiate between these multiple philosophies and values. One example of this kind of negotiation with respect to crowdsourcing platforms and digital labour marketplaces is discussed here:

How can Turkers move past these disputes and act together on matters that they do agree on? Just as paid crowdsourcing has reconfigured the dynamics of work, introducing a new form of labor that relies on temporary labor relations and short term tasks, it seems that the distributed nature of the workforce may also be transforming the requirements of labor activism. What would the equivalent of a labor union look like online? (Salehi, Irani, Khatib, and Bernstein, 2014: 3)

Turkopticon and Dynamo are two specific platforms that can be understood as design interventions that embed alternative sets of values and possibilities around labour activism (Irani and Silberman, 2013; Irani and Silberman, 2014; Salehi et al., 2014). In a similar vein, our interviews and background research on the role of technology in the future of work led us to explore the use of participatory design and speculative design in order to find ways of opening up discussions, challenging the depictions of the role of technology in the future of work as portrayed by mainstream media representations, negotiating between multiple philosophies embedded within technologies and labour activism, and developing productive frictions that might lead to ideas for prototypes of technologies that might embed ideals of social justice.

\section{Reimagining Work}

Our participatory and speculative design workshop allowed us to engage in discussions around technology and labour including their historical relationships, the values and philosophies embedded in particular reconfigurations and the ways in which alternate possible futures could be imagined and prototyped. Through the workshop, we sought to position the future as a temporal socio-technical space that was actively becoming and being made by labour advocates with their values and philosophies in mind rather than merely acting upon them as a linear, disruptive and revolutionary force. We aimed to further open up the 'black box' of technology in to a space of engagement, participation and access by labour advocates, while, at the same time, understanding the limitations and constraints of this approach. Specifically, even if technologies are designed 
around particular activist values and technologies, it is difficult to control their adoption, appropriation and use. This is particularly true in the case of labour activism because, as pointed out earlier, to a great extent, workers do not have control over the technologies that they use and those that are used to manage them since many no longer participate in formal labour organisations.

In this section, we will draw examples from the workshop to illustrate the ways in which we engaged with narratives and discourses around the future and technology as well as what we learned about the entanglements and frictions between the philosophies embedded in technology and those in labour activism. With respect to the temporal boundaries of the workshop, the game that we designed stretched from 3000 years in the past to 30 years into the future. After experiencing the expanded time horizon in the game to understand labour organising throughout history including, for example, the histories of slave revolts in Ancient Egypt, Jonathan, a labour advocate, said, 'It is possible to ask what does a collective at the workplace look like 50 years from now...that kind of imaginative exercise allows people to see new possibilities.'

David, the healthcare labour advocate said, 'There is real value in thinking things through... seeing the progression and the interaction historically and concretely in a way. Often, [we] don't do the work of sitting down and examining what has occurred historically.' As part of the game, we asked participants in the workshop to create campaigns, artifacts, uniforms, schedules and technologies as part of building counterfactual histories. These histories were a way of imagining the past that opened up potential conversations about speculating about the future. For example, Penny, a participant from the nonprofit community, suggested that the work done through the game was empowering.

PENNY: There's something kind of empowering about seeing that throughout all these ages, it's a process of workers that are responding to the conditions they find themselves in and even in our little team of two or three people, that in some small way were creatively trying to respond to a challenge of our work situation that was in front of us.

Similarly, the speculative aspect of creating alternative histories inspired another workshop participant:

ALAN: I really liked the term counterfactual. It was humorous and fun but also helps break open some creative energy. And there are counter histories so it's 
sort of thinking about what history are we paying attention to. Are we looking at the social history of conditions of work? Are we looking at industrial history and new forms of production and celebration of technology? So I think maybe it's about just being critical about what are we looking at as history, what constitutes history, and that's what's perhaps helpful. Are we taking inspiration from this history or from this history when we're thinking about current conditions?

The first era represented on the game board was Ancient Greece and Egypt. Players were asked to speculate about what it may have been like to build the pyramids, or to work as a slave in someone's home. Participants agreed that thinking so far into the past was more difficult to them than thinking about the other more recent and familiar eras represented, including the future.

There was a connection between the ability to imagine alternatives to historical narratives and the ability to imagine or recall existing narratives. For example, not knowing the power structures or labour practices of the Egyptians made it difficult for the participants to imagine what a list of demands would look like in Ancient Egypt. Moreover, as one participant pointed out, knowledge of history was not always helpful in opening up new ideas for the past or present. Repeating themes of oppression throughout the history of labour, combined with the hope for change that forms the basis of the work labour advocates do, made it difficult to decide whether to represent a perceived reality or an optimistic future. Threading this difficult needle helped maintain the nuanced balance between the dystopian and utopian extremes that creep into most discussions of the future including the mainstream media representations around the role of technology and the future of work.

JARED: I thought it was sort of hard to balance ideas that were imaginative or optimistic with ones that were pragmatic, especially [unintelligible] because we know what the constraints were. Yeah, trying to think of solutions that are workable vs. ones that are fun.

Though these speculations about the past were challenging, they were successful in illustrating the values and philosophies held by labour advocates through the creation of interventions during the game play. During the prototyping session participants were able to experience the ways in which hands-on material engagement allowed the group's ideas to move forward. For example, Penny noted that she had an expectation that discussions yield insight and decision-making, and that creating something is a result of meticulous planning and discussion. However, during the prototyping activity, she found that her 
group's conversation did not move forward until they began to make their prototype. It was the act of making that enabled them to make decisions and move forward.

PENNY: I was struck by the last exercise, when we started off in our group just talking. We were talking around each other, until you actually came in and intervened and said you must get up and do something. It wasn't until we actually started physically moving that we-we were developing our idea as we were just putting stuff together, and for a linear thinker it is just like how did that happen? We're used to brainstorming...you have to battle out the ideas before you get to any kind of action, and this was actually, you know, doing a physicality that actually helped me think through a problem.

While Penny focused on her surprise to find that decisions were made through actions rather than deliberation. Aiden, a software developer, articulated a similar observation about his expectations and his experience of the prototyping activity, but from a different perspective. He found that the activity provided greater understanding of the complex set of issues surrounding labour and technology, rather than a plan for a product.

\begin{abstract}
AIDEN: The product that came out of this was more of a really good understanding of some of the dimensions of the problem space, more so than actionable prototypes for interventions- which I think is counterintuitive when you hear that you're going to be prototyping in teams. I think a lot, as a software developer, like hackathons are very popular collaborative event. But those are often times too focused on making some kind of product, and then those are seen as the outcome rather than by making something you get an understanding of the dimensions.
\end{abstract}

While Penny's prior experience and her field prompted her to think about process, Aiden's work in software was more product-focused. Despite the focus on process or product, it seems that the aims and outcomes of the activities were inverted with the norm. The convention, for both Penny and Aiden, was that discussion developed thoughts and ideas, and design developed products. They both articulated surprise at the ways in which the design work prompted and enhanced, or shaped, thinking. This transformation toward making as a way of knowing particularly helped Penny's group, as well as Halpern's group, to break out of circular conversations and move toward consensus.

Overall, through the participatory and speculative design workshop, participants were able 
to engage with a reimagining of the relationship between labour and technology based on the values held by labour advocates. The workshop served as a forum in which to imagine the future, breaking out of the day-to-day temporal constraints of policy advocacy as well as challenging some of the established ways of thinking about technology which are well represented in mainstream media debates. Through rigorous discussion both during the game and the prototyping activity a complex and nuanced understanding emerged of how technology and its philosophies are embedded in socio-technical systems. The workshop left us with a number of questions about the entanglements and frictions between the philosophies of labour activists and technologies as well as about possible future directions for our methodology.

\section{Future Directions}

While the participatory and speculative design workshop activities including the game and prototyping exercises were useful in opening up conversations and challenging dominant narratives about technology and the future as well as expressing the values and philosophies of labour activists, we found that there were specific ways in which our goals could have been more explicit. For example, during the wrap up discussion following the workshop, David mentioned that the workshop could have been more explicitly connected to social justice concerns and the conditions of low-income workers. While we expected the labour activists to represent their own positions and constituencies during the game and prototyping activities, it is possible that due to the mixed group of workshop participants, the team members did not share the same values and commitments. Rather than allowing the game and prototyping activities to be relatively open-ended, we might have given the teams more specific constraints and direction. For example, in the game, rather than 'design a schedule' or 'design a logo' for a fisherman in Ancient Greece, each of the game cards could have been directly tied to achieving certain improvements in the lives of workers. In this case, the prompt would need to state more clearly 'design a schedule that offers greater leisure time' or 'design a logo that shows the empowerment of workers.'

Furthermore, our follow up interviews after the workshop suggest that there is great potential to create a version of participatory and speculative design workshop specifically for workers (rather than their labour advocacy intermediaries). This is because workers may have direct experiences with the ways in which technology is shaping their day-to-day work as well as the ways that they are using it for their jobs as well as for social support. However, at the same time, with low-wage jobs, it is extremely unlikely that workers are being consulted when decisions about which technologies to adopt are being made by 
specific employers. Specifically, the labour advocates that we spoke with were supportive of the possibility of using design methods for the purpose of building a greater sense of agency in ongoing socio-technical narratives around work as well as for translating individual experiences of work to broader structural inequalities that are shared by groups of workers. The activists indicated their constituents might find value and agency from the workshop, suggesting it supported meaningful material deliberation.

In keeping with the methodological traditions of participatory design in shaping technologies to benefit workers by incorporating their tacit knowledge, we believe that participatory and speculative design methods offer one way of potentially engaging in discussions around the role of technology in future of work narratives as well as surfacing the entanglements and frictions between labour activists and technologies. However, while participatory design focused primarily on organised groups of workers at the same organisation, we believe that there is value in extending it to distributed and unorganised workers that may share similar experiences but not yet be affiliated with one another in order to build constituencies.

In particular, a design intervention of this kind may be important and useful in situations in which workers are most isolated from one another, such as domestic workers and homecare workers. These individuals often do not identify as "workers" since they do not perform their jobs at a traditional workplace. Domestic workers and homecare workers are some of the lowest paid workers and due to their contingent nature have few protections in the United States under the law. Furthermore, a range of digital technologies such as mobile applications, keycard access, 'nanny cams' and other mechanisms are used to track them at work. As a result, there is potential not only to employ design methods as one means of bringing workers together but also engaging with technology activists to create alternative applications and platforms that could benefit domestic workers and homecare workers directly.

Furthermore, it is critical that future projects enable labour advocates to strengthen their participation with, access to, and literacy about technology through meaningful material engagements. This would allow for a deeper negotiation between the philosophies embedded in labour activism and those present within technologies. This deeper engagement could be mobilised productively to create demonstrations, experiments, and prototypes that could intervene in discourses around the role of technology in the future of work. It could also reveal additional sites of conflict between the philosophies of labour activists and the ways in which technologies are positioned vis a vis the future of work. It is only through this kind of intimacy and entanglement between labour advocates and emerging technologies that it is possible to shift the embedded values in the discourses 
that are continuing to powerfully shape the terms of the debate and the decisions that business leaders and government officials are making when they consider the role of technology in the future of work.

\section{Biographical Note}

Laura Forlano is an Assistant Professor at the Institute of Design at Illinois Institute of Technology. Her research is focused on the intersection between emerging technologies, material practices and the future of cities with a specific focus on emergent forms of organising and urbanism.

Megan Halpern is a Postdoctoral Associate at Arizona State University. Her research interests include collaboration in art, science, and design; human computer interaction design; and public engagement with science. Her recent work has focused on the relationship between experts and audiences in a variety of contexts in both the performing arts and sciences.

\section{Notes}

[1] See https://www.freelancersunion.org

[2] See http://www.kellyservices.com/Global/The_Talent_Project_iPad_App/

[3] See http://www.mbopartners.com/state-of-independence

[4] See http://www.microenterpriseworks.org

[5] See http://www.mbopartners.com/state-of-independence 
[6] See http://www.oxforddictionaries.com/us/definition/american_english/friction

[7] E-mail correspondence with Joseph Thomas Phelan on February 25, 2015.

\section{References}

Andrejevic, Mark. 'Exploitation in the Data Mine', in Christian Fuchs, Kees Boersma, Anders Albrechtslund and Marisol Sandoval (eds). Internet and Surveillance: The Challenges of Web 2.0 and Social Media (London: Routledge, 2012), 71-88.

Autor, David. 'Polanyi's Paradox and the Shape of Employment Growth'. Paper presented at the Federal Reserve Bank of Kansas City, Kansas City (2014).

Barad, Karen. 'Posthumanist Performativity: Toward an Understanding of How Matter Comes to Matter', Signs 28.3 (2003): 801-831.

Barad, Karen. Meeting the Universe Halfway: Quantum Physics and the Entanglement of Matter and Meaning (Durham, NC: Duke University Press, 2007).

Barben, Daniel, Erik Fisher, Cynthia Selin, and David H. Guston. 'Anticipatory Governance of Nanotechnology: Foresight, Engagement, and Integration', in Edward J. Hackett, Olga Amsterdamska Michael Lynch and Judy Wajcman (eds). The Handbook of Science and Technology Studies 3. (Cambridge, Mass: MIT Press, 2007), 979-1000.

Benkler, Yochai. The Wealth of Networks: How Social Production Transforms Markets and Freedom (New Haven, CT: Yale University Press 2006).

Bijker, Wiebe. E., and John Law (eds). Shaping Technology / Building Society: Studies in Sociotechnical Change (Cambridge, Mass: MIT Press, 1994).

Bogost, lan. Alien Phenomenology, or What It's Like to be a Thing. (Minneapolis: University of Minnesota Press, 2012).

Brynjolfsson, Erik and Andrew McAfee. The Second Machine Age: Work, Progress, and Prosperity in a Time of Brilliant Technologies (WW Norton and Company, 2014).

Bruns, Axel and Jean E. Burgess. 'The Use of Twitter Hashtags in the Formation of Ad Hoc Publics'. In 6th European Consortium for Political Research General Conference, 25 - 27 August 2011, University of Iceland, Reykjavik (2011).

Burgess, Jean, Marcus Foth and Helen G. Klaebe. 'Everyday Creativity as Civic Engagement: A Cultural Citizenship View of New Media', in Communications Policy \& Research Forum, Sep 25-26, Sydney (2006).

Burgess, Jean, and Joshua Green. YouTube: Online Video and Participatory Culture (John 
Wiley and Sons, 2013).

Davidow, William H. and Michael S. Malone. 'What Happens to Society When Robots Replace Workers'? Harvard Business Review 10 December (2014).

Davies, Sarah. R., Cynthia Selin, Gretchen Gano, and Ângela. G. Pereira. 'Citizen Engagement and Urban Change: Three Case Studies of Material Deliberation', Cities 29.6 (2012): 351-357.

DiMaggio, Paul, Eszter Hargittai, Coral Celeste, and Steven Shafer. 'Digital Inequality: From Unequal Access to Differentiated Use', Social Inequality (2004): 355-400.

DiMaggio, Paul, Eszter Hargittai, W. Russell Neuman, and John P. Robinson. 'Social Implications of the Internet', Annual Review of Sociology (2001): 307-336.

DiSalvo, Carl. 'Design and the Construction of Publics', Design Issues 25.1 (2009): 48-63.

DiSalvo, Carl. Adversarial Design. (Cambridge, Mass: MIT Press, 2012).

DiSalvo, Carl, Thomas Lodato, Laura Fries, Beth Schechter and Thomas Barnwell. 'The Collective Articulation of Issues as Design Practice', CoDesign 7.3-4 (2011): 185-197.

Dourish, Paul, and Genevieve Bell. Divining a Digital Future: Mess and Mythology in Ubiquitous Computing (Cambridge, Mass: MIT Press, 2011).

Dourish, Paul, and Melissa Mazmanian. 'Media as Material: Information Representations as Material Foundations for Organisational Practice'. Paper presented at the Third International Symposium on Process Organisational Studies, Corfu, Greece (2011).

Fitzpatrick, Kathleen. Planned Obsolescence: Publishing, Technology, and the Future of the Academy (New York: NYU Press, 2011).

Flanagan, Mary, and Helen Nissenbaum. Values at Play in Digital Games (Cambridge, Mass: MIT Press, 2014).

Forlano, Laura. 'Anytime? Anywhere?: Reframing Debates Around Community and Municipal Wireless Networking', Journal of Community Informatics 4.1 (2008).

Forlano, Laura and Alison Powell. From the Digital Divide to Digital Excellence: Global Best Practices for Municipal and Community Wireless Networks (Washington, DC: New America Foundation, 2011).

Foth, Marcus, Laura Forlano, Martin Gibbs, and Christine Satchell. From Social Butterfly to Engaged Citizen: Urban Informatics, Social Media, Ubiquitous Computing, and Mobile Technology to Support Citizen Engagement (Cambridge, Mass: MIT Press, 2011).

Frey, Carl Benedikt and Michael A. Osborne. 'The Future of Employment: How Susceptible are Jobs to Computerisation'? Paper presented at the Machines and Employment Workshop, Oxford, England (2013).

Friedman, Batya, and Helen Nissenbaum. 'Bias in computer systems', ACM Transactions on Information Systems (TOIS) 14.3 (1996): 330-347. 
Gaver, Bill, Tony Dunne and Elena Pacenti. 'Design: Cultural Probes', Interactions 6.1 (1999): 21-29.

Gibson, J.J. 'The Theory of Affordances', in Robert Shaw and John D. Bransford (eds). Perceiving, Acting and Knowing: Toward an Ecological Psychology (New York: Wiley, 1977), 67-82.

Gleason, Carrie and Susan Lambert. 'Uncertainty by the Hour', Future of Work Project (Open Society Foundations, 2014).

Gordon, Eric, and Gene Koo. 'Placeworlds: Using Virtual Worlds to Foster Civic Engagement', Space and Culture 11.3 (2008): 204-221.

Hardt, Michael. 'Affective Labor', Boundary 2 (1999): 89-100.

Hargittai, Eszter. 'Whose space? Differences Among Users and Non Users of Social Network Sites', Journal of Computer Mediated Communication 13.1 (2007): 276-297.

Head, Simon. Mindless: Why Smarter Machines are Making Dumber Humans (Basic Books, 2014).

Hilgartner, Stephen. 'The Dominant View of Popularization: Conceptual Problems, Political Uses', Social Studies of Science 20.3 (1990): 519-539.

Hillgren, Per-Anders, Anna Seravalli and Anders Emilson. 'Prototyping and Infrastructuring in Design for Social Innovation', CoDesign 7.3-4 (2011): 169-183.

Irani, Lilly. 'Justice for "Data Janitors", Public Books, 15 January (2015), http://www.publicbooks.org/nonfiction/justice-for-data-janitors

Irani, Lilly and M. Six Silberman. Turkopticon: Interrupting Worker Invisibility in Amazon Mechanical Turk. Paper presented at the Proceedings of the SIGCHI Conference on Human Factors in Computing Systems (2013).

Irani, Lilly and M. Six Silberman. 'From Critical Design to Critical Infrastructure: Lessons from Turkopticon', Interactions 21.4 (2014): 32-35.

Joss, Simon. 'Introduction: Public Participation in Science and Technology Policy and Decision Making-Ephemeral Phenomenon or Lasting Change'? Science and Public Policy 26 (1999): 290-293.

Latour, Bruno. 'Where are the Missing Masses? A Sociology of Few Mundane Objects', in Wiebe E. Bijker and John Law (eds). Shaping Technology/Building Society. Studies in Sociotechnical Change (Cambridge, Mass: MIT Press, 1992), 151-180.

Latour, Bruno. 'On Actor-Network Theory: A Few Clarifications', Soziale Welt (1996): 369_ 381.

Latour, Bruno. Reassembling the Social: An Introduction to Actor-Network-Theory (Oxford: Oxford University Press, 2005). 
Law, John. After Method: Mess in Social Science Research (London: Routledge, 2004).

Lazzarato, Maurizio. 'Immaterial Labour', in Paulo Virno and Michael Hardt (eds). Radical Thought in Italy: A Potential Politics ((Minneapolis: University of Minnesota Press, 1996), 133-147.

Leonardi, Paul M. 'Digital Materiality? How Artifacts Without Matter, Matter', First Monday 15.6 (2010) http://firstmonday.org/article/view/3036/2567

Lepore, Jill. 'The Disruption Machine: What the Gospel of Innovation Gets Wrong', The New Yorker, June 23 (2014), http://www.newyorker.com/magazine/2014/06/23/the-disruptionmachine

Lobenstine, Lori and Kenneth Bailey. 'Redlining the Adjacent Possible: Youth and Communities of Color Face the (Not) New Future of (Not) Work', Future of Work Project (Open Society Foundations, 2014).

McDonough, William and Michael Braungart. Cradle to Cradle: Remaking the Way We Make Things (New York: North Point Press, 2002).

McPherson, Tara. 'A Rule Set for the Future', in Tara McPherson (ed.). Digital Youth, Innovation, and the Unexpected (Cambridge, MA: MIT Press, 2008), 1-26.

Mouffe, Chantal. 'Pluralism, Dissensus and Democratic Citizenship', II Seminário Internacional Educação Intercultural, Gênero e Movimentos Sociais. Identidade, Diferença, Mediações (2003): 1-10.

Nissenbaum, Helen. 'How Computer Systems Embody Values', Computer 34.3 (2001): 117-119.

Norman, Donald A. The Design of Everyday Things (New York: Doubleday 1990).

Orlikowski, Wanda J. 'Sociomaterial Practices: Exploring Technology at Work', Organisation Studies 28.9 (2007): 1435-1448.

Parikka, Jussi. 'New Materialism as Media Theory: Medianatures and Dirty Matter', Communication and Critical/Cultural Studies 9.1 (2012): 95-100.

Pinch, Trevor J. and Wiebe E. Bijker. 'The Social Construction of Facts and Artefacts: Or How the Sociology of Science and the Sociology of Technology Might Benefit Each Other', Social Studies of Science 14.3 (1984): 399-441.

Tsing, Anna Lowenhaupt. Friction: An Ethnography of Global Connection (Princeton, NJ: Princeton University Press, 2005).

Rheingold, Howard. 'Using Participatory Media and Public Voice to Encourage Civic Engagement', in W. Lance Bennet (ed.). Civic Life Online: Learning How Digital Media Can Engage Youth (Cambridge, Mass: MIT Press 2008): 97-118.

Rise of the Robots. The Economist, 29 March (2014), http://www.economist.com/printedition/2014-03-29 
Robles, Erica and Mikael Wiberg. 'From Materials to Materiality: Thinking of Computation from Within an Icehotel', Interactions 18.1 (2011): 32-37.

Salehi, Niloufar, Lilly Irani, Ali Al Khatib and Michael Bernstein. 'Dynamo: Designing Interactive Technology to Support Social Movements in Digital Labor', Future of Work Project (Open Society Foundations, 2014).

Scholz, Trebor. Digital Labor: The Internet as Playground and Factory (New York: Routledge, 2012).

Spinuzzi, Clay. 'The Methodology of Participatory Design', Technical Communication 52.2 (2005): 163-174.

Star, Susan Leigh. 'The Ethnography of Infrastructure', American Behavioral Scientist 43.3 (1999): 377-391.

Suchman, Lucy. 'Anthropological Relocations and the Limits of Design', Annual Review of Anthropology 40 (2011): 1-18.

Terranova, Tiziana. 'Free Labor: Producing Culture for the Digital Economy', Social Text 18.2 (2000): 33-58.

Tufekci, Zeynep. 'Failing the Third Machine Age: When Robots Come for Grandma', Medium, July 22 (2014), https://medium.com/message/failing-the-third-machine-age1883 e647ba74

Wynne, Brian. 'Public Engagement as a Means of Restoring Public Trust in Science-Hitting the Notes, but Missing the Music'? Community Genetics 9.3 (2006): 211-220.\#\#\#\# 
The LOCKSS System has the permission to collect, preserve and serve this open access

Archival Unit

\section{OPEN HUMANITIES PRESS}

The Fibreculture Journal is published by The Fibreculture Journal Incorporated in partnership with Open Humanities Press. 


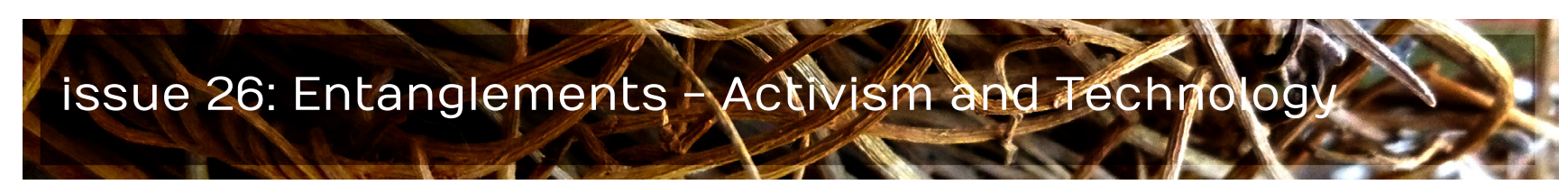

\title{
FCJ-190 Building a Better Twitter: A Study of the Twitter Al- ternatives GNU social, Quitter, rstat.us, and Twister
}

Robert W. Gehl

The University of Utah

\begin{abstract}
:
Drawing on interviews with developers and close readings of site interfaces and architectures, this essay explores four Twitter alternatives: Twister, rstat. us, GNU social (a Free Software Foundation microblogging software project) and Quitter (a specific installation of GNU social). The interviews and analyses of these Twitter alternatives reveal how these developers relate their projects to mainstream social media architectures and cultures; how they conceive of Twitter's development over time; how they think about legal issues as they make their alternatives; and whether or not they conceive of their work as activism. In sum, I explore how these developers are critically reverse engineering Twitter and how that process intersects with multiple concerns and tensions, and how these tensions are generating new ways to think about what it means to 'do' microblogging.
\end{abstract}

doi: $10.15307 / f c j .26 .190 .2015$ 


\section{Introduction: Universalised Twitter Meets Its Alternatives}

Anna Tsing's Friction: An Ethnography of Global Connection (2005) explores the moments when a universalised practice (for example, global capitalism) gets a grip on a local context (for example, in an Indonesian rain forest). When the slippery universal, which in some circles might be lauded as 'frictionless' (as in 'frictionless capitalism'), meets local contexts, frictions occur. Friction traces the move from the desire for frictionless ideals to its awkward and messy contact with situated realities. Friction is resistance, but it is also productive and necessary for any energetic system.

Taking up this idea, this paper traces the friction between Twitter and Twitter alternatives. In this sense, Twitter is situated as 'universalised' (Koopman, 2013: 19), which is to say that it is now an idealised form of online communication. Twitter has established a format of online communication-microblogging-that is seen to simultaneously support free expression yet also is amenable to surveillance capitalism. As such, it has a significant impact on media politics; it is a site by which people socialise, promote themselves, make jokes, build brand identities, make tech investments (for example, purchase Twitter stock), or organise activists. Whereas its early history was marked by commentary on the oddities of 140-character communication and the banality of posting mundane moments of one's day, Twitter is now second nature for billions of people worldwide as a part of daily life. The use of the octothorpe (\#) in hashtags is now part of language, as when someone leaving a party says 'hashtag I'm out', evoking '\#imout.' Although Twitter has not been universalised to the extent that capitalism has, there is a lot of energy devoted to spreading Twitter, as in the case of the U.S. State Department's support of the service due to its perceived use in protest movements outside the United States (Bratich, 2011).

However, Twitter is not without its critics. This is reflected by the fact that there is a robust collection of critical alternatives to Twitter being developed. This paper presents four of these alternatives and considers the frictions that occur when universalised Twitterstyle microblogging meets these particular alternative practices. Specifically, drawing on the approach of critical reverse engineering, this paper considers four points of contact between Twitter and the alternatives: the pragmatic, the genealogical, the legal, and the normative. Carefully studying Twitter alternatives at each of these moments of contact reveals both their powerful critiques of Twitter as well as their specific implementations of the format of microblogging. Through analysis of these moments of contact between Twitter and its alternatives, we will see varying frictional practices: cross-posting versus separation; shifts in conceptions of Twitter's role in the larger political economy of the Internet; different approaches to the legal questions of reverse engineering; and even a tension over whether making a Twitter alternative is media activism or not. Doing this work 
tells us much about Twitter, but it also shows that there is much more to microblogging than Twitter.

The data for this work are drawn from interviews with the developers of these alternative sites [2] and to a lesser extent from software studies-inspired (Fuller, 2008; Mackenzie, 2006; Kitchin and Dodge, 2011; Chun 2011) close readings of the architectures, coding languages, and interfaces of the sites. The interviews and analyses of Twitter alternatives are read through a frame of 'critical reverse engineering,' an approach to studying the relationship between an original technical artefact (in this case, Twitter) and artefacts built in critical response to that artefact (here, rstat.us, GNU social, the Quitter implementation of GNU social [1], and Twister). Before exploring the alternatives, I turn to that framework.

\section{Critical Reverse Engineering}

In previous work (Gehl, 2014a, 2014b), I draw on the literature of reverse engineering as it appears in the fields of law and engineering (e.g., Ingle, 1994; Samuelson and Scotchmer, 2002; Eilam, 2011) to lay out 'critical reverse engineering': a theoretical and methodological framework for the study of social media alternatives. Critical reverse engineering is a process by which we can take apart and analyse the technical artefacts we confront. I am particularly concerned about how this process can be used to critique mainstream social media sites such as Facebook and Twitter. However, this process does not stop at critique; the goal is to use the knowledge gleaned from the analysis of the social media site to make better systems. It thus intersects with-but does not necessarily map directly ontothe specific form of activism of making media alternatives (e.g., Atton, 2002; Couldry and Curran, 2003; Rodriguez, 2001).

I argue that there are four key points of contact included in this approach: pragmatic, genealogical, legal, and normative. First, critical reverse engineers are pragmatic in that they retain much of the functionality, design, and generic practices of the mainstream sites. For example, the social media alternative Ello relies on the follower/followed relationship developed by Twitter as well as a 'stream' of new content (with new content on top) popularised by Facebook. The pragmatic response of alternative developers is to avoid reinventing the format of social media wholesale and thus alienating would-be users. Rather, this example of 'making do,' is a moment of friction between an ideal (a whole new system) and the 'compromise of practical action' (Tsing, 2005: 85). Alternative builders attempt to mix familiar design conventions with new functionalities and modes of connection (Gehl, 2014b: 19). This reflects canonical reverse engineering practice. 
Second, social media alternative makers reverse engineer mainstream sites by doing what Foucault might call the 'gray, meticulous, and patiently documentary' (Foucault, 1984: 76) work of genealogical analysis of the mainstream sites. Reverse engineers look at many artefacts to understand the object they are reverse engineering: documentation, white papers, press releases, how the organisations that produce artefacts are structured, the theories of the user deployed by artefact makers, previous versions of the artefacts, databases, specifications, and of course lines of code. This is an intensely analytical practice. Moreover, alternative social media sites very often replicate the culture, practices, and interfaces of mainstream sites before mainstream sites become highly commercialised. Thus the alternative developers think historically, and look to the past to construct a new social media future.

Next is the legal orientation. Much as with fair use exceptions in copyright law, many liberal legal regimes recognise a reverse engineering exception in intellectual property law. As I argue elsewhere, 'in terms of the functional side [of software], unless it has been protected by a patent, we are free to replicate the functionality of existing software. And in order to do that, we are in fact allowed to open up, probe, test, and disassemble software-even to make copies of it in the process' (Gehl, 2014b: 8). While this exception varies in each legal context (say, the United States versus Australia) and over time (especially as technology corporations engage in legal fights to limit these practices), reverse engineering is a longstanding, legal practice in manufacturing, engineering, design, and technological development. Social media alternatives thus enjoy some legal protections, even as they replicate many features of mainstream sites. This is also enhanced when the alternatives draw on the traditions of copyleft, the Free Software movement, and Creative Commons, which have for decades used copyright law against itself (Weber, 2004; Coleman, 2009).

Finally, as I ask elsewhere, 'why build a Twitter alternative unless one has a goal in mind' (Gehl, 2014b: 18)? Drawing on the reverse engineering literature, I argue that taking apart social media is only the first step. The goal of reverse engineering is always to use the knowledge produced during the process of taking apart an object to build a new object. Similarly, critical reverse engineering is normative; it does not stop at critique, but rather must continue on to the development of alternatives that improve upon the originals. In this sense, it aligns well with Andrew Feenberg's (1986) recommendation that critical work be grounded in material reality, seek contradictions in that reality, and use such contradictions to make a better world. Builders of alternatives are doing more than decrying what they see as the problems of mainstream social media; they are working to build better systems. 


\section{Critiquing Twitter: GNU social, Quitter, rstat.us, and Twister}

\begin{tabular}{|c|c|c|c|c|c|c|}
\hline $\begin{array}{l}\text { Alternative } \\
\text { Site Name }\end{array}$ & URL & Interviewee(s) & Position(s) & Location & Features & License \\
\hline rstat.us & https://rstat.us/ & $\begin{array}{l}\text { Carol Nichols } \\
\text { and David } \\
\text { Wilkinson }\end{array}$ & Co-founders & USA & $\begin{array}{l}\text { Cross-posting to } \\
\text { Twitter; Federated } \\
\text { Structure }\end{array}$ & $\begin{array}{l}\text { Creative } \\
\text { Commons } 0\end{array}$ \\
\hline Quitter & https://quitter.se & $\begin{array}{l}\text { Hannes } \\
\text { Mannerheim }\end{array}$ & Co-founder & Sweden & $\begin{array}{l}\text { Cross-posting to } \\
\text { Twitter; Federated } \\
\text { Structure }\end{array}$ & GNU Affero \\
\hline GNU social & $\begin{array}{l}\text { https://gnu.io/ } \\
\text { social/ }\end{array}$ & $\begin{array}{l}\text { Matt Lee and } \\
\text { Donald } \\
\text { Robison }\end{array}$ & Co-founders & USA & $\begin{array}{l}\text { Cross-posting to } \\
\text { Twitter; Federated } \\
\text { Structure }\end{array}$ & GNU Affero \\
\hline Twister & $\begin{array}{l}\underline{\mathrm{http}: / /} \\
\underline{\text { twister.net.co/ }}\end{array}$ & Miguel Freitas & Founder & Brazil & $\begin{array}{l}\text { Peer-to-peer } \\
\text { structure; no cross- } \\
\text { posting to Twitter }\end{array}$ & MIT/BSD \\
\hline
\end{tabular}

Figure 1: The four Twitter alternatives profiled in this paper.

By simply positioning themselves as Twitter alternatives, rstat.us, Twister, GNU social (and its specific implementation Quitter) all present an implicit critique of Twitter. These implicit critiques were made explicit in the interviews I conducted with alternative makers. For example, in interview Carol Nichols of rstat.us faulted Twitter for the following:

- the ever-increasing amount of ads

- the lack of granular privacy/blocking/harassment reporting features

- the possible compliance of Twitter with law enforcement/government agencies in revealing even private account information

- weird [user interface] decisions they make like the blue conversation threading

- the hostility Twitter has towards 3rd party app developers

- ...putting tweets from people you don't follow in your timeline

- [their intention to] start curating your timeline for you like Facebook does in the near future

All of the developers interviewed expressed similar concerns, as well as criticisms of Twitter's centralisation and the lack of user control over data and the underlying code. But of course, these critiques of Twitter are well documented in critical social media (as well as, increasingly, in the popular press). By building new Twitter-like systems, these developers are doing more than criticising; they are critically reverse engineering Twitter. I will explore this using the four points of contact of critical reverse engineering, paying special attention to the frictions that occur between and among Twitter and these alternatives as these sites attempt to build a better Twitter. 


\section{Pragmatic}

By simply looking at the interfaces of rstat.us, Twister, and GNU social (including Quitter), it is clear that these Twitter alternatives all retain the universalised, generic structure of 'microblogging': short posts with a character limit, a follower/followed social interaction system, new posts on top (often with a notification that new posts are available), a predominantly single-column presentation on the screen, and a requirement that users sign up for an account. In other words, the alternatives very much replicate the canonical features of Twitter, reflecting Twitter's dominance as a universalised microblogging system. The developers of these sites acknowledge the close ties their alternatives have with Twitter's interface. Carol Nichols notes that rstat.us is 'pretty clearly inspired by Twitter.' Donald Robertson, a member of the Free Software Foundation who helped in early days of GNU social, likewise says, 'the current functionality of GNU social and Twitter are similar.' As I argued above, such a move is pragmatic; maintaining familiar interfaces and functionalities allows users to immediately recognise what these sites are intended for.

Interface similarities do not tell the full story, however. A contrast emerges when we consider how closely these alternatives are entangled with Twitter. GNU social and rstat.us maintain a relatively close tie to Twitter by allowing users to 'cross-post' posts. This means that if a user has a Twitter account and allows rstat.us or GNU social access to Twitter via Twitter's Application Programming Interface (API) the user's posts will simultaneously appear in the connected Twitter account. This allows users to use the alternative site without feeling as if they have left Twitter (and all of the connections they have made in that site). As Matt Lee of GNU social explains, 'everyone using Twitter today could make a switch to GNU social and keep using Twitter. A lot of the apps that post to Twitter can be used too.' Carol Nichols of rstat.us provides more details:

Rstat.us implements Twitter Oauth so that you can sign into rstat.us using your Twitter account instead of creating a new account on rstat.us, if you want. If you do that, you can also syndicate your posts from rstat.us to Twitter so that you can post both places at once.

In addition to allowing the user to cross-post, using Oauth allows a user to use Twitter as the authentication system for the alternative. Tapping into the Twitter API thus makes it easier for users to switch because they can keep and maintain their Twitter accounts. 
However, this use of the Twitter API has a drawback: it reinforces Twitter's central position in microblogging. After all, users who switch to GNU social or rstat.us do not have leave Twitter, and thus they can effectively use these alternatives as third party 'apps' to connect to Twitter (in the same manner as apps like Tweetbot or Ubersocial). Thus, although users might have their Twitter use mediated by an alternative, they are still contributing to Twitter as free labourers (Terranova, 2000).

In contrast, Twister, a peer-to-peer system developed by Miguel Freitas, does not allow for cross-posting. Instead of allowing users to maintain their Twitter connection via an alternative, Twister requires them to sever the connection. In our interview, Freitas noted the decision to not allow cross-posting between Twister and Twitter was a legal one, an interpretation of the Twitter Terms of Service that holds cross-posting is prohibited (also see jpfox, 2014). Setting aside this legal question, one result of this decision is that users who want to keep using Twitter while using Twister would effectively have to work with two systems.

Thus, it is unwise to homogenise Twitter alternatives in terms of their pragmatic relationships to Twitter. Instead, the pragmatic orientation of critical reverse engineering prompts us to consider how loosely or closely tied an alternative is to the mainstream site. The varying degrees of loose or close coupling produce different ways of thinking about what it means to make a Twitter alternative. Does the alternative act as a layer on top of the existing Twitter infrastructure? Or does it ask users to make a complete break with Twitter?

This can be illustrated in a slightly different way with a vivid metaphor supplied by Hannes Mannerheim of Quitter during an interview:

Twitter is the heroin and Quitter is the methadone. Quitter does not aim to be a utopian social media, we're aiming to create a way to drop out, to quit. Maybe later, when enough people have quit.... maybe then we can start transforming the network into something more useful (it will probably happen automatically then). But until then I think we have to mimic Twitter pretty closely, so the threshold to quitting is lower.

Recall that Quitter allows for cross-posting and one can see that this metaphor, where Twitter is the addictive opiate and Quitter the synthetic opioid, is one way to think about leaving Twitter behind. Quitter is a specific implementation of GNU social (a larger, free 
software project) that features a very close copy of Twitter's interface (software called 'Qvitter'). Quitter thus provides a gentle way to kick the Twitter habit. GNU social and rstat. us's cross-posting feature can help with weaning users off Twitter, as well. Twister, on the other hand, is more like going 'cold turkey,' that is, leaving Twitter abruptly. In this sense, the friction emerging as alternatives confront Twitter and either take advantage of Twitter's API or not is productive, resulting in multiple ways to leave Twitter and thus multiple ways to think about microblogging as a more heterogeneous online practice.

\section{Genealogical}

Reverse engineering can be conceived of temporally, because it reverses the forward engineering process of starting with an abstraction (such as a design, a blueprint, or a concept) and implementing that abstraction in concrete material. Reverse engineering thus starts with a concrete artefact and works backwards towards abstractions (Ingle,1994: 9; Chikofsky and Cross, 1990: 15). While reverse engineers don't tend to think of this as a historical process, I suggest that it can be conceived of as such (Gehl, 2014a: 10-11). This is because a large part of the work of reverse engineering is tracing the genealogy of any given artefact, considering how it developed over time, looking at statements designers made about it, comparing new versions to old ones, and ultimately rearticulating the technology back into larger historical and cultural contexts.

The technical details of this genealogical process did not come up much in interviews, but it is readily seen if we dig into online forums dedicated to these Twitter alternatives (especially on the code repository GitHub). For example, a discussion regarding potential cross-posting from Twister to Twitter (jpfox, 2014) led to a discussion of the evolution of Twitter's Terms of Service and API. Twister contributor toyg noted:

One of the clauses in [third party Twitter] applications' standard TOS is that you will not use the app to synchronise with other social networks, end-ofstory. This is a policy [Twitter] implemented in the last 2 years as part of their big 'crush 3rd party clients \& other social networks' strategy - they want people to use Twitter official clients and keep Twitter content inside Twitter.... ...they disabled public RSS feeds, so you need an API key to do pretty much anything.... This is why nobody does any Twitter integration anymore, except for the occasional post-update-to-Twitter from non-social applications (jpfox, 2014). 
In less technical terms, as toyg pointed out to other Twister developers, Twitter once had a very open API-not to mention an RSS feed-which would allow users and developers easy access to the data within Twitter. However, in August 2012, Twitter announced sweeping changes to the API, severely limiting access to users and developers (Sippey, 2012). Several of the developers I talked to did note the changes in Twitter's API as key prompts for their wanting to build alternatives. Thus, as they seek to critically reverse engineer Twitter, the alternative builders are thinking-at least in part-historically, considering Twitter as it has evolved both as a technology, comparing its present-day documentation to previous versions to explore how Twitter's relationship with third-party developers has changed over time.

In addition, the alternative builders also consider Twitter as an online practice embedded in larger historical changes. Dave Wilkinson of rstat.us discussed Twitter as it was in 2010: 'Looking at Twitter toward the end of 2010 and seeing how it was being used as a communication medium (much like Fidonet and modem federation before it during Soviet crises [sic.]) throughout the Arab world was really inspiring.' Here, Wilkinson links Twitter (at least as it appeared in 2010) to previous alternative media that played a role during political protests.

Similarly, Miguel Freitas, who is Brazilian, discusses his observation of the role of social media during that country's Movimento Passe Livre protests in 2013. He noted that social media provided:

A feedback mechanism that [made] these movements grow over time. ... That was something I could clearly see happening here in Brazil, by noticing how the presence on the streets was amplified when people had access to videos showing ... police violence as totally disproportional to the original protesters (against bus fares increases).

Freitas explains that such videos would never air on Brazilian mass media. However, he also noted that this progressive aspect of social media as an alternative to corporatecontrolled mass media is endangered:

While social media as we have today is certainly more empowering to individuals and less prone to censoring than conventional media, there is simply no guarantee that it will keep this way. In fact, we have ... some examples (especially from Facebook) where content selection is already heavily filtered. So, in the long run, these sites might be no better than mass media. 
Another inspiring event: You surely remember London riots, when the government considered shutting down Twitter and Facebook. So it's not about the merit of London rioters, but the mere possibility of shutting down an important channel of information like that that strikes me. This is totally against the idea of the Internet, where you are supposed to have no single point of failure.

In addition to critiques of Twitter's centralisation (like Freitas offers above), the alternative builders have critiqued the evolution of its business model. As Carol Nichols of rstat.us notes:

Especially since Twitter has gone public, and really since they started taking [venture capital], it's to be expected that they will always be making changes that will make them the most money, not those that will make their users the most happy and safe.

And Wilkinson adds that Twitter's Terms of Service changes drew attention to rstat.us as a less exploitative alternative.

In these genealogical accounts, Twitter is presented as having fallen from grace. Twitter was once an open system, with an API that allowed for heterogeneous developers to build on the platform and an interface that allowed any user to circumvent censorship and get messages out. Twitter was part of the social media challenge to centralised media power. However, for Freitas, Wilkinson, and Nichols, Twitter has succumbed to the dictates of surveillance capitalism, both by eschewing activist uses of the service in favour of advertiser-friendly practices and by remaining centralised and thus vulnerable to state surveillance and control.

However, this isn't the only way to trace Twitter's history. During our interview, Hannes Mannerheim of Quitter offered a different take on Twitter: it was never revolutionary. 'In 2010 we [Mannerheim and other Swedish Internet activists] saw how a counterrevolution had started. Spotify started taking users from Bittorrent, Facebook and Twitter had already taken over much of the diverse blogging communities.' In other words, Mannerheim argues that Twitter's highly centralised structure 'took over' from blogging as the dominant social media practice. The existence of Wordpress.com, Livejournal, and Blogger notwithstanding, blogging has traditionally been a distributed format of online communication because blogging software can be run on one's own server (Siles, 2011). Twitter's 'counter-revolution' was to draw in and enclose (Andrejevic, 2007; Boyle, 
2003) distributed practices such as blogging. Thus, for Mannerheim, Twitter was never revolutionary; from its earliest days it was built to counter the revolutionary aspects of peer-to-peer software and 'diverse blogging communities.'

Once again, there is a friction as universalised Twitter meets its alternatives, this time appearing at the genealogical point of contact. The stories the Twitter alternative makers tell about their services and Twitter itself present histories and counter-histories that complicate our understanding of universalised microblogging. For example, when the next social network captures the public imagination-as Ello briefly did in 2014-we are armed with these histories to help us interpret and struggle over the meaning of a new system. Can we read it as a potentially revolutionary system that must be protected from the dictates of surveillance capitalism? Or is the new system simply a corporate reaction to more dangerous practices such as distributed media making? The divergent genealogies of Twitter offered by the alternative makers gives us material for debates about new social media technologies.

Regardless of this divergence, all of the developers are reverse engineering in the sense that they start with a concrete artefact (Twitter as it appears today) and work backwards to abstract architectures and concepts (open APIs, less constrictive TOSs, distributed networking, free software and culture, and alternative media) that they either see in past iterations of Twitter or Internet communication in general. They then seek to re-implement those abstract features into specific, divergent, concrete forms within their alternatives.

\section{Legal}

The legal aspects of the Twitter alternatives are quite important, and the developers discussed them at length in our interviews. Indeed, when Twitter meets the alternatives at the contact-point of the law, many complex practices and discourses emerge. Specifically, three threads emerge that tie in with critical reverse engineering: the legal protection afforded to those who reverse engineer, the legal protections that come from using copyleft, free software, or public domain licenses, and, to a lesser extent, the need to fight for the right to reverse engineer in courts.

The first thread that emerges here is protection due to the legality of reverse engineering. Reverse engineering enjoys a history of legal protections. As legal scholar Pam Samuelson 
has argued, courts have long recognised the "right to take the purchased product apart, measure it, subject it to testing, and the like. The time, money, and energy that reverse engineers invest in analysing products may also be a way of "earning" rights to the information they learn thereby' (Samuelson and Scotchmer, 2002: 1583). This extends to software and computer systems. However, software companies attempt to limit reverse engineering through terms of service (TOS) agreements. Twitter is no exception to this practice. [3] Therefore, the developers of the Twitter alternatives walk a fine line between learning how Twitter works through intense analysis of it on the one hand and violating its TOS on the other. As Nichols of rstat.us notes:

We've been really careful not to violate Twitter's TOS, even in gray areas. I could definitely see them trying to sue us, if they decide to patent 'posting text on the Internet in 140 character segments' or something, which I hope wouldn't be patentable but Amazon has one-click buying patented so who knows.

To protect themselves from lawsuits and accusations of violating intellectual property, the alternative builders use time-tested techniques of building from broad specifications, rather than directly copying Twitter functionality. As Wilkinson says, 'I'm not particularly concerned [about Twitter suing rstat.us]... The technology we use underneath [rstat. us] is implemented from a specification that is well-known and acknowledged by many social-based companies. Google uses it and in fact maintains some of the specifications.' Similarly, Freitas describes the 'clean room implementation' of Twister:

I hired this guy... to implement the [user interface] in HTML/Javascript. He is also a graphic designer. So I described him the interface I wanted in details: how posts should expand on click, where to put the reply box, what to show on mouse hover, the modal dialogs etc. A lot of this functionality is common to both Twitter and other existing microblogging, so l've sent him some screenshots to demonstrate what I meant, but I specifically asked him NOT to use any code or graphic elements from them. He had to work solely based on my specifications and he was free to design the new graphical identity..

In other words, Freitas and the implementer worked from functional specifications that Freitas crafted, rather than attempting to explicitly copy the look and feel of Twitter. Thus, whereas alternatives such as Twister or rstat.us bear many similarities to Twitter, their production follows accepted reverse engineering practices and thus is likely protected by law. 
The second thread is protection due to the 'legal jujitsu' of copyleft, free software licenses, or the public domain. Besides the longstanding protections afforded to reverse engineering in Western law, these Twitter alternatives also enjoy the protection of decades-old practices of copyleft, free licenses, or public domain licensing. Copyleft, inaugurated by software producer and activist Richard Stallman, uses copyright law against itself, licensing objects in such a way that they will always remain freely modifiable to future users. Moreover, copyleft requires anyone who builds on previous copyleft-licensed software to have his or her contribution licensed in the same manner as the original object. Thus, copyleft creates an infrastructure of free and open artefacts, and it is nearly impossible to make them proprietary and closed after they are licensed with a copyleft license. While not copyleft, free software licenses allow users the ability to access, modify, and distribute code (but it does not require them to perpetuate that license on any code they derive from the original project). Licensing an object as public domain allows end users to do whatever they like with the object. This includes creating closed, proprietary versions. However, unlike copyleft, licensing as public domain signals the complete rejection of the copyright system altogether, and hence it is a political move.

The developers of Twitter alternatives are well versed in the use of licenses as a means to protect their projects, but they use different licenses to reflect different political commitments. For example, licensed under the GNU Affero license, GNU social (and hence Quitter's interface Qvitter since it is built on GNU social) enjoys the most copyright protections of the alternatives. The Affero license is copyleft-that is, it allows users to modify GNU social but it requires any derivatives made from it to be licensed the same way. Moreover, it is built specifically for networked software.

Citing Twister's MIT/BSD license (a free software license), Freitas argues that Twister is protected from most, if not all legal procedures meant to shut it down:

I think any legal procedure against me (either because of hypothetical patents or name copyright) is mostly useless. They won't be able to make the source code illegal, nor I think they would be able to shutdown the whole thing even if I quit.

In other words, by using a free software license, Freitas is confident that Twister would live on even with legal actions brought against him. 
As for rstat.us, the license has changed over its development, but the developers always sought to put rstat.us in the public domain. Carol Nichols led the change in rstat.us's license from WTFPL (an acronym for 'Do Whatever the Fuck You Like Public License'), which is, as its name implies, very permissive to Creative Commons 0 - No Rights Reserved, which places rstat.us code and graphics in the public domain (Nichols, 2012a). Whereas WTFPL would essentially do the same as CCO, the former 'sounds like a vaguely offensive April Fools Joke' as a contributor to rstat.us put it (Nichols, 2012b). As one contributor to rstat.us noted, the latter, tied as it is to the 13-year-old Creative Commons legal project (founded by the lawyer Lawrence Lessig) would "preserve the original motivation for choosing WTFPL and make the kind of statement rstat.us wants to make without alienating people' (Nichols, 2012a). While the Creative Commons 0 license would not protect derivatives of rstat.us from being made closed and proprietary, the rstat. us developers are making a political statement about software and culture-it should be totally free, and this freedom should be declared in such a way as to be recognised in as many legal contexts as possible. As Wilkinson notes, 'These systems are for the people, they should be built by the people, they give people the ability to build new things, so why not let them be entirely owned by the people?' Using a 'serious' license such as Creative Commons 0 (as opposed to the WTFPL) is part of the overall legal and political strategy of rstat.us.

Finally, I turn to activism in the legal courtroom. Elsewhere, I argue that reverse engineering exceptions to intellectual property laws have to be exercised and protected if they are to remain viable (Gehl, 2014b: 9; see also Samuelson, 2002). Without supporting the reverse engineering exception, would-be reverse engineers would watch it fade away over time. Nichols echoes this point:

I have vague hopes that [being careful about the Twitter TOS] would make it not worth Twitter's while to try and sue us, but I am not a lawyer. If they would try to sue a group including me personally, I think it would be pretty fun to go to bat over this, and I would hope organisations like the [Electronic Frontier Foundation] would help.

Nichols practically welcomes a lawsuit because she believes it would benefit rstat.us. However, she also argues that the law and technology need to be further reconciled:

I think we need more technology-aware lawyers, judges, and political representatives as more and more of our life happens on the Internet... hearing court decisions or laws and the misconceptions and conclusions they draw based on their limited knowledge makes me cringe. 
In other words, to protect reverse engineering practices, not only would the critical reverse engineers need to fight for it in court if need be, lawyers and judges will also have to be educated about new technologies. These go hand-in-hand; lawyers need precedents to study as they become educated, and technology cases need technologically-aware lawyers to argue them and set those precedents.

Ultimately, the friction caused by the legal contact point between Twitter and the alternatives revealed many complex practices and lines of thought. As should be clear, the practices of critically reverse engineering a set of functions, choosing a license (copyleft, free software, or public domain), or girding oneself against a potential lawsuit from a powerful, publicly-traded transnational firm are politically fraught. In critically reverse engineering Twitter, the alternative makers must engage in legal exegesis to see how their specific sites operate within larger contexts. In doing so, the diverging paths chosen by rstat.us, GNU social, Quitter, and Twister as they implement microblogging gives us new purchase on our legal relationship to the mainstream site Twitter.

\section{Normative}

Finally, there remains the fourth point of contact, the normative, to consider. Drawing on the literature in reverse engineering, I argue that reverse engineers do not simply take apart a technology in order to learn more about it; they do so to build something new and better than the object they are reverse engineering. This is a normative move. It might be quite instrumental; the reverse engineer might simply want to alter an object to fit an organisation's needs. Or, it might be for profit; the reverse engineer might want to make a competing product with a slightly different value proposition (as they say in business). For critical reverse engineering, however, the normative move is decidedly tied to broad (yet of course, contested) universalised goals such as freedom and equality. This is the case with the Twitter alternatives, as well. These alternatives are meant to be better microblogs, allowing their users the benefits of microblogging with more control over their data and the interface as well as more privacy from government and corporate surveillance.

When it comes to this normative point of contact between Twitter and the alternatives, a major divergence emerged during my interviews with the developers. This divergence centers on a question I put to all of them: is building a Twitter alternative activism? Some of them argued that their work is not activism, stating that the purpose of a Twitter alternative is simply to provide a politically neutral platform for their users. Others argued that the very act of coding a social media site is activism, and thus they do not see their alternative as 
neutral; rather, it is meant to shape uses and users. This is a major divergence in terms of understanding the relationship between technological development and activism. I call this a distinction between 'Platform Builders' and 'Politics Encoders.'

First, I turn to the Platform Builders. Matt Lee, Donald Robertson, and to a lesser extent Miguel Freitas argue that the Twitter alternatives they make should be neutral. For example, GNU social founder Matt Lee notes that the 'only politics I see embedded in the work is that of software freedom. I can't think of many of our users would be terribly upset about this.' Similarly, another GNU social developer, Donald Robertson, argues:

I would say that I embed ethics [rather than politics] into the software. Free software is an ethical choice to protect free expression and self-determination. Any software that is free will carry that to its users, but this does not conflict with the interests of the user. Free software is in fact built in the interest of the user. The free software community is made up of people of all different political viewpoints using the same software, so I don't believe it enforces any particular political viewpoint.

Here, both Lee and Robertson posit the neutrality of GNU social: people of all political backgrounds can use it. It does not impose a particular politics upon its user. It will not limit the end user's expression in any way.

Miguel Freitas, creator of Twister, builds on this in his discussion of the relationship between activism and technological development:

In general, I don't think technologists are good activists themselves... when activism expands much beyond the technology itself chances are it may be well off the target. I've seen bitcoin activists promoting technology as if it would solve the poverty on earth, with arguments that are based on very crude understanding of economics. I've seen Brazilian 'anonymous' group defending very incoherent positions and highly partisan and selective actions.

Freitas is suggesting that technologists ought to stick to their domains-technological development-leaving politics out of it. 
Unsurprisingly, since he has such doubts about technologists being activists, Freitas downplays the politics he might embed in Twister: 'I don't want to embed any politics into twister software except for the very specific arena it fits in, namely freedom of speech, anti-surveillance and such.' He also notes that he is careful to distinguish between political positions Twister-the software platform-might take versus his own:

If I may eventually talk about politics, let's say if I want to express an opinion about Gaza's bombing, then that will be sent from my personal account. It will be just another user's voice, not the official twister's position. I don't think the users have any problems with that. If they don't want to hear me, just 'unfollow' me. That's the standard microblogging way of doing.

Similar to Lee and Robertson, Freitas differentiates his work on Twister from what might be called 'Capital P Politics' - that is, Twister (the organisation) taking a political stance versus Freitas (the person) doing so.

How can we make sense of this? We can turn to the politics of platforms. Tarleton Gillespie (2010) argues that social media sites such as YouTube have established 'platform' as a trope, a discursive anchor providing a structural metaphor with which to understand how social media work. This term, 'platform,' is semantically rich, with multiple meanings. Gillespie draws out four meanings, noting that the architectural, computational, and figurative meanings of the term-a structure upon which to build something or present an idea-are neutral, 'typically flat, featureless and open to all' (Gillespie, 2010: 350). A fourth meaning of social media platform-the idea of a 'political platform' wherein one presents a political stance-is not neutral, but rather involves value statements. For some of the creators and developers of Twitter alternatives, their systems are akin to the first three meanings of 'platform': they are neutral, flat, and open to all. Indeed, to use these systems as a 'platform' in the political sense-that is, to build them in order to express a particular political perspective-would be abusing these systems.

This can be further explained with the ethos of the Free Software movement, which supports the Four Freedoms of software: the ability to run the program for any purpose, audit it, alter it, and distribute any changes one makes to it. On the level of property, the Four Freedoms are quite radical; as Weber (2004) notes, this perspective challenges private property as a right to exclude and instead envisions property as a right to distribute the fruits of labor. However, on the level of embedding one's politics into code in order to shape uses and thus users, the freedoms are quite conservative. In essence, the Four Freedoms focus a developer on creating a tool that is neutral vis a vis the end user's 
politics. If for some reason it constrains an end user's expression, the end user should be able to change it. This is a very libertarian, individualistic conception of technological development, one that downplays the idea that building software is activism beyond providing others with tools. The fact that these tools help mitigate surveillance and would allow users an active role in the production of the alternatives (by allowing them to modify the source code) is not interpreted as 'political' or 'activism' by these developers.

All of this reveals that Lee, Robertson, and Freitas have a particular normative relationship to Twitter that appears as they critically reverse engineer that site. Twitter is not free, which is to say that it is a platform that cannot be altered by end users. It also engages in surveillance of its users, selling access to their attention to advertisers. If it were free (which would mean it would allow users to alter its underlying code and even take that code and install on a server of their own) there would be far less need for an alternative, and Twitter's capacity as a surveillance system would be curtailed because users could modify any parts of the system that allowed for monitoring. Thus, the Platform Builder's normative move in critically reverse engineering Twitter is to supersede the Twitter platform by building a new, neutral platform that has Twitter-like features, more user control, and less centralised surveillance. Doing so would allow users to engage in microblogging in any way they wish.

In contrast, several of the Twitter alternative developers can be called 'Politics Encoders.' While the Platform Builders downplay the idea that their work is political, Politics Encoders see their work as always political, always activist. Whereas Lee, Robertson, and Freitas hesitate to claim the identity of activists, Nichols, Wilkinson, and Mannerheim readily accept it. As Nichols, an rstat.us developer, notes, 'The free software movement, in particular, is very much activism - the idea that everyone should have the freedom to read, modify, run, and redistribute software, and the people who release the code they write as such, have changed and are changing technology.' Although this sounds similar to Lee and Robertson's views, it differs in that Nichols, as a free software creator, explicitly claims the identity of activism.

Building on this, Wilkinson, also an rstat.us developer, links writing software to writing political pamphlets:

Culture, code, and activism obviously intersect. A developer's culture and identity directly influences the problems they are willing to solve, and thus their solutions, regardless of tools or technology, reflect those problems and their culture in turn. I find 'code' and 'development' here to just be evolutions 
of a type of activism that has always existed. With that in mind, yes, tech-dev is a form of activism in the same way that authoring has always been a form of activism. The usage of political pamphlets after the printing press is a similar example. It isn't new.

The distinction here is subtle but profound: whereas Platform Builders downplay the politics of their work, instead privileging the freedom users enjoy to put their platforms to whatever end they see fit, Politics Encoders interpret even the most mundane acts of coding (say, choosing a data structures or selecting a coding language) as activism, as intimately tied to and actively shaping the world around them-including uses and users. Indeed, the level of debate rstat.us developers had over the license (Nichols, 2012a) and certificate (Nichols, 2012c) reveals their intense attention to the politics of these technical decisions. Thus, in this view, building a Twitter alternative is activism on a large scale, with every design decision being political, shaping uses. Because of this, as Wilkinson argues, 'As long as writing sentences is activism, so should the writing of code, the filing of bugs, the deployment of software, the dismantling and rewriting of software, software piracy, etc.'

Hannes Mannerheim, of Quitter, extends this idea. In addition to encoding politics into technologies, he argues that technologists have to encode politics into their own lives:

I don't think it's enough to create revolutionary technology and sit back and look how your genius creation changes the world. People spend too much time writing important books and coding ingenious programs that will change how people think and too little time actually putting their politics/technology to work. It's tempting to become a 'intellectual' who aims to spread some Virus of Goodness and then lean back with a glass of wine produced by slaves, while the virus infects the moronic sheeple's minds and makes them do good instead of bad. I think you have to perform something yourself, change the way you live. Put people in the uncomfortable position of seeing stuff being done differently and on collision course with this system.

In other words, one must embody politics in oneself as one encodes it into a technology. This is quite different from the Platform Builder conception, where the platform gets built, released into the world, and then the users do with it what they will. Mannerheim argues instead for continuous, active performance of one's politics, which implies that coding, activism, and performance are always happening, always linked. 
Thus, Nichols, Wilkinson, and Mannerheim express a different normative stance towards Twitter than the Platform Builders. Whereas Lee, Robertson, and Freitas want to avoid limiting what their end users can do with their alternatives, the Politics Encoders argue that every technical choice will affect end users. As such, their actions are always fraught and require a great deal of debate and analysis. As Mannerheim notes, 'The decisions on which technology to use and which to develop [are] definitely not neutral.' In this perspective, Twitter's changes over time are viewed as having a specific political (and economic) endthat of monetising the attention of its users. As Wilkinson puts it, Twitter:

Has added promoted tweets that get injected into your timeline, promoted trending topics which defy the word 'trend' and now they are considering choosing what you see among your personally curated feed. They now want to control what you see.... They seem to want to place the right content next to the right ads.

And as Nichols notes:

I know once you get a user base the size of Twitter's, you're never going to make everyone happy, but it seems like now they're actively ignoring the needs of their users in order to serve the needs of their advertisers and shareholders.

These design decisions could be explained away by noting that despite these changes, Twitter remains a neutral 'platform' for users to express themselves in (in the first three senses Gillespie (2010) lays out), but for Politics Encoders, each of these choices is highly political, shaping what is possible in Twitter, directing users towards specific ends and thus revealing that technological change is not neutral. Likewise, a Twitter alternative should seek to ameliorate these interventions by offering other possibilities of use and communication.

Obviously, the approaches of both the Platform Builder and Politics Encoder contain implicit critiques of the other, revealing the most potent divergence among the alternatives. For the Platform Builders, embedding activist politics into the technology would limit its uses, needlessly or even destructively constraining users who want to use the tool to achieve whatever ends they see fit (including those the developers may not agree with). For the Politics Encoders, such neutrality is impossible, and thus every moment of development should be conceived of as activism; the weight then is on the developers who must think of the politics they will embed in their systems. 
This friction that occurs at the normative point of contact between Twitter and its alternatives is instructive. Should Twitter-or an alternative-be a neutral platform that does not seek to shape the content the users produce? Or is the very act of making a microblogging service activism, and when such a site fails to support progressive politics, should it be held accountable? What is productive here is that the alternatives bring this question to the fore, and instead of Twitter dictating the terms of this debate, we have multiple sites with which to think about the neutral platforms versus the politics of making social media alternatives.

\section{Conclusion}

This essay took up Anna Tsing's concept of friction-the moment when a universalised system, such as global capitalism-meets and gets a grip on a specific local context. The meeting of the global and the local is energetic; moreover, to complicate things, there need not be one 'global' meeting a local. As she argues, there are also frictions among 'multiple, contested universals' (Tsing, 2005: 89). By taking up critical reverse engineering's approach to four points of contact (pragmatic, genealogical, legal, and normative) and considering the meeting between the universalised microblog Twitter and several alternatives, I have shown other universals emerging. Specifically, the alternative makers have engaged with important questions: how closely connected to Twitter should an alternative be? How do we understand Twitter's history and the larger history of the Internet? What sort of property and distributive regimes should be deployed? What is the best course to deal with potential legal challenges? In making alternatives, are we engaging in activism or making neutral platforms? The alternative makers have principled and strong convictions about answers to these questions, leading to disagreements such as the platform builder/politics encoder distinction. However, to paraphrase Tsing, these disagreements 'are not a reason to give up on' Twitter alternatives; rather 'they are what make [the alternatives] lively' (Tsing, 2005: 88). In other words, rstat.us, Twister, and the installations of GNU social (including Quitter) are providing new ways to think about microblogging and even one of our grandest universals, communication. 


\section{Biographical Note}

Robert W. Gehl is associate professor of communication at the University of Utah. He has published research that critiques social media sites such as YouTube, Facebook, Twitter, MySpace, and blogs in New Media and Society, First Monday, Computational Culture, and Social Text. His book, Reverse Engineering Social Media (2014 Temple UP), explores the architecture and political economy of social media.

\section{Notes}

[1] GNU social is a free software project to build microblogging software that can be installed on one's own server. This can then be federated, which means that users can sign up for one instance of GNU social and follow each across instances of GNU social installed on servers around the world. GNU social instances include Indy.im, Gnusocial.no, LoadAverage.org, and Quitter.se, so a registrant at Indy.im can follow one at LoadAverage. org and vice-versa (see Ortega 2015 for more about federation). The specific instance Quitter.se was profiled in this paper because it features an interface, Qvitter, that closely mimics Twitter's interface in ways that a standard installation of GNU social does not.

[2] All interviews were conducted via email or in person between May 2014 and February 2015. They were preceded with the University of Utah's Institutional Review Board consent notices. Interviews took place over several days with multiple follow-up sessions. I would like to express my sincere thanks to the interviewees, who deeply educated and challenged my understanding of making alternative social media.

[3] Twitter's Developer Agreement and Policy (https://dev.Twitter.com/overview/terms/ agreement-and-policy, last accessed 2 November 2014) specifically prohibits reverse engineering: 'You will not or attempt to (and will not allow others to)... reverse engineer, decompile, disassemble or translate the Twitter API, or otherwise attempt to derive source code, trade secrets or know-how in or underlying any Twitter API or any portion thereof.' The legality of such a clause is disputable (Samuelson and Scotchmer 2002). It is likely there to constrain third party developers who seek to build applications on top of Twitter's platform. 


\section{References}

Andrejevic, Mark. 'Surveillance in the Digital Enclosure', The Communication Review 10 (2007): 295-317.

Atton, Chris. Alternative Media (London: SAGE, 2002).

Boyle, James. 'The Second Enclosure Movement and the Construction of the Public Domain', Law and Contemporary Problems 66.33 (2003): 33-74.

Bratich, Jack. 'User-Generated Discontent', Cultural Studies 25 (September 2011): 621-40.

Chikofsky, Elliot and James H. Cross. 'Reverse Engineering and Design Recovery: A Taxonomy', Software, IEEE 7.1 (1990): 13-17.

Chun, Wendy Hui Kyong. Programmed Visions: Software and Memory (Cambridge, Mass.: MIT Press, 2011).

Coleman, Gabriella. 'Code Is Speech: Legal Tinkering, Expertise, and Protest Among Free and Open Source Software Developers', Cultural Anthropology 24 (August, 2009): 420-54.

Couldry, Nick and James Curran, eds. Contesting Media Power: Alternative Media in a Networked World (Oxford, UK: Rowman \& Littlefield Publishers, 2003).

Eilam, Eldad. Reversing: Secrets of Reverse Engineering (New York, NY: Wiley, 2011).

Feenberg, Andrew. Lukács, Marx, and the Sources of Critical Theory (New York: Oxford University Press, 1986).

Foucault, Michel. 'Nietzsche, Genealogy, History', in Paul Rabinow (ed.). The Foucault Reader (New York: Pantheon Books, 1984), 76-100.

Fuller, Matthew. Software Studies: A Lexicon (Cambridge, Mass.: MIT Press, 2008).

Gehl, Robert W. Reverse Engineering Social Media. (Philadelphia, PA: Temple Press, 2014a).

Gehl, Robert W. 'Critical Reverse Engineering: The Case of Twitter and Talkopen'. SSRN Scholarly Paper ID 2470750 (Rochester, NY: Social Science Research Network, 2014b) http://papers.ssrn.com/abstract=2470750

Gillespie, Tarleton. 'The Politics of 'Platforms", New Media \& Society 12.3**(2010): 1-18. Ingle, Kathryn A. Reverse Engineering (New York: McGraw-Hill, 1994).

jpfox. 'Possible to Add a Dedicated Url Field?' GitHub (14 January, 2014) https://github.com/ miguelfreitas/twister-core/issues/63

Kitchin, Rob and Martin Dodge. Code/space: Software and Everyday Life (Cambridge, Mass.: MIT Press, 2011). 
Koopman, Colin. Genealogy as Critique: Foucault and the Problems of Modernity (Bloomington, IN: Indiana University Press, 2013).

Mackenzie, Adrian. Cutting Code: Software and Sociality (New York: Peter Lang, 2006).

Nichols, Carol. 'Future Direction of Rstat.us Development'. Email Archive. Librelist Archives (7 January, 2012a) http://librelist.com/browser//rstatus/2012/1/7/future-direction-of-rstat-usdevelopment/

Nichols, Carol. 'Changing the License to CCO \#443'. Repository. GitHub (11 January, 2012b) https://github.com/hotsh/rstat.us/pull/443

Nichols, Carol. 'Certificate Authority'. Mailing list archive. Librelist Archives (10 July, 2012c) http://librelist.com/browser//rstatus/2012/7/10/certificate-authority/

Ortega, Manuel. 'GNU Social: Federation against the Social Model of Twitter'. Las Indias in English, (April 8, 2015) http://english.lasindias.com/gnu-social-federation-against-the-socialmodel-Twitter

Rodriguez, Clemencia. Fissures in the Mediascape: An International Study of Citizens' Media (New Jersey: Hampton Press, 2001).

Samuelson, Pamela. 'Reverse Engineering under Siege'. Communications of the ACM 45.10 (2002): 15-20.

Samuelson, Pamela and Suzanne Scotchmer. 'The Law and Economics of Reverse Engineering'. The Yale Law Journal 111.7**(2002): 1575.

Siles, Ignacio. 'From Online Filter to Web Format: Articulating Materiality and Meaning in the Early History of Blogs'. Social Studies of Science 41 (September, 2011): 737-58.

Sippey, Michael. 'Changes Coming in Version 1.1 of the Twitter API'. Twitter Blogs. (16 August, 2012) https://blog.Twitter.com/2012/changes-coming-to-Twitter-api

Terranova, Tiziana. 'Free Labor: Producing Culture for the Digital Economy'. Social Text 18.2 (2000): 33-58.

Tsing, Anna Lowenhaupt. Friction: An Ethnography of Global Connection (Princeton, N.J.: Princeton University Press, 2005).

Weber, Steven. The Success of Open Source (Cambridge, Mass: Harvard University Press, 2004). 


\section{OPEN HUMANITIES PRESS}

The Fibreculture Journal is published by The Fibreculture Journal Incorporated in partnership with Open Humanities Press. 
issue 26: Entanglements - Activish and dech pology

\title{
FCJ-191 Mirroring the Videos of Anonymous: Cloud Activism, Living Networks, and Political Mimesis.
}

Adam Fish

Lancaster University

\begin{abstract}
:
Mirrors describe the multiplication of data across a network. In this article, I examine the politics of mirroring as practiced on videos by the hacktivist network Anonymous. Mirrors are designed to retain visibility on social media platforms and motivate viewers towards activism. They emerge from a particular social structure and propagate a specific symbolic system. Furthermore, mirrors are not exact replicas nor postmodern representations. Rather, mirroring maps a contestation over visibility that entangles both cloud activists and platform firms.
\end{abstract}

doi: $10.15307 / f c j .26 .191 .2015$

\section{Introduction}

In August 2012, Wikileaks was hit with a massive Distributed Denial of Service (DDoS) attack from a mysterious group appropriately titled Antileaks. DDoS assaults occur when multiple 
computers simultaneously 'refresh' a website causing it to overload and shutdown. A shaken-up Wikileaks tweeted: 'The range of IPs used is huge. Whoever is running it controls thousands of machines or is able to simulate them' (Kerr, 2012). This is a constant problem for Wikileaks. Their website reads: 'WikiLeaks is currently under heavy attack. In order to fully protect the CableGate archives, we ask you to mirror it again' (Wikileaks. org). Eventually repelling the Antileaks attack, Wikileaks again took to Twitter to boast: 'DDoS proof, financially \& geographically diverse. We're ready to rumble' (Kerr, 2012). For Wikileaks, mirroring means copying and pasting the CableGate archives in resilient servers so that the content remains visible.

Infamous for Guy Fawkes masks, battles with Scientology and PayPal, support for Wikileaks and Arab Spring activists, hacks of HBGary and Stratfor, braggadocios videos, Lulzsec, and 'Internet freedom,' Anonymous has developed a way for their videos to stay visible on YouTube. The practice they call 'mirroring' is performed when another Anonymous account downloads and then re-uploads the content on YouTube. For the enemies of Anonymous, videos mirrored on different YouTube accounts are difficult to monitor, and if possible, censor. This article is about the politics of data mirrors, what they are and what they do. Below, I introduce mirroring as a political and aesthetic practice similar to but distinct from copying. After detailing my methods which included participating in video mirroring and interviewing Anonymous video producers, I explain what mirrors are, what they are designed to accomplish, and from what social form they emerge.

Mirrors are not merely replicas. They map a contestation over visibility, seek to inspire activism through a process of political mimesis (Gaines, 1999), and evince the complex entanglements of cloud corporations and cloud activists-political actors dependent upon cloud platforms such as corporate social media. I conclude this essay by explicating how the video mirrors of Anonymous emerge from the social form of the living network (Wiedemann, 2014) and are executed to propagate the shared symbolism of an improper name (Deseriis, 2012). In summation, I define mirroring as an emergent practice of a freedom of information movement (Beyer, 2014). As mirroring is an understudied phenomenon practiced across a range of institutions and groups, it was critical to synthesize a wide-range of literature from critical approaches to Anonymous (Deseriis, 2012; Wiedemann, 2014), sociology (Couldry, 2010), feminism (Barad, 2003), and media studies (Askanius, 2013; Gaines, 1999). In the section that follows, I explain the research methods used and provide a brief introduction to Anonymous. 


\section{Methodology and Background}

This article reports on seven years of ongoing research into the video production and dissemination practices of Anonymous. Using YouTube, Twitter, IRC chatrooms, Anonymous websites, and mainstream media, I have tracked Anonymous since 2008 when they first gained public attention because of their offline and online protests against the Church of Scientology. During 2010's Operation Payback, a protest against opponents of piracy and Wikileaks, I began watching hundreds of YouTube videos of all genres, lengths, and technical skill. During their 2011 actions in support of Arab Spring activists, I used my YouTube account to mirror videos produced by Anonymous and thereby gain participatory experiences. As such, this research is an example of politically-engaged 'militant ethnography' in the virtual sphere (Juris, 2007).

By closely following Anonymous on YouTube, I identified the most active producers in terms of output, views, and practical skill. I developed a list of 80 Anonymous YouTube producers to which I sent a questionnaire via YouTube's private user-to-user messaging service. The open-ended questions solicited responses about motivation, inspiration, production practices, and whether or not they mirrored videos. Of the 80 to which I wrote, 14 responded, many in detail. I conducted intensive follow-up interviews with four of these respondents. With the goal of connecting media production practices to reflexive discourse, or what Caldwell (2008) calls, 'industrial reflexivity,' I focus considerable attention on the video oeuvres of interviewees.

Interviewees include THXi330 who has made seven videos that have been viewed over 283,500 times and TheAnonMessage who produced 88 videos with 9,083,428 views. To put this into context, another informant, TheAnonShade, produced 10 videos and slightly more than 2,500 views. Anonymousworldwar3 has made 31 videos with 662,466 views. Alucard 9692010 has made 274 videos with 857,463 views. These are not collective channels but rather single user accounts. The project participants are indicative of the small population of highly competent Anonymous YouTube producers capable of visually evocative and politically aware uses of YouTube. From the differing metrics, it is clear that diverse Anonymous mirror accounts have distinct impacts. Through the course of the research, I encountered YouTube accounts mirroring Anonymous videos along with other less political videos in such a manner that suggests that they were mirroring videos not for the politics but in order to generate advertising revenue. In this article, I focus exclusively on the politically motivated video producers and mirrors, some of whom criticized those mirroring Anonymous videos for profit. Many fractions have emerged within Anonymous with different understandings of what the group is and does. I focus on a small representative of the Anonymous video producers who make and mirror videos on YouTube. 
Since they emerged from the uncensored messageboard 4chan in 2003 , Anons, as they are called, have carried out a range of activities. While not many of those associated with Anonymous are politically motivated, this article focuses on those who participate in Anonymous as a form of political activism. An example of their multifaceted hacktivism was Operation Last Resort in 2013. The operation was initiated to avenge the suicide of freedom of information activist Aaron Swartz (described below), which Anonymous attributed to over-zealous enforcement of outdated computer crime laws. Like all major Anonymous operations, Operation Last Resort was a visual spectacle which included the hijacking of an MIT website to make a Swartz tribute, the production and mirroring of videos, the hacking and releasing of the names and contact information for 4000 banking executives, and the usurping of the U.S. Sentencing Commission website (Blue, 2013). In these actions Anonymous are simultaneously 'part human rights technology activism and part performance spectacle' (Coleman, 2012a: np). While multifaceted, at times paradoxical and always elusive, some insights about Anonymous can be gathered from connecting mirroring practices to social forms and the circulation of symbols. First, however it is important to critically discuss mirrors, what they are, and what they are designed to do.

\section{What Are Mirrors?}

Mirroring is not only for activists. It is also an important practice which enables cloud computing companies to synchronise data in data centres around the world. Microsoft, which provides a number of cloud computing services, defines 'database mirroring' as the maintaining of 'two copies of a single database that must reside on different server instances' (Microsoft, 2014). Microsoft and other companies with immense investments in server farms-Amazon, Google, and Facebook, to name but a few high profile examplesneed to offer robust, secure, and non-delayed access to content. To do this, they strategically locate multiple copies of the same data. Activists also mirror data. Today, sites in eleven European nations offer the Wikileaks mirror (Wikileaks.info, nd). The largest Bittorrent-based peer-to-peer file sharing service in the world, the Pirate Bay, mirrors links to servers located in countries where their service is not yet illegal. Sites like baymirror. com and piratebaymirror.com, enable users to route around legal blockage of root access to the Pirate Bay. For similar goals but on a smaller scale, Anonymous activists download YouTube videos produced by other Anonymous producers and re-upload these same videos on different accounts. Not unlike the mirroring practices of Wikileaks, the Pirate Bay, and Microsoft, this form of mirroring is executed in order to preserve data against politically motivated erasure.

Mirroring has received little focused scholarly attention in critical media studies. Texts like Mirror Worlds (Gelernter, 1992) used the mirror as a metaphor to describe the parallel and 
immersive world that would be created by networked computers. Years before Second Life, and Oculus Rift, Mirror Worlds claimed that virtual worlds were actual places. Today, information companies and information freedom activists alike call data duplication mirroring but often fail to acknowledge how the symbolism of this term may impact its use. Mirrors are more complex entities than simple facsimiles. With multiplication comes the diversification of potential cultural impacts. 'Copying is not merely reproducing the same as discreet objects, but coding cultural products into discreet data and communicating such coded copies across networks: seeding and culturing,' Parikka states (2008: 76). As cultural processes, mirrors echo the intricacies and limitations of data practice. I endeavor to explain how for information activists and information firms, mirroring is an exploit of networks and computers to remain visible through replication.

While mirroring has sustained little attention, copying has received far more, as it relates to major cultural and financial issues such as piracy and precedent setting court cases involving the likes of Napster, Google, Viacom, and the Pirate Bay (Lindgren and Linde, 2012; Postigo, 2012). Copying has largely been framed by academics in terms of a battle between media corporations and the 'free culture' of file sharing. Lessig (2004) has argued that by criminalizing copying, copyright laws dampen cultural innovation surrounding sharing. The political potential of copying can be traced back to the Gutenberg press and the duplication of Lutherian theses, East German facsimiles, and Xeroxes of the Pentagon Papers. Gitelman (2011) emphasizes not the leaking but the photocopying of the Pentagon Papers by whistleblower Daniel Ellsberg as a politicized act of duplication. The unfortunate case of Aaron Swartz, a free culture activist caught downloading thousands of academic journal articles, which he intended to redistribute through mirrored databases, who took his own life in 2013 amidst a prosecution, speaks to the fervor surrounding today's copy culture.

The politics of copying brings us to the early days of free and open source software (FOSS). Millions of computer operating systems run on FOSS such as GNU/Linux which was created by volunteer coders copying and sharing source code (Kelty, 2008). For the FOSS community, copying is a technical practice, an instigator for community, and a moral imperative (Coleman, 2012b). Anonymous and the Pirate Bay share with the FOSS community a belief that copying is practically necessary, socially integral, and an inalienable right (Halliday and Arthur, 2010). Co-founder of the Pirate Bay, Peter Sunde, believes that we are genetically coded to copy: 'People learn by copying others. All the knowledge we have today, and all success is based on this simple fact - we are copies' (cited in Ernesto, 2014). For the Pirate Bay, mirroring is necessary to secure the visibility and accessibility of their file-sharing service. Video mirroring is an activist labor that seeks to overcome or bypass copyright regimes and censorship. Beyer (2014) sees in Anonymous, Wikileaks, and the Pirate Party an emergent 'freedom of information movement.' Mirroring is a little understood practice of this movement. 
Video mirroring is both practical and aesthetic. From the Dada cut-up techniques of the 1920 s to punk rockers in the 1970s resignifying the safety pin, copying, pasting, and recontextualizing have long been important sources of aesthetic creativity. Today's video activist is also a bricoleur who usurps and remixes found sound and footage. Manovich (2001) makes clear how the aesthetics of software recontextualize the past. For example, Navas (2010) describes Apple's original graphic user interface as a copy of the IBM command line remixed with the metaphor of a 'desktop.' He describes 'regenerative remixes' (Navas, 2010) which are social media sites like Tumblr that use interoperable APIs to copy and remix content into ever-changing, real-time, personalized sites. Like regenerative remixes, mirrors result from the copy/paste functionality of computers, networks, and social media platforms. Unlike video and API mashups, however, mirrors are icons that are aesthetically similar to source material instead of being unique iconoclasms.

The political power of mirrors is achieved through bestowal, homogeneity, and ubiquity while the politics of remix is achieved through appropriation, heterogeneity, and novelty. Mirrors are centrifugal, their constitution requires 'seeding' (Parikka, 2008: 76). Remix is centripetal, produced through a mode of gathering (McKelvey, 2014). Video mirrors of Anonymous are different from peer-to-peer file sharing for the simple reason that the shared files reside in proprietary cloud servers and not on private personal hard drives. The sharing of video mirrors happen within a single cloud system while peer-to-peer sharing transects proprietary, public, and private platforms. In this manner, Anonymous video producers are dependent upon corporate cloud platforms such as YouTube and Google.

A common belief is that mirrors are exact replications, merely displaced within databases. A more complex social constructivist perspective sees mirrors as symbolic representations. Barad (2003) challenges both 'naïve realist' as well as constructivist interpretations of scientific knowledge. She offers a third interpretation of mirrors when she says '...the representationalist belief in the power of words to mirror preexisting phenomena is the metaphysical substrate that supports social constructivist, as well as traditional realist, beliefs' (Barad, 2003: 802). Mirrors are neither realist copies nor constructed depictions. They offer viewers another perspective on a practice of visual contestation. Mirrors are performances enacted within and between relational databases with the goal of securing video power.

Mirrors can be read as constitutive material practices that point to frictions and paradoxes of what can be called cloud activism. This interpretation of mirrors requires a shift from reflection to diffraction that "does not produce "the same" displaced, as reflection and refraction do. Diffraction is a mapping of interference, not of replication, reflection, or 
reproduction. A diffraction pattern does not map where differences appear but rather maps where the effects of differences appear' (Haraway, 1992: 300). Anonymous video mirrors are diffractions that visually map a contestation over networked visibility.

Video mirroring is but one practice of visibility indigenous to a particular class of cloud activism. This is a preliminary attempt at defining one small iteration of mirroring that may link in practice to other forms of politicized copying exercised by the freedom of information movement (Coleman, 2014; Beyer, 2014). Below, I critically analyse mirrors, what they do, as well as the social formations from which they come.

\section{What Do Mirrors Do?}

The mirrors of Anonymous attempt to do two things: 1) proliferate and preserve videos so that they might 2 ) motivate others to mimic the radical personae represented in the videos.

\section{Mirrors as a Will to Visibility}

Video mirroring can be theorised in terms of a will to visibility. The term 'will to visibility' refers to both the desire and the capacity to be seen. It denotes the 'intrinsic, rational, non-empirical ground of social process' that accounts for both the 'strategies' as well as the 'conditions of visibility' (Criado, 1995: 197). Mirrors map a will to visibility not unlike the act of voicing. Couldry (2010: 7-10) defines the act of giving voice as a process that is socially grounded, reflexive, agential, embodied, material, and valuable beyond market fundamentalism. Anonymous videos are a visualisation of voice. This will to visibility is contested by the 'scopic regimes' of Western technoscience and statecraft that reserve the power to make something visible, legible, and able to be controlled (Haraway, 1997; Jay, 1992; Scott, 1999). Historically, writing and printing prioritised the ocular and thus gave power to those who could read, write, and print, and therefore cast mortal judgments based on ledger knowledge (McLuhan, 1964; Ong, 1977). Thus, visibility 'lies at the intersection of the two domains of aesthetics (relations of perception) and politics (relations of power)' (Brighenti, 2007: 324). In short, power is enacted through the capacity to make things visible. Mirroring retains visibility for activist video while also making evident the entanglements of cloud activists and information corporations. Mirrors represent a proliferation of content to avoid erasure, maximising the potential for political recruitment. 
Anonymous as a political project began in 2008 when a video was leaked on YouTube of Tom Cruise energetically celebrating Scientology. Scientology initiated a comprehensive campaign to have the video deleted from YouTube. Lawyers for Scientology claimed the video infringed upon their copyright (Denton, 2008). According to my informants, Scientologists were instructed to 'flag' this video as inappropriate before it was rapidly mirrored across YouTube. Flagging refers to the option provided by YouTube to users to notify YouTube that they consider the content offensive or copyrighted. 'Flags,' according to Crawford and Gillespie (2014: 3), 'act as a mechanism to elicit and distribute user labor-users as a volunteer corps of regulators.' Reducing online commentary to simplistic binaries of "offensive" versus "inoffensive," flags potentially diminish the generative potential of agonistic debate over what has the right to remain visible. An unknown quantity or frequency of flags and the video is automatically removed until the user petitions to have it reinstated. To avoid this, dedicated mirror channels exist to insure that flagging actions are distributed across a number of mirrors making it difficult to censor all Anonymous videos. This was the beginning of the Anonymous war against Scientology, Project Chanology, which was announced through a heavily mirrored YouTube video, Message to Scientology (ChurchOfScientology, 2008). In this and subsequent operations, video mirroring played a crucial role in Anonymous securing visibility. Mirroring is a diffraction; it creates a militant topology, or a mapping of conflict.

A second video followed from YouTube user ChurchOfScientology, Call to Action, sought to combat mainstream media misrepresentation of Anonymous as 'superhackers' and not a 'collective...from all walks of life' (ChurchOfScientology, 2008). Call to Action concluded by inviting viewers to protest outside of Scientology centres on February 10th, 2008. Because of what some Anons consider to be another politically motivated flagging initiative, Call to Action was taken down by YouTube, which cited a violation of 'terms of service.' Soon after, however, the video was mirrored across YouTube. In operations linked to the video, Anonymous began DDoS confrontations, prank calling, and sending black faxes and unpaid pizza to Scientology headquarters. In these ways, video was the means for political mobilisation. The impact of the videos would have been minimised were it not for mirrors.

Code of Conduct (ChurchOfScientology, 2008) was a third video of Project Chanology. Where Message to Scientology was addressed to Scientology and Call to Action to the mainstream media, Code of Conduct was a public document for other Anons citing 22 principles to follow for successful physical protests. Code of Conduct was vigorously mirrored. Anonymousworldwar3 explained to me why they mirrored Code of Conduct: 'at that time we were organising real life protests and I felt it was important to get that video/ info out there before going out into the "real world". Despite the flagging that Anonymous assumed was resulting from Scientology, all three of these videos remained visible because of the practice of mirroring. 
According to user AnonymousFacts, Scientology forced YouTube to delete the account and several others that were instrumental in making and releasing these anti-Scientology videos (AnonymousFacts). In the video, A Call for Video Mirroring, Anonymousworldwar3 requested viewers to download and re-upload their Arab Spring videos. The narration states:

I noticed someone started flagging these video's as inappropriate and/or voting them down systematically. Apparently, not all agree to the right of free speech... As much as I regret this, there is nothing I can do to prevent this. That is why I want to ask all of you to mirror these video's before it is too late (Anonymousworldwar3, 2010).

In the comments, user @CavalierPosts asks how to mirror and Anonymousworldwar3 responds with instructions. For Anonymousworldwar3 being a centralised hub for videos made Anonymous vulnerable while decentralisation through mirroring distributed risks of visual erasure across the network. Dependent as cloud activists are on corporate platforms, the highly mobile practice of mirroring, which distributed risk across the platform, made Anonymous less vulnerable. Mirroring is a way Anonymous resisted Scientology's counterattack. Interviewee Alucard 9692010 agreed:

Back in 2008 when Anonymous started its war against the Cult of Scientology, a very large quantity of Anonymous videos were created to educate and expose Scientology to the general public. But then I (and many others) noticed that Scientology started a false flagging campaign and took down quite a few of these anonymous videos. So, as a response, Anonymous everywhere (me included) started to re-upload the removed videos everywhere. Nobody likes a cheater :). They took one of our videos down, we uploaded it 10 times again on 10 different channels. Even people that had nothing to do with Anonymous started to upload our videos on their channels when they noticed the Scientology false flagging campaign. This is how this channel has started. Since then it evolved, uploading mirrors of videos in chronological order following certain stories as they happened (ACTA, occupy movement, the Arab spring and so on). A anonymous information channel.

Both Scientology with their alleged flagging campaign and Anonymous with their mirroring practices, exploited the affordances provided by YouTube. Ostensibly a way for YouTube users to help govern the site (Crawford and Gillespie, 2014), flagging is inherently political. The political application of mirroring too evolved out of YouTube's desire for scale and growth by not limiting user-account registrations to specific Internet protocol addresses. 
In this way, both Scientology and Anonymous exploited an affordance as a weapon in a semiotic war. As I will describe in full below, this entanglement is indicative of cloud activism.

Video mirroring is a practice that exploits the scalability of social media platforms to maximise visibility and resist erasure through flagging. Unlike video production, mirroring does not require cinematographic or editing skills but rather competencies in downloading, uploading, and user account management. For example, TheAnonShade focuses exclusively on mirroring videos about Anonymous operations in the Middle East and Asia. They describe their activities:

I am a messenger of the Shadowy part of Anonymous, I just mirror videos that NEED and have the itching to get out. My role is the other side of the world; which is Syria, Bahrain, India, and China. ... I, as well as my team have access to one of the biggest Anons. So when they are about to upload a video immensely important to the collective. We are the first people to do a first reupload to their specific video.

As mirroring is a mapping of a displacement so too are mirroring actors geographically dispersed. Anonymous videos are incorporated into operations against the politically powerful such as Syrian President Bashar Al-Assad and organisations such as Scientology, the NSA, and PayPal with the economic resources to retaliate against antagonists. Mirrors utilise the replicability of digital information, the distributed nature of networks, and the pseudo-anonymity provided by some social media platforms to create an innovative form of visibility. As THXi330 said, mirrors enable Anonymous to explore the "possibilities of viralisation.' Mirroring is one experiment with viralisation that exploits pseudo-anonymity, replicability, and networks. Mirroring is both an offensive manoeuvre to propagate symbols and circulate affect and a defensive manoeuvre to remain visible in a contested and privatised cloud-based public sphere. Remaining visible is the goal on the infrastructural level while political mimesis, the hailing of bodies to feel and move like the revolutionary beings depicted on video screens, is the aim on the subjective level.

\section{Mirrors as Political Mimesis}

Because of their verisimilitude, mirrors are central metaphors with which to frame representation, identity, and symbolism. One contribution of postmodernism is 
constructionism. A reaction to realism, constructionism is relativistic in its interpretation of representations. Taussig (1980) interprets rituals as the personification of icons and as invitations for others to embody the personified icons. These mimetic rituals are often augmented by masks that temporarily transform initiates into liminal states of shared pseudonymity. Gaines (1999) blends the work of Taussig and Williams (1991) to develop a theory of 'political mimesis,' or how documentaries seek to move the bodies of audiences. Drawing from Williams (1991) and her typology of bodily reactions to horror (scream), melodrama (cry), and pornography ('come'), Gaines (1999) describes a fourth genre, the political documentary, which is designed to motivate viewers to take up political action. Gaines (1999: 93) differentiates mimesis from mimicry that carries 'connotations of naïve realism, mindless imitation, mechanical copying, and even animality'. Anonymous represents a politically engaged, networked form of video production and masked activism that calls upon supporters to mirror videos and copy revolutionary bodies. This type of mimesis is not "naïve realism," rather it is a form of corporeality more immediate than second-order representation. The Guy Fawkes mask enables participants to mimetically embody a radical affect. It provides high visibility and shared symbolism while retaining pseudonymity on corporate social media.

Anonymous YouTube producers are examples of what Anonymous calls 'moral fags.' With their emergence from the shady underworld of the imageboard 4chan, Anonymous uses this crude language to describe politically active Anons as opposed to those who are just in it for fun, trolling, or the 'lulz'. Moral fags produce politically motivated videos that attempt to 'pump up' supporters and bring 'melancholy' to their enemies as THXi330 and TheAnonMessage informed me. One informant, Anon Pyrriel, self-identified as a moral fag:

In other words I am with the part of Anonymous that fights for all that is unjust in our opinion. I am not affiliated with the part of Anonymous that posts flashing pictures on epilepsy websites and tries to 'troll' the Internet.

Worldunitedanonymous explains his motivation for posting political videos in this way:

Videos calling for peace and justice impact me the most. For example during Oplsrael several videos surfaced calling for peace in the region, even when both sides had things to be angry at the other about. I find stuff like that really inspirational because it shows that humanity can come together in the name of peace even when no sign of peace is in sight. 
Anonymousworldwar3 uses 'imagery of protest because I believe these images have the ability to inspire people to get out there and protest themselves, while it also gives the videos a certain epic quality and stimulates a feeling of agency in the viewer.' Worldunitedanonymous reiterated that 'moral fags' produce videos to bring about world peace and end greed, corruption, and exploitation. Alucard 9692010 said 'moral fags' agitate to defend freedom of information, free speech, and an unregulated Internet. These videos can be seen as 'radical media' (Downing, 2001) or 'mobilization videos' (Askanius, 2013)-forms of communicative action that attempt to galvanise support for political operations.

Downing (2001) describes radical media as content that invites audiences to participate in social movements and media production. Radical media enters into paradoxical relationships with corporately owned Internet platforms such as YouTube. In the case of Anonymous, 'radical' is a preferable term to 'alternative,' 'participatory,' or 'independent' which are either hegemonic in their complicity with dominant media systems and corporations or counter-hegemonic in their attempts to reform hegemonic systems. Radical media is revolutionary and anti-hegemonic, seeking transformation of the present system. Anonymous is a radical movement in its no compromise rejection of states' rights to surveillance and their extreme views on free speech maximalism and 'radical transparency' (Sifry, 2011). Yet while their perspective on the Internet as an information commons can be decisively anti-corporate and post-capitalist, the paradox is that radical media often use corporate and increasingly centralised media platforms such as YouTube to perform their radical will to visibility.

According to DCHTID247, the goal of political mimesis is 'to mirror to the world that there is non-hierarchical groups out there that are fighting for freedom.' Anonymousworldwar3 maintained: '...anon is just a symptom of this new structure imposing itself on today's world: it is one of the first glimpses of a "new world" that unfolds itself on the basis of this new communication and information-infrastructure and the principles that organise this.' In this way, Anonymous videos are 'mobilisation videos' which do not seek to re-affirm social movement solidarity but rather seek to galvanise adherents in a particular cause (Gregory et al., 2005). Because they attempt to energise those already converted, mobilisation videos tend to be reductive and fundamentalistic. Askanius (2013) categorises political mash-up videos as examples of mobilisation videos with three unique qualities: aesthetic generativity, genre hybridity, and production democratisation. Anonymous videos are an example of the 'coalition model' of radical media wherein the goal is not a finalised film or fame for the producer, but rather the initiation of a political process (Christiansen, 2009). 
For those willing to talk, either rationally or passionately, the Internet can constitute the conditions for a public sphere (Habermas, 1987), a digital public sphere (Dahlgren, 2005), a subaltern counterpublic (Fraser, 1990), or an agonistic and passionate public sphere (Mouffe, 2005). While YouTube has proven to be an important site for political debates, mobilisation videos like those made by Anonymous are clearly not designed to simulate a version of a public sphere. In the case of Anonymous, viewers leave comments but producers rarely comment back to viewers. The majority of the commentary on Anonymous videos consists of declarations of support or troll-like denouncements. This is video as a direct will to visibility. It is propaganda to mobilise supporters, not media to provoke reasoned, agonistic, or subaltern debate. In short, Anonymous videos magnify a collective affect and YouTube is the platform for this shared feeling.

On one level, Anonymous mirrors are representations. They are an invitation to viewers to enact political mimesis by modeling themselves after masked revolutionary bodies depicted on screen. On another level, by not pointing to an ontogenetic moment but rather being an ongoing struggle, Anonymous mirrors are a form of praxis transfigured by conflict, affectually shared, and existing in a paradoxical relationship to information firms. Either as political mimesis or practice, Anonymous videos represent sophisticated adaptations to network affordances in a pursuit of visibility.

Mirroring is a practice that emerges from a particular social structure designed to propagate a specific set of symbols. The terms 'cloud activism,' 'living networks,' and 'improper names' may assist us in understanding how mirrors emerge from the structural and symbolic systems of Anonymous

\section{Structures and Symbols of Anonymous}

Anonymous is an example of 'cloud activism' entangled in paradoxical relationships with for-profit social media firms (Milan, 2013; Nielsen, 2009). Bringing to the theory of cloud activism the concept of the 'living network' adds a missing dimension of the importance of affect in the formulation of Anonymous (Stoehrel and Lindgren, 2014; Thacker, 2004; Wiedemann, 2014). I add the notion of collective pseudonyms or 'improper names' in order to discuss the important role played by symbols in the growth of Anonymous (Deseriis, 2013). Mirroring is an activist practice particular to a structure of feeling and symbolism requiring the synthesis of these theories to explain. 


\section{Cloud Activism}

Utilising geographically dispersed servers, cloud computing allows users to access remote data and applications. 'Cloud activism' is 'activism based on a structure that the user does not control or own the way they would an individual centralised personal computer' (Nielsen, 2009: 279). Cloud activism is activism that is dependent on privatised information infrastructure. As a form of activism performed on freely accessible and privately held social media platforms such as YouTube, mirroring is an example of cloud activism entwined with servers and databases held in private.

Based on her ethnographic research into the relationships between environmentalists, indigenous people, and illegal loggers in the rainforests of Indonesia, cultural anthropologist Anna Tsing identified that the places these actors meet constitute a 'zone of awkward engagement' that is characterised by friction or the 'awkward, unequal, unstable, and creative qualities of interconnections across difference' (Tsing, 2005: 4). These interconnections 'remind us that heterogeneous and unequal encounters can lead to new arrangements of culture and power' (Tsing, 2005: 6). For Anonymous, YouTube is a 'zone of awkward engagement' wherein their competencies with software, social media, and video permit a small and awkward shifting of video power. The mirroring practices of Anonymous are a form of cloud activism that exposes the imbroglio of information activists using profitseeking social media platforms. As Gillespie (2010) articulated, the semiotics of 'platforms' is intentionally left open so as to remain versatile. Through mirroring, Anonymous exploits not just the semiotic but also the practical variability of social media platforms.

User Anonymousworldwar3 describes its entanglement with cloud-based social media firm YouTube:

At a certain point in time, this was (Anonymousworldwar3) the largest anon channel on YouTube, which I think was not a great thing for a number of reasons: it made anon's presence on youtube quite vulnerable, since it allowed people to take just one channel down and most of anon would be wiped from youtube + it gave me a far too central role within anon, which goes against the concept of decentralization within anon itself. So we started to make sure all of the anon videos were mirrored on a number of youtube channels + where at first we were only with two or three people doing videos, we started some sort of 'video-training' to make sure there would be a whole lot more people making videos, both for the sake of being able to hide within a larger 
crowd, and to make anon's youtube presence less centralized and thus less vulnerable.'

For Anonymous and their targets such as the U.S. Sentencing Commission in 2013's Operation Last Resort, YouTube constitutes a 'zone of awkward engagement.' The asymmetries in this interdependence may result in a temporary shifting of the power of visibility. This visibility is partly enabled by knowing how the platform works: it's rules, expectations, and weaknesses. Anonymous is a living network that understands how to (mis)use cloud-based social media platforms to generate and circulate affect.

\section{Living Networks}

While describing the relation of Anonymous to proprietary social media platforms, cloud activism does not explain the affect that links activists in circulations. Gregg and Seigworth (2010: 1) define affect as the visceral forces that 'drive us toward movement, toward thought and extension.' Hansen (2004) considers affect a key aspect in the process of engagement with digital information (see also Featherstone, 2010). Similarly, Lazzarato treats digital technologies as 'motors of affective energy' (2007: 115). It is the combined emotional intensity and behaviour that binds individuals within Anonymous, not collective 'identities, myths, or narratives' (Wiedemann, 2014: 6). Anonymous is a 'living network' that is 'inherently dynamic, undergoing constant and variable changes, both within the composition of individual nodes, and in the relations between nodes' (Thacker, 2004: np). In emphasising movement and historical change and de-emphasising static nodes, Thacker underscores not 'network effects' but 'network affects' (Thacker, 2004: np). As Stroehel and Lindgren (2014) identify, the affective intensities of Anonymous drive their political projects. Anonymous as a variegated 'living network' can be observed not only through mirroring but also through their DDoS assaults.

Mirroring, along with DDoSing, is a tactic that one does not need to be a hacker to do. While the United States courts have yet to protect it as a form of political speech, some activists consider DDoS a valid form of 'electronic civil disobedience' (Critical Art Ensemble, 1996). In 2012, Anonymous successfully DDoSed a tear-gas manufacturer in Pennsylvania, the Nasdaq stock exchange, the U.S. Department of Justice, the Federal Bureau of Investigation, the Central Intelligence Agency, the White House, Amazon, eBay, CNN, Buy.com, Paypal and Yahoo, and websites of the Tunisian and Syrian governments (Sauter, 2013). Anonymous used a special tool to successfully DDoS enemies. The low-orbit ion cannon (LOIC) is simple software that sends repeated website refresh requests. It 
'opened the tools and mechanisms of protest organizing and action to the population of the Internet' (Sauter, 2013: 2). Like mirroring, with LOIC anyone can participate.

One feature of LOIC, was a button titled 'fucking hive mind mode' that allowed users to let their computer be controlled by a remote computer. This software gives us insights into the structure of the living network. As Coleman (2012a: np) states, Anonymous embodies 'meritocratic populism' while also resisting 'possessive individualism'. Mirroring is not static but a practice of 'rhizomatic nomadic warfare' (as one of my informants, THXi330, said paraphrasing Deleuze and Guattari 1987) with sudden, ephemeral, and nodal emergences. LOIC, and the 'hive mind' functionality in particular, make evident how Anonymous functions as a rhizomatic living network constituted not by nodes but by edges and the "motors of affective energy" (Lazzarato, 2007: 115). Likewise, mirroring is a practice of a living network alloyed by affect, distinguished by movement across a topology. While 'living network' is a useful heuristic with which to understand the social form of Anonymous it does not account for the importance of shared symbolism.

\section{Improper Names}

Anonymous is not, actually, anonymous. It is pseudonymous. Like the Luddites taking on the name of fictional character Ned Ludd, Anonymous is an 'improper name' (Deseriis, 2013: 35), a shared pseudonym with common symbolic traits. 'Anonymous', is a symbolic system, that enables participants to recognise each other and permits a process of 'subjectivation' (Deseriis, 2013: 35). The concept of subjectivation, or the construction of subjectivity, is key to understanding the unitary power of the improper name and requires an understanding of the term 'dividual' (Deleuze, 1992). Social media firms decompose individuals into separate 'dividual' transactions, metrics, preference, and discreet acts (Deseriis, 2013: 35). An inclusive living network with an improper name such as Anonymous, 'resists capital's attempts to break down the continuity of social praxis in dividual and discrete units' (Deseriis, 2013: 46). The resistance transforms dividuals into collective codividuals. The shared visual and aural symbolism surrounding the improper name Anonymous-most notably the Guy Fawkes mask and video aesthetics-constitutes the codividual symbolism of this living network. Mirroring is a practice that propagates this symbol.

Anonymous has an extraordinarily rich symbolic culture. For an 'improper name' with no official spokesperson, their graphic expressivity provides some of the densest displays of their values. As Coleman (2010: $\mathrm{np}$ ) asserts, '[i]ts aesthetic also helps ensure some 
coherence....while anyone can claim to be Anonymous, you will likely [be] more credible if you follow and play with established patterns.' The smiling black-on-white Guy Fawkes mask popularised in the 2005 movie $V$ for Vendetta is their most prominent symbol representing anonymity, playfulness, and resistance. Distinguishing characteristics of the shared symbolism of Anonymous videos include masked figures, the introductory logo, the text-to-speech (TTS) narration, intense classical or techno music, and the traditional concluding remarks 'we don't forgive, we don't forget, expect us.' Some videos have the quality of masked newscasters, others of hostage videos. The writing is always pedantic and serious, using either first person (us) or second person (you) modes of address. Other than the necessary YouTube account pseudonym, never are authors cited.

Most videos are quite simple, featuring the opening circular and rotating Anonymous logo, a masked figure gesturing, and a TTS intoned declaration. Some are very ornate requiring highly skilled editing. Three of these elements, the logo, icon, and the TTS tonality, are transposable and transferable assets capable of being appropriated to speak any declaration. Anyone can download and edit together the globe spinning preroll, a Philip Glass soundtrack, a gesticulating character in a Guy Fawkes mask, a TTS voice program, an apocalyptic text-and produce an Anonymous video. These foreboding visuals, robotic sounds, and ominous narratives form much of the shared symbolism of Anonymous as an 'improper name.'

World United Anonymous describes Anonymous as an improper name when he directly addressed me, saying:

By default you are already Anonymous, Anonymous in general is an idea, as said before, everyone is entitled to an idea. If you choose to use the name, you can help by spreading knowledge, making videos, music, tweeting, just being a good person, etc.... The name 'Anonymous' just helps put all our ideas in one place so people find them easily. Anonymous is not a group, nor is it an army, person, or even a movement. Anonymous is simply a main idea which is home to a collective of ideas.

'Anonymous' is a pseudonym anyone can adopt that provides the conditions for 'generativity, democracy, and instability' (Coleman, 2010: np). Mirroring is an act of visual resistance that distributes this symbolic system against the threat of deletion.

As a form of cloud activism, Anonymous is entangled with the platform-owning corporations 
they resist. As a living network, they have a fluctuating topology formed by the circulation of affect. The improper name 'Anonymous' is constituted by shared symbols. Like DDoSing, mirroring is a cultural practice specific to this formulation of radical media paradoxically dependent as it is on cloud infrastructure, the circulation of affect, the hope of political mimesis, and a shared pseudonymous symbolism. Mirrors are not mere representations. They mark a will to visibility and chart a terrain of diffraction.

\section{Conclusion}

Caught once again looking at mirrors, it is either the face of transcendence or our own image. It is as if there are no alternative ways to conceptualize matter: the only options seem to be the naiveté of empiricism or the same old narcissistic bedtime stories (Barad, 2003: 827).

Cloud firms may assume the 'naïvete of empiricism' (Barad, 2003: 827) or 'naïve realism' (Gaines, 1999: 93) where database mirroring is confidently practiced and asserted to gain investor and client trust that files will not be deleted or slow in opening. In this case, mirroring is logically positive, an instrumental practice of duplication, and a form of mimicry not mimesis. Barad calls for ways of discussing the world without simplistic reductions to mimicry. We need neither to empirically conceptualise mirrors as accurate copies nor must we consider them as constructed reflections. This article has considered a third way of interrogating mirroring within cloud activism by a living network with an improper name. Mirroring is a praxis of a living network, neither realistic nor representational, but a way of sharing affect, propagating collective symbols on cloud platforms, and hailing codividuals towards political mimesis. Anonymous uses the affordances provided by social media firms to challenge scopic regimes (Jay, 1992) that would silence it. This will to visibility is shared by a number of high-profile cloud activists. Along with the Pirate Bay, the Pirate Party, and Wikileaks, Anonymous may form a 'freedom of information movement' (Beyer, 2014). Mirroring, as an adaptive way of resisting erasure while exploiting the properties of proprietary social media, may become a key practice of this new social movement.

\section{Author Biography}

Adam Fish is a social anthropologist of digital culture, business, and politics at Lancaster University. He investigates the interface of economic and political power, cultural 
discourses and practices, and networked communication technologies. These interests coalesce into critical and ethnographic investigations into media industries and media activism.

\section{References}

AnonymousFacts. YouTube (nd) https://www.youtube.com/user/AnonymousFacts

Anonymousworldwar3. 'ANONYMOUS - A Call For Video Mirroring', YouTube (2010), https:// www.youtube.com/watch?v=MBkGvPTDryw

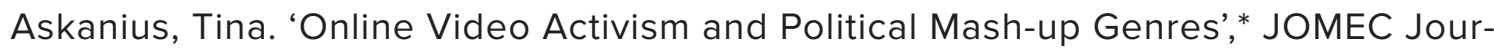
nal Journalism Media and Cultural Studies *(2013) https://lup.lub.lu.se/search/publication/4180637

Barad, Karen. 'Posthumanist Performativity: Toward an Understanding of How Matter Comes to Matter', Signs: Journal of Women in Culture and Society 28.3 (2003): 801-831.

Beyer, Jessica. Expect Us: Online Communities and Political Mobilization (Oxford: Oxford University Press, 2014).

Blue, Violet. 'Anonymous Hacks US Sentencing Commission, Distributes Files', ZDnet (2013) http://www.zdnet.com/article/anonymous-hacks-us-sentencing-commission-distributes-files/

Brighenti, Andrea. 'Visibility: A Category for the Social Sciences', Current Sociology 55.3 (2007): 323-342.

Caldwell, John. Production Culture: Industrial Reflexivity and Critical Practice in Film and Television (Durham: Duke University Press, 2008).

Christiansen, Christian. 'Political Documentary, Online Organization, and Activist Synergies', Studies in Documentary Film 3.2 (2009): 77-94.

ChurchOfScientology. 'Message to Scientology', YouTube (2008) https://www.youtube.com/ watch?v=JCbKv9yiLiQ

Coleman, Gabriella. 'The Aesthetic Face(s) of Anonymous', Savage Minds (2010) http://savageminds.org/2010/12/15/aesthetic-face-of-anonymou/

Coleman, Gabriella. 'Our Weirdness Is Free, The logic of Anonymous-Online Army, Agent of Chaos, and Seeker of Justice', Triple Canopy (January 2012a), http://canopycanopycanopy.com/issues/15/contents/our_weirdness_is_free

Coleman, Gabriella. Coding Freedom: The Ethics and Aesthetics of Hacking (Princeton, N.J.: Princeton University Press, 2012b).

Coleman, Gabriella. Hacker, Hoaxer, Whistleblower, Spy: The Many Faces of Anonymous 
(London: Verso, 2014).

Couldry, Nick. Why Voice Matters (London: Sage, 2010).

Crawford, Kate and Tarleton Gillespie. 'What is a Flag For? Social Media Reporting Tools and the Vocabulary of Complaint', New Media and Society (2014).

Criado, Felipe. 'The Visibility of the Archaeological Record and the Interpretation of Social Reality', in Ian Hodder, Michael Shanks, Alexandra Alexandri, Victor Buchli, John Corman, Jonathan Last, and Gavin Lucas (eds). Interpreting Archaeology: Finding Meaning in the Past (New York: Routledge, 1995).

Critical Art Ensemble. Electronic Civil Disobedience and Other Unpopular Ideas (New York: Autonomedia, 1996).

Dahlgren, Peter. 'The Internet, Public Spheres, and Political Communication: Dispersions and Deliberation', Political Communication 22.2 (2005): 147-162.

Deleuze, Gilles. 'Postscript on the Societies of Control', October 59 (1992): 3-7.

Deleuze, Gilles and Felix Guattari. A Thousand Plateaus: Capitalism and Schizophrenia (Minneapolis: University of Minnesota Press, 1987).

Denton, Nick. 'Church of Scientology Claims Copyright Infringement', Gawker (2008) http:// gawker.com/5002319/church-of-scientology-claims-copyright-infringement

Deseriis, Marco. 'Improper Names: Collective Pseudonyms and Multiple-Use Names as Minor Processes of Subjectivation', Subjectivity 5.1 (July 2012): 140-160.

Downing, John. Radical Media (London: Sage, 2001).

Ernesto. 'Pirate Bay Founder Gets Ready to Run for European Parliament', Torrentfreak (2014), https://torrentfreak.com/pirate-bay-founder-peter-sunde-arrested-sweden-140531/

Featherstone, Mike. 'Body, Image and Affect in Consumer Culture', Body \& Society 16.1 (2010): 193-221.

Fraser, Nancy. 'Rethinking the Public Sphere: A Contribution to the Critique of Actually Existing Democracy', Social Text 25.26 (1990): 56-80.

Gaines, Jane M. 'Political Mimesis', in Jane M. Gaines and Michael Renov (eds). Collecting Visible Evidence (Minneapolis: University of Minnesota Press, 1994). Gelernter, David. Mirror Worlds, or: The Day Software Puts the Universe in a Shoebox... How It Will Happen and What It Will Mean (Oxford: Oxford University Press, 1992).

Gillespie, Tarleton. 'The Politics of 'Platforms', New Media and Society 12.3 (2010): 347364.

Gitelman, Lisa, 2011. 'Danial Ellsberg and the Lost Idea of the Photocopy' in Andrew Ekstrom, Solveig Julich, Frans Lundgren, and Per Wisselgram (eds). History of Participatory Media, Politics and Publics 1750-2000 (New York: Routledge, 2011), 112-124. 
Gregg, Melissa and Gregory J. Seigworth. 'An Inventory of Shimmers', in Melissa Gregg and Gregory J. Seigworth (eds). The Affect Theory Reader (Durham, NC: Duke University Press 2010), 1-25.

Gregory, Sam, Gillian Caldwell, Ronit Avni, Thomas Harding. Video for Change: A Guide for Advocacy and Activism (London: Pluto, 2005).

Habermas, Jurgen. The Theory of Communicative Action (Boston: Beacon Press, 1987).

Halliday, Josh and Charles Arthur. 'Wikileaks: Who Are the Hackers Behind Operation Payback?', the Guardian newspaper (2010) http://www.theguardian.com/media/2010/dec/08/ anonymous-4chan-wikileaks-mastercard-paypal

Hansen, Mark B.N. New Philosophy for New Media (Cambridge, Mass.: MIT Press, 2004).

Haraway, Donna. 'The Promises of Monsters: A Regenerative Politics of Inappropriate/d Others in Cultural Studies', in Lawrence Grossberg, Cary Nelson, Paula A Trechiler (eds). Cultural Studies (New York: Routledge, 1992).

Haraway, Donna. Modest_Witness@Second_Millennium.FemaleMan@Meets_OncoMouse ${ }^{\mathrm{T}}$ : Feminism and Technoscience (New York: Routledge, 1997).

Jay, Martin. 'Scopic Regimes of Modernity' in Hal Foster (ed.). Vision and Visuality (Seattle, WA: Bay Press, 1992), 3-23.

Juris, Jeffrey. 'Practicing Militant Ethnography with the Movement for Global Resistance (MRG) in Barcelona', in Stevphen Shukaitis and David Graeber (eds). Constituent Imagination: Militant Investigation, Collective Theorization (Oakland: AK Press), 164-176.

Kelty, Christopher. Two-Bits: The Cultural Significance of Free Software (Durham: Duke University Press, 2008).

Kerr, Dana. 'Wikileaks Endures a Lengthy DDoS Attack', CNet (2012) http://www.cnet.com/ uk/news/wikileaks-endures-a-lengthy-ddos-attack/

Lazzarato, Maurizio. 'Machines to Crystallize Time: Bergson', Theory, Culture \& Society 24.6 (2007): 93-122.

Lessig, Lawrence. Free Culture (New York: Penguin, 2004).

Lindgren, Simon and Jessica Linde. 'The Subpolitics of Online Piracy: A Swedish Case Study', Convergence: The International Journal of Research into New Media Technologies 18.26 (2012): 143-164.

Manovich, Lev.* The Language of New Media* (Cambridge, Mass: MIT Press, 2001).

McLuhan, Marshall. Understanding Media (New York: McGraw-Hill, 1964).

McKelvey, Fenwick. 'We Like Copies, Just Don't Let the Other Fool You: The Paradox of the Pirate Bay', Television and New Media (2014). 
Microsoft. 'Benefits of Database Mirroring' (2014), http://technet.microsoft.com/en-us/library/ms189852.aspx\#Benefits

Milan Stefania. 'WikiLeaks, Anonymous, and the Exercise of Individuality: Protesting in the Cloud', In Benedetta Brevini, Arne Hintz, and Patrick McCurdy (eds). Beyond Wikileaks. Implications for the Future of Communications, Journalism and Society (London: Palgrave Macmillan, 2013), 191-208.

Mouffe, Chantel. On the Political (New York, NY: Routledge, 2005).

Navas, Eduardo.'Regressive and reflexive mashups in Sampling Culture', Remix Theory (2010) http://remixtheory.net/?p=444

Nielsen, Rasmus K. 'The Labors of Internet-assisted Activism: Overcommunication, Miscommunication, and Communicative Overload', Journal of Information Technology \& Politics 6.3-4 (2009): 267-280.

Ong, Walter J. Interfaces of the Word: Studies in the Evolution of Consciousness and Culture (Ithaca, N.Y.: Cornell University Press, 1977).

Parikka, Jussi. 'Copy', in Matthew Fuller (ed.). Software Studies: A Lexicon (Cambridge, Mass.: MIT Press, 2008), 70-78.

Postigo, Hector. The Digital Rights Movement: The Role of Technology in Subverting Digital Copyright (Cambridge, Mass.: MIT Press, 2012).

Sauter, Molly “LOIC Will Tear Us Apart:" The Impact of Tool Design and Media Portrayals in the Success of Activist DDOS Attacks', American Behaviorial Scientist 57.7 (2013): 9831007.

Scott, James. Seeing Like a State: How Certain Schemes to Improve the Human Condition Have Failed (New Haven: Yale University Press, 1999).

Sifry, Micah. Wikileaks in the Age of Transparency (New York: OR Press, 2011).

Stoehrel, Rodrimigo Ferrada and Simon Lindgren. 'For the Lulz: Anonymous, Aesthetics, and Affect', trip/eC 12.1 (2014) http://www.triple-c.at/index.php/tripleC/article/view/503

Taussig, Michael. Mimesis and Alterity (New York: Routledge, 1993).

Thacker, Eugene. 'Networks, Swarms, Multitudes. Part One', Ctheory (2004), http://www. ctheory.net/articles.aspx?id=422.

Tsing, Anna Lowenhaupt. Friction: An Ethnography of Global Connection (Princeton N.J.: Princeton Press, 2005).

Wiedemann, Carolin. 'Between Swarm, network, and multitude: Anonymous and the infrastructure of the common', Distinktion: Scandinavian Journal of Social Theory, (2014) http:// www.tandfonline.com/doi/full/10.1080/1600910X.2014.895768 
Wikileaks.info. 'Mirrors' (nd), http://wikileaks.info/

Wikileaks.org. (nd), https://wikileaks.org/Mirrors.html

Williams, Linda. 'Film Bodies: Gender, Genre, and Excess', Film Quarterly 44.4 (Summer 1991): 2-13.

The LOCKSS System has the permission to collect, preserve and serve this open access

Archival Unit

This Isuue of the Fibreculture Journal by The Fibreculture Journal Incorporated is licensed under a Creative Commons Attribution 4.0 International License.

\section{OPEN HUMANITIES PRESS}

The Fibreculture Journal is published by The Fibreculture Journal Incorporated in partnership with Open Humanities Press. 


\title{
The Fibreculture Journal
}

DIGITAL MEDIA + NETWORKS + TRANSDISCIPLINARY CRITIQUE

issue 26: Entanglements - Activish and dechrology

FCJ-192 Sand in the Information Society Machine: How Digital Technologies Change and Challenge the Paradigms of Civil Disobedience

Theresa Züger

Alexander von Humboldt Institute for Internet and Society

Stefania Milan

University of Amsterdam

Leonie Maria Tanczer

Queen's University Belfast

\begin{abstract}
:
Digital technologies have fostered the rise of new forms of civil disobedience that change and challenge established notions of this form of political action. This paper examines digital civil disobedience using the concept of friction to explore contested entanglements of this kind of protest and its new technological adaptations, as well as tensions on the conceptual level of civil disobedience. The paper is split into in three sections which offer analyses of (a) the historical dimension of this form of protest, (b) seven factors that represent some of the features of contemporary digital forms of civil disobedience, and (c) the recurring motif of power of information within digital civil disobedience. The paper is centered on the notion that transformations of civil disobedience demand a reconsideration of traditional understandings of civil disobedience to meet the conditions of our current society.
\end{abstract}

doi: $10.15307 /$ fcj.26.192.2015 


\section{Introduction}

Oscar Wilde (1909) once wrote that '[d]isobedience, in the eyes of anyone who has read history, is man's original virtue. It is through disobedience and rebellion that progress has been made.' In that sense, civil disobedience, which is a dissident form of political protest (Hahn, 2008: 1365), is embedded in a historical context and enables societal advancement while also leading to public friction. As society faces inequality, global mass surveillance and unequal power dynamics, civil disobedience has certainly not lost its importance in the twenty-first century.

However, due to the development of the Internet and its broad use and deployment, the tactics and tools of civil disobedience have changed. We are witnessing acts of disobedience in both an offline and online context, which highlight the diversity of mechanisms available to citizens to counteract injustices and dissatisfaction. Thus, just as the African-American Rosa Parks helped spark the civil rights movement in the 1950s by disobeying the racial segregation laws concerning buses, certain online acts, for example, those by the hacktivist collective Anonymous, are signs of political resistance.

As the attention paid to such digital actions increases, the question arises of how older, traditional forms of civil disobedience are transformed through the use of the Internet and what the effects of this transition are for both the act of civil disobedience, and also for society in general. The following paper will therefore try to identify the changes and challenges of this transformation and will make use of the concept of friction (Tsing, 2005). This should help to identify the difficulties presented by the new entanglement of activism and technology in the context of digital civil disobedience.

\section{Theoretical Underpinnings and Background}

Civil disobedience is a form of political contestation (Celikates, forthcoming) that aims to address injustice in a broader sense. It opposes not only injustice in distribution but all kinds of democratic deficits (Celikates, 2010: 291). Hence, in some cases, civil disobedience is a vehicle for a deeper critique that finds fault within the design or implementation of democratic processes rather than addressing only one single issue. It opposes tendencies that de-politicise public issues by alienating them from the collective self-determination of the people (Volk, 2013). Civil disobedience seeks to combat the 
democratic shortfalls that result from, for example, abuses of power or illegitimate power, structurally flawed processes, or even more subversive democratic deficits, such as exclusion or a lack of transparency (Kumar, 2013; Celikates, 2010: 291). It has the potential to initiate political transformation where institutions and laws are unable to perform this change due to inertia and their positivistic claim of absolute authority (Arendt, 1972: 101).

Civil disobedience has been theorised among others by Habermas (1985), Dworkin (1985) and Rawls (1972). All three adopted a so-called liberal approach to civil disobedience, though aspects of their perspectives have proven to be highly controversial: as shown by Zinn (1991) and Celikates (2014; forthcoming), who have for instance criticised the general understanding of civil disobedience as a symbolic act, rather than a confrontational act. In contrast to the earlier scholars, Celikates, for example, stands in a tradition of thought called radical democratic theory. Arendt (2012) can be seen as a pioneer of this line of thinking. Following this latter tradition of thinking in our argument, civil disobedience is here defined as:

an intentionally unlawful and principled collective act of protest (in contrast to both legal protest and "ordinary" criminal offenses or "unmotivated" rioting) that (in contrast to conscientious objection, which is protected in some states as a fundamental right) has the political aim of changing (a set of) laws, policies, or institutions (Celikates, forthcoming).

This minimal understanding of civil disobedience does not imply legitimacy per se. Instead, we have to recognise that legitimacy is not inherent to the act, but can only be evaluated ex post and will probably stay politically contested (Celikates, 2010: 294).

Drawing on Arendt's political theory, civil disobedience is not easily determined by a set of criteria that, according to liberal theories, need to be evident when referring to the concept. Instead, civil disobedience gains its quality and political justification for each specific case from the democratic compatibility of these actions, meaning for instance the consideration of plurality and equal rights to political self-determination as a condition of the common world. Motivations such as personal interests or beliefs thereby don't qualify to justify civil disobedience as a political action. On these terms, civil disobedience becomes legitimate by actualising the freedom to politics while at the same time acknowledging a self-limitation of freedom (Kalyvas, 2008: 243). This is rooted in the concern about a shared world between humans and implies respecting your own and other's freedom. 
Having now established a general understanding of civil disobedience, we may now turn to recent developments, which have seen this traditional concept challenged by the usage of digital strategies. Since the 1990s, when the World Wide Web entered our households, the opportunities for engaging in civil disobedience have multiplied, as the Internet has offered a novel terrain for expressing political dissent (Klang, 2008). This has been frequently touched upon in literature on online activism and hacktivism (Taylor, 2004; Hands, 2011; Ziccardi, 2012; Boler, 2008) as well as in single case examples of digital civil disobedience (George, 2013). The concept of digital civil disobedience, thus, expands the original notion, which referred to purely offline action, by transferring it into an online setting through the utilisation of information and communication tools (ICT).

However, this does not imply that civil disobediences simply transpose the tactics used offline to the Internet. Rather, digital tactics of civil disobedience foster the transformation of civil disobedience by changing and challenging the concept with new practices (Wray, 1999). They exploit the infrastructure's technical and ontological features for political or social change (Milan, 2015). We can therefore identify a matrix of tactics used for political actions, ranging from traditional forms of civil disobedience that have gone digital, to those that have emerged from Internet practices.

The Internet's early years were accompanied by the utopian and universal dream of a globally networked public sphere with the capacity to foster equal participation (Gimmler, 2001). Technology and activism were entangled, and online activism was connoted with high aspirations of democratisation and empowerment for civil society (Barlow, 1996). Viewed in light of this earlier literature, the current literature often gives a more ambivalent picture, which omits these universal yearnings (Morozov, 2011; Sifry, 2014). In The Exploit: A Theory of Networks, Galloway and Thacker (2007) portrayed the Internet as a platform for both corporate and subversive activity, due to a structure that is both highly centralised and dispersed. Surveillance, securitisation and commercialisation are increasingly turning the Internet into both an object of contestation in itself, and at the same time a tool and platform for a broad range of other political means.

This highlights the value of casting digital civil disobedience as a story of various forms of frictions (Tsing, 2005). The concept of friction can be understood as a metaphor for the diverse and sometimes conflicting entanglements of our contemporary society, leading to new arrangements of culture and power. This utopian vision of the Internet can reflect 'aspirations of global connection' which come to life in the 'sticky materiality of practical encounters' (Tsing, 2005: 1). However, friction should not be understood as being a synonym for resistance. Instead '[h] $\mathrm{h}$ gemony is made as well as unmade with friction' (Tsing, 2005: 6). 
The broad concept of civil disobedience in itself can be comprehended as a friction phenomenon, as state legislation or political measures collide with a dissenting claim for self-determination by citizens. Digital disobedience adds new critical momentum to this already tense situation and gives visibility to friction on two new levels. Firstly, it adds critical moments on the level of entanglements of activism and technology, as offline activism does not simply convert to digital equivalences. Secondly, there is also a less visible kind of friction that challenges the conceptual level of civil disobedience. These two aspects will be discussed in this paper more closely.

Overall, on the basis of the outlined arguments, the present paper aims to investigate a new diversity of approaches, objectives and articulations of civil disobedience, which are new points of encounters causing friction. Many of the current acts of digital civil disobedience are concerned with the (re-) conquest of power over information against the state or a private authority. This is done by either encrypting, manipulating, inventing, or distributing information. The recent transformation of civil disobedience requires taking a closer look at the nature of the digital tactics to facilitate an informed discussion about their justification. Both of the aforementioned two dimensions of friction-the action/ technology level as well as the conceptual level-will therefore be explored and interlinked throughout this paper using selected examples of new forms of civil disobedience.

The paper itself is split into three parts. The first section briefly examines the historical dimension of digital civil disobedience starting with the earliest encounters of this form of protest. The second section highlights the new conditions of digital civil disobedience as observations that serve as a challenge to traditional forms of civil disobedience. It comprises seven specific factors including (a) semiotics, (b) automatisation, (c) individuality versus collectivity, (d) new formations of action, (e) anonymity, (f) publicity, and (g) asymmetry. We thereby acknowledge that the previously mentioned key principles of civil disobedience remain in existence. The third and last part of the paper identifies power of information as a recurring motif of digital civil disobedience. This is discussed in reference to the example of political whistleblowing. These aspects help to explore digital civil disobedience from a novel perspective.

\section{A Brief History of the Emergence of Digital Civil Disobedience}

Digital civil disobedience emerged long before the advent of the World Wide Web. In 1985, the Berlin-based hacker collective known as the Chaos Computer Club (CCC) exploited a flaw in the German Bildschirmtext home terminal system to raise awareness of its security 
risks (Denker, 2013). The Bildschirmtext was an interactive videotext system used for making payments and was operated by the West German postal service. CCC members hacked into the system, organising a massive transfer of money in their favour. However, in contempt of the expectations that this act was motivated by a driving self-interest, the CCC did in fact return the money one day later during an ad hoc press conference. This event clearly highlights how this symbolic but confrontational act had the intention of transmitting a political statement, despite the fact that the action itself was against the law. Hence, the collective effort of the CCC was aligned with the common interest of ensuring data protection and security of the system (Danyel, 2012). The fact that the CCC also made the action public to foster an informed debate emphasises the notion of an act of civil disobedience.

In 1996, the US tactical media collective Critical Art Ensemble (CAE) was the first to conceptualise the idea of electronic civil disobedience (Wray, 1999). This term started out dissociated from existing theories and was inspired but not embedded within existing concepts of civil disobedience. Nevertheless, in a series of influential publications, CAE activists declared electronic civil disobedience the most meaningful form of political resistance in light of the distributed power typical of late capitalism. As the very nature of power changed, they argued, traditional embodied forms of civil disobedience would lose traction, especially as authorities became more efficient in 'evad[ing] the provocations of C[ivil] D[isobedient] participants' (Critical Arts Ensemble, 1996: 9). Electronic civil disobedience was at that time thought of as 'another option for digital resistance (...) that would produce multiple currents and trajectories to slow the velocity of capitalist political economy' (Critical Arts Ensemble, 2001: 13-14).

Rather than attempting to create a mass movement, CAE activists envisioned electronic civil disobedience as a cell-based hit-and-run media intervention. It sought to disempower power-holders through symbolic disturbance and the corruption of information channels. It was assumed that blocking the flow of information within an organisation would disturb all its operations (Critical Arts Ensemble, 1996). Even though the CAE explained the primary tactics as being 'trespass and blockage' and the 'same as traditional civil disobedience' (Critical Arts Ensemble, 1996: 18), their model represents-to a certain extent-an inversion of the classical model of civil disobedience. It substituted the notion of the rebellious mass with a 'decentralised flow of particularised micro-organisations' (Tactical Media, n.d). At that time the CAE was aware that electronic civil disobedience could be misused. They therefore set up a kind of behavioural codex. The conditions they emphasised were based on guaranteeing no harm to humanitarian infrastructures or data, and abstaining from targeting individuals. 
Although the CAE never tested their concept, relatively soon other activist groups experimented with disobedient tactics that drew on this understanding of political protest. The first experimental setting used by different activist groups was the distributed denial of service (DDoS) attack (Sauter, 2013: 1). One of the first incidents of a DDoS action dates back to September 1995, when the then-French President Jacques Chirac announced that France would run a series of nuclear tests on the Polynesian atoll of Mururoa. As a consequence, a group of Italian activists organised an attack against the websites of the French government to voice their opposition. The call for action invited people to join:

a demonstration of 1,000,10,000,100,000 netusers all together making part of a line crossing French Government's sites. The result of this strike will be to stop for an hour the network activities of the French Government (Tozzi, 1995).

On December 21, 1995 ten websites were targeted simultaneously by thousands of users who continuously reloaded the page. The process made the websites temporarily unavailable. This so-called netstrike was according to its promoters meant to be 'the networked version of a peaceful sit-in' (Milan, 2013a: 47).

The following year, a group of Italian activists-allegedly the same group as beforepublished a 145-page book designed to spread the tactic. The first chapter, titled NetStrike Starter, included a detailed explanation of how to organise this specific form of protest (Strano Network and Tozzi, 1996). Consequently, many netstrikes followed across the world. They typically targeted governmental websites and opposed, for example, the death penalty and the war in the former Yugoslavia (Bazzichelli, 2008: 171). The underpinning idea of the netstrike is still in use in present day actions.

In 1998, the Electronic Disturbance Theatre (EDT) was created as a spin-off of CAE and in support of the Zapatista struggle in Mexico (Paris and Ault, 2004). Amongst their various actions, EDT activists launched a piece of software called FloodNet (Tanczer, 2015). This was an $\mathrm{HTML} /$ Java applet that sent automatic requests to reload a target page, overexerting the server/website. In that sense, the tool enabled a more automated way of running a DDoS attack. These specific incidents further highlight an interconnectedness of offline and online forms of digital disobedience, as EDT's call for action was designed to encourage both tactics. In addition, the tool strategically translated a known tactic of civil disobedience from the offline to the online world, through the understanding of DDoS attacks being an equivalent to physical sit-ins. 
Additionally, much of the street disobedience of the 1990s and early 2000s was made possible by alternative Internet Service Providers (ISPs). They functioned as the digital backbone of the emerging transnational social movements. They did not hesitate to disobey existing legislation to protect fellow activists (Milan, 2013a), although at present, this seems to be no longer the case. For example, in 2006 the European directive (2006/24/EU) forced providers of electronic communications to retain users' metadata and release them to the authorities upon request. This is in open contradiction with the principles of alternative ISPs. As a consequence the ISPs decided not to comply, and instead tried to create technical bypasses and awareness amongst their users. The communiqué released by participants in an anti-data retention workshop in Budapest called for civil disobedience by providers and users alike. The directive was accused of exercising 'pre-emptive surveillance of communication structures' and of forcing providers 'to work as outsourced police forces' (Milan, 2013a: 156). It concluded with the following provocation: 'We will pour as much sand into this machine of suspicion as we possibly can' (Milan, 2013a: 156). This shows how the struggle about the authority over information was not only fought using individual practices but also at the institutional level.

The examples provided indicate how digital civil disobedience-over fifty years after Rosa Parks and almost twenty years after CAE's vision-has become a common practice for activists across the globe. More recently, debates around the decentralised online community Anonymous have revived the concept. They resuscitated the idea of digital sit-ins and launched an online disruption campaign of DDoS attacks, 'protesting peacefully for freedom of expression on the Internet' (Colby, 2010). Political aspirations within Anonymous slowly crystallised in what started off as a loose assembly on the 4chan online forum (Sauter, 2014). Although initially primarily concerned with pranks or jokes (Coleman, 2013), the Anonymous collective increasingly mobilises against governments, companies and individuals in retaliation for behaviours they believe are harming society or cyberspace (Coleman, 2014; Sauter, 2014). Some observers have saluted them controversially as 'the new guardian of our civil liberties' (Coleman, 2013), others have equated them with 'armchair cyberwarriors' (Warner, 2010) or simply criminals, to the point that the 2011 NATO Spring Report listed them among cybersecurity threats (NATO, 2011).

\section{The Novel Frictions of Digital Civil Disobedience}

The above stated contestation around Anonymous highlights the ongoing controversies around the concept of (digital) civil disobedience. As this form of protest has expanded its scope and tactics, new disputes on the justification of civil disobedience have been 
created. In the following section we will trace and compile some of the factors influencing the changes and challenges of civil disobedience when shifting this form of protest to the online sphere. Starting off from the historical context described above, it is an explorative process to examine arising frictions, such as rejection or even sanctions against civil disobedience. This process will also highlight unintended consequences and open questions, of which we would like to name a few.

\section{Semiotics}

On a semiotic level, the Internet entails new conditions of communication and visibility for civil disobedience. Traditional practices of civil disobedience combine a communicative level and confrontational level of action (see Raz, 1994: 264). Although in instances of digital disobedience, the physicality of presence and action is not simply replaced by virtuality, the modes of representation and action have shifted. Whereas, in the past, speech took the form of written or spoken words and body language, it becomes code and pixels in the digital realm. In other words, we examine a transformation of the symbolic format of civil disobedience. New performative strategies of civil disobedience are increasingly adopting technologically mediated appearance. Traditional physical presence and tangible disruption are now transformed into resistance exploiting the architectural and the information level of the Internet.

One of the chosen sites for this struggle is the disobedient use of semiotics, meaning the disobedient use of signs and symbols, content or code. These often represent a certain purport of an action. It therefore implies the manipulation of content such as graphics, text or pictures as in the case of political website defacements (Klang, 2008: 77) or so-called 'e-graffiti' (Auty, 2004: 216). The tactic can be exemplified through the Spying Birds incident, whereby the* website of the software company behind the game Angry Birds *was hacked. This happened as a reaction to claims that intelligence agencies had collected data through the exploitation of the smartphone app (Gibbs, 2014). The manipulation plays on the initial meaning of the video game, while using the alteration of the name and website to express dissent. In addition, it demonstrates a contest not only over freedom of speech and different versions of perceived truths, but also over access to network infrastructure and IT-security.

A change introduced by this semiotic shift concerns the visibility and appearance of the actor. While in many traditional forms of disobedience a person with her physical materiality becomes and constitutes part of the dissident action, this is no longer the 
case in the online setting. For example, a protester chaining herself to a train track is more substantially linked to the dissident product or action than in digital forms of civil disobedience. Thus, while the defacement of* Spying Birds* is visible, it is detached from the person(s) performing the dissident action. Communicative effort and the appearance of the author are not intertwined online.

\section{Automatisation}

A further factor and friction is that novel technological developments confront political action through automatisation. Specifically, the utilisation of software such as the DDoS tools FloodNet or the more recent Low Orbit Ion Cannon (LOIC; Sauter, 2014) highlight this trend. It defies traditional notions of civil disobedience by reconsidering the role of technical actors in the course of political encounters. This poses concerns on the deliberateness or reflection of protesters.

Civil disobedience is characterised by intentionality and enacted principles. An argument made against the use of DDoS tools is that the availability and usability of such instruments leads to unreflective impulsive decisions. Intentions, consciousness or strategic goals are difficult to evaluate from an outsider or retrospective point of view. 'Risk and cost are relative to the experience of the individual' (Halupka, 2014: 118). Therefore, intentionality cannot be determined objectively.

Furthermore, it would be short-sighted to understand DDoS actions purely as a form of clicktivism, meaning 'low-risk, low-cost activity via social media whose purpose is to raise awareness, produce change, or grant satisfaction to the person engaged in the activity' (Rotman et al., 2011: 3). DDoS actions neither come at a low cost nor at a low risk. This is clearly evident from a legislative standpoint, according to which these acts are considered felonies.

Aside from this, Foredyke (2013) introduces the question of the disappearance of human subjects in automated political action:

[w] hen the idea of networked political dissidence is compounded with the automated functions of software and hardware devices that are necessary to 
mobilise in networked political dissent, then explicit human subjects start to disappear (Foredyke, 2013: 6).

Foredyke raises the issue of over-prescribing intent to DDoS attackers and points to the lack of a connection or disconnect between the automated act and statements about motives.

Moreover, the automatisation of digital civil disobedience leads to an increased use of certain tactics. This is specifically evident in the case of DDoS (Nazario, 2008). In spite of civil disobedience often being conducted as a creative and unique action to transmit a political message, the inflationary use of a method can affect the political value of a tactic. The quality of its performative character is crucial for its political impact. Frequent and common utilisation of DDoS may disregard the substance of each individual political claim. Increasing automatisation of civil disobedience might therefore call for a re-evaluation of the trade-off between a unique political message and the cost of ordinariness.

\section{Individuality Versus Collectivity}

According to Arendt (1972: 74), collectivity is a crucial part of what defines civil disobedience as a truly political act. She argues that civil disobedience can never be an isolated action in the interest of a single individual. This notion of the individual agent who is following a higher law or her personal conscience, which is being perceived as superior to legislation, is an ambiguous idea within the longstanding tradition of the philosophical discourse on civil disobedience. It can be traced back to Thoreau (1993) who is mistakenly considered the originator of the term civil disobedience (Laudani, 2013: 94).

Arendt (1972: 60) rejects Thoreau's actions as examples of civil disobedience, since for her, the individual conscience is a highly subjective and isolated entity that does not owe anyone but the self. Celikates (2010: 282) builds on this notion and calls this argument of legitimising civil disobedience the individualistic-romantic model and criticises it as fundamentally apolitical. Online actions performed individually, such as coding or launching a software program, can occur in complete isolation independently of a collective. They therefore pose the question if acts that are conducted by individuals can be categorically disqualified as civil disobedience. 
Kalla (1986: 266) points out that it is not the quantity of actors that is decisive for the quality of the political act. Instead, the notion of civil disobedience is determined by the notion that an individual acts on behalf of a public and collective interest. Therefore, even individual acts of political hacking can be seen as civil disobedience in the case that they enact a common interest. Nevertheless, there are incidents where an individual is acting in isolation and in pure self-interest when conducting a dissident act. This would certainly not be compatible with the spirit of civil disobedience. Moreover, the debate around individuality and collectivity is further complicated by the fact that intentions of individuals are often highly subjective and not necessarily uncontroversial. Hence, an individual might potentially act on personal interests but believes herself to be acting on behalf of common concern.

\section{New Formations of Action}

Political phenomena such as Anonymous promote new formations of online protest. These expand and defy conventional notions of collective action (Postmes and Brunsting, 2002; Calderaro and Kavada, 2013). Within the literature, some forms of contemporary digital disobedience are associated with concepts such as connective action (Bennett and Segerberg, 2012) or cloud protesting (Milan, 2013b). Connective action is regarded as 'personalised collective action formations in which digital media become integral organisational parts' (Bennett and Segerberg, 2012: 760). Likewise, the cloud is a metaphor for a specific way of connecting individuals in joint action that are supported both materially and symbolically by the Internet (Milan 2013b: 200).

The symbolic production mediated by the Internet allows for direct and flexible participation by a variety of individuals. Coleman (2014), who frames Anonymous as a mature and serious political movement, notes that:

Anonymous is emblematic of a particular geography of resistance. Composed of multiple competing groups, short-term power is achievable for brief durations, while long-term dominance by single group or person is virtually impossible (Coleman, 2014: 393).

Her description rejects the idea of an anarchic coalition as stated by Shantz and Tomblin (2014), pointing out '[w]ithin each network there are certain participants who can allow or disallow certain people (...) it kind of keeps people in line on that network. So there are 
forms of control' (Garfield, 2011). Coleman (2014: 395) concludes that 'organising structures can never quite be apprehended, Anonymous is composed of people who decide together and separately to take a stand'. Thus, this newly gained flexibility for political protest through the Internet allows participants to tailor their engagement more specifically.

While these formations of actions may be seen as offering new potential for digital civil disobedience, they can also be perceived as friction within the public political discourse. If the formation consists of a flexible association of individuals, the question arises as to how reliable the multitude of internal political opinions will be in the public's eye. 'Anonymous simultaneously enacts liberation and control, dissent and a lack of accountability, privacy and piracy' (Ravetto-Biagioli, 2013: 190). The risk that this plurality of agendas and loose affiliation brings with it is that it might be questioned regarding its credibility or at least come with higher cost for the actors when they seek to gain public trust and support.

\section{Anonymity}

The anonymity chosen by some disobedient actors in the context of online actions, poses a challenge for the concept of civil disobedience. In traditional forms of civil disobedience, actors either freely expose their identity or one can assume they at least face a constant risk of their identity being exposed. In the context of the Internet, identifiability is not necessarily a prerequisite. In fact, as various examples show, the position towards anonymity is controversial among different online activist cultures.

For instance, EDT activists are very keen to emphasise that they use their real names when engaging in disobedient actions. They base this on the understanding that '[electronic civil disobedience] is about bringing together real bodies and digital bodies in a transparent manner which is the same tradition as civil disobedience' (Dominguez, 2005). This is different within Anonymous, depending on a collective moniker as a 'floating signifier, or rather a signifier of something that is existing but rather undefinable' (Ravetto-Biagioli, 2013: 180). In this way, different activist groups choose to either use or refrain from anonymity in the course of their political activities.

However, digital anonymity, despite its insecurities and limits, offers new abilities and also lends new qualities to these kinds of actions. In sociological terms, anonymity thereby serves the purpose of fostering solidarity through flexible identification (Milan, 2013a). In 
the course of an act of digital civil disobedience, the protagonist puts herself in the rear. As a consequence, the action itself comes to the fore. Coleman states in the course of an interview that anonymity 'is a kind of political gateway for a lot of geeks who may have not participated in politics before' (Garfield, 2011).

The use of anonymity highlights a tension with the earlier models of civil disobedience and public political discourse. Although we acknowledge the relativity of anonymity online, certain anonymising technologies impede the authorities in tracking down digital disobedient acts. From a liberal theoretical perspective this is relevant in regard to legitimacy. This school of thought argues that the acceptance of the punishment is decisive for the legitimacy of the disobedient act (Rawls, 1972: 404). In opposition to this notion, Arendt (1972: 67) finds it:

most unfortunate, that in the eyes of many, a "self-sacrificial element" is the best proof of "intensity of concern" (...) for single-minded fanaticism is usually the hallmark of the crackpot and, in any case, makes impossible a rational discussion of the issue at stake.

Thus, we may acknowledge that there are circumstances under which anonymity might be the only way to protect oneself from unjust and disproportional penalties while still articulating protest within one's action.

Yet, anonymity comes at the cost of hampered accountability (Davenport, 2002). Even if one might not agree with Davenport's (2002: 35) plea to 'embrace accountability and reject anonymous communications' anonymity at least comes with a strategic obstacle for civil disobedience: anonymity obscures political actors to the civilian community and impedes a process of understanding, identification and credibility of actors and actions. This becomes clearly evident in the fact that anyone could potentially claim to act in the name of Anonymous and as stated by Foredyke (2013: 18) and Mansfield Devine (2011), a majority of the most influential attacks seem to have in fact been organised 'outside of the input of the community'.

The risks associated with anonymity are exemplified by the case of Anonymous infighting, where certain groups have claimed to be more authentic than others (Stöcker, 2014). The case that Coleman (2014: 337) calls 'Sabutage', where she refers to a member cooperating with the FBI who went under the handle Sabu, also shows that accountability and trust are not only relevant for the public, but that an anonymous network can also suffer 
internally from traitors or spies. [1] Hence, in many cases of political action, anonymity does not compromise the justification of civil disobedience but it often comes with strategic obstacles and costs that ask for thorough consideration.

\section{Publicity}

Digital acts of civil disobedience have shifted the ways in which dissident tactics are publicised. This is underpinned by the understanding that communication is a crucial element of this form of protest (Brownlee, 2007). This becomes particularly important in instances where the dissident disruption targets the architectural level of the Internet, as is the case with DDoS attacks. In such case, the intention or goal of the civil disobedience often remains invisible to the average user, which is why an explicit articulation of the rationale and reference to the movement helps to create awareness among the public.

For Brownlee, the legitimacy of civil disobedience is influenced by the quality of communication (2012: 7), which encourages actors to articulate the dissident act to the public via various channels such as press releases, Twitter, videos, or websites. The communicative effort can help to explain motives or authorship of an action (Garfield 2011). The concept of publicity has also shifted in regard to the phase in which the disobedient act is revealed to the public. Unlike traditional liberal understandings of civil disobedience (Rawls, 1972: 366), some digital acts 'depend on* not* giving authorities advance notice' (Celikates, 2014: 213). To take this argument a step further, some acts of digital civil disobedience even depend on secrecy**as a breach of law occurs, which is specifically relevant for dissident acts such as whistleblowing or website defacements.

Thus, public involvement of digital civil disobedience must often be created after the actual event of civil disobedience has taken place. Although this is not a new feature of civil disobedience, the fact that online actions often require some sort of technological knowledge highlights the necessity to articulate very clearly the method and intention of the dissident act to fellow members of civil society. This necessitates an informed, objective, and credible intermediary communication channel. Without additional media reports acts of digital civil disobedience potentially stay invisible, and remain or become (mis)understood. 
Another friction between civil disobedience and the public are novel strategies of performance. In order to subvert and/or take advantage of the ongoing spectacularisation of politics by mass media (Mazzoleni and Schulz, 1999), activists have occasionally purposively promoted a cross-fertilisation of civil disobedience with the arts. This is used as yet another step to draw attention to the values they promote or protect. For instance, EDT created the Transborder Immigrant Tool, which enables the use of technology to support refugees and prevent deaths on the US-Mexican border by making GPS-maps of water caches available to immigrants (Tanczer, 2015). This project of the EDT led to an investigation by the FBI Office of Cybercrimes and a firestorm of political controversy in the USA (Nadir, 2012). Despite its inherent practical purpose and intent, the operation has been exhibited in the Museum of Contemporary Art San Diego as well as in the Orange County Museum of Art (Bang Lab and Electronic Disturbance Theatre 2.0, n.d.).

Similarly, servers running* as TOR relays-systems that enable private, unsurveilled networked communications-were hosted at the Reina Sofia Museum in Madrid as part of the Really Useful Knowledge* exhibition in 2015 (Museo Nacional Centro de Arte Reina Sofía, 2014). [2] This interconnectedness of civil disobedience with art practices is embedded in early notions of electronic civil disobedience, and enacted by groups like EDT. It is noteworthy that the EDT used the term theatre to describe their collective, as the term is historically framed as a civil institution educating society (Hentschel, 2000). This connection of EDT with the notion of theatre brings in play means of dramatisation, illusion and catharsis of performance.

Moreover, the perception of having one singular target audience is being questioned. Theorists increasingly reject the idea of the public as a solitary realm of deliberation. Instead, they announce the emergence of plural digital publics (Bunz, 2012; Münker, 2012) understood as 'more dynamic, diverse, decentralised, and effective alternative networks of communication' (Benkler et al., 2013: 10). The concept of the networked fourth estate (Benkler, 2013) also points out that the audience addressed by digital disobedient actors is not necessarily locally restricted but globally distributed. In addition to being occasionally transnational in nature, these publics sometimes gather quite spontaneously in what has been called 'flash publics' (Bratich, 2012; Schwarz, 2014: 185). The public perception of digital civil disobedience is therefore not restricted to* local witnesses* or a media report. Yet, the question arises whether this decentralised public still has to be newly understood in the political context. 


\section{Asymmetry}

The final factor of friction which we will discuss, is the asymmetrical relation between the perceived threat and the activist intentions, evident in some cases of digital civil disobedience. An EDT member stated that governments responded to their actions 'as if it was a real threat to them (..) it is being treated as if it is a serious, real attack like a bomb' (Tanczer, 2015). This is an interesting claim, considering that the first FloodNet actions back in the 1990s were not then defined as illegal. In the course of this interview the EDT member further describes that

[t]he media was sensationalising what we were doing, while in fact all we wanted to do is to bring attention to a particular event or situation. We were not really trying to-you know-bring down companies' (Tanczer, 2015).

This highlights a securitisation of the dissident act that equated the political action with a perceived security threat.

This phenomena has already been observed with hacktivism and the securitisation of Anonymous (Dunn, Cavelty and Jaeger forthcoming). Coleman (2014: 394) assumes 'it may be the potency and the politically motivated character of the groups' actions that prompts the state to so swiftly criminalize them.' As a consequence, the dissident action or actors pose a threat to certain bases of the state's ontological existence and challenges its claim to unrestrained surveillance (Dunn Cavelty and Jaeger, forthcoming). The asymmetry is also evident in the tactic of website defacements.

In this context, Franklin (2001) highlights that from an IT-security perspective there exists a potential to create greater damage than the defacement itself, for example, financial loss or data breaches. This potential exists, even though the actor might not exploit it. From a legal perspective, political defacements are not distinguished from other, more self-interested website defacements with malicious or criminal intent. This legal one-dimensionality again induces an asymmetry between perceived threat and political intent.

Furthermore, one could argue that website defacements are a logical evolution of culture jamming, which refers to the defacement of political or commercial messages on billboards and public wallpaper long before the Internet (Deitz, 2014). The qualitative difference 
between the two evolves out of the need to have server access to enable a content manipulation in the course of a website defacement. This far-reaching access into a system the actor is trying to affect highlights how novel digital actions come with their own logic and frictions, and therefore require case-by-case assessments.

\section{Power of Information}

The dynamics we have just outlined-those that affect the digital tactics of civil disobedience-can all be considered under the overarching idea of the power of information. Notions such as information society, information age (Webster, 1995) or simply* information and communication technologies* (Castells, 2000) highlight the value and power of information in our current societies. Among other practices, civil disobedience has benefited from new and emergent forms of accessing, distributing, and using information to articulate and display dissent. Digitally networked information has, thus, become an instrument against authorities and power structures.

Whistleblowing-which existed long before the Internet-is one prominent phenomena that underpins the importance of understanding information power dynamics. The action itself does not necessarily depend on technology; however, ICTs contributed to the professionalisation of the act of whistleblowing, lowering the risks and developing new modes of action, such as online publishing technology, and security and privacy technologies (Heemsbergen, 2013: 67). Similar to the use of DDoS software tools to facilitate protest actions, the technical components of whistleblowing now frequently depend on specific services, which have become part of the action. Both technology and human action are shaping the political act.

Specifically since the case of Edward Snowden and his revelations about the US National Security Agency (NSA) global mass surveillance in 2012, whistleblowing as a political practice has become widely known and discussed. Yet, despite having been a common practice for centuries, the concept itself is still 'far from having a settled definition' (Davis, 2005). Different types of whistleblowing exist, and not all of them are necessarily a political action. Instead, they could potentially be motivated by personal interest and non-political gains.

In spite of this, the current paper conceives of cases of whistleblowing as forms of civil 
disobedience when an element of political motivation is evident. We thereby refer to Kumar (2013), who discerns six inherent elements of whistleblowing: (a) it is a deliberate act; (b) it is often done by an insider having access to information and an expertise in assessing the information; (c) the information is directly related to threats to citizens' rights, their obligations or harm the public interest; (d) it is assumed by the whistleblower that withdrawing such information from the public is a grave wrong done to the citizens; (e) the information is such that the public ought to know, and (f) it is in the form of an appeal to the higher authorities, through publicity, with an intention to generate public pressure to correct the wrongs done (Kumar, 2013: 129f).

Kumar, who addresses the question if and under which conditions cases of political whistleblowing can be understood as civil disobedience, concludes that actions of whistleblowers meet civil disobedience as a form of epistemic disobedience when:

[t]he disobedient fulfils his [or her] moral duty by exposing the informational asymmetry that protects the wrong-doers, and the democratic deficit within the institution. In doing so the disobedient moves beyond narrow constraints of legal duty, which binds her to the oath of secrecy, to fulfil their obligation to the citizens (Kumar, 2013: 29).

Hence, the public is the main addressee of the information made transparent and numerous whistleblower platforms like WikiLeaks.org, Publeaks.nl, and Globaleaks.org describe their works as a fight against the threat of information asymmetry to citizens' rights. It is a peculiarity of the information society that this sort of epistemic disobedience changes the ground of what we can know and thereby links the transparency of political information to political power. From a theoretical point of view, whistleblowing is thus an example of how the relationship between publicity and secrecy changes power structures.

Despite these positive elements, whistleblowing can be a highly controversial strategy and it is a risky endeavour for many actors who are involved or affected. This again emphasises the friction around the topic of (digital) civil disobedience. One substantial critique against political whistleblowing is the lack of democratic legitimacy and transparency of decisions being made by a few actors (Lovink and Riemens, 2014; Sagar, 2011). Besides problems around the external transparency of whistleblowing platforms concerning their own processes, whistleblowers or whistleblowing platforms themselves are facing constant threats from legal and state authorities. 
Notwithstanding these difficulties, numerous countries are offering increasing protection for whistleblowers as shown in the course of a comparative study of twenty countries (Wolfe et. al, 2014). Although these are favourable developments, frictions certainly remain in everyday practices such as social sanctions or job loss (Martin, 2003: 119). The power of and over information is therefore an essential element of current societal dynamics while the rising awareness that 'whistleblowing is a necessary modality of democratic self-correction' (Kumar, 2013: 126) will hopefully emphasise the political significance of whistleblowing and its relevant position as a pillar of civil disobedience in the information society.

\section{Conclusion: All Things New for Civil Disobedience?}

This paper sought to investigate a new diversity of approaches, objectives and articulations of civil disobedience. We aimed at examining the frictions existent between traditional forms of this specific act of protest and its new technological adaptations, as well as the frictions existent on the conceptual level of civil disobedience. This was done in the course of three specific sections which centred on the idea that transformations of civil disobedience do not necessarily remove legitimacy of digital civil disobedience, but demand a reconsideration of traditional understandings of civil disobedience to meet the requirements of our current society.

Thus, in the course of the first part of this paper the historical dimension of digital civil disobedience was outlined as a background for the seven factors that represent some of the features of contemporary forms of digital civil disobedience. The paper argued for a deeper assessment of these factors while acknowledging the uniqueness of each political context. These observations led to the understanding that current acts of civil disobedience are often centered around an issue of power of information, which is exemplified through the case of whistleblowing.

From a theoretical perspective, digital forms of civil disobedience extend the widespread (and often liberal) understanding of civil disobedience in a multitude of ways. One major transformation is not caused but exemplified by digital acts of civil disobedience. Hence, while in most dominant philosophical theories civil disobedience is presented as a dialectical action between a citizen and the state (Allen, 2011: 133), civil disobedience in the present day increasingly emerges beyond the state level. In addition, civil disobedience addresses increasingly private or international actors instead of concentrating on governmental decisions or institutions (Bentouhami, 2007). 
These and other challenges to existing theory lead to a process of rethinking civil disobedience in political philosophy that explores civil disobedience beyond the state paradigm (Allen, 2011; Cabrera, 2011). This is often described in terms of transnational and transversal civil disobedience (Bentouhami, 2007). As possibilities for worldwide surveillance enabled by the architecture of the Internet create new reasons for protest, the same architecture facilitates a new level of global inter-connective action that does not evolve within certain national borders. Rather it assembles around globally shared issues, whereby digital tactics of civil disobedience both enhance and enable this form of transnational dissent. We are also keen to emphasise that our paper is addressing a theoretical debate rather than aiming for empirical generalisations. Further theoretical, but certainly also empirical research is needed to examine these novel factors and frictions of digital dissent.

In conclusion, civil disobedience is not a label that can be attached to a practice or actor in general. Civil disobedience does not come with legitimacy* per se*. Stories of civil disobedience tend to be told as either stories of heroes or radical outlaws, depending on the interlocutor. To be sure, both approaches have the ability to offer a compelling story, but hero or villain narratives have their downsides, in that they falsely lead us to believe that the protagonists are either superhuman or inhumane. Both narratives conceal the fact that the disobedient individuals are people like any other, and that their actions come with risks and challenges. They are neither morally superior nor necessarily invincible, neither holy nor inviolable. Still, they often perform a very crucial task in society: they voice their concerns, disrupt routines, and demand that others listen and take action for change, which, regardless of whether one agrees or disagrees, deserves attention.

\section{Author Biographies}

Theresa Züger is currently a PhD researcher at the Humboldt Institute for Internet and Society. In her PhD project and publications, she focuses on the topic of 'digital civil disobedience' and aims to rethink the traditional concept of civil disobedience for the digital age from the perspective of political theory.

Stefania Milan (stefaniamilan.net) is curious about the intersection of technology, activism, and cyberspace governance. Currently, she is Assistant Professor of New Media at the University of Amsterdam, where she works on the politics of big data. She is the author of Social Movements and Their Technologies: Wiring Social Change (Palgrave Macmillan, 2013) and co-author of Media/Society (Sage, 2011). 
Leonie Maria Tanczer is PhD Candidate at the School of Politics, International Studies and Philosophy, Queen's University Belfast. Her interdisciplinary research includes input from both the social sciences and engineering and investigates emerging cyber security governance strategies, with a particular focus on hacking and hacktivism. Further research interests include Gender Studies, online collective action, and Science and Technology Studies.

\section{Notes}

[1] Certainly not all actions of Anonymous have a connection to civil disobedience. However, their activism raises relevant questions about means of resistance and collective self-determination in the information society.

[2] Tor (https://www.torproject.org/) is an open source software that enables anonymity and counteracts surveillance.

\section{References}

Allen, Michael. 'Civil Disobedience, International' in Encyclopedia of Global Justice (New York: Springer, 2011).

Arendt, Hannah. 'Civil Disobedience' in Crises of the Republic: Lying in Politics, Civil Disobedience on Violence, Thoughts on Politics, and Revolution (New York: Harvest* Books, 1972): 49-102. Auty, Caroline. 'Political Hacktivism: Tool of the Underdog or Scourge of Cyberspace?', Aslib Proceedings* 56 (2004): 212-221.

Bang Lab and Electronic Disturbance Theater, 2.0. 'Transborder Immigrant Tool A Mexico/ U.S Border Disturbance Art Project', (n.d.) http://bang.transreal.org/transborder-immigranttool/

Barlow, John Perry. 'A Declaration of the Independence of Cyberspace***(1996) https:// projects.eff.org/ ${ }^{\sim}$ barlow/Declaration-Final.html

Bazzichelli, Tatiana. Networking. The Net as Artwork (Aarhus: Aarhus University, 2008).

Benkler, Yochai. 'WikiLeaks and the Networked Fourth Estate', in Benedetta Brevini, Arne Hintz and Patrick McCurdy (eds) Beyond WikiLeaks: Implications for the Future of Communications, Journalism and Society (Basingstoke: Palgrave Macmillan, 2013): 11-34. 
Benkler, Yochai, Hal Roberts, Robert Faris, Alicia Solow-Niederman and Bruce Etling. 'Social Mobilization and the Networked Public Sphere: Mapping the SOPA-PIPA Debate', Berkman Center Research Publication 16 (2013): 1-54. http://papers.ssrn.com/sol3/papers. cfm?abstract_id=2295953

Bennett, Lance and Alexandra Segerberg. 'The Logic of Connective Action: Digital Media and the Personalization of Contentious Politics', Information, Communication \& Society 15 (2012): 739-768.

Bentouhami, Hourya. 'Civil Disobedience From Thoreau to Transnational Mobilizations: The Global Challenge', Essays in Philosophy. A Biannual Journal 8.3 (2007): 1-11. http:// commons.pacificu.edu/eip/vol8/iss $2 / 3 /$

Boler, Megan. Digital Media and Democracy: Tactics in Hard Times (Cambridge: MIT Press, 2008).

Bratich, Jack. 'The Rise of Flashpublics-My Little Kony', Counterpunch (2012) http://www. counterpunch.org/2012/03/13/my-little-kony/

Brownlee, Kimberley. 'The Communicative Aspects of Civil Disobedience and Lawful Punishment', Criminal Law and Philosophy *1.2 (2007): 179-192.

Brownlee, Kimberley. Conscience and Conviction: The Case for Civil Disobedience (Oxford: Oxford University Press, 2012).

Bunz, Mercedes. Die Stille Revolution: Wie Algorithmen Wissen, Arbeit, Öffentlichkeit Und Politik Verändern, Ohne Dabei Viel Lärm Zu Machen (Berlin: Suhrkamp, 2012).*

Castells, Manuel. The Rise of the Network Society, The Information Age: Economy, Society and Culture Vol. I. (Oxford: Blackwell, 2000).*

Cabrera, Luis. "Why Can't We Have That? "Global Civil Disobedience" and the European Living Laboratory', Open Democracy *(2011), https://www.opendemocracy.net/luis-cabrera/ why-can\%E2\%80\%99t-we-have-that-\%E2\%80\%98global-civil-disobedience\%E2\%80\%99and-european-living-laboratory

Calderaro, Andrea and Anastasia Kavada. 'Special Issue on Online Collective Action and Policy Change', Policy \& Internet 5.1 (2013): 1-6.

Celikates, Robin. 'Ziviler Ungehorsam Und Radikale Demokratie', in Thomas Bedorf and Kurt Röttgers (eds.) Das Politische Und Die Politik (Berlin: Suhrkamp, 2010): 274-300.

Celikates, Robin. 'Civil Disobedience as A Practice of Civic Freedom', in James Tully (ed.) On Global Citizenship. James Tully in Dialogue (London: Bloomsbury Press, 2014): 207228.

Celikates, Robin. 'Civil Disobedience' in Gianpietro Mazzoleni (ed.). The International Encyclopedia of Political Communication (Malden: Wiley-Blackwell, forthcoming).

Coleman, Gabriella. 'Geeks are the New Guardians of Our Civil Liberties', Technology Review (2013), http://www.technologyreview.com/news/510641/geeks-are-thenew- 
guardians-of-our-civil-liberties/

Coleman, Gabriella. Hacker, Hoaxer, Whistleblower, Spy: The Many Faces of Anonymous (New York: Verso, 2014).

Critical Arts Ensemble. Electronic Civil Disobedience (New York: Autonomedia, 1996).

Critical Arts Ensemble. Digital Resistance: Explorations in Tactical Media. (New York: Autonomedia, 2001).

Colby, Hall. 'Anonymous" Posts Video Describing "Operation Payback" In Defense Of WikiLeaks', Mediaite (2010), http://www.mediaite.com/online/anonymous-posts-videomessage-describing-their-mission-in-defense-of-wikileaks/

Danyel, Jürgen. 'Zeitgeschichte der Informationsgesellschaft', Zeithistorische Forschungen/ Studies in Contemporary History 9.2 (2012): 186-211.

Davenport, David. 'Anonymity on the Internet: Why the Price May Be Too High', Communications of the ACM 45.4 (2002): 33-35.

Davis, Michael. 'Whistleblowing', in Hugh LaFollette (ed.)* The Oxford Handbook of Practical Ethics *(Oxford: Oxford University Press, 2009): 539-566.

Deitz, Milissa. 'Cut and Paste: Australia's Original Culture Jammers, BUGA UP.'* Global Media Journal: Australian Edition *8.1 (2014): 1-10.

Denker, Kai. 'Heroes Yet Criminals of the German Computer Revolution' in Gerard Alberts and Ruth Oldenziel (eds.) Hacking Europe: From Computer Cultures to Demoscenes (London: Springer, 2014): 167-187.

Dominguez, Ricardo. 'A Transparent and Civil Act of Disobedience', The Thing (2005) http:// post.thing.net/node/304

Dunn Cavelty, Myriam and Mark Daniel Jaeger. '(In)visible Ghosts in the Machine and the Powers that Bind: The Relational Securitization of Anonymous', International Political Sociology (forthcoming).

Dworkin, Ronald. 'Civil Disobedience and Nuclear Protest' in Ronald Dworkin (ed.). A Matter of Principle (Cambridge: Harvard University Press, 1985): 104-117.

Franklin, lain. 'Saving Face - The Facts About Website Vandalism', Computer Fraud \& Security 2 (2001): 8-18.

Foredyke, Robbie. 'DDoS Attacks As Political Assemblages', Platform. Journal of Media and Communication 5.1 (2013): 6-20.

Galloway, Alexander and Eugene Thacker. The Exploit: A Theory of Networks (Minneapolis and London: University of Minnesota Press, 2007).

Garfield, Bob. 'The Many Moods of Anonymous', On The Media (2011), http://www. onthemedia.org/story/133097-the-many-moods-of-anonymous/transcript/ 
George, Cherian. 'The Internet As A Platform for Civil Disobedience' in John Hartley, Jean Burgess and Axel Bruns (eds).* A Companion to New Media Dynamics *(Malden: WileyBlackwell, 2013), 385-395.

Gibbs, Samuel. 'Angry Birds site defaced with 'spying birds' spoof after NSA revelation', The Guardian (2014), http://www.theguardian.com/technology/2014/jan/30/angry-birdsdefaced-nsa-spying-birds-user-data

Gimmler, Antje. 'Deliberative democracy, the public sphere and the Internet', Philosophy \& Social Criticism 27.4 (2001): 21-39.

Habermas, Jürgen. 'Civil Disobedience: Litmus Test for the Democratic Constitutional State' Berkeley Journal of Sociology 30**(1985): 95-116.

Hahn, Henning. 'Ungehorsam, Ziviler', in Stefan Gosepath, Wilfried Hinsch and Robin Celikates, Wulf Kellerwessel (eds). Handbuch der politischen Philosophie und Sozialphilosophie (Berlin: De Gruyter, 2008): 1365-1366.

Halupka, Max. 'Clicktivism: A Systematic Heuristic', ${ }^{*}$ Policy \& Internet *6. 2 (2014): 115-132.

Hands, Joss. @ Is for Activism: Dissent, Resistance and Rebellion in a Digital Culture (London: Pluto, 2011).

Heemsbergen, Luke. 'Whistleblowing and Digital Technologies: An Interview with Suelette Dreyfus', PLATFORM Journal of Media and Communication 5.1 (2013): 67-71.

Hentschel, Ulrike. 'Das Theater als moralisch-pädagogische Anstalt? Zum Wandel der Legitimationen von der Pädagogik des Theaters zur Theaterpädagogik' in Eckart Liebau, Leopold Klepacki, Dieter Linck, Andreas Schröer and Jörg Zirfas (eds). Grundrisse des Schultheaters. Pädagogische und ästhetische Grundlegung des Darstellenden Spiels in der Schule (München: Beltz Juventa, 2005): 31-52.

Jordan, Tim and Paul A. Taylor. Hacktivism and Cyberwars. Rebels with a Cause? (London: Routledge, 2004).

Kalyvas, Andreas. Democracy and the Politics of the Extraordinary: Max Weber, Carl Schmitt, and Hannah Arendt. (Cambridge: Cambridge University Press, 2008).

Klang, Mathias. 'Civil Disobedience Online', Journal of Information, Communication and Ethics 2.2 (2008): 75-83.

Kumar, Mandhar. 'For Whom the Whistle Blows? Secrecy, Civil Disobedience, and Democratic Accountability' PhD Thesis, LUISS Guido Carli.

Laudani, Raffaele. Disobedience in Western Political Thought: A Genealogy (New York, Cambridge University Press, 2013).

Lovink, Geert and Patrice Riemens. 'Twelve Theses on WikiLeaks', in Benedetta Brevini, Arne Hintz and Patrick McCurdy (eds.) Beyond WikiLeaks: Implications for the Future of Communications, Journalism and Society (Basingstoke: Palgrave Macmillan, 2013): 245253. 
Mansfield-Devine, Steve. 'Hacktivism: Assessing the Damage', Network Security 8 (2011): 5-13.

Martin, Brian. 'Illusions of Whistleblower Protection', University of Technology Sydney Law Review 5 (2003): 119-130.

Mazzoleni, Gianpietro and Winfrid Schulz. “Mediatization” of Politics: A Challenge for Democracy? Political Communication', Political Communication 16.3 (1999): 247-261.

Milan, Stefania. 'Hacktivism As a Radical Media Practice', in Chris Atton (ed.). Routledge Companion to Alternative and Community Media (London: Routledge, 2015): 550-560.

Milan, Stefania. Social Movements and Their Technologies: Wiring Social Change.

(Basingstoke: Palgrave Macmillan, 2013a).

Milan, Stefania. 'WikiLeaks, Anonymous, and the exercise of individuality: Protesting in the cloud', in Benedetta Brevini, Arne Hintz and Patrick McCurdy (eds). Beyond WikiLeaks: Implications for the Future of Communications, Journalism and Society (Basingstoke: Palgrave Macmillan, 2013b), 191-208.

Morozov, Evgeny. The Net Delusion: How Not to Liberate the World (London:* *Penguin, 2011).

Münker, Stefan. Emergenz Digitaler Öffentlichkeiten (Frankfurt: Suhrkamp, 2012).

Nadir, Leila. Poetry, Immigration and the FBI: The Transborder Immigrant Tool.

Hyperallergic (2012)http://hyperallergic.com/54678/poetry-immigration-and-the-fbi-thetransborder-immigrant-tool/

NATO. 'Information and National Security. Draft General Report to the NATO Parliamentary Assembly by Lord Jopling (United Kingdom), General Rapporteur', NATO, (2010), http:// www.nato-pa.int/default.asp?SHORTCUT=2443

Nazario, Jose. 'DDoS Attack Evolution',' Network Security 7 *(2008): 7-10.

Paris, Jeffrey and Michael Ault. 'Subcultures and Political Resistance', Peace Review: A Journal of Social Justice 16.4 (2004): 403-407.

Postmes, Tom and Suzanne Brunsting. 'Collective Action in the Age of the Internet Mass Communication and Online Mobilization', Social Science Computer Review 20.3 (2002): 290-301.

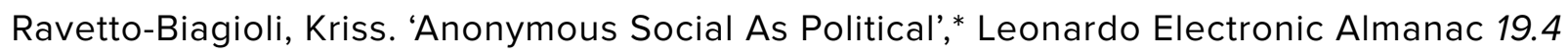
*(2013): 178-196.

Rawls, John. A Theory of Justice (Oxford: Clarendon Press, 1972).

Raz, Joseph. The Authority of Law: Essays on Law and Morality (Oxford: Oxford University Press, 1994): 262-275.

Rotman, Dana, Sarah Vieweg, Sarita Yardi, Ed Chi, Jenny Preece, Ben Shneiderman, Peter 
Pirolli, Tom Glaisyer.'From Slacktivism to Activism: Participatory Culture in the Age of Social Media', Proceedings of the CHI '11 Extended Abstracts on Human Factors in Computing Systems (2011): 819-22.

Sagar, Rahul. 'Das missbrauchte Staatsgeheimnis' in Geiselberger, Heinrich (ed.). Wikileaks und die Folgen: Netz - Medien - Politik (Berlin: Suhrkamp 2011): 201-223.

Sauter, Molly. 'Distributed Denial of Service Actions and the Challenge of Civil Disobedience on the Internet***Master Thesis, Center for Civic Media, MIT.

Sauter, Molly. The Coming Swarm: DDOS Actions, Hacktivism, and Civil Disobedience on the Internet (New York and London: Bloomsbury, 2014).

Schwarz, Elke. '@ Hannah_arendt: An Arendtian Critique of Online Social Networks', Millennium-Journal of International Studies 43 (2014): 165-186.

Shantz, Jeff and Jordon Tomblin. Cyber Disobedience: RE: //Presenting Online Anarchy (Winchester: Zero Books, 2014).

Sifry, Micah. The Big Disconnect: Why the Internet Hasn't Transformed Politics (Yet) (New York: OR Books, 2014).

Strano Network and Tommaso Tozzi. Net Strike, No Copyright, ecc. (Bertiolo:* *AAA Edizioni, 1996).

Stöcker, Christian. 'Facebook-Spam: Russland-Freunde aus der rechten Ecke', Spiegel Online (2014), http://www.spiegel.de/netzwelt/netzpolitik/facebook-spam-beideutschenmedien-unter-namen-anonymous-a-964869.html

Tactical Media. 'Electronic Civil Disobedience, Simulation, and the Public Sphere', Tactical Media Files (n.d.), http://www.tacticalmediafiles.net/article.jsp?objectnumber=42809

Tanczer, Leonie. Hacking the Label: Hacktivism, Race, and Gender. Ada: A Journal of Gender New Media and Technology, 6 (2015) http://adanewmedia.org/2015/01/issue6tanczer/ Thoreau, Henry David. Civil Disobedience, and Other Essays (New York: Dover Publications, 1993).

Tsing, Anna Lowenhaupt. Friction: An Ethnography of Global Connection (Princeton N.J.: Princeton University Press, 2005).

Tozzi, Tommaso. 'Netstrike', Netstrike (1995) http://www.tommasotozzi.it/index. php?title=Netstrike_\%281995\%29

Volk, Cristian. 'Herrschaft in der Internationalen Politik-Widerstand zwischen Entpolitisierung und Radikalisierung', in Julian Junk and Christian Volk (eds.) Macht und Widerstand in der globalen Politik (Baden Baden: Nomos Verlag, 2013): 49-72.

Warner, Gary. 'Internet Anarchy: Anonymous Crowds Flex Their Muscles', CyberCrime \& Doing Time A Blog about Cyber Crime and Related Justice Issues, (2010), * "http:// garwarner.blogspot.co.uk/2010/12/internet-anarchy-anonymous-crowds-flex.html

Webster, Frank. Theories of the Information Society (Abingdon: Routledge, 1995). 
Wilde, Oscar. The Soul of Man Under Socialism (Portland, Me: T.B. Mosher: 1909).

Wolfe, Simon, Mark Worth, Suelette Dreyfus, and A. J. Brown. Whistleblower Protection Rules in G20 Countries: The Next Action Plan (Melbourne: Public* *Consultation Draft, 2014).

Wray, Stefan. 'On Electronic Civil Disobedience', Peace Review 11.1 (1999): 107111.

Ziccardi, Giovanni. Resistance, Liberation Technology and Human Rights in the Digital Age (Dordrecht: Springer, 2012).

Zinn, Howard. 'Law, Justice and Disobedience', Notre Dame Journal of Law, Ethics \& Public Policy 5 (1991): 899- 919.*

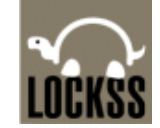

The LOCKSS System has the permission to collect, preserve and serve this open access Archival Unit

\section{OPEN HUMANITIES PRESS}

The Fibreculture Journal is published by The Fibreculture Journal Incorporated in partnership with Open Humanities Press. 


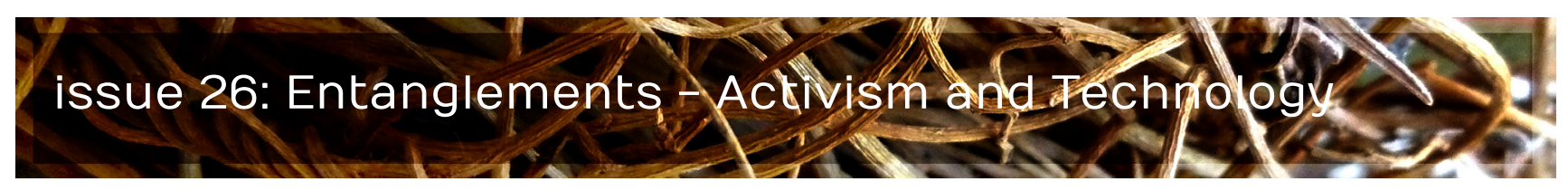

\title{
FCJ-193 Harbouring Dissent: Greek Independent and Social Media and the Antifascist Movement
}

\author{
Sky Croeser \\ Curtin University
}

Tim Highfield

Queensland University of Technology

\begin{abstract}
:
This article examines Greek activists' use of a range of communication technologies, including social media, blogs, citizen journalism sites, Web radio, and anonymous networks. Drawing on Anna Tsing's theoretical model, the article examines key frictions around digital technologies that emerged within a case study of the antifascist movement in Athens, focusing on the period around the 2013 shutdown of Athens Indymedia. Drawing on interviews with activists and analysis of online communications, including issue networks and social media activity, we find that the antifascist movement itself is created and recreated through a process of productive friction, as different groups and individuals with varying ideologies and experiences work together.
\end{abstract}

doi: $10.15307 /$ fcj.26.193.2015 


\section{Introduction}

Activists' uses of digital technologies are complex, and technologies are not only shaping the available possibilities for social change but are also being changed themselves through activists' work. In this article we look at Greek activists' use of a range of communication technologies, including social media like Twitter and Facebook, blogs, citizen journalism sites, Web radio, anonymous networks, and email. We use Anna Tsing's (2005) model of friction to understand how frictions might productively influence or slow the use of particular digital technologies, examining the intersections between human and non-human actors, ideologies and experiences, in influencing the choices made by activists.

Our analysis focuses on the Greek antifascist movement, primarily in Athens, noting that 'the antifascist movement' is largely a constructed object. We see Greek anarchist and anti-authoritarian organising as a key element of this movement, and note that E. Dimitris Kitis (2015: 2) has suggested that this be seen as a chóros (which translates to 'space', but is more accurately a 'scene' or 'milieu'), which 'replaces the notion of a specific social movement or subculture with one of a more fluid assortment of people and ideas, including one that is not even constant in nature and time'. However, we continue to use the term 'movement' here to emphasise (in line with Croeser, 2012; 2014) that no movements are as cohesive or as unitary as they are often depicted in social movement studies. Many of those we interviewed would also reject the label 'activist', and so while we use the words 'activist' and 'movement,' in doing so we attempt to problematise them. These terms are 'zones of awkward engagement, where words mean something different across a divide even as people agree to speak' (Tsing, 2005: xi). Anarchist and autonomous activists, immigrants trying to protect their communities, journalists, and others create unexpected and temporary alliances, even as differences continue to be important and, frequently, divisive. To the extent that there is such a thing as a Greek antifascist movement, it is not a pre-existing phenomenon which then uses social media in particular ways: rather, it is created and constantly recreated in new and slightly different ways through particular spaces and events, including through social media.

With these caveats in mind, we discuss this movement focusing specifically on its activities in April and May 2013, a period that saw the enforced shutdown of three independent media channels, including the Athens Indymedia site. This discussion must be put in the context of several interrelated trends affecting Greece. The economic crisis that was beginning to manifest in 2008 was accompanied by a shift towards austerity politics, leading to cuts to social support services and to the public sector, including media. At the same time, government control of public space and spaces of dissent was tightening, 
prompted in part by pressure from private interests and a strong relationship with private media. The economic crisis, bolstered by right-wing rhetoric from mainstream politics parties (particularly Nea Democratia or New Democracy) and Greek private television, exacerbated the xenophobia which had been rising in Greece since the 1990s (Psarras, 2013). These trends facilitated the growth of Chrysi Avgi, Golden Dawn, a neo-Nazi organisation officially founded in 1980 (Psarras, 2013: 6). The group, which had been largely irrelevant in previous decades, entered the Greek national parliament in 2012. Antifascist activists were therefore operating in a context where: Greek society had very high levels of racism; an openly fascist party had gained enough support to be represented in parliament; mainstream political parties were stoking the fires of xenophobia; attacks on independent media were increasing (as we discuss in more detail below); and there were signs of significant support for Golden Dawn among Greek police (Margaronis, 2012).

Pantelis Vatikiotis (2011: 172) has noted that prior to the 2008 protests (discussed below) little attention had been brought to bear on sub-cultures and social movements in the margins in Greece, and in particular there was limited work 'on grassroots media practices in Greece that have diachronically promoted the inclusion of marginalised social domains, heterogeneous discourses, and diverse social actors in public and political life.' Since 2008, work in this area has increased significantly, with extensive literature examining the 2008 protests (Karamichas, 2009; Sotiris 2010; Vradis and Dalakoglou, 2011; Kornetis, 2010; Chatzidakis, 2013; Kalyvas, 2010), and/or Greek activists' media practices (Milioni, 2009; Milioni and Panos, 2011). However, this literature has rarely examined the frictions in activists' use of different technologies - that is, the ongoing sparks and reorientations created by and through the relationships between activists' ideologies, the embedded affordances of technologies, and other actors.

The intersections between social movements and digital technologies, and the way these are changing over time, have been the subject of extensive international research across platforms and activist communities, from the Zapatista movement in Mexico in the 1990s to Occupy Wall Street and its offshoots (see, among others, Meikle, 2002; Papacharissi, 2015; Bennett and Segerberg, 2013). Tsing's model of frictions offers an opportunity to deepen this work by inviting us to consider 'the awkward, unequal, unstable, and creative qualities of interconnection across difference' (2005: 4). Although Tsing explores the model primarily in reference to the relationships between individuals and groups, friction can be extended as a useful metaphor for understanding our relationships with technology: activists' use of digital technologies involves similarly awkward, unstable, and creative connections across difference, both interpersonal and between human and non-human actors. 


\section{Leaving Exarcheia - the growth of the Athenian antifascist media ecology since 2008}

The Greek media environment has undergone significant changes since 2008 , and has been profoundly affected by austerity politics and the accompanying extension of mechanisms of social control. Early signs of the Greek impacts of the global capitalist crisis were seen in 2008, when negative growth rates in the economy were accompanied by increasing unemployment, and huge growth in poverty, social exclusion, and homelessness. The Greek government, in line with loan conditions imposed by the IMF, responded by slashing the social welfare net and cutting social services, including health services (Kondilis et al., 2013: e1). At the same time, the policing of public spaces and reports of police violence intensified (Occupied London Collective, 2011: 329; Sotiris, 2010). This combination of economic pressures and growing social controls set the scene for the events of December 2008.

Many commentators see the 2008 protests as creating a fundamental change in the Greek political landscape, and Giovanopolous and Dalakoglou have argued that, "any attempt to return to the pre-December 2008 political normalities is impossible at any level and for any actor in Greek political life' (2011: 112). The catalyst for 2008's massive protests was the murder of Alexandros Grigoropoulos, a 15-year old, by two policemen. Grigoropoulos was shot on 6 December in Exarcheia, a neighbourhood particularly associated with the anarchist movement or, as Makrygianni and Tsavdaroglou (2011: 40) describe it, "ground occupied by the antagonistic movement'. The response was swift, with mobilisation happening immediately through phone calls and the Indymedia site. One interviewee, Antonis Broumis, spoke of receiving a call from his brother, telling him about the murder, and about the failure of the mainstream media to provide useful information:

...because the mainstream media came out with a story, "we don't know who shot, maybe he still lives", when riots started everywhere the whole thing collapsed, because people knew through the Indymedia, not through the mainstream media.

The Indymedia Athens website was visited over ten million times in the days following Grigoropoulos' murder (Dalakoglou and Vradis, 2011: 18). As protests gathered force over the following days, communications over Indymedia and mobile phone networks continued to play a key role. 
Distrust of the mainstream media was exacerbated by the perceived misrepresentation of the Greek anti-austerity protests, centred on the occupation of Syntagma Square in Athens, in 2011 and 2012. Theocharis et al. argue that this was a central complaint amongst Greek protesters, and led to 'the Greek movement's explicit hostility towards mass media and to a turn towards alternative avenues for information' (2014: 210). Several interviewees noted that during the Syntagma Square protests, independent media played a key role. For example, an anonymous participant noted the importance of Radio Entasi's presence at the site of the occupation, 'between the teargas and the riot police', while another, IrateGreek, talked about the significance of the Syntagma protests in increasing use of the \#rbnews hashtag which has become central to RadioBubble's reporting (and indeed to much of the Greek news media ecology). As independent media sources expanded, they have contributed to the ongoing research and coverage of the relationship between the mainstream media and business elites (see for example, Inflammatory, 2013), many of whom have also been charged with fraud or linked to government corruption, reinforcing the distrust of mainstream media.

Both the Greek state and far-right groups have begun to respond to the growth of independent media and critical perspectives. Greek activists and commentators point to a number of moves by the Greek government that, they argue, aim to shut down dissent. IrateGreek argued that there is a pattern emerging in which the most marginalised spaces face repression first-as seen in the violent police closure of squatted social centre Villa Amalias described by Dalakoglou (2013)—with other spaces of dissent following. The shutdown and "reorganisation" of the national broadcasting station, ERT, in June of 2013 can also be seen in this light: journalists from the ERT, unions, and press freedom organisations framed this as an attack on democracy with chilling effects on media freedom (Lowen, 2013). It is frequently assumed that state attacks on spaces of dissent are closely tied to the interests of economic elites: for example, in an interview with Giorgos Kyritsis, a journalist from Avgi-a more mainstream left news publication which nevertheless sees its work as being aligned with the antifascist movement-he said, 'we have been sued many times, not from the government, from others, who happen to be friends with the government'. At the same time, Golden Dawn has been playing an active role in attacks on dissenting spaces, ranging from asking pointed questions about the location of Indymedia servers as early as 2009 (IMC, 2009) to engaging in what seem to be coordinated efforts to shut down Greek antifascist accounts on Facebook (Siapera, 2014; MatӨaíoc, 2013). Formal mechanisms of state repression combine in complex ways with other forms of control to put pressure on the growth of independent media.

The shutdown of Indymedia, Radio Entasi and 98FM needs to be understood in this context; while this is a relatively minor event in the history of the antifascist movement, it does highlight many of the broader trends affecting activists' use of digital technologies 
in Greece. The shutdown happened when servers hosting independent media sites were turned off on the order of the Dean of the National Technical University in Athens (NTUA) on 11 April 2013, but many commentators saw this as a result of pressure from the government, referencing a tweet from New Democracy MP Adonis Georgiadis

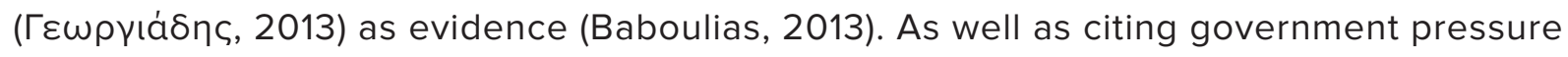
as a motivation, several interviewees noted ongoing questions from Golden Dawn in parliament and potential attempts by mining companies to undermine resistance to the Skouries mine in lerissos. As this shutdown demonstrates, activists clearly understand attacks on their communications and media as extending significantly beyond a model of state-based censorship, encompassing deeper concerns with the relationship between capitalism, state power, and communicative technologies.

\section{Research design and methodology}

This article forms part of our wider Mapping Movements project, which studies the intersections between social movements and technologies, taking in case studies from around the world: our research so far has also included fieldwork and online analysis of Occupy Oakland in the US (Croeser and Highfield, 2014) and the 2013 World Social Forum held in Tunisia. For the Greek case study, we use a mixed-methods approach combining fieldwork and interviews with activists in Athens with digital methods, drawing on hyperlink networks and Twitter data. This approach allows us to garner a more nuanced view of the movement and its relationship with online platforms than would be apparent from social media activity alone (see also approaches used by, for example, Gerbaudo, 2012). The methodology is designed as a means of overcoming some of the limitations found in social movement research, particularly around social media analysis. Our methods also respond to ethical concerns and challenges, which may be well documented by previous literature on social movements, but which have not yet received a consistent response in the case of social media studies.

We used IssueCrawler software to follow hyperlinks from a seed list of websites related to the movement (including blogs) to identify additional sites to which they are connected. The results of the crawls allow the identification of issue and solidarity networks, associated in some way with the movement (see, for example, Marres and Rogers, 2005; Bennett and Segerberg, 2013). The depth of the crawl also allows for examining whether a movement has connections to other issues and movements, at the local and international levels, or if it combines multiple issue networks (or indeed moves from a single-issue network to a wider, thematically-consistent network within which the single-issue networks are located). The value of identifying online issue networks is seen by how they highlight 
the resources, organisations, media, and other websites (including social media platforms) that are connected, cited, or invoked through hyperlinks from sites discussing a given issue. This is particularly important when considering how social movements might employ different platforms and sources over the course of a protest or campaign.

For our case study, we drew on seed lists of websites set up around the antifascist movement, and also sites covering and created in response to the specific event of the Athens Indymedia shutdown on 11 April 2013. Although Indymedia is not an antifascistspecific platform, the two contexts are linked, especially in the use of independent media by activists and the importance of these channels to movements covering a range of issues. Kriditis' (2014: 80 ) discussion of the anarchist movement in Greece, for example, positions the establishment of Indymedia Athens in 2001 in the context of the broader history of opposition to fascism that existed in Greece even before WWII and the military junta of 1967. These examinations of the social movement's online connections then demonstrate the positioning of antifascist communication within a wider media ecology, at the local and international levels. The issue networks are not without their limitations: the purpose of this step is not to create a definitive overview of the antifascist movement's (public) connections, but to identify links between different groups, locally and internationally, which can be examined further through qualitative means through fieldwork and additional digital analysis. This research is supplemented by studying tweets and websites identified from the crawls and related hashtags, such as \#rbnews and \#free_indymedia, to highlight additional practices and frictions, and to further develop our analysis of the intersections between social movements and digital media.

Fieldwork and interviews for the project were carried out by Croeser and took place over five weeks in April and May 2013. This period serendipitously overlapped with events which, while by no means the most central event in the movement's history, offer a prism through which to view some of the frictions in Greek antifascist activists' use of different communicative technologies: the temporary shutdown of Athens Indymedia, Radio Entasi, and 98FM on 11 April 2013. This allowed access to debates taking place within the movement about which communicative technologies to use, how to approach and shape these technologies, and around long-term strategies for communication within the movement and with outside audiences. Fieldwork involved attendance at several meetings dealing with potential responses to the shutdown; protests around the shutdown ranging from small events (with approximately 30 participants) to a larger march (attended by approximately 4,000 people); and participation in a range of movement spaces, including radio stations and social centres. This provided valuable insights into discussions around activists' use of different technologies. 
In addition to this, in-depth, semi-structured interviews were carried out with 34 people associated with the movement, with attempts made to gather a range of perspectives. Snowball sampling with multiple starting points was an important factor in selecting participants, both as a way of triangulating participants considered to have useful insight into the movement and because personal introductions played an important role in convincing activists experiencing significant repression and surveillance to participate. Interviewees included those deeply involved in the antifascist movement who saw their involvement in independent media as part of this work; journalists working within mainstream media covering the rise of the far-right and nationalist political party Golden Dawn; people leading or working within immigrant groups; and those who saw themselves primarily as citizen or independent journalists. Together, these interviews allowed us to build a multi-layered perspective the movement, sharing commonalities with Tsing's use of a methodology that explores multiple viewpoints and is, 'variously ethnographic, journalistic, and archival ... formed in discrete patches' (2005: $\mathrm{x}$ ). Like Tsing, we are interested in 'odd connections rather than seamless generalizations, inclusive tables, or comparative grids.' (2005: xi).

Participants chose whether to be anonymous, or be identified by their legal names or a pseudonym. While offering all participants the option of anonymity is vital to ethical research, it is also important to recognise that some participants have compelling reasons to prefer being identified by name, or by a well-known pseudonym. One participant, IrateGreek, spoke of the protective value of visibility, knowing that the state would leave her alone because she has an international audience on Twitter. If we are to take seriously the injunctions to treat activists as those with the most valuable perspective on their own lives and work (Chesters, 2012: 145), we also need to respect their evaluations of the value and risk of public identification. Similarly, we have chosen our focus with care, particularly given that during the period of writing and research Greek media-both mainstream and independent-were increasingly under pressure from a range of directions; attacks on press freedoms in Greece were beginning to raise international concern well before our research was carried out (Borger, 2012). Like Tsing (2005: xii), we 'made choices about the kinds of research topics that seem appropriate, and, indeed, useful' based on our collaboration with activists. These, and other ethical issues related to our research methodology, are discussed in more detail in Croeser and Highfield (2015). 


\section{Frictions that slow, frictions that spark: activists' values and technological affordances}

The particular form of the Greek antifascist media ecology is shaped in part by the frictions that exist between activists' values and the embedded affordances of existing digital technologies. While, as we note above, the movement contains a wide range of politics, we have focused particularly on the work of anarchist and autonomist activists, who have been at the forefront of antifascist activism throughout recent Greek history (Kriditis, 2014; Kitis, 2015). The politics of these activists mean that they tend to value creating change on the ground rather than appealing to the government or other actors to change policy; autonomy and decentralisation; and an opposition to capitalism. These values shape, through friction, how different media are used. In particular, they lead to a significant presence for highly-localised websites linked to particular spaces within Athens (especially stekia * *'hangouts' or social centres), and extensive use of postering, which should be considered part of the media ecology in the same way that Twitter, Facebook, and Web radio are.

In addition to this, recent changes to antifascist communication practices are strongly tied to the shift away from Exarcheia, the anarchist and autonomous neighbourhood which has been the focal point for much of the Greek resistance to the right wing for decades. Now, some activists are working to decentralise the movement, shifting from Exarcheia towards neighbourhood assemblies, squats, and stekia around Athens. Squats, where local, disused spaces are occupied by activists, remain limited as a result of police repression and the dangers of attacks from fascist groups; this friction between activists and the state means that stekia, which are rented or otherwise legally acquired, play an important role in local organising. An activist from a social centre in the Zografou neighbourhood, Alex P, said in an interview, 'even us who were already involved in radical politics saw a lot of other chances to originate struggles, to create radical communities in neighbourhoods ... a lot of great things started happening in the neighbourhoods of Athens', which has been bringing new people to the movement. He said that, 'as the scene grew bigger the need for more communication and coordination was also getting bigger'. However, the choice of technologies for communication and organising is not always clear-cut.

The various digital platforms adopted by activists, and used for related topical coverage and political groups, form a digital media ecology of overlapping practices and local and international intersections. We used issue crawls to provide overviews of activist media networks originating from Greek sites, with one such network featured in Figure 1, arising from the seed list of antifascist websites. Here, independent media (including 
Athens Indymedia) and blogs receive links, as do social media and content-sharing platforms (Twitter, YouTube). What is also highlighted by the visualised networks are the connections between local and international groups and issues, with Figure 1, for instance, also featuring online resources dedicated to campaigns and themes around immigration elsewhere in Europe (such as the Calais Migrant Solidarity blog). Additional crawls, not depicted here, further emphasised the importance of location for online media, with blogs and online radio stations centred on specific parts of Athens being notable resources within the issue networks.

Figure 1, like the other issue networks identified for this project, is not without its limits; the crawls are not descriptive of the entire set of resources which are used by Greek activists, especially since not all activists are online, and communication also makes use of private

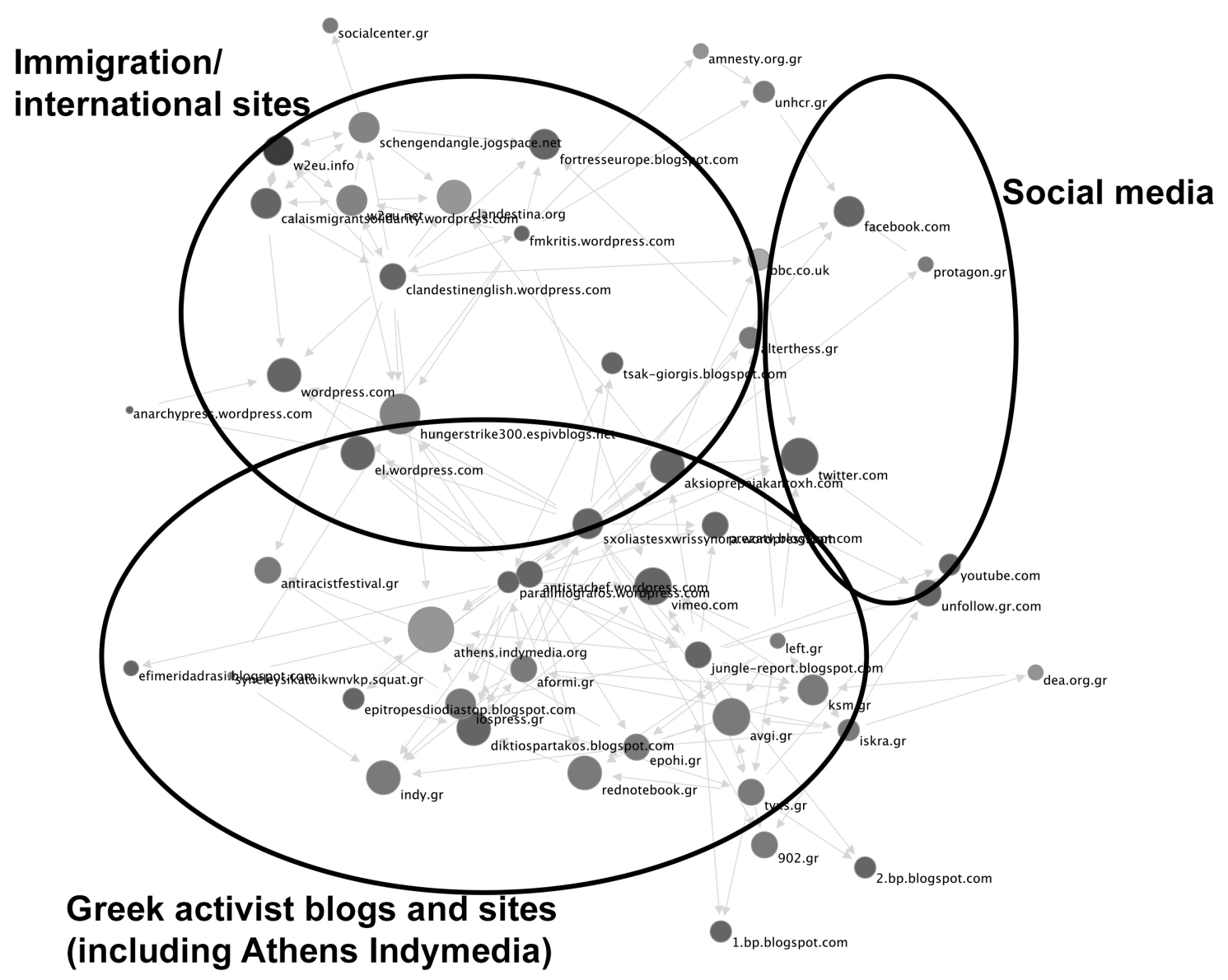

Figure 1: Antifascist issue network (crawl depth '2'), identified using IssueCrawler. [1] 
channels. Rather, the issue networks provide a visual aid for noting connections between sites and topics, which can be explored in further analysis using digital methods and through fieldwork. In Figure 1, for instance, among blogs hosted on Blogger and Wordpress, there are also topical resources featured on local, deliberately independent platforms like espivblogs. These crawls were carried out in April and May 2013; returning to the activist media ecology after the crawls also highlights the continued move towards independent and non-commercial spaces for activism online. The blog of the activist ACTION newspaper (efimeridadrasi.blogspot.com) featured to the left of Figure 1, for example, was moved from Blogger in late 2013 to a new site hosted through espiv.net (efimeridadrasi.espiv.net), as part of Greek activists' ongoing resistance towards corporate and commercial platforms.

Indeed, although blogs allow activists to maintain some control over content, form, and presentation, as opposed to social media platforms like Twitter or Facebook, even here concerns remain about censorship and surveillance, particularly for commercial services such as Blogger and Wordpress. Two activists who run a blog covering antifascist issues noted in an interview that they had specifically avoided using Blogger because of concerns with blogspot.gr being covered by Greek law, and had chosen Wordpress instead. However, they had been experiencing significant issues in the period after the Indymedia shutdown when traffic to their Wordpress site increased significantly: they were considering shifting the site and trying to find a new host service, preferably based outside of Europe and the US. Another anonymous participant argued that the online tools used by the movement:

...are structures for the movement, have been built by people from the movement, independent servers, and in a way, actually they are protecting data, and this is important for people who use those media, because other structures like Google and Wordpress are easily controlled by authorities.

Citizen-led platforms and initiatives, such as open radio and citizen journalism, give activists channels for their voices to be distinct and autonomous, particularly in response to distrust of the mainstream media. They also allow for these voices to potentially spread further, through sites such as RadioBubble where content is uploaded and shared. These platforms do not operate in isolation, but are closely interlinked, as seen in the physical connections (including using the same university space prior to the shutdown) and digital links between Athens Indymedia and 98FM. While the issue network visualisations do not in themselves explain why there are connections between sites, they do demonstrate the mix of commercial and independent platforms, and topical and geographic variations present around an initial thematic context such as the antifascist movement. These are in a state of flux, though: while commercial platforms like Twitter and Facebook offer 
a large-scale audience and centralised mechanisms for publicly sharing material, for instance, the risks and shortcomings around surveillance, privacy, and control of data and content, mean that movements are negotiating using these spaces while also promoting more independent options.

However, much of the existing literature takes it for granted that digital technologies mirror activists' values and priorities rather than questioning this process of negotiation. As a typical example, Kostis Kornetis (2010: 185) writes in his analysis of the 2008 protests that, 'Technology not only served as a vehicle of communication and co-ordination but also as the embodiment of the very political and organizational goals of the activists', combining discussion of independent and commercial media. Our research emphasises, in contrast, that activists' use of commercial and independent media is shaped by different frictions, some of which slow the use of particular technologies, while others lead to productive efforts to build alternatives.

Commercial platforms help in spreading information, promoting campaigns, and building the movement. Two activists interviewed noted that as well as hosting their site on a commercial blogging platform, many of their readers found them on Facebook, Twitter, and, somewhat surprisingly, Pinterest. Another, Persefoni, spoke about how Twitter has become a space in which people show support for and develop an identification with her local antifascist group:

...there was this guy who was saying, even though he has never showed up at our meetings, he said "ok, I am a member of anti-fascist group of [this neighbourhood], you have to go there," even though he wasn't a part of or joining our meetings, he felt he was a citizen of [the neighbourhood], but, you know, he felt that with his action, retweeting and tweeting, he would be a part of it ... he felt that he was contributing in a way.

This is in line with the significant body of literature which emphasises the value of Facebook, Twitter, and other commercial media to activists, while acknowledging the contestations and provocations within social media discussions (see, for example, Costanza-Chock, 2012; Tufekci and Wilson, 2012; Tufekci, 2013; Loza, 2014; Bonilla and Rosa, 2015).

At the same time, activists remain hesitant about these platforms. There is a broad recognition within the literature on activists' use of online communications that censorship 
and surveillance create friction, slowing or completely stopping the use of particular technologies (for more on this, see Croeser, 2012; Croeser and Highfield, 2014). The suspicions towards Twitter were reinforced in the fieldwork carried out for this study, and one of the results of authorities following social media activity in previous protests is that Greek activists are less willing to use popular commercial social media. One anonymous participant said:

...social media are not actually a free place, you know about this. Even authorities are checking what is published there, but they can be used in a radical way sometimes, and this happened in Libya, it's happened in Egypt, it's happened a lot, but in Greece it hasn't happened since [the 2008 protests].

While platforms such as Twitter can be used as tools for information dissemination and organisation by social movements, and attract attention from international audiences, Greek activists have proved resistant to fully adopting these popular social media, combining the use of these platforms with more independent sites. These practices highlight the negotiation of the need for public information-sharing and intra-movement communication and a desire to not be surveilled or monitored, leading to a productive friction concerning the types of technologies and their scopes for the movement.

However, there are also other frictions that emerge from activists' use of social media, including an ideological discomfort with the embedded values of commercial platforms. As Tsamou, involved in a group providing webmail and hosting services to activists, said, 'in Greece, people are very, let's say, hardcore for some things ... We don't want companies, we don't want sponsors or anything, we want our own infrastructure', relating this to the DIY trend in music which Kitis (2015) has cited as a key part of Greek anarchist and anti-authoritarian chóros. Censorship and surveillance of online spaces create friction that slows activists' use of particular platforms, but the commercial nature of these platforms is, perhaps, a greater source of friction for many antifascist activists who see capitalism and fascism as intertwined. While this kind of anti-capitalist resistance to using popular social media has received some attention in the literature (this is explicitly addressed in Neumayer and Valtysson, 2013:15 and implicit in much of the work on independent Web media, including Dunbar-Hester, 2009; 2012; 2014), more work is required to understand how activists negotiate the frictions between their own values and those embedded in social media.

The commitment to a DIY approach means that the Greek movement makes extensive use of older means of communicating online, with blogs, particularly independently-hosted 
blogs, and citizen journalism platforms enabling longer-form posting and more control over content, and Web radio all part of its communications. The popularity of blogs may in part have been encouraged by the actions of 2008, and the widespread critique of mainstream media associated with that period of protest: Mike Mowbray's (2010: 6) examinations of the 2008 Greek protests noted that 'it seems that each protest occupation in Greece had its own blog'. Blogs have remained vital and useful for Greek activists, especially given the comparatively more distributed nature of blogs as a preferred option over singular, corporate-run social media platforms like Twitter. This pattern is reflected in the issue networks identified for this project, where blogs were represented from a variety of hosting services and personal domains, using the major US services like Wordpress and Blogger but also Greek independent options like espivblogs.

These negotiations among Greek activists highlight how friction becomes a motivation for the adoption of new technologies (which create their own friction). The Indymedia shutdown encouraged activists to use increasingly independent and private channels for operating the site. In the immediate aftermath of the shutdown, the anonymity-focused network Tor, and its associated browser, were used to run and access Athens Indymedia. Tor's infrastructure involves the distribution and encrypted routing of web traffic to avoid surveillance and monitoring, concealing user information. However, the additional knowledge required to access Tor, and websites operated through it, made the transition less straightforward than simply changing social media or blog platforms. To this end, the indymedia.squat.gr site acted not as a mirror for the original Indymedia site, but as a how-to for activists (and other visitors) in the use of Tor to access Athens Indymedia. This information was also promoted on Twitter and on blogs, linking to the indymedia. squat.gr site and providing public details about how to access the less-public Indymedia. Providing clear, visible instructions may appear counter-intuitive, especially since the same opponents to activists might also make use of this information-although simply accessing the site through Tor is a very different proposition to attempting to shut down the Tor version. The squat website also confirmed that this approach was a very necessary and worthy step taken, as a means of continuing to support freedom of expression, counterinformation, and collective self-defence in response to state-led repression towards social movements (mammeloukos, 2013). Friction, in this case, was not simply the result of external oppression, but was also used creatively by activists.

This is also seen in the movement's responses to attacks on its physical infrastructure. The movement's communications and promotional strategies combine elements of the physical and the digital, but they also move between these different forms when others are unavailable. For example, activists are increasingly shifting their servers off university property since 2011, which saw the end of the asylum laws protecting Greek campuses from police incursions. Attacks on physical infrastructure (such as computers from squats 
being seized in police raids) are increasing attention to where servers are, and to the fundamental structures underlying communication networks. The work of activists in Athens has the potential to lead other movements to more autonomous communication practices: by demonstrating both how to successfully develop their own infrastructure, and the limitations and issues of popular, hosted services (including social media), activists may be persuaded to adopt more forms of alternative and independent media.

However, activists do not always choose to route around the damage caused by censorship. The Indymedia shutdown demonstrates that friction can become a way of making broader political structures visible and sparking action. Many of those interviewed said that there were multiple options available which would keep Indymedia up and running at its original address, including raising funds to host the site elsewhere and shifting to a mirror (as they did after some time). The decision to let the main site go down, to contest the shutdown, was a way of making visible the attack on universities as a space for dissent. In choosing not to take the path of least resistance, some activists saw themselves as recommitting to the idea of the university as an asylum and more broadly refusing to cede the idea of key spaces as public.

The loss of friction also becomes undesirable for some activists when the shift to independent media corresponds to a loss of the productive 'interconnection across distance' that Tsing cites as a key aspect of activism. Dimitra Milioni and Dionysus Panos (2011: 238) note that as 'the majority of the population shapes its understanding of the world through mass-mediated texts, especially during situations represented as crises, the communicative power of oppositional voices is limited within the partial online spheres, reaching only audiences that look for them'. Shifting away from Facebook, Twitter, or other mainstream commercial platforms also means giving up the potential to use these spaces to resist right-wing discourse or to engage with non-activist audiences. One person we interviewed talked about taking part in an ongoing 'war with fascists' on Facebook, and Twitter also serves as a public platform for debate. Theocharis et al. (2014) argue that the perception of 'very high levels of flaming and trolling in the Greek Twitter sphere' may have dissuaded many from use of the platform, leaving it to more engaged users. Indeed, Theocharis et al. found that Greek activists were not supportive of the platform 'being used predominantly for political mobilization and coordination' (2014: 208). However, during this case study, activists on Twitter using the \#free_indymedia hashtag were doing so in part to combat fascist comments made by public figures such as New Democracy MP Adonis Georgiadis (who is an active Twitter user); here, tweets were not just supporting Indymedia, but actively campaigning against (and trying to get Twitter to ban) intolerant and antagonistic views put forward by a member of the government. Online spaces outside of activist-run media have become important for those wishing to disrupt or contest Golden Dawn's narrative (Angouri and Wodak, 2014), as the Internet is a vector for Golden Dawn's 
growth (Koronaiu and Sakellariou, 2013: 332). Activists frequently seek out, rather than evade, points of friction.

Similarly, there was an effort to create friction in the life of the city in the march protesting the shut down; as one activist explained:

There is an effort from people who organise demonstrations, for the demonstrations not to be separated from the city, which can happen very easily, if the demo takes part in an empty road, or in a time when there is no actual traffic. Monastiraki is ... a place that if you gather you know that at least they will know that you gathered somewhere.

Online forms of communication and media are complemented with a strong focus on physical presence, which also offers its own forms of friction. The physical and the online are closely interlinked, as we also found in our study of Occupy Oakland (Croeser and Highfield, 2014): digital media can stand in for physical places when access is prevented, and serve coordinating and organisational roles for physical demonstrations, for example. Websites, blogs, and Web radio within the Greek context were also strongly connected to the physical locations in which they were located, specific to particular parts of Athens-and, as shown by the Indymedia shutdown, dependent on these locations and their physical infrastructure. At the same time, the digital is not the only communication approach for activists; ${ }^{* *}$ *postering, for example, plays a very significant role in the antifascist movement as a way to claim space and build a neighbourhood antifascist presence.

\section{Conclusion}

The practices surrounding digital technologies, and responses to specific platforms and challenges, within the context of Greek activism highlight the various perspectives and aims influencing the choices made by activists. Drawing on Tsing's (2005) model of friction allows us to build a more complex understanding of activists' use of digital technologies. Through our case study, we identified two key aspects in which activism and social movements are shaped, maintained, and recreated by productive friction. First, our analysis demonstrates that friction is created in multiple ways through the "creative connection across difference'. This is apparent not only between activists, but also between activists and technology. Within the Greek context, the apprehension directed 
towards commercial social media platforms, for instance, as controlled and surveilled spaces is also accompanied by an acknowledgement of their importance for sharing information and connecting groups in solidarity.

Second, the case study found that communicative practices which are (relatively) frictionfree are also frequently unproductive. Despite the promotion of the independence of activist platforms, for example, shifting entirely to independent media was not necessarily the most useful choice for the movement. Friction is not always unhelpful for social movements: it can offer a creative spark, inspiring change within-and beyond-the movement. Using independent digital platforms to communicate and host sites can provide a means of combating surveillance or censorship, but this can also be seen as a possible abandoning of commercial spaces to opposing voices: fascist and extremist groups make use of popular social media too, and the antifascist movement's negotiations towards using commercial and independent platforms includes the need to engage in order to resist and campaign against publicly inflammatory voices.

The antifascist movement itself is created (and continually recreated) through a process of productive friction, as different groups and individuals with varying (and sometimes clashing) ideologies and experiences work together. Digital technologies become important sites for this friction, as well as for friction between activists, the state, and capital. While there may be paths of less resistance, they are not always the most desirable for activists, and there are no entirely frictionless strategies for using digital technologies, whether they are independent or commercial. Activists at times respond to friction by attempting to shift to a smoother pathway, but at other times friction is used as a creative spark for change within the movement, or in society more broadly.

Athens Indymedia helps to illustrate our argument about the place and role of friction within social movements. The uses of digital technologies by the activists studied here links back to Tsing's exploration of connections across difference, between human and non-human actors as well as at the interpersonal level. The movement is not solely the work of activists, but is shaped by the choices of technologies and strategies, of issues and responses. These frictions and negotiations will continue to be critical for studies of social movements and social media: the balance between public and private, independent and commercial, and ongoing debates around data retention, surveillance, and profiling, will provide further sparks for activists in their uses-or non-uses-of digital technologies as tools of communication, organisation, and dissent. 


\section{Biographical Note}

Sky Croeser is an Adjunct Research Fellow in the Department of Internet Studies at Curtin University.

Tim Highfield is a Postdoctoral Research Fellow in Media and Communication at Queensland University of Technology, and a Sessional Academic in Internet Studies at Curtin University.

\section{Acknowledgement}

The authors would like to thank all of those who consented to be interviewed for this project, including those who provided detailed feedback on the draft, and Maria Sidiropoulos for her invaluable research assistance in producing seed lists for the issue networks and interview transcription.

\section{Notes}

[1] IssueCrawler colours nodes based on the source domain, distinguishing between, for instance, .com and .gr addresses. This colouring is not necessarily helpful for our analysis, though, given the use of blogging platforms at the .com and .net level as well as .gr. Node size is based on in-degree, with sites receiving more links from others in the network appearing larger in the visualisation. The annotations provided here are loose topical descriptions and not representative of all sites within the corresponding sections of the network. 


\section{References}

Angouri, Jo and Ruth Wodak. "They Became Big in the Shadow of the Crisis": The Greek Success Story and the Rise of the Far Right', Discourse \& Society 25.4 (2014): 540-565.

Baboulias, Yiannis. 'The Greek Government are Silencing their People', Vice UK, 16 April (2013), http://www.vice.com/en_uk/read/the-greek-government-shut-down-indymedia

Bennett, W. Lance and Alexandra Segerberg. The Logic of Connective Action: Digital Media and the Personalization of Contentious Politics (New York, NY: Cambridge University Press, 2013).

Bonilla, Yarimar and Rosa, Jonathan. '\#Ferguson: Digital Protest, Hashtag Ethnography, and the Racial Politics of Social Media in the United States', American Ethnologist 42.1 (2015): 4-17.

Borger, Julian. 'Greek Journalists Warn over Press Freedom', The Guardian, 29 October* *(2012), http://www.theguardian.com/world/2012/oct/29/greek-journalists-warn-press-freedom

Chatzidakis, Andreas. 'Commodity Fights in Post-2008 Athens: Zapatistas Coffee, Kropotkinian Drinks and Fascist Rice', Ephemera: Theory \& Politics in Organization 13.2 (2013): 459-468.

Chesters, Graeme. 'Social Movements and the Ethics of Knowledge Production', Social Movement Studies 11.2 (2012): 145-160.

Costanza-Chock, Sasha. 'Mic Check! Media Cultures and the Occupy Movement', Social Movement Studies: Journal of Social, Cultural and Political Protest 11.3-4 (2012): 375-385.

Croeser, Sky. 'Contested Technologies: The Emergence of the Digital Liberties Movement', First Monday 17.8 (2012), http://firstmonday.org/ojs/index.php/fm/article/view/4162/3282

Croeser, Sky. Global Justice and the Politics of Information: The Struggle over Knowledge. (Hoboken, NJ: Routledge, 2014).

Croeser, Sky and Tim Highfield. 'Occupy Oakland and \#oo: Uses of Twitter within the Occupy movement', First Monday 19.3 (2014), http://firstmonday.org/ojs/index.php/fm/article/ view/4827

Croeser, Sky and Highfield, Tim. 'Mapping Movements-Social Movement Research and Big Data: Critiques and Alternatives', in Ganaele Langlois, Joanna Redden and Greg Elmer (eds). Compromised Data: From Social Media to Big Data (London: Bloomsbury, 2015 (in press)).

Dalakoglou, Dimitris and Antonis Vradis. 'Introduction', in Antonis Vradis and Dimitris Dalakoglou (eds). Revolt and Crisis in Greece: Between a Present Yet to Pass and a Future Still to Come (Oakland, Baltimore, Edinburgh, London \& Athens: AK Press and Occupied London, 2011), 13-25. 
Dalakoglou, Dimitris. 'Neo-Nazism and Neoliberalism: A Few Comments on Violence in Athens At the Time of Crisis', WorkingUSA 16.2 (2013): 283-292.

Dunbar-Hester, Christina. "Free the Spectrum!" Activist Encounters with Old and New Media Technology', New Media \& Society 11.1-2 (2009): 221-240.

Dunbar-Hester, Christina. 'Soldering Toward Media Democracy Technical Practice as Symbolic Value in Radio Activism', Journal of Communication Inquiry 36.2 (2012): 149-169.****

Dunbar-Hester, Christina. 'Producing "Participation"? The Pleasures and Perils of Technical Engagement in Radio Activism', Public Culture, 26.1 (2014): 25-50.

Gerbaudo, Paulo. Tweets and the Streets: Social Media and Contemporary Activism (London: Pluto Press, 2012).

Giovanopoulos, Christos and Dimitris Dalakoglou. 'From Ruptures to Eruption: Genealogy of the December 2008 Revolt in Greece', in Antonis Vradis and Dimitris Dalakoglou (eds). Revolt and Crisis in Greece: Between a Present Yet to Pass and a Future Still to Come (Oakland, Baltimore, Edinburgh, London \& Athens: AK Press and Occupied London, 2011), 91-114.

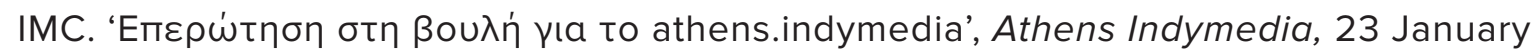
(2009), https://athens.indymedia.org/post/976610/

Inflammatory. 'A Map of Greece's Media Corrupt Relationships (part 1)', RadioBubble, 17 June (2013), http://international.radiobubble.gr/2013/06/a-map-of-greeces-media-corruptrelations.html

Kalyvas, Andreas. 'An Anomaly? Some Reflections on the Greek December 2008', Constellations 17.2 (2010): 351-365.

Karamichas, John. 'The December 2008 Riots in Greece', Social Movement Studies 8. 3 (2009): 289-293.

Kitis, E. Dimitris. 'The Anti-Authoritarian Chóros: A Space for Youth Socialization and Radicalization in Greece (1974-2010)', Journal for the Study of Radicalism 9.1 (2015): 1-36.

Kondilis, Elias, Giannakopoulos, Stathis, Gavana, Magda, Benos, Alexis, lerodiakonou, loanna and Waitzkin, Howard. 'Economic Crisis, Restrictive Policies, and the Population's Health and Health Care: The Greek Case', American Journal of Public Health 103.6 (2013): 973-979.

Kornetis, Kostis. 'No More Heroes? Rejection and Reverberation of the Past in the 2008 Events in Greece', Journal of Modern Greek Studies 28. 2 (2010): 173-197.

Koronaiou, Alexandra and Alexandros Sakellariou. 'Reflections on "Golden Dawn", Community Organizing and Nationalist Solidarity: Helping (only) Greeks', Community Development Journal 48.2 (2013): 332-338.

Kritidis, Gregor. 'The Rise and Crisis of the Anarchist and Libertarian Movement in Greece, 1973-2012', in Bart van der Steen, Ask Katzeff, and Leendert van Hoogenhuijze (eds).* The 
City Is Ours: Squatting and Autonomous Movements in Europe from the 1970s to the Present* (Oakland, CA: PM Press, 2014), 63-94.

Lowen, Mark. 'Greeks Strike Over Media Closure', BBC News, 13 June**(2013), http://www. bbc.co.uk/news/world-europe-22883236

Loza, Susana. 'Hashtag Feminism, \#SolidaritylsForWhiteWomen, and the Other \#FemFuture', Ada: A Journal of Gender, New Media, and Technology 5 (2014): http://adanewmedia. org/2014/07/issue5-loza/

Makrygianni, Vaso and Haris Tsavdaroglou. 'Urban Planning and Revolt: A Spatial Analysis of the December 2008 Uprising in Athens', in Antonis Vradis and Dimitris Dalakoglou (eds). Revolt and Crisis in Greece: Between a Present Yet to Pass and a Future Still to Come (Oakland, Baltimore, Edinburgh, London \& Athens: AK Press and Occupied London, 2011), $29-57$.

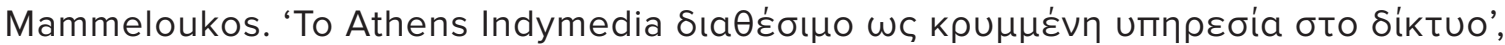
Athens Indymedia (squat.gr) 11 April**(2013), http://indymedia.squat.gr/2013/04/to-athens-

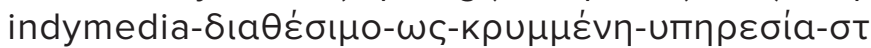

Margaronis, Maria. 'Fear and Loathing in Athens: The Rise of Golden Dawn and the Far Right', the Guardian newspaper, 26 October (2012), http://www.theguardian.com/ world/2012/oct/26/golden-dawn-greece-far-right

Marres, Noortje and Richard Rogers. 'Recipe for Tracing the Fate of Issues and their Publics on the Web', in Bruno Latour and Peter Weibel (eds). Making Things Public: Atmospheres of Democracy (Cambridge, MA: MIT Press, 2005), 922-935.

Meikle, Graham. Future Active: Media Activism and the Internet (New York, NY: Routledge, 2002).

Milioni, Dimitra L. 'Probing the Online Counterpublic Sphere: The Case of Indymedia Athens', Media, Culture \& Society 31.3 (2009): 409-431.

Milioni, Dimitra L. and Dionysis Panos. 'New Media and Radical Protest: Reflections from the "Greek 2008 Riots", International Journal of Media \& Cultural Politics 7.2 (2011): 233-240.

Mowbray, Mike. 'Blogging the Greek Riots: Between Aftermath and Ongoing Engagement', Resistance Studies Magazine (2010): 4-15.

Neumayer, Christina and Bjarki Valtysson. 'Tweet against Nazis? Twitter, Power, and Networked Publics in Anti-Fascist Protests', MedieKultur. Journal of Media and Communication Research 29.55 (2013): http://ojs.statsbiblioteket.dk/index.php/mediekultur/article/ view/7905

Occupied London Collective. 'Capitalism by Default', in Antonis Vradis and Dimitris Dalakoglou (eds). Revolt and Crisis in Greece: Between a Present Yet to Pass and a Future Still to Come (Oakland, Baltimore, Edinburgh, London \& Athens: AK Press and Occupied London, 2011), 328-331. 
Papacharissi, Zizi. Affective Publics: Sentiment, Technology, and Politics. (Oxford: Oxford University Press, 2015).

Psarras, Dmitris. The Rise of the Neo-Nazi party "Golden Dawn" in Greece: Neo-Nazi mobilisation in the Wake of Crisis. (Brussels/Athens: Rosa-Luxemburg-Stiftung, 2013).

Siapera, Eugenia. 'Golden Dawn and the Social Media Wars', The Press Project (2014, May 6), http://www.thepressproject.net/article/61294/Golden-Dawn-and-the-Wars-on-Facebook

Sotiris, Panagiotis. 'Rebels with a Cause: The December 2008 Greek Youth Movement as the Condensation of Deeper Social and Political Contradictions', International Journal of Urban and Regional Research 34.1 (2010): 203-209.

Theocharis, Yannis, Lowe, Will, van Deth, Jan W. and García-Albacete, Gema. 'Using Twitter to Mobilize Protest Action: Online Mobilization Patterns and Action Repertoires in the Occupy Wall Street, Indignados, and Aganaktismenoi Movements', Information, Communication \& Society 18.2 (2014): 202-220.

Tsing, Anna Lowenhaupt. Friction: An Ethnography of Global Connection (Princeton N.J.: Princeton University Press, 2005).

Tufekci, Zeynip. “"Not This One”: Social Movements, the Attention Economy, and Microcelebrity Networked Activism', American Behavioral Scientist 57.7 (2013): 848-870.

Tufekci, Zeynip and Christopher Wilson. 'Social Media and the Decision to Participate in Political Protest: Observations from Tahrir Square', Journal of Communication 62.2 (2012): 363-379.

Vatikiotis, Pantelis. 'Networking Activism: Implications for Greece', Estudos Em Comunicação 10 (2011): 163-179.

Vradis, Antonis and Dalakoglou, Dimitris (eds). Revolt and Crisis in Greece: Between a Present Yet to Pass and a Future Still to Come (Oakland, CA: AK Press and Occupied London, 2011).

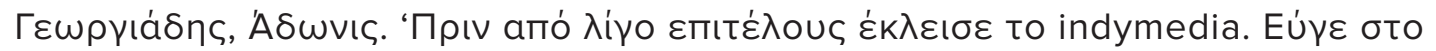

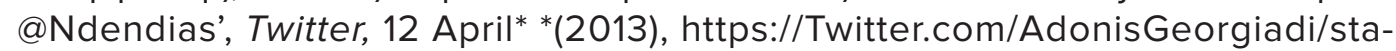
tus/322419853577318400

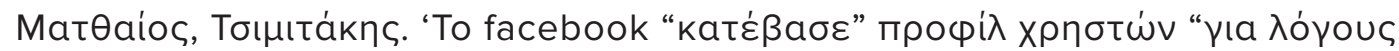

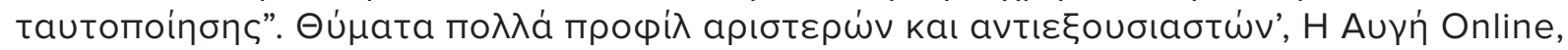
5 June (2013), https://www.avgi.gr/article/414765/to-facebook-katebase-profil-xriston-gialogous-tautopoiisis-thumata-polla-profil-aristeron-kai-antiexousiaston 
The LOCKSS System has the permission to collect, preserve and serve this open access

Archival Unit

This Isuue of the Fibreculture Journal by The Fibreculture Journal Incorporated is licensed under a Creative Commons Attribution 4.0 International License.

\section{OPEN HUMANITIES PRESS}

The Fibreculture Journal is published by The Fibreculture Journal Incorporated in partnership with Open Humanities Press. 


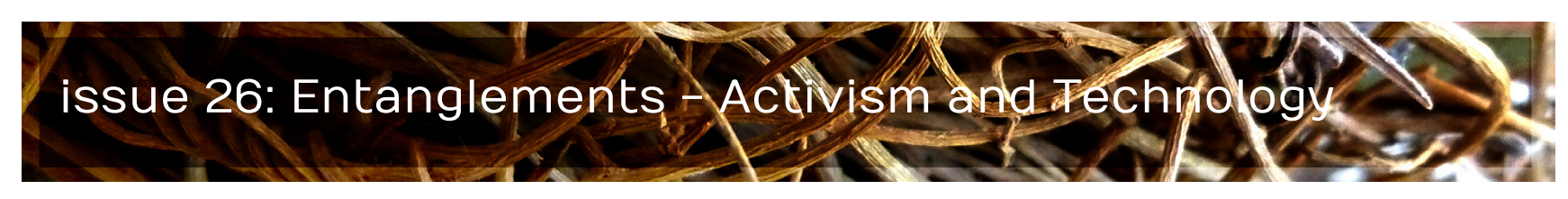

\title{
FCJ-194 From \#RaceFail to \#Ferguson: The Digital Intimacies of Race-Activist Hashtag Publics
}

Nathan Rambukanna

Wilfrid Laurier University

\begin{abstract}
:
This paper explores the rough, emergent and partial public culture of race-activist hashtags through the discourses of \#RaceFail, a critical race quarrel that started in the sci-fi and fantasy blogosphere, and expanded from there into a broader, sustained discussion over social media; and \#Ferguson, a recent race-activist hashtag raising issues around censorship, filtering and 'gated discourse'. It ends with a discussion of how the frictions between the neoliberal desire to reduce hashtag publics to product publicity, and the activist desire to use hashtags to further public sphere awareness of political issues, is exemplified in the controversy over Facebook's 'algorithmic filtering' of \#Ferguson, and how, nevertheless, critical race hashtags are tapping into a developing tradition of vocal social media-supported dissent.
\end{abstract}

doi: $10.15307 /$ fcj.26.194.2015 
Much has been written about the news potential of blogs and microblogs (see for example Siles, 2011; Papacharissi and Oliveira, 2012; Bastos et al., 2013), as well as about the political potential of online space in general (e.g., Bohman, 2004; DeLuca and Peeples, 2002; Downey and Fenton, 2003). In fact, since the dawn of the Internet age, discussion of the democratic potential of Internet-mediated space has been one of the major top level conversations. Yet a lot of that discussion gets mired in an orthodox Habermasian take on what we should consider a democratic public sphere-that is, one where rational critical discourse on matters of societal importance (such as, most critically, the actions of the State) can take place; populated by citizens stepping out of their private roles as interested individuals and into a public space where they become participants in disinterested discussion and debate (Habermas, 1962).

While one can argue the merits of Habermas's public sphere, what of the other kinds of discussion and debate that are facilitated by networked technology? Taking its cue from critical public sphere theorists such as Nancy Fraser (1992) and Michael Warner (2002), this paper explores those other publics: more-or-less subaltern, more-or-less rational, more-or-less critical, and almost certainly partial, affective, interested and loud. The paper is interested in angry publics. It is interested in fringe publics. It's interested in the kinds of publics that do politics in a way that is rough and emergent, flawed and messy, and ones in which new forms of collective power are being forged on the fly, and in the shadow of loftier mainstream spheres. These are the publics born of frictions, in Anna Tsing's sense, 'the awkward, unequal, unstable and creative qualities of interconnection across difference' (2005: 4).

Specifically, this paper unpacks the publics of the hashtag, that piece of multiply repurposed typography, that rebel punctuation mark moving to establish itself in new regimes of discourse and communication. Like other forms of active punctuation such as the '@', '\$' and '!', residents of QWERTY keyboards that, out of sheer convenience, were taken up by programmers and users alike to take on additional, performative meanings, the hashtag is now one of the most recognisable symbols of communication itself. From its humble Roman origins, where it was used to denote something was measured in pounds-a meaning that drifted towards additionally meaning something numbered (Roman, 2014) [1]-it was twice appropriated by early digital communication cultures. The first appropriation was in 1969 as the 'redial' button on early touch-tone phone keypads, where it was known as the 'octothorpe' (Roman, 2014), and the second was in 1988 as a symbol marking out IRC channels in the hay-day of early Internet chatroom enthusiasm (Zappavigna, 2011: 791). From its digital neonacy, it was then mobilised by Chris Messina, who, inspired by folksonomic endeavours such as del.icio.us, selected it due to its ubiquity on pre-smart phone keypads (pers. comm.) and proposed its use as a ground-up search symbol in tweets, a structure that was later worked into Twitter's programming language 
as an official function (Messina, 2007; Roman, 2014). Now, moving beyond Twitter alone and into other structures both on and offline, it has the potential to organise new structures of discussion, new 'ambient affiliations' (Zappavigna, 2011: 803), and new 'potential discourse communities' (2011: 801) where in-line metadata acts as both text and metatext simultaneously (2011: 801), drawing discourse together across technologies in a way that is worthy of more sustained study.

In the taxonomy of information types, hashtags are hybrids. They are both text and metatext, information and tag, pragmatic and metapragmatic speech. They are deictic, indexical-yet unlike other such signs that point elsewhere, hashtags point to themselves, to their own dual role in ongoing discourse. Some have argued that when it comes to the online organisation of information there's a tension of type between chronological and contextual modes (Benovitz, 2010: 124). Chronological organisation (for example, a thread) can sometimes fail to address the contextual embeddedness of a conversation; while contextual organisation (for example, a hyperlink) might be able to provide greater context (Benovitz, 2010: 124), yet itself risks losing the temporal aspect-how a topic has developed over time. The hashtag therefore functions in the space between the contextual and the chronological. It is a node of continued context across media, conversations and locales, and yet it emerges temporally, self-developing through time, pointing to itself as it points to the other texts it marks as within its ambit.

In this way hashtags push the boundaries of specific discourses. They expand the space of discourse along the lines that they simultaneously name and mark out. New hashtags are constantly being deployed into the world: some may not 'catch'; others might rise to prominence through repetition, through use, through uptake. For example, those organising or livetweeting a conference (for example, the 2014 Canadian Communication Association conference) might propose a number of hashtags-\#cca2014, \#cca14, \#cca, \#aca14-and some might become more popular than others, but all of them remain in play, becoming part of the ongoing flow. We could contrast this form of data organisation to processes such as agenda-setting, gatekeeping and creating news frames where those controlling messages set the shape of the discursive content from above, limiting the ways in which information flows may be organised and presented (Bastos et al., 2013: 264). While not discursive limiters or boundaries then, hashtags do have the ability to mark the discursive flows of an event, and are in fact events themselves (Bruns and Sauter, 2014; Rambukkana, 2014), straddling that dual role as text and metatext.

Drawing on thinkers such as Massumi (2011), Kwinter (2001) and Whitehead (1967), I conceive of events as moments when otherwise disparate experiences entwine to form something new. Threads of as-yet-unrelated material flow together in complex aggregates 
and assemblages that, once together, take on a novel character. They move from the complex to the singular, twining into threads of their own that go on to combine into further complexes, further assemblages. The gift that Foucault (1972) gave us for the figuring of discourse and discursive power was to draw our attention to discourse as event. As researchers, to understand the power and embeddedness of a specific discourse we need to trace it, to discover both its contextual relevance and its temporal arc-to trace both its affects and its points of origin, points of change. Where event theory can add to our understanding of the discursive is in showing how, in our current cross-linked and meta-textual environment, events of discourse are coming to recognise and mark themselves as such. This self-reflexive discourse occurs in multiple ways-not the least of which is the popular uptake of the term 'discourse' in its academic sense-but one worth highlighting due to its increasing societal prominence is through the political and discursive use of the hashtag.

Some previous studies have looked at the role of hashtags in relation to political discourse. Most of these are centred around analysing how the hashtag operates on Twitter during major-but temporally limited-political events. Hashtags such as \#Jan25, \#Tahrir, \#Egypt, \#Tunisia, and \#spill have been analysed, for example, with respect to their role in the Arab Uprisings (for example, Papacharissi and Oliveira, 2012), and a 2010 leadership upset in the Australian parliament (Bruns and Burgess, 2012). On a short timescale, events and studies such as these demonstrate how the hashtag can work as a uniting thread of discourse that allows those who use it to feed into an ongoing and evolving conversation-even, as Douai (2013) points out, acting as one of the seeds that can contribute to a critical mass forcing change. This paper, however, looks at the political hashtag on longer time scales, and for events that are ongoing. It looks at places where tensions simmer and build up over time, where frictions develop and ignite, and where those tracking these fraught politics through hashtags find new problematics and issues, drawing attention to them to wear away at normative and hegemonic phenomena. It also looks at how this kind of hashtag can spill beyond Twitter to other spaces of social networking-such as the recent introduction of the hashtag to Facebook and its more established use on Instagram-and even to other media spaces such as television and print, billboards, skywriting and graffiti (See Figure 1). But how exactly can we situate the hashtag with respect to political communication?

\section{Communication, Friction and Digital Intimacies}

As John Durham Peters (1999) reminds us, communication is not a process that naturally invites understanding, connection or societal harmony-despite a common conception that this is the case. This is perfectly illustrated in the tensions between the popular understanding of McLuhan's (1962) notion of the global village, and what the original 


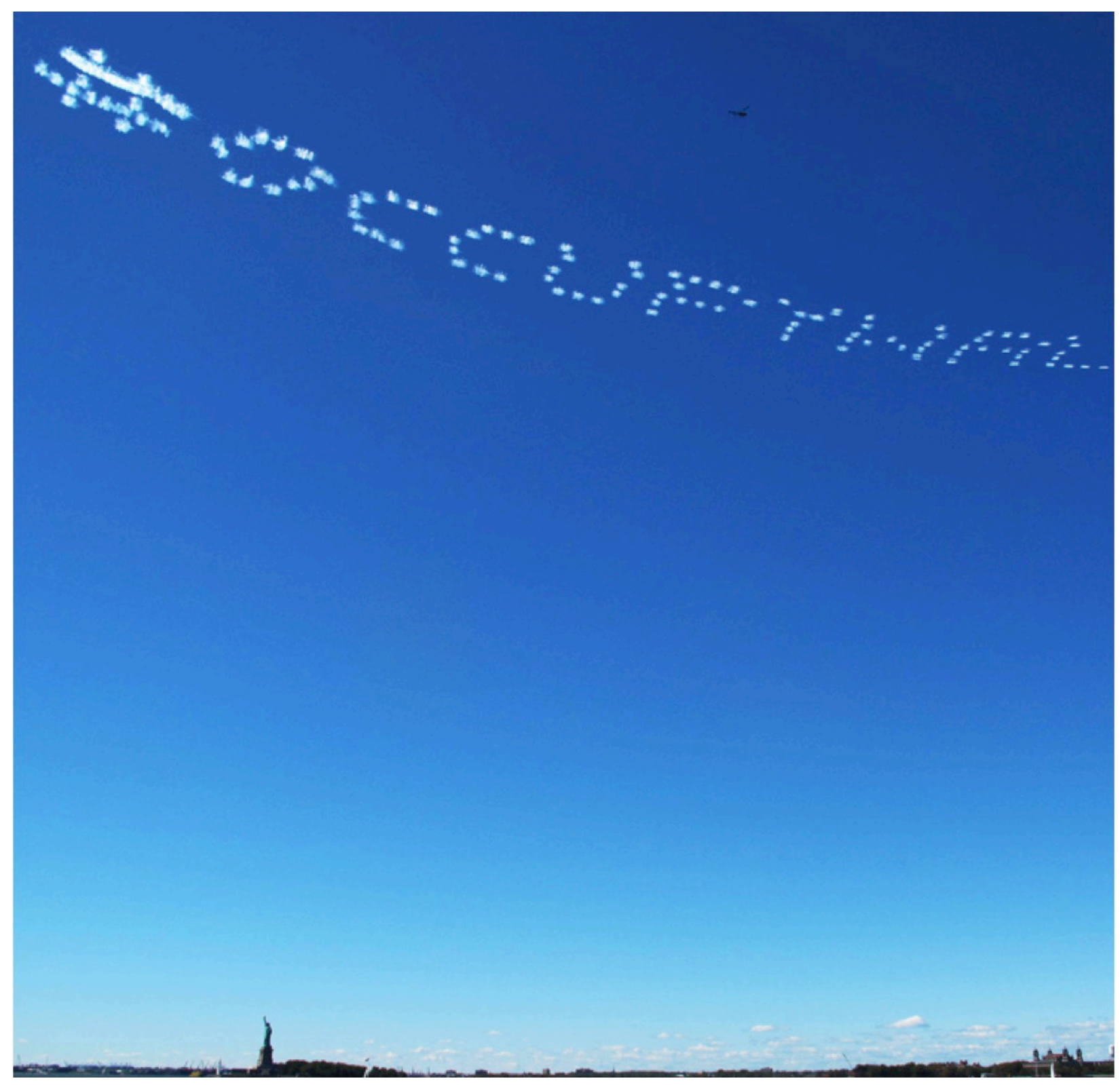

Figure 1: Occupy Wall Street Hashtag Skywriting (Harrington and Rojo, 2012)

text actually described. When I ask my students what they think 'global village' means, I usually get an answer that is akin to some cosmopolitan utopia, a borderless community where geographic distance no longer holds the same meaning and everyone is happy and connected. Then I get them to take apart the actual text. McLuhan deploys the 'tribal village' as a problematically racist metaphor, where the sound of war drums-heard by all in such a small space-gets everyone excited or angry. His argument that 'electronic interdependence recreates the world in the image of a global village' (1962: 31) has nothing to do with utopia. He envisioned a world where everyone is connected through these flows of information in ways that yes, draw us together, but he saw that drawing-together as fraught, complicated, even dangerous. We all hear the drums, no matter where we are; 
we all become embroiled in conflict, get in each other's business, in each other's space. Adding in digital media and the Internet-a natural extension to McLuhan's evocation of Pierre Teilhard de Chardin's noosphere, a vision of 'a technological brain for the world' (McLuhan, 1962: 32)-the global village might be better visualised not with utopic images of multiracial children holding hands, but with panoptic images of your boss confronting you with the Facebook wall pictures of your covert, alcohol-soaked, trip to Cuba, when you were 'officially' at home with the flu. And as with organisational communication, the same holds for international, and intercultural communication. Digital convergence increases the sphere of global connection, but that does not, in and of itself, heal a world community broken by misunderstanding. Rather, as Peters (1999) underlines, divergent understandings and interests are the natural baseline.

The political role of communication media cannot, therefore, be to 'fix' a broken system of public sphere communication, but rather to 'un-fix' staid communication patterns, to re-figure the public conversation about important issues and topics (such as inequality, racism, sexism and abuses of power) with a view to cracking open stable systems of meaning-making, and working-as Peters reminds us we need to do (1999: 30)-to build better communication across and between cultural and subcultural spaces. We need to work with and through the 'productive friction[s] of global encounters' (Tsing, 2005: $3)$, to tap the unpredictable and raw energy that is concomitant with our increasing interconnection.

And an increasingly prominent venue of such connections is in the realm of 'digital intimacies'. Intimacies, in the critical sense, are the 'kinds of connection that impact on people, and on which they depend for living' (Berlant, 1998: 284; original emphasis). For Lauren Berlant, the space of intimacy comprises close connections that matter and that subtend our lives and experiences, define multiple forms of human relationship, and act as crucial spaces of mediation between our selves and our worlds. The realm of the digital affords a multiplicity of new forms of intimate connection, especially now since, as Vincent Mosco discusses, digital technologies have sunk 'into the woodwork' (2004: 21) become part of the texture of our everyday lives. Nancy Baym even goes so far as to call cyberspace a myth (2010: 150); from her perspective, there is no separate space of digital connection, not any more. The digital has simply become one of the regular conduits of connection for everyday life-digital intimacies are now, quite simply, part of the new shape of human intimacy.

And the intimacies of our lives affect us; they forge, sustain, renew, break or alter connections that matter in our lives. And this is doubly so for intimate linkages and disassociations that might be working at an un-conscious level, that tap their affective 
vigor from some of the more problematic elements of status quo relationships, such as from racist, sexist, classist and ableist 'common sense' understandings. This is why it is important to investigate not only intimacy, but also 'intimate privilege' (Rambukkana, 2015). [2] One of the challenges in thinking through digital intimacies, then, is to think about what forms of intimacy might be privileged in the digital realm, as well as how the intimate privileges of society, and their resistance, surface in aspects of digital culture.

Which brings us back to the hashtag, the technosocial event (Rambukkana, 2014) that taps one of the privileged aspects of digital culture, virality, and uses it to promote and extend everything from advertising campaigns to activist engagements. In a world of increasing and cacophonous complexity, hashtags are singular moments that coalesce into something new: threads of meaning that work to weave new abstractions into the world. These new elements can range from the ridiculous to the sublime, from the politically irrelevant to the politically potent. It is this deeper end of the meme pool that is worth investigating further: What are these broader political functions of the hashtag? And how might something like race-activist hashtags form a kind of 'sticky engagement' (Tsing, 2005: 6) with these issues through time, and over distance? We will explore these questions through a singular but ongoing discursive event, RaceFail, and its affinities with subsequent tags that call out and expose racism in multiple venues of life and culture, most notably \#Ferguson.

\section{The Great Cultural Appropriation Debate and Racefail of Aught Nine}

As a case study that demonstrates the power of the hashtag as political discourse we can consider the sustained conversation about RaceFail that started in the science fiction and fantasy (SFF) blogosphere. Methodologically, this exploration mobilises Rogers's suggestion to 'follow the methods of the medium' as a way to 'reorient the field of Internetrelated research' to consider the Internet as a source of data, method and technique (Rogers, 2013: 1). By taking the object of study, '\#RaceFail/RaceFail' (with and without its 'hashed' diacritic), and employing multi-modal searches for its incidence as its major data-gathering methodology, this paper uses what might be termed a snowball textual analysis sample to gather material across media linked through the same tag. Unlike many early studies of hashtags that use programs such as TwapperKeeper to track and record tweets (e.g., see Bruns, 2012: 1331), this study centres the life of this tag in ways that contain but exceed the scope of the Twitterverse. The following texts were gathered using a number of tools, including Topsy, a website that provides the ability to search across social media platforms, and offers useful advanced search tools that, for example, allow searching the timeline for the earliest posts; as well as Google, for its far reaching search 
tendrils and metrics; and HootSuite in combination with Google Spreadsheets and TAGS Explorer to track and visualise more recent incidents on Twitter. Together, and through the cross-linking and archive posts found in many of the major blog posts on this topic, we can piece together a picture of this discursive event as it first exploded and then developed into a touchstone-the \#RaceFail hashtag, and the word RaceFail itself-that continue to be appended to ongoing discussions, working to evoke the sprit of the earlier debate and simultaneously stitch in new material.

'The Great Cultural Appropriation Debate and Racefail of Aught Nine,' AKA 'Writing the Other/Great Cultural Appropriation Debate of DOOM '09,' AKA 'RaceFail '09' stretches back as a named discourse to January of 2009. It was (and to a certain extent, still is) a messy, involved, heated, emotive discussion. In other words, it's the complete opposite of what Habermas would call 'rational critical discourse' (1981) and would be seen through his lens as a misuse of public sphere resources. But for several subaltern counterpublics (such as the SFF blogosphere, the antiracist blogosphere, and fan publics of multiple stripes) RaceFail '09 was a seismic shift in subcultural norms, trends and discussion. It kicked off a more visible critical race mediascape in SFF writing and criticism, as well on the net in general.

This online quarrel started in reaction to a blog post by speculative fiction author Elizabeth Bear about the politics and process of 'writing the Other' in science fiction and fantasy. Her post, initially a reaction itself to an earlier post on a related topic, downplayed the fraught politics of representation and presented a facile list of pitfalls to avoid and guidelines to follow to access what she characterises as a 'simple, but not easy' process (Bear, 2009). Her dismissal of the more nuanced arguments about the power relations and history of representing People of Colour and Indigenous people, coupled with what some read as racist narrative elements in her own work (Avalon's Willow, 2009), sparked first negative comments, then response posts containing further negative criticism. Collectively, these discussed (often heatedly) issues such as racism, cultural appropriation, colonialism, Orientalism, exoticism and white privilege-both in relation to SFF texts themselves and the norms of the SFF publishing industry-after which things got ugly (Somerville, 2013). Those critiquing Bear were themselves violently critiqued, mostly by what seem to be white fans/authors/publishing executives. While it is impossible to tell exactly who said what and when and to whom due to the pseudonymy of the Internet, the main tension (the major 'Fail') is how writers, fans and critics of colour were attacked about having the right to critique at all, characterising them as lacking the required intelligence and decorum for 'proper' intellectual debate. After this the quarrel descendeds into further personal attacks, with the spouses of the critiqued white critics, several of whom were also members of the publishing industry, jumping into the fray calling the of Colour-critics and allies 'trolls,' or 'orcs' and threatening to 'blacklist' them from industry publishing and fan culture, as 
well as outing one prominent critic by exposing her LiveJournal pseudonym, collectively collapsing the textual world of critique with that of interpersonal and professional politics (Somerville, 2013).

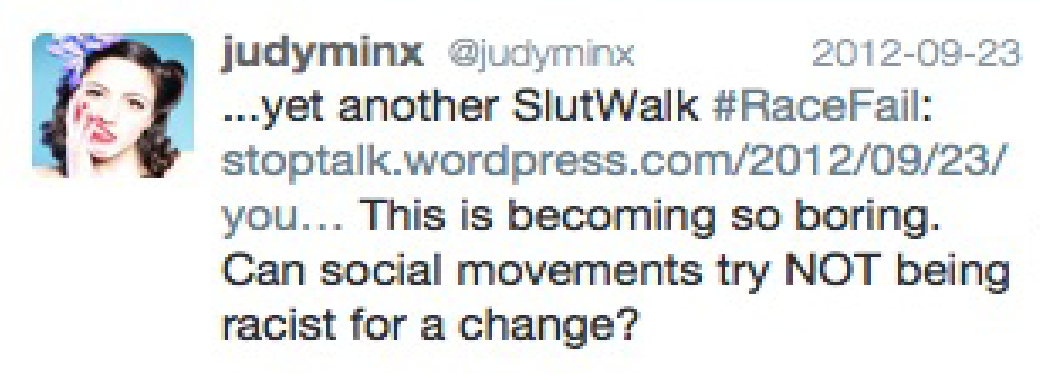

Figure 2: \#RaceFail Tweet about issues of Racism Surrounding the Toronto SlutWalk 2012

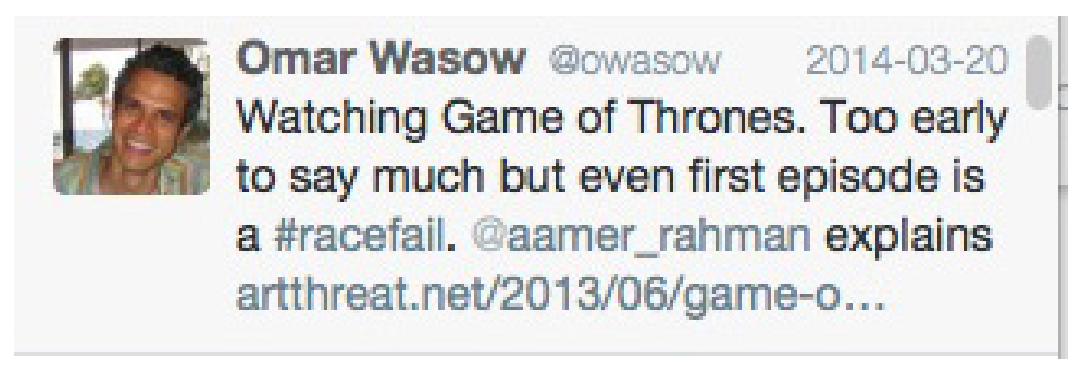

Figure 3: \#RaceFail Tweet Linking to Article about Racism in Game of Thrones

Collected around these loose main structuring events in the blogosphere is a nimbus of related discourse. The Topsy timeline indicates that while there were discussions of race in science-fiction and fantasy before this epoch-making epic web brawl, the emotions and issues thrown up in this scuffle were truly an 'incitement to discourse' in Foucault's (1976: 17) sense, with the keyword and then hashtag RaceFail-not present in web archives before this point-crystallising the affect of these debates into a diachronic anchor point that allows the thread to be pulled together across space and time. It is interesting, in particular, how this tagging had the ability to pull even-earlier material into its ambit. For example, this paper's snowball strategy retrieved many texts that that don't actually contain the term 'RaceFail', such as Bear's original post; however, by subsequent diachronic linking and archiving, and synchronic blog posts and comment threads, the hashtag collects and self-organises the material most relevant to it, linking back to incorporate even material that came before it, and helped it become. 
It is this linking function that is perhaps the most interesting. Now you can find the \#RaceFail hashtag across media platforms, and branching out to new subject matter. A review of Topsy findings collects material from Twitter, personal blogs, newsblogs, mainstream news sites, aggregators such as Tumblr and Reddit, and some wikis. At a second level of analysis, these posts and tweets, especially the later ones, utilise \#RaceFail to signify a discussion of racism in other spheres. It is in this sense that race-activist tagging, in addition to branching off to incorporate multiple media, starts to develop beyond the SFF public sphere to address other small publics as well as the mainstream public sphere as a whole. For example, there is discussion of mainstream movies, television and news, both within the realm of sci-fi and fantasy and beyond, with topics such as: the whitening of casting in The Last Airbender (2010) and The Hunger Games (2012); the sketchy race politics of episodes of BBC's Sherlock (2010); the cultural appropriation of various popular Halloween costume tropes; racist comments of/on mainstream politicians, actors or sports figures; even how race crosses with other issues, such as the politics of the SlutWalk (see Figure 2). Recent clusters show it being used, for example, to discuss the racist slave liberation narrative on HBO's Game of Thrones (2011) (see Figure 3), an unfortunate yellowface incident on CBS' How I met Your Mother (2005)that was also trending as a topic through the hashtag \#HowlMetYourRacism (Digital Spy, 2014)-and the whitewashing of Aronsfky's Biblical epic Noah (2014)**(Smietana, 2014; Sergio, 2014). Sometimes the hashtag is in the text itself, sometimes in the comments it incites, but every time the tag is used-and Google Trends show over 108,000 individual hits from a peak interest in mid-to-late 2009 through to mid-2014-a piece is added to the discussion.

However this doesn't mean to say that this discussion is unified, consistent or even entirely on topic. In fact, despite its persistence, the channel is a muddy one. For example, while the predominant use of \#RaceFail is as a race-activist hashtag, an important secondary usage rubbing up against it is about 'Fail' moments in relation to running culture, such as 'Aaaaaand it's raining \#racefail' (@SCRunnerGrl, 2015). Another, parallel, usage is a recent cluster that saw \#racefail being taken up to discuss a 'Fail' incident on the program The Amazing Race (2001), leading \#amazingrace to be the top hashtag used alongside \#racefail from September 2013 - March 2015, [3] an aberration that caused friction between the multiple publics it networked together, such as one user who tweeted '\#RaceFail usually means something very different in my circles \#AmazingRace' (@ haymakerhattie, 2014). Such usages, while they do 'muddy the waters' are not deliberate attempts to swamp the discussion (as discussed below), and are perhaps a low level frictive force we could think of as a form of noise. Another fraught element is how, while \#RaceFail does have a consistent presence across multiple platforms, it also often appears as Racefail, without the symbol, meaning that parts articulated to this hashtag public will only incidentally and inconsistently be collected by following the tag (for example, when an article is linked to, or a tweet retweeted). The fact that this hashtag public is not even always hashtagged as such-even on Twitter-is a further friction that underlines its provisional nature as a consistent public at all. 
Yet despite the inconsistencies and the misdirections, this discursive channel has still carved out a distinct and persistent political voice-but what does that critical voice do? How does this welling up of critical race discourse function with respect to those who deploy it, for fandom, for science fiction and fantasy, and for society in general? As $\mathrm{N}$. $\mathrm{K}$. Jemisin points out, it contributed to paradigm shift in these discursive spaces. On the rough anniversary of the explosion of RaceFail, critical race blogger and acclaimed fantasy writer N. K. Jemisin published a blog post titled 'Why I Think RaceFail Was The Bestest Thing Evar for SFF' (2010). In it she talks about how, contrary to some who think the 'Fail' part of RaceFail was that it happened at all, these issues needed to be discussed. But more: they needed to be argued. As Sara Ahmed notes, the policing of affect can have many negative effects, causing everything from psychological distress to bad politics. Subjects in precarious circumstances, such as immigrants, refugees, sessional workers, are often barred from expressing legitimate anger and are even subject to being forced to perform a kind of 'coercive happiness' in order to receive or retain access to common resources (2009). Fan cultures-and SFF fandom is particularly known for this-can perform some of that same policing, where anger with subcultural privilege and oppression around issues such as sexism and racism had been traditionally met by derision and exclusion.

Take for example \#GamerGate. \#GamerGate began as a targeted misogynist attack against game developer Zoe Quinn in 2014, involving public discussions of her sex life and accusations of collusion with game journalists to promote her game Depression Quest (Hathaway, 2014). This backlash against feminist games and game criticism became fused with the earlier backlash against Anita Sarkeesian's (2012) Kickstarter campaign to start a web series about sexism in video games, to which the response from some fans was the creation of sexist and misogynist memes, multiple death and rape threats, and even a flash video game the point of which was to beat Sarkeesian up to 'put her in her place' (Chee and Bergstrom, 2013). Together, this backlash, which frames itself rather as a symposium on ethics in games journalism (Hathaway, 2014), [4] has become an all-out-brawl, with much anger on either side. With this kind of staid culture, anger and intense discursive fighting are perhaps the only ways to bring about true change. As with \#GamerGate and the problem of sexism in video game culture, the quarrel-from the Latin, querel (or complaint)-that underliesay the question of race representation in SFF was always there waiting to happen; it was a disconnect, an aporia, a problematic issue that needed a prolonged and angry argument to air its grievances and map out their discursive extent. And as a discursive event, it bore fruit.

Jemisin notes that since RaceFail there has been an 'increased awareness [in] the SFF zeitgeist on race issues' (2010) that has led to many positive outcomes, including some publishers actively seeking out authors of colour (which, though risking tokenism also addresses a long-identified structural bias against authors of colour being considered 'real' SFF writers). It has also led to the publication of some excellent anthologies, and 
even speakers, panels or whole conferences devoted to issues of race in science-fiction and fantasy, such as Nalo Hopkinson being invited to give a keynote address at the International Association for the Fantastic in the Arts conference in 2010, and Jemisin herself being Guest of Honour at Continuum in 2013 (on which, more below). Jemisin's point is that these changes would not have occurred in the absence of the uppity spirit of \#RaceFail. Drawing on Lewin's theory of change, she writes about how:

stable organisations/systems inherently resist significant change, mostly due to inertia. They're frozen in place by the weight of their own history, the comfort of tradition, participants who have a vested interest in maintaining the status quo, and so on. So the only way to enact change in such a system is to destabilise it-unfreeze it. Then quickly push through changes before a new stable equilibrium is reached and the system freezes again (Jemisin, 2010).

From her perspective, RaceFail worked as a solidarity movement that 'turned up the heat' on an under-addressed issue (2010). It shook up a few stable systems (such as those of SFF fandom and publishing) creating new opportunities for ongoing discussion and debate that are having an ongoing material impact on the shape and nature of those publics. [5]

More recently, during her 2013 speech as Guest of Honour at speculative fiction and pop culture convention Continuum in Australia, Jemisin called for a discourse of reconciliation to keep alive this powerful discussion and, tapping the Australian model of reconciliation, redirect it into something positive, inclusive, even healing (2013). [6] After discussing her trepidation in coming to Australia (a country with a notorious history of racism) as an outspoken, young Black woman, she writes:

Australia may not be the safest place for someone who looks like me... but it's trying to become safer. And Australia may have classified the peoples of the Koori and other nations as 'fauna' until very recently, but Australia has also made tremendous strides lately in rectifying this error. I've listened in fascination to the Acknowledgements of Country made at nearly every public event I've attended since l've been here. I've marvelled that indigenous languages are offered as courses for study at some local universities. I am awed that you don't shove all of your indigenous history into a single museum, where it's easy for people not of that culture to avoid or ignore, because that's what happens in the US. So as horrified as I am by the nastier details of Australian history... I am also heartened, astonished, inspired, by the Australian present. You've still got a long way to go before Reconciliation is complete, but then again, you've started down that path. You're trying (Jemisin, 2013). 
She contrasts this with the situation in the United States where some are aggressively trying to repeal Black voting rights won in the Civil Rights era, where Stand Your Ground laws and similar in places such as Florida and Texas 'allow white people to shoot Black people with impunity,' as in the cases of Trayvon Martin and (we could add) Michael Brown, [7] and where even a fraught and limited reconciliation process would be an impossibly distant pipe dream (Jemisin, 2013). While the metaphor of Australia as a troubled political space on the road to rediscovery and renewal is perhaps a bit too simplistic, where she takes this argument with respect to SFF is a more fruitful, evoking the return of the repressed.

She calls for SFF to take up this spirit of reconciliation in order to unearth the hidden history of these genres. In a manner uncannily similar to how Amin Alhassan (2007) traces the histories of the theory of Marx, Foucault and Derrida to their post-colonial underpinnings (thus arguing that postcolonial theory should be taught as foundational to Communication and Cultural Studies), she Jemisin draws out the intimate privilege inherent in conceptualising SFF as white and male and western genres when there have been such stories and dreamings throughout human history and transcending geopolitical borders and boundaries. She writes:

We tread upon the mythic ground of religions and civilizations that far predate 'Western' nations and Christianity; we dream of traveling amid stars that were named by Arab astronomers, using the numbers they devised to help us find our way; we retell the colonisation stories that were life and death for the Irish and the English and the Inka and the Inuit; we find drama in the struggles of the marginalized and not-quite-assimilated of every society. Speculative fiction is at its core syncretic; this stuff doesn't come out of nowhere. And it certainly didn't spring solely from the imaginations of a bunch of beardy old middleclass middle-American guys in the 1950s (Jemisin, 2013).

Her call for reconciliation within these genres, at the levels of both fandom and publishing, is a call to harness the unease and anger into something generative, to use it to reclaim suppressed but central threads-to reweave the texture of SFF discourse into a stronger, more enduring fabric.

And among the elements she draws on in discussing how this could happen, she evokes the event of RaceFail in its ongoing digital persistence. She writes: 'If you did not follow RaceFail when it occurred or if you dismissed it as too much to handle, try. It's all still there; just Google it. Hundreds of people poured millions of words into articulating what's wrong 
with this genre, and how those wrongs can be made right. You owe it to yourself to read some of what they wrote' (Jemisin, 2013). Her words, both in the speech and in its archival on her blog and various other spaces, [8] further link this discussion to the tag of RaceFail, adding a further articulation to its ongoing meaning. [9]

As to how such discursive changes and resistances surface in broader publics, tracing some of the more recent uses of \#RaceFail brings us examples such as the apology for the yellowface incident on How I Met Your Mother, and the non-apology for the whitewashing of Aronofsky's Noah. [10] Akin to the Habermassian ideal of how the public sphere operates with respect to state power (turning over and deconstructing decisions, holding those making bad decisions accountable, putting civil society's desires and issues on the agenda) the publicity that accrues to RaceFail discussions on Twitter, Facebook news feeds, Tumblr, and even mainstream news channels is having some effect in the broader spheres of cultural production and of political discussion. For example, following the yellowface incident a flurry of tweets as well as posts in multiple other media (for examplee.g., Angry Asian Man, 2014; Racialicious, 2014; Ma, 2014) led showrunners Carter Bays and Craig Thomas to issue the following statement through a series of tweets:

Hey guys, sorry this took so long. @himymcraig and I want to say a few words about \#HowIMetYourRacism.

With Monday's episode, we set out to make a silly and unabashedly immature homage to Kung Fu movies, a genre we've always loved.

But along the way we offended people. We're deeply sorry, and we're grateful to everyone who spoke up to make us aware of it.

We try to make a show that's universal, that anyone can watch and enjoy. We fell short of that this week, and feel terrible about it (Digital Spy, 2014).

While the motivations behind this statement may be genuine or rhetorical, the effect of such an acknowledgement could bode well for more-mindful future cultural production-if only to avoid bad press. In contrast, we can also see how some creatives are resistant to change. In response to criticisms that Noah, a Biblical epic set in the Middle-East, had an exclusively white, British- or Australian-accented, cast, the co-screenwriter, Ari Handel, wrote: 
From the beginning, we were concerned about casting, the issue of race. What we realised is that this story is functioning at the level of myth, and as a mythical story, the race of the individuals doesn't matter. They're supposed to be stand-ins for all people. Either you end up with a Benetton ad or the crew of the Starship Enterprise (qtd. in Sergio, 2014).

He goes on to say:

You either try to put everything in there, which just calls attention to it, or you just say, 'Let's make that not a factor, because we're trying to deal with everyman.' Looking at this story through that kind of lens is the same as saying, 'Would the ark float and is it big enough to get all the species in there?' That's irrelevant to the questions because the questions are operating on a different plane than that; they're operating on the mythical plane' (qtd. in Sergio, 2014).

The recourse to the white-as-universal mythos is a symptom of the same reactionary response that sees attempts to redress structural privilege as 'politically correct' moves -the submerged logic being: change is difficult, and uncomfortable. But in the era of RaceFail, when such decisions are now being increasingly challenged and talked about, they no longer happen in a cultural vacuum where privileged representations of difference, race and world cultures simply become the representations. Like Jeff Rosenblum argues with respect to the impact of social media criticism on corporations in the film The Naked Brand (2013), in this era of high networked visibility through new media, public scrutiny can act as powerful motivator of corporate action-a way maybe to harness some of the pervasive energy of neoliberalism and wrest it towards (perhaps reluctant) social justice and social change. And, as with corporations, for the creatives working to fashion fictions these public reflections on representation, and the frictions they mobilise, will continue to have an impact on the stories they are putting out into the world. This matters because the stories we tell ourselves, even the science fictional and phantasmatic ones set in other worlds, are still populated with images and tropes of race and difference from the world we actually live in.

RaceFail acts as a persistent marker for these ongoing public sphere discussions. While the \#Fail hashtag predated RaceFail and was considered by some a persistent "microgenre" [used for] complaining about something, often technological' (Zappavigna, 2011: 803), \#RaceFail couples this persistent genre with a persistent politics, one that allows those ongoing discussions to weave their complexity into a singular-and powerful-discursive event. This kind of 'sticky engagement' (Tsing, 2006: 6) can even spill over into other cognate spheres of discourse. We now turn to a brief consideration of one such parallel discussion, that around \#Ferguson. 


\section{Ferguson and the Persistence of Race-Activist Hashtag Publics}

When police officer Darren Wilson shot and killed unarmed teenager Michael Brown on 9 August, 2014 in the small town of Ferguson, Missouri, the response of the predominantly African-American St. Louis suburb was one of disbelief, anger and protest (Kroh, 2014). While the story as a whole has multiple facets, extended over months and involving protests, a disproportionate police response and further sympathy and synergistic protests catalysed around subsequent events, such as the death of another Black teen Vonderrit Myers Jr. in October (Covert, 2014), the contours are all too familiar. The anger that emerges from these situations is a visceral and vital one, forged in long histories of injustice, resistance and exhaustion. Such is the raw amplitude of this frustration and anger that it imbues the social media discussions that reflect it with these qualities, as the affect of these struggles pours into this new medium of public discussion. Just as RaceFail exploded into the relatively new social spaces of the blogosphere, the shooting in Ferguson and its continuing aftermath have found a powerful articulation through social media discussions and in particular through the mobilisation of \#Ferguson.

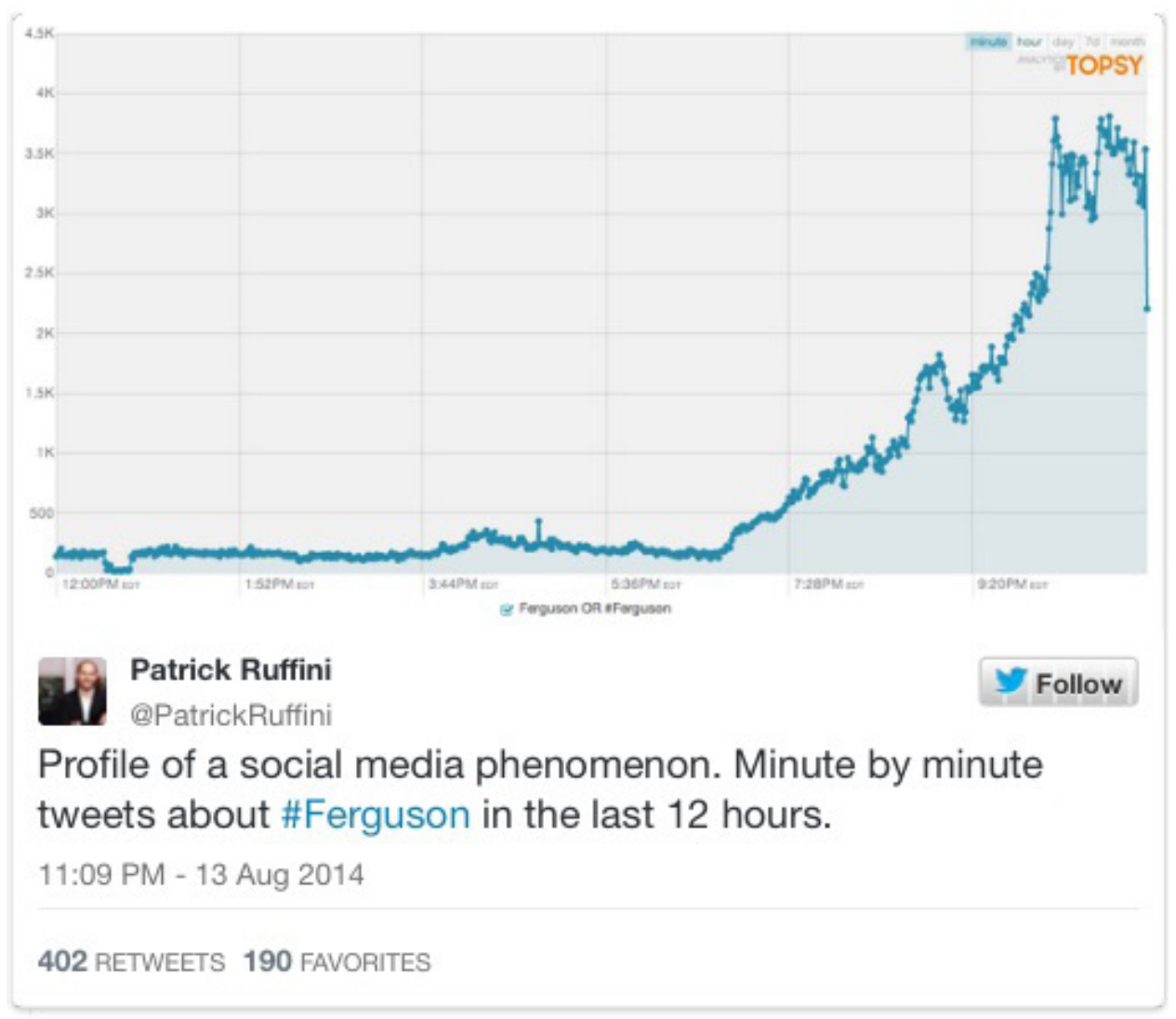

Figure 4: Patrick Ruffini's Topsy graph of \#Ferguson usages over first 9 hours. (Zerehi, 2014) 
Ferguson is the 9th most frequently-used hashtag in conjunction with \#RaceFail from September 2013 - March 2015, and arguably the most resonant one in mainstream news media, both US and international. Deployed soon after news broke about the events, the tag grew to over 4000 hits in only 9 hours (Zerehi, 2014; see Figure 4). Nor is it the only race-activist hashtag to emerge from these events. Others, such as \#FergusonOctober, \#IAmMichaelBrown and \#BlackLivesMatter, to mark the large October protest and highlight the repetitive occurrence of white police officers killing Black teens in the US; and \#ArrestDarrenWilson and \# JusticeForMikeBrown to call for justice in this case, were all prominently featured as part of these protests-and not just within tweets but on other social media platforms and even in analogue media such as protest signs (See Figure 5).

Appadurai discusses how primordia such as race have become globalised, writing that 'sentiments whose greatest force is their ability to ignite intimacy into a political sentiment and turn locality into a staging ground for identity, have become spread over vast and irregular spaces' (2010: 390). He was discussing evolving and spreading ethnoscapes as they spread through movements of people, images and techno-ideological flows, and the conjunction of race-issues and the hashtag can be seen as an apt example of the 'vast and irregular' new special formations he is theorising. Equal parts ethnoscape, ideoscape, mediascape and technoscape, [11] the space of the racialised hashtag public is one of powerful and mobile affectivity, drawing together and separating, calling out and memorialising, drawing a biopolitical problematic into a new sphere of human interaction.

That these issues have become globalised is undeniable. The Ferguson shooting and protests were featured on international and transnational media; for example, on the BBC (Petersen, 2014) and Al Jazeera (2014). One BBC news article even noted how Amnesty International was sending observers to monitor police actions and support the communities (a first for the United States), and that critics of the United States were piling on, with even Iran's Supreme Leader Ali Khamenei tweeting criticism of how the United States was treating its own citizens and using \#Ferguson in the tweet (Petersen, 2014). As Sauter and Bruns (2014) note, 'Thematic hashtags rarely exist in isolation from a wider discursive context: they emerge out of, are part of, and can shape events in the wider online and offline world.' While the social media articulation of \#Ferguson did not 'cause' this struggle to become global news, it is a significant part of its global articulation. Like how the portable videocamera became a crucial part of the event that was the Rodney King police beatings and their fraught aftermath, the political use of hashtags helped to ignite a global reflection on race, the policing of Black bodies and the militarisation of police, and became a crucial part of this new assemblage-despite and in direct competition to the neoliberal tendency of trying to transform the hashtag into an appendage of the advertising industry. 


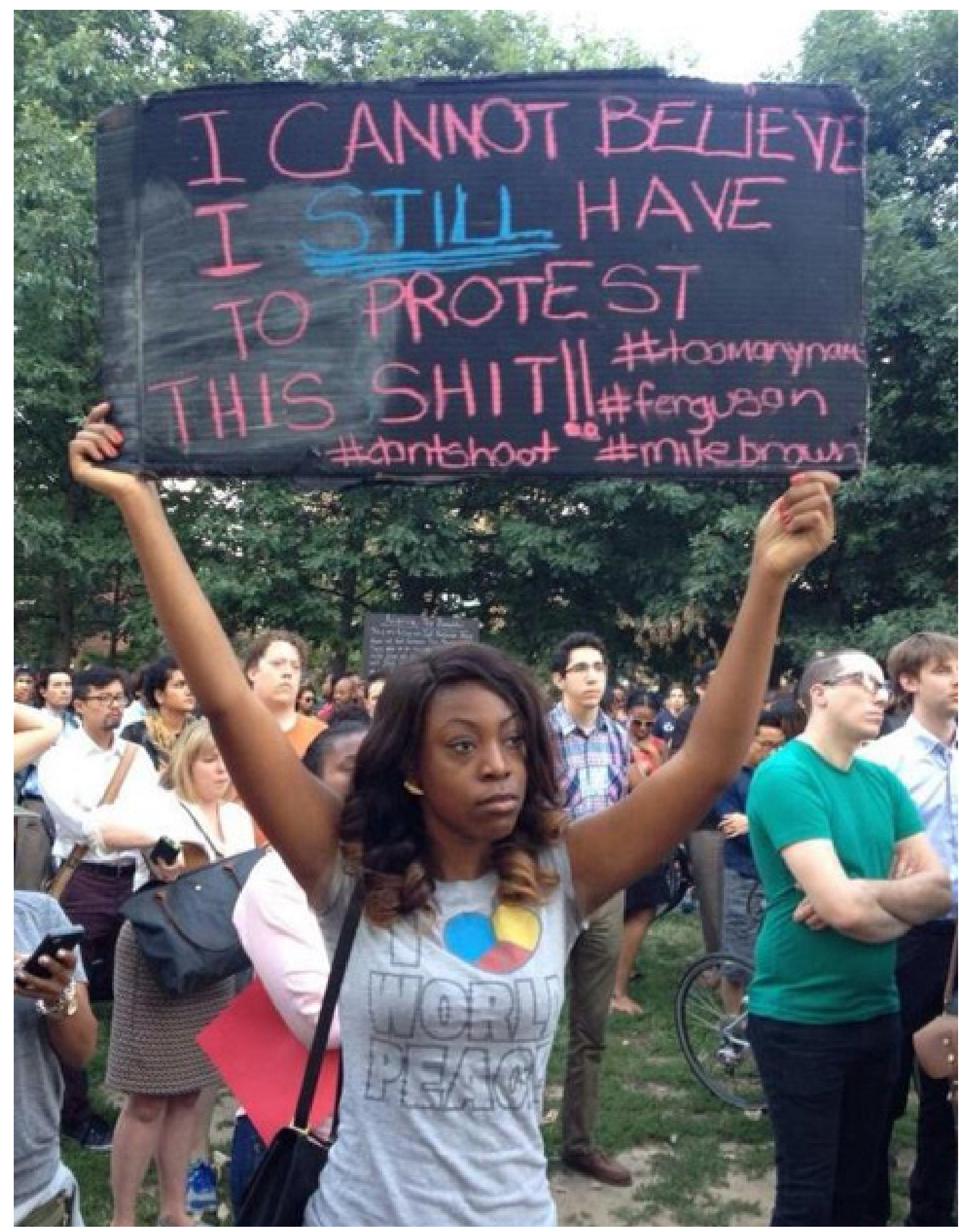

Figure 5: 'I Cannot Believe I STILL have to Protest this Shit' Sign, with Hashtags. (Starr, 2014).

The tension between this neoliberal desire to reduce hashtag publics to product publicity, and the activist desire to use hashtags to further public sphere awareness of political issues is codified in the controversy over the 'algorithmic filtering' (Tufekci, 2014) of \#Ferguson on Facebook. When many began to notice that \#Ferguson was trending on 
Twitter, but absent or near-absent on Facebook news feeds, critics started to reflect on the reason. One of the most prominent critics, Zeynep Tufekci, asks:

But I wonder: what if Ferguson had started to bubble, but there was no Twitter to catch on nationally? Would it ever make it through the algorithmic filtering on Facebook? Maybe, but with no transparency to the decisions, I cannot be sure. Would Ferguson be buried in algorithmic censorship? Would we even have a chance to see her? (Tufekci, 2014; See Figure 6).

The question of Facebook's algorithmic filtering and its effect (willed or not) spilled into mainstream media, with one CBC News article citing Anatoliy Gruzd, who noted that 'there's a concern that the algorithmic filtering of the content can hinder the ability of the protesters to build awareness for their particular campaign' through the use of hashtags on Twitter and Facebook (quoted in: Zerehi, 2014). The same article cites Fenwick McKelvey, who notes that this is not a new debate, with Twitter as well garnering criticism for their opaque and proprietary filtering practices. He notes that it might not be 'editorial bias that dictates what appears or doesn't appear on our timeline[s, but rather] algorithms intended to help us get a personalised experience on the web' (Zerehi, 2014). The collective point of these articles and voices is that such filtering practices affect and shape our personal

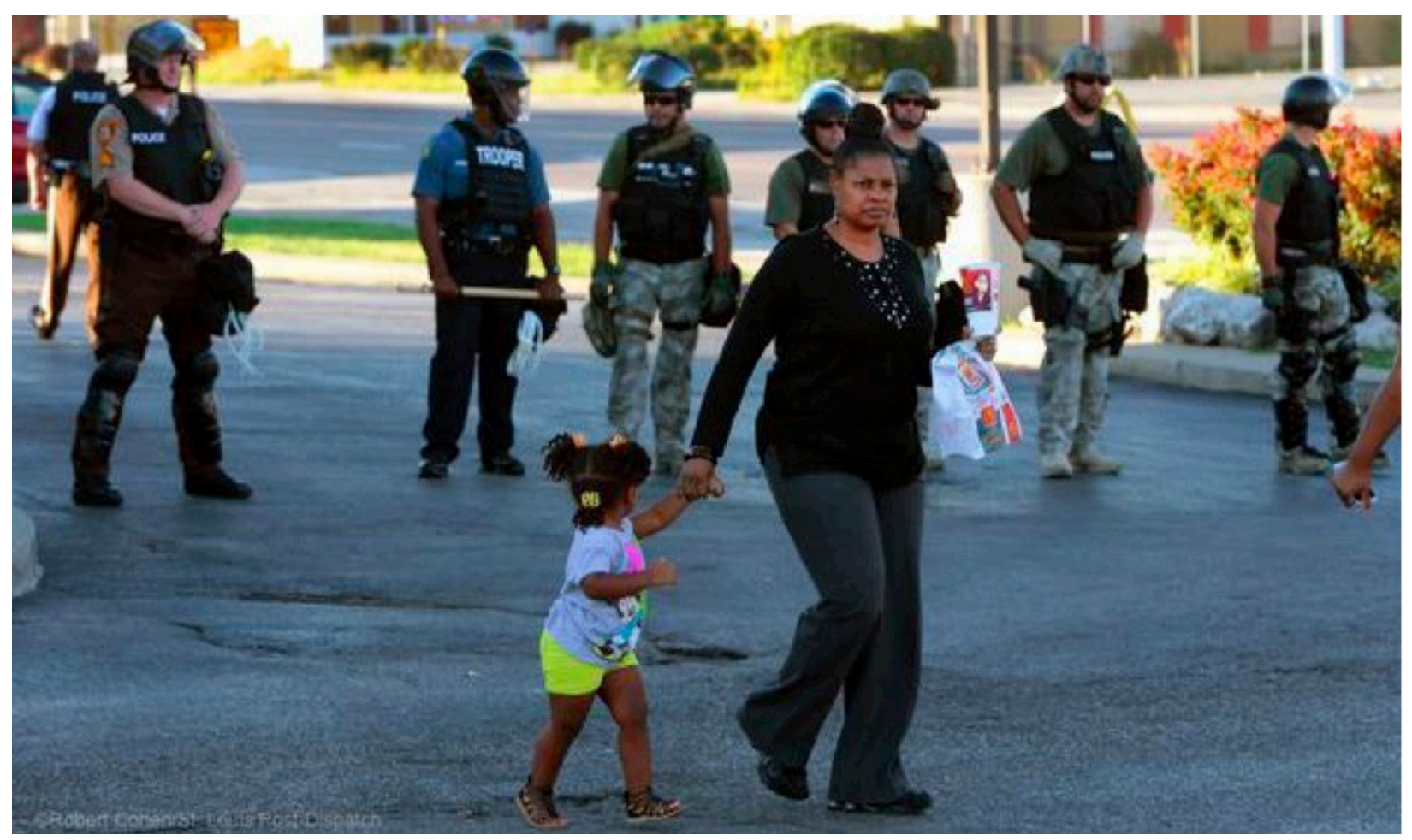

Figure 6: 'Would we even have a chance to see her?' (Tufecki, 2014) 
mediascapes-including, crucially, whether or not we will see controversial, fractious or political content-and as such our abilities to engage with such issues. While one can argue that it might not be incumbent on sites such as Facebook to curate our entire news consumption, the fact that software algorithms are being used to direct us toward certain content and away from others-or even to manipulate our moods (Meyer, 2014)-raises concern for issues as broadly situated as net neutrality, public sphere access, research ethics and digital media futures.

The social media mobilisation around \#Ferguson has led to an unprecedented response, including international news coverage and even transnational policy intervention. On 11 November, 2014, Michael Brown's parents spoke in front of the UN Committee Against Torture, where they:

call[ed] for global intervention in America's policing tactics. The couple asked a panel to recommend that 'racial profiling and racially-biased police harassment across the jurisdictions surrounding Ferguson' stops, and for the Department of Justice to 'conduct a nationwide investigation of systematic police brutality and harassment in black and brown communities, and youth in particular,' adding that the 'methodology and findings of this investigation must be made publicly available' (Townes, 2014).

This ongoing story would likely not have had the same sustained impact and international reverberation without its social media amplification-one that no doubt had a catalysing effect for those on the ground as well, acting as a global echo chamber for the sustained event of this loud and angry protest. At time of writing, people were waiting for the grand jury decision in the case of Darren Wilson and Missouri State Governor Jay Nixon was poised to call in the National Guard and also noted that over 1,000 police officers had completed over 5,000 hours of specialised training to prepare for this verdict, leading some commentators to note that given the preparation, the verdict seemed to be a

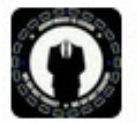

Anonymous Operations @AnonOpsSE.1h

\#Anonymous now in control of the main \#KKK twitter account @KuKluxKlanUSA \#OpKKK \#Ferguson \#lulz

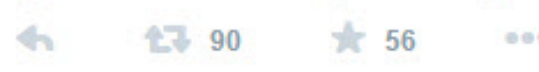

Figure 7: Anonymous's Tweet Announcing OperationKKK (Beaumont, 2014). 
forgone conclusion (Townes, 2014). Nixon went on to announce that these police measures were (ironically) so that peaceful protestors would not have their rights violated, adding that 'violence will not be tolerated' (citedqtd. in Rules, 2014). This statement, so out of step with Missouri's violent response to the first 90 day phase of this protest, and to the violent nature of policing of People of Colour in general, prompted journalist Julia Carrie Wong to post a series of images depicting victims of police violence with the repeated legend 'violence will not be tolerated' (Rules, 2014), one soon taken up as new viral hashtag often articulated in combination with \#Ferguson:

ViolenceWillNotBeTolerated @GovJayNixon Police fire teargas and rubber bullets into peaceful \#Ferguson protest (Rules, 2014).

And when the Klu Klux Klan (KKK) reared their unwelcome cowled heads as part of this discussion, announcing that they would use lethal force against Ferguson 'terrorists', Anonymous launched 'Operation KKK' to take over or block KKK social media, as well as un-hood and exposes its prominent members (Beaumont, 2004; See Figure 7). While still awaiting its final chapter, is it clear that this is a messy fight, and one in which social media in general, and race-activist hashtags in particular, are playing crucial parts.

\section{Conclusion: Beyond Mere Searching}

Searching and tagging are powerful technologies. They allow us to cut across contexts and gather material, texts, and resources. But beyond the individual process of searching and gathering like digital data, it is the self-consciousness and prominence of the hashtag that marks it as something worth noting-both in general and with respect to its potential impacts for activist politics and organising. While folksonomic tagging ventures and attempts to organise and archive conversations, debates, and fields of thought all break open exciting new spaces of discourse, none have achieved the mass mediated prominence of the hashtag. The frictions born from trying to wrest the hashtag away from the hollow articulations of public relations and advertising campaigns and maintain their potential for activist content will be ongoing. But one upshot of this prominence is, perhaps ironically, that at least in this one way neoliberalism and activism might be speaking the same language, though obviously with different intents. But might this mean a way out of the familiar cycle of just preaching to the choir in activist circles (Downing \& Rodriquez, 2005)? Do political hashtags, despite their inconsistent, somewhat-unfocussed, sometimesembattled aspects, have the world's ear? And do race-activist hashtags give us a new tool, not simply for a presumed-ineffectual 'armchair activism,' but for actual discursive intervention? 
One final question worth asking is: What is the space between \#RaceFail and \#Ferguson? One important thing to note of this gap is that the time between them isn't neutral, but rather one of development. The networked persistence of discussions of racism in life and culture (of which the stickiness of \#RaceFail is a useful metonym) has allowed critical race theory to expand from the bounds of too-insular activist and academic subcultures and publics into a known apparatus and structure of the public sphere as a whole. The space between these two tags is one of time and growth and development-similar, also, to the space between 9/11 and the Boston bombings or the recent shootings on Parliament Hill in Canada. When the tragedy of 9/11 happened, Western society snapped: racism, always already present and having major structural effects, flooded the public sphere and overwhelmed the critical voices and societal guilt-structures that kept it unevenly and incompletely in check. But in the subsequent events we had the apparatus of critical race discourse-forged on the fly from the frictions and fractures of that time combined with earlier writing and activism-to bulwark and embolden our critical voices; intersectional analyses to ask also what roles foreign policy, poverty and mental health issues might have had in triggering these horrible events; and the sobering memories of the dismal reality of the post-9/11 period to help us resist falling prey to the fear mongering of opportunistic and hawkish politicians. \#Ferguson and other new and potent race-activist hashtags (such as \#BlackLivesMatter, \#FreddieGray) are the legacy of earlier critical discussions and the earlier technosocial events that mobilise them, \#RaceFail being but one, and collectively form an activist critical objection-a quarrel-levelled at unjust abuses of power.

\section{Author Biography}

Nathan Rambukkana is an Assistant Professor in Communication Studies at Wilfrid Laurier University, in Waterloo Canada. His work centres the study of discourse, politics and identities, and his research addresses topics such as hashtag publics, digital intimacies, intimate privilege, and non/monogamy in the public sphere. He is the editor of the collection Hashtag Publics: The Power and Politics of Networked Discursive Assemblages, under contract with Peter Lang Press.

\section{Notes}

[1] The name and shape of the sign was likewise determined. It is known as the 'pound' or 'number' sign due to its Roman provenance, where it derived from the term Libra Pondo (pound in weight), which was abbreviated 'Ib', and evolved through usage and convention 
to ' $\square$ ', and finally '\#' (Roman, 2014). In the UK it is known as the 'hash sign' (a corruption of 'hatch' from its cross-hatched shape) since there pounds have their own distinct symbol (New Oxford American Dictionary, 2014). It is also sometimes known as the 'octotherp', 'octothorp' or 'octothorpe' and by other names in further contexts, such as the 'sharp' in musical notation-although typographically, this is a similar, though not identical, symbol: '\#'(Roman, 2014).

[2] 'Intimate privilege' is a concept I developed in my dissertation that examined discourses surrounding non-monogamy in the public sphere. It discusses how the social and cultural privileges that accrue to intimacies are emergent, how they ride in on a nexus of intersecting and interlocking privileges and oppressive elements to develop in singular ways. Intimate privilege, the amount of privilege one is able to mobilise in relation to one's intimate connections, is a factor of not just any one element alone, but the singular and situational combination of all of one's intimate connections.

[3] January 2013 was when I started to track this tag in real time. For a larger project I am currently seeking funding to get access to this tag through the now-proprietary Twitter API from its emergence in 2009.

[4] Both GamerGate and RaceFail show evidence of 'sea-lioning' a discursive technique where one side of a debate frames itself as more civil and reasonable than the other through persistent demands that the discussion be reframed along their lines and demands for evidence-and indeed this term was coined with respect to GamerGate (Don and Mercer, 2015). As a technique of discursive co-optation, it is similar to other techniques such as hashtag flooding, undue repetition of a tag to garner attention, and hashtag swamping, flooding a tag channel with unrelated or opposed information to blunt an activist message. This latter term was coined by \#Occupy organiser Susan Basko in 2011 to describe how those opposed to \#Occupy's message tried to render the channel informationally useless or 'hard to clean'-for example, through forcing organisers to waste time deleting posts and banning users (pers. comm., 2014).

[5] In a similar way, the feminist response to \#GamerGate and its precursors could be associated with a promising trend of both discussion of these issues, such as Anita Sarkeesian giving keynote addresses at the 2013 Feminists in Games conference in Vancouver (Yakashiro, 2013), and some changing representational trends, such as Blizzard announcing a new very muscular and not scantily-clad female Overwatch character named Zarya that was specifically designed to address these criticisms (Parfitt, 2015). 
[6] While we can be critical of discourses of reconciliation, especially with respect to how they can be coopted by settler states and mainstream media as ways to paper over and elide legitimate calls for anti-colonial resistance and redistributive justice (Wakeham, 2012), when substantial and not simply apologia for ongoing injustice, they can have 'radically transformative potential' (Wakeham, 2012: 1).

[7] Jemisin only mentions Trayvon Martin here, as the Michael Brown shooting in Ferguson, Missouri had not happened yet. I discuss this connection further with respect to the hashtag public that formed around \#Ferguson below, though it is worth nothing how this matter appearing in her speech foreshadows the persistence and unity of these publics.

[8] In addition to archiving the speech on her blog, the talk was discussed prominently elsewhere, including on Twitter, on various blogs (e.g., DeLong, 2013; Nicoll, 2013) and in the online periodical Dark Matter Zine ('Fall-out', 2013).

[9] Jemisin's further articulation and entreaty is one that has caused ripples. This move was met with some hostility, and such powerfully racist vitriol from science fiction writer Theodor Beale-he literally called her an uncivilised savage and hinted at her genetic inferiority (meadows, 2013)-that it is clear that the road to reconciliation in SFF might be long indeed.

[10] In its final season, an episode of the popular sitcom How I Met Your Mother was themed as a Samurai-style quest where a character seeks special powers from 'wise masters', all of whom were played by white actors portraying 'yellowface'-style characters, that is, charactersones based on East-Asian stereotypes and caricatures of East-Asian features, voices, etc. I unpack the context of the whitewashing of Aronofsky's Noah below.

[11] These are Apparudai's terms for the spaces and flows of ethnicity, ideology, media and technology respectively (2010: 384). 


\section{References}

@ haymakerhattie '\#RaceFail usually means something very different in my circles \#AmazingRace', Twitter, 27 April (2014), https://Twitter.com/HaymakerHattie/status/460579134868316161

@SCRunnerGrl. 'Aaaaaand it's raining \#racefail', Twitter, 22 February (2015), https://Twitter. com/SCRunnerGrl/status/569467101540421633

Ahmed, Sarah. 'Happiness, Race and Empire', Talk for International Day for the Elimination of Racial Discrimination, York University, Toronto, ON, 19 March (2009).

Al Jazeera. 'US opens Ferguson Civil Rights Inquiry', Al Jazeera, 5 September (2014), http://www.aljazeera.com/news/americas/2014/09/us-opens-ferguson-civil-rights-inquiry-20149422453226832.html

Alhassan, Amin. 'The Canonic Economy of Communication and Culture: The Centrality of the Postcolonial Margins', Canadian Journal of Communication 32 (2007): 103-118.

The Amazing Race (CBS, 2001).

Angry Asian Man. 'What's Up with the Yellowface on How I Met Your Mother?' Angry Asian Man [blog] 14 January (2014), http://blog.angryasianman.com/2014/01/whats-up-with-yellowface-on-how-i-met.html

Appadurai, Arjun. 'Disjuncture and Difference in the Global Cultural Economy', in Daya Kishan Thussu (ed.). International Communication: A Reader (London: Routledge, 2010), 383-392.

Avalon's Willow. 'Open Letter: To Elizabeth Bear', Seeking Avalon [blog] 13 January (2009), http://seeking-avalon.blogspot.ca/2009/01/open-letter-to-elizabeth-bear.html

Bastos, Marcos T., Rafael L. G. Raimundo and Rodrigo Travitzki. 'Gatekeeping Twitter: Message Diffusion in Political Hashtags', Media, Culture and Society 35.2 (2013): 260-270.

Baym, Nancy. Personal Connections in the Digital Age (Malden, MA: Polity, 2010).

Bear, Elizabeth. 'Whatever You're Doing, You're Probably Wrong', Throw Another Bear in the Canoe [blog] 12 January (2009), http://matociquala.livejournal.com/1544111.html

Beaumont, Valerie. 'Anonymous Hijacks KKK Twitter Account after Klan Declares Cyber War (Screenshots)', IfYouOnlyNews.com, 16 November (2014), http://www.ifyouonlynews.com/ racism/anonymous-hijacks-kkk-Twitter-account-after-klan-declares-cyber-war-screenshots/

Berlant, Lauren. 'Intimacy: A Special Issue.' Critical Inquiry 24.2 (1998): 281-288.

Benovitz, Miriam G. “'Because there aren't enough Spoons”: Creating Contextually-Organized Argument Through Reconstruction', National Communication Association Annual Conference (2010), Conference Proceedings, 124-130. 
Bohman, James. 'Expanding Dialogue: The Internet, the Public Sphere and Prospects for Transnational Democracy', The Sociological Review 54.1 (2004): 131-155.

Bruns, Axel. 'How Long is a Tweet? Mapping Dynamic Conversation Networks on Twitter using Gawk and Gephi', Information, Communication and Society 15.9 (2012): 1323-1351.

Bruns, Axel, and Jean Burgess. 'Researching News Discussion on Twitter: New Methodologies', Journalism Studies 13.5-6 (2012): 801-814.

Bruns, Axel and Theresa Sauter. '\#auspol: The Hashtag as Community, Event, and Material Object for Engaging with Australian Politics', in Nathan Rambukkana (ed.) Hashtag Publics: The Power and Politics of Discursive Networks, unpublished manuscript (2014).

Chee, Florence and Kelley Bergstrom. 'On Playing "Like a Girl": A Comparative Analysis of Quasi-Affirmative (Re)action', Paper presented at Canadian Communication Association, Victoria, BC (2013, 5-7 June).

Covert, Bryce. 'Largest Ferguson Demonstration Yet Ends With Sit-In', Think Progress, 13 October (2014), http://thinkprogress.org/justice/2014/10/13/3579229/ferguson-sit-in/

DeLong, Brad. 'N.K. Jemisin: Continuum GoH Speech', Brad DeLong's Grasping Reality... [blog] 13 August (2013), http://delong.typepad.com/sdj/2013/08/nk-jemisin-continuum-gohspeech.html?asset_id=6a00e551f08003883401901e1c172a970b

DeLuca, Kevin M., and Jennifer Peeples. 'From Public Sphere to Public Screen: Democracy, Activism, and the "Violence" of Seattle', Critical Studies in Media Communication 19.2 (2002): 125-151.

Digital Spy. 'How I Met Your Mother Creators Apologize for Yellowface Incident', Cosmopolitan, 15 January (2014), http://www.cosmopolitan.com/entertainment/tv/news/a18635/how-Imet-your-mother-apologizes-for-yellowface/

Don, and Mercer, Alex. 'Sea-lioning,' Know Your Meme (2015), http://knowyourmeme.com/ memes/sea-lioning/

Douai, Aziz. "Seeds of Change” in Tahrir Square and Beyond: People Power or Technological Convergence?', American Communication Journal 15.1 (2013): 24-33.

Downey, John, and Natalie Fenton. 'New Media, Counter Publicity and the Public Sphere', New Media \& Society 5.2 (2003): 185-202.

Downing, John, and Clemencia Rodriguez. 'Citizens' Media: Methods, Problems and Prospects', Keynote Address, Media Democracy Day. Concordia University, Montréal. October 18 (2005).

'Fall-out from N. K. Jemisin's Continuum 9 GpH Speech', Dark Matter Zine, 14 June (2013), http://www.darkmatterzine.com/fall-out-from-n-k-jemisins-continuum-9-goh-speech/

Foucault, Michel. The History of Sexuality Volume 1: An Introduction, trans. Robert Hurley (New York: Vintage, 1990; 1976). 
Foucault, Michel. 'Discourse on Language', trans. A. M. Sheridan Smith in The Archaeology of Knowledge and the Discourse on Language (New York: Pantheon. 1972), 215-237.

Fraser, Nancy. 'Rethinking the Public Sphere: A Contribution to the Critique of Actually Existing Democracy', in Craig Calhoun (ed.) Habermas and the Public Sphere (Cambridge: MIT Press, 1992), 109-142.

Game of Thrones (HBO, 2011).

Habermas, Jürgen. The Structural Transformation of the Public Sphere: An Inquiry into a Category of Bourgeois Society, Thomas Burger and Frederick Lawrence (trans.) (Cambridge, MA: MIT Press, 1989; 1962).

Habermas, Jürgen. The Theory of Communicative Action, Vol 1, Reason and the Rationalization of Society, trans. Thomas McCarthy (Boston, MA: Beacon Press, 1984; 1981).

Harrington, Steven and Jaime Rojo. 'Saber Tags Sky over New York to Defend Arts and Bash Romney', Brooklyn Street Art [blog] 23 September (2012), http://www.brooklynstreetart.com/theblog/2012/09/23/saber-tags-sky-over-new-york-and-bashes-romney/\#. VGoDAoeDh21

'Hash Sign', New Oxford American Dictionary (2014).

Hathaway, Jay. 'What is Gamergate, and Why? An Explainer for Non-Geeks,' Gawker, 10 October* *(2014), http://gawker.com/what-is-gamergate-and-why-an-explainer-for-nongeeks-1642909080

Hopkinson, Nalo. 'A Reluctant Ambassador from the Planet of Midnight', Journal of the Fantastic in the Arts 21.3 (2010): 339-350.

How I Met Your Mother *(CBS, 2005).

The Hunger Games (Lions Gate, 2012).

Jemisin, N. K. 'Why I Think RaceFail Was The Bestest Thing Evar for SFF', Epiphany 2.0 [blog] 18 January (2010), http://nkjemisin.com/2010/01/why-i-think-racefail-was-the-bestestthing-evar-for-sff/

Jemisin, N. K. 'Continuum GoH Speech', Epiphany 2.0 [blog] 8 June (2013), http://nkjemisin. com/2013/06/continuum-goh-speech/

Kroh, Kiley. 'Missouri Town Erupts In Protest After Police Shoot Unarmed Black Teenager', Think Progress, 10 August (2014), http://thinkprogress.org/justice/2014/08/10/3469602/ ferguson-police-michael-brown/

Kwinter, Sanford. 'The Complex and the Singular', Architectures of Time: Toward a Theory of the Event in Modernist Culture (Cambridge, MA: MIT Press, 2001), 3-31.

The Last Airbender (Paramount, 2010).

Ma, Kai. 'Dear, “How I Met Your Mother”: "Asian” Is Not a Costume' Time, 17 January (2014), http://time.com/1155/dear-how-i-met-your-mother-asian-is-not-a-costume/ 
Massumi, Brian. Semblance and Event: Activist Philosophy and the Occurrent Arts (Cambridge, MA: MIT Press, 2011).

McLuhan, Marshall. The Gutenberg Galaxy (Toronto: University of Toronto Press, 1962).

meadows, foz. 'Reconciliation: A Response To Theodore Beale', shattersnipe [blog] 14 June(2013), https://fozmeadows.wordpress.com/2013/06/14/reconciliation-a-response-totheodore-beale/

Messina, Chris. 'Groups for Twitter; or A Proposal for Twitter Tag Channels', FactoryCity [blog] 25 August (2007), http://factoryjoe.com/blog/2007/08/25/groups-for-Twitter-or-aproposal-for-Twitter-tag-channels/

Meyer, Robinson. 'Everything We Know About Facebook's Secret Mood Manipulation Experiment', The At/antic, 28 June (2014), http://www.theatlantic.com/technology/archive/2014/06/everything-we-know-about-facebooks-secret-mood-manipulation-experiment/373648/

Mosco, Vincent. The Digital Sublime: Myth, Power, and Cyberspace (Cambridge, MA: MIT Press, 2004).

Nicoll, James. 'N.K. Jemisin's Continuum GoH Speech', More Words, Deeper Hole [blog] 8 June (2013), http://james-nicoll.livejournal.com/4403512.html

Noah* (Paramount, 2014).

The Naked Brand (Questus, 2013).

Papacharrissi, Zizi and Oliveira, Maria de Fatima. 'Affective News and Networked Publics: The Rhythms of News Storytelling on \#Egypt', Journal of Communication 62 (2012): 266282.

Parfitt, Ben. 'Blizzard Hopes New Overwatch Character will Help Address Female Diversity Criticisms', MCV: The Market for Computer \& Video Games, 9 March (2015), http://www. mcvuk.com/news/read/blizzard-hopes-new-overwatch-character-will-help-address-femalediversity-criticisms/0146344

Peters, John Durham. Speaking Into the Air: A History of the Idea of Communication (Chicago, IL: University of Chicago Press, 1999).

Petersen, Kierran. 'Unrest in Ferguson Draws Attention from the US's Critics', BBC News, 20 August (2014), http://www.bbc.com/news/blogs-echochambers-28845076

Racialicious. Untitled Post. tumb/r, 15 January (2014), http://racialicious.tumblr.com/ post/73432116354/as-if-you-didnt-have-enough-of-a-reason-to-hate

Rambukkana, Nathan. Fraught Intimacies: Non/Monogamy in the Public Sphere (Vancouver, BC: UBC Press, 2015).

Rambukkana, Nathan. 'Introduction: Hashtags as Technosocial Event', in Nathan Rambukkana (ed.) Hashtag Publics: The Power and Politics of Discursive Networks, unpublished 
manuscript (2014).

Rogers, Richard. Digital Methods (Cambridge, MA: MIT Press, 2013).

Roman. 'Episode 145: Octothorpe', 99\% Invisible [online radio program] 16 December (2014), http://99percentinvisible.org/episode/octothorpe/

Rules, Cassandra. 'Missouri Governor Says "Violence Will Not Be Tolerated" In Ferguson, A Viral Hashtag is Born', The Free Thought Project, 12 November (2014), http://thefreethoughtproject.com/missouri-governor/\#ur3MJ5KdkqbaMTbL.99

Sergio. 'Co-Screenwriter of "Noah" Explains Why There Are No Black People Or POC In The Film', Shadow and Act [blog] 14 April (2014), http://blogs.indiewire.com/shadowandact/ co-screenwriter-of-noah-explains-why-there-are-no-black-people-or-poc-in-the-film

Sherlock (BBC, 2010).

Siles, Ignacio. 'The Rise of Blogging: Articulation as a Dynamic of Technological Stabilization', New Media \& Society 14.5 (2011): 781-797.

Smietana, Bob. 'Does "Noah" Have A Race Problem? Biblical Film Draws Criticism For Lack Of Diversity', Huffington Post, 4 August (2014), http://www.huffingtonpost.com/2014/04/08/ noah-race_n_5107490.html

Somerville, Ann. 'A Themed Summary of RaceFail '09', Fiction by Ann Somerville: Love, Romance, and the Occasional Sound Thrashing blog.. http://annsomerville.net/a-themedsummary-of-racefail-09-in-large-friendly-letters-for-those-who-think-race-discussions-arehard/

Starr, Terrell J. 'Woman Behind Powerful Mike Brown Protest Photo Defies "Respectability Politics"', AlterNet, 17 August (2014), http://www.alternet.org/woman-behind-powerful-mikebrown-protest-photo-defies-respectability-politics

Townes, Carimah. “'Ready For War”: 1,000 Police Officers Mobilized In Advance Of Grand Jury Ruling In Ferguson', ThinkProgress, 12 November (2014) http://thinkprogress.org/justice/2014/11/12/3591240/ferguson-grand-jury-ruling-prep/

Tsing, Anna Lowenhaupt. Friction: An Ethnography of Global Connection. (Princeton N.J.: Princeton University Press, 2005).

Tufekci, Zeynep. 'What Happens to \#Ferguson Affects Ferguson: Net Neutrality, Algorithmic Filtering and Ferguson', Medium.com, 14 August (2014), https://medium.com/message/ ferguson-is-also-a-net-neutrality-issue- $6 \mathrm{~d} 2 \mathrm{f3} \mathrm{db} 51 \mathrm{eb0}$

Wakeham, Pauline. 'Reconciling "Terror": Managing Indigenous Resistance in the Age of Apology', American Indian Quarterly 36.1 (2012): 1-3.

Warner, Michael. Publics and Counterpublics (New York: Zone Books, 2002).

Whitehead, Alfred North. Process and Reality, Corrected edition (New York: Free Press, 1967). 
Yakashiro, Emily. 'Feminists in Games Meet in Vancouver', Gender Focus (2013, 5 June).

Zappavigna, Michelle. 'Ambient Affiliation: A Linguistic Perspective on Twitter', New Media \& Society 13.5 (2011): 788-806.

Zerehi, Sima Sahar. 'Michael Brown's Shooting in Ferguson Lost on Social Media', CBC.ca, 19 August (2014), http://www.cbc.ca/news/technology/michael-brown-s-shooting-in-ferguson-lost-on-social-media-1.2740014

\section{OPEN HUMANITIES PRESS}

The Fibreculture Journal is published by The Fibreculture Journal Incorporated in partnership with Open Humanities Press. 


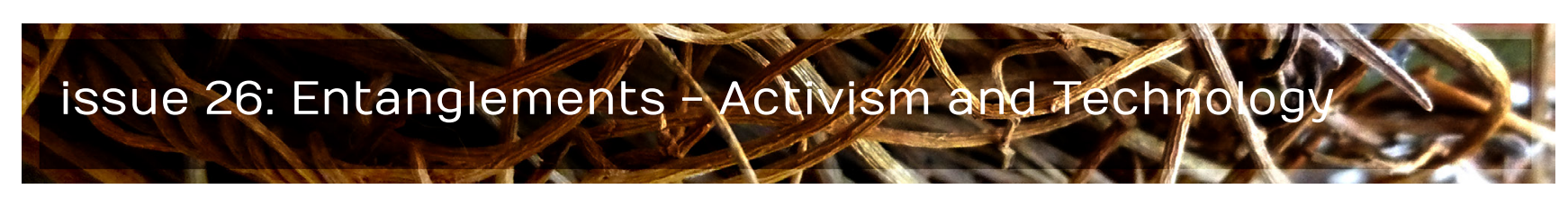

\title{
FCJ-195 Privacy, Responsibility, and Human Rights Activism
}

\section{Becky Kazansky}

Tactical Technology Collective

\begin{abstract}
:
In this article, we argue that many difficulties associated with the protection of digital privacy are rooted in the framing of privacy as a predominantly individual responsibility. We examine how models of privacy protection, such as Notice and Choice, contribute to the 'responsibilisation' of human rights activists who rely on the use of technologies for their work. We also consider how a group of human rights activists countered technology-mediated threats that this 'responsibilisation' causes by developing a collective approach to address their digital privacy and security needs. We conclude this article by discussing how technological tools used to maintain or counter the loss of privacy can be improved in order to support the privacy and digital security of human rights activists.
\end{abstract}

doi: $10.15307 /$ fcj.26.195.2015 


\section{Introduction}

Tactical Technology Collective (Tactical Tech) is an international Non-Governmental Organisation focused on supporting the effective use of information in advocacy. Tactical Tech has spent a decade listening to, documenting, and responding to activists' privacy and digital security needs and challenges across the world, often in contexts where the free flow of information is constrained. This vantage point has allowed Tactical Tech to observe the transnational spread of digital surveillance technologies, and their use against human rights activists (Hankey and O'Clunaigh, 2013; Notley and Hankey, 2013). Stories of monitoring and intrusion facilitated through digital surveillance technologies have been relayed to Tactical Tech in training events, through research and documentation field trips, at conferences and workshops, and via networks and activist media. These stories confirm the kinds of harms described and theorised in surveillance studies literature and in reports by civil society organisations documenting the psychological effects of mass surveillance, such as anxiety (Bigo, 2006), 'anticipatory conformity' (Braman 2006, 130), and 'self censorship' (Human Rights Watch, 2014; Pen America, 2013). These stories also confirm that human rights activists are often made key targets for surveillance because they challenge powerful interests, expose injustices and make rights claims in repressive environments, while they also provide evidence that surveillance can end in physical harm or arbitrary and unjust imprisonment (Citizen Lab, 2014).

Despite the substantial risks they face, human rights activists, like all 'users' of networked technologies, are tasked with the responsibility of managing their personal information in a way that supports privacy and security. We use the terms 'privacy' and 'security' throughout this paper in a complementary fashion. Privacy refers to the anonymity and confidentiality of information shared and stored through devices such as phones and laptops, and through digital platforms such as Facebook or Google. 'Digital security' refers to the concrete strategies or actions that respond to or resist a lack or loss of privacy; this involves the development and/or implementation of practices that can enhance anonymity or ensure confidentiality while also mitigating the consequences of data loss and intrusion. Overall, or 'holistic security'(Tactical Technology Collective, 2014) in human rights work is contingent upon the use of strategies for managing information which tie privacy to the physical protection and wellbeing of human rights activists.

One challenge human rights activists face in designing and implementing digital security strategies is that simply starting this process requires substantial technological knowledge. Like all users, protecting their privacy requires that they understand the properties and extent of 'data traces' left behind when using online consumer services and software; that they know the complex legal rights they have through commercial platforms' Terms of 
Service (TOS); that they be able to manage the technological options available to change default user settings; and that they are able to apply additional technological remedies to compensate for the lack of protection or control such platforms provide. The additional high level risks and threats posed by contemporary digital surveillance and intrusion in the context of human rights work also demands that concerned activists learn how to investigate potential data leaks from their devices and those of their colleagues, friends or personal networks. Yet this is a complicated endeavour: activists and human rights organisations often have limited technological support, while sophisticated surveillance attacks are so complex that only a tiny cadre of digital forensic experts are able to investigate with certainty whether a device has been technically compromised or not.

As part of our work supporting the digital privacy and security of human rights activists, we undertook field research during the period October 2013 to November 2014 to better understand how capacity building interventions such as digital security trainings figure into constructive responses to surveillance, privacy breaches, and overall security concerns in human rights activism. This research, part of our 'Security in Context' research project looked specifically at what factors cause concern over privacy and security to first arise, how digital security practices spread between individuals, groups, and networks, and how trainings aid these trajectories of awareness and learning. This work was founded on Tactical Tech's belief that issues around privacy and security must be engaged within a contextually appropriate way (Tactical Technology Collective and Front Line Defenders, 2015). To meet this aim, our capacity building efforts, such as the trainings that we conduct, start with a 'context analysis' of social, political and technical factors affecting human rights work at a particular place and time. This process serves to bridge knowledge of relevant threats between training facilitators and participants. Following this form of assessment, strategies are created and tools are chosen based on an emergent mutual understanding of the most salient concerns. This training approach directly informed the mixed method approach of our research, which utilised surveys, semi-structured interviews, workshopbased discussion and participatory activities with a total of 40 participants based in four different countries.

Reporting on some of the findings from this study, this article highlights the experiences of a network of women's and LGBTQI rights activists who set out to address their privacy and security concerns collectively in order to counteract digital surveillance, online harassment, threats of physical violence, and in some cases, physical violence. Because the use of Facebook has figured so prominently in their work, we focus on Facebook in this article as a platform for organising, as a technological interface with a certain set of properties or affordances which affect the kinds of control available to a user concerned with privacy, and as a company with policies that reflect a particular culture and business model. Our research finds that while Facebook was invaluable to the activists for organising protests 
and actions and later in creating strategies of self-defence, this platform also proved to be one of their greatest points of vulnerability. Already challenged by a large resource differential between themselves and their opponents, this imbalance of power was compounded by activist's forced reliance on an opaque, commercial platform offering little protection or control to its users. We call this a forced reliance because Facebook, as a networking platform, facilitates the maintenance of existing social ties and serves as a popular and dominant channel through which new relationships are created and sustained, and because there is no comparable replacement available. Baumer et al (2013) describe this forced reliance as a form of 'lagging resistance' wherein users express high levels of dissatisfaction with a tool but ultimately continue to use it for lack of viable alternatives. The phenomenon of lagging resistance demonstrates a systematic failure to provide users with adequate choices, protections, or controls over their privacy.

\section{Digital Privacy and Responsibility}

Conflicting views among software developers, activists, and policy makers reflect ambivalence over where primary responsibility for the protection of privacy should lie. Free Software luminary Richard Stallman starts his article titled 'How Much Surveillance Can Democracy Withstand?' (2014), by admonishing: 'First, don't be foolish. To have privacy, you must not throw it away.' Stallman's view reflects an expectation that users assume control and exhibit common sense in their actions using online tools. Stallman then outlines steps for users to take back control of their data, but goes on to admit that 'self-protection is essential, but even the most rigorous self-protection is insufficient to protect your privacy on or from systems that don't belong to you.' What kind of control users can realistically take in light of the complexity of risks, lack of meaningful alternative choices, and lack of accountability by companies, becomes the ongoing question for those creating privacy enhancing technologies or advocating strategies for greater privacy and security.

For activists working to defend human rights, it can be difficult to reconcile the need for and demands of self-protection with the feeling that the nature of their work should not require having to adopt a vigilant, self-defensive posture. One women's rights activist we interviewed, who, in her use of Facebook faces regular harassment and hacking attempts by a concerted collusion between state and state-affiliated actors, explained: "I want people who are doing things that are making me digitally insecure to not do those things. I feel a tension in having to assume that responsibility.' [1]

The process through which technology users are granted primary stewardship over 
their digital privacy has been characterised by scholars working on privacy enhancing technologies as a form of 'responsibilisation' (Gürses, 2014; De Wolf, Heyman and Pierson, 2014). They enter into a dependent relationship with opaque technologies, and are effectively left no choice but to deal with ensuing threats individually. The term responsibilisation originates from an interrogation of the privatisation under way in the 1980s in the United Kingdom and the United States, and the neoliberal discourse which validated and rationalised it (Shamir, 2008; O'Malley, 2009). lyengar (1989) studied the societal effects of arguments for the disbandment of social services, finding that a prevalence of discourse punishing dependency on social services had profound effects on individual attitudes towards societal problems. Encouraging an emphasis on the individual as the primary locus of responsibility for protection from harm had the convenient effect of deflecting attention from its causes. Garland (1996) characterises the increased displacement of responsibilities onto individual citizens as 'a new form of governance-at-a-distance.' Similarly, D. Barnard-Willis and D. Ashenden (2010), who looked at the United Kingdom's recent efforts to frame online identity management as an individual responsibility, characterise responsibilisation as a mode of 'directing of conduct'. Surveillance studies scholar Mark Andrejevic (2005) describes responsibilisation as an evolving strategy of governance that encourages self-management and self-policing through technologically enhanced 'peer-to-peer' monitoring tactics and tools such as online social networking platforms.

Discourses and processes of responsibilisation figure prominently in current frameworks for privacy regulation. The Notice and Choice paradigm is a prime example. Solove (2012) characterises the Notice and Choice paradigm as a form of 'privacy self-management,' whereby users are, in effect, tasked with both the job of understanding the consequences of choosing platforms such as Facebook, and with protecting themselves from subsequent harms of their data collection practices on their own. Upon the first use of a commercial service, a user is presented with 'notice' in the form of a Terms of Service agreement (TOS) enumerating the privacy policies, rights, and responsibilities governing the platform, with 'choice' provisioned through a small checkbox at the bottom for a user to signify consent to or denial of the terms. However, the 'notice' provided by TOS agreements cannot guarantee meaningful transparency to an individual user. For example, an individual user will rarely learn that governments requested access to their data, as the disclosure of this information is supervised in secret court rulings (Facebook, 2015a). Nor do TOS guarantee that as a company's business model finds new uses for its users' data, what was previously protected won't now be exploited (FTC, 2011). The most consistent element of Facebook's privacy policies is just how often they change to take advantage of new business 'use cases' for data (Hill, 2014). Thus the notice to users provided is vague or limited, while at the same time setting forth the expectation that the user has been educated enough to now make decisions in their best interest. 
As for 'choice', though the Notice and Choice paradigm of consent appears to offer users a form of empowerment through the provision of choice, a user's denial to the stated terms precludes further use of the platform. This is a false choice in an environment where no alternative exists aside from 'opting out' (Barocas and Nissenbaum, 2009), especially since opting out may not be seen as a choice by human rights activists, for whom popular platforms such as Facebook are critical to reaching broad and target audiences in their work. Further, the act of providing consent in one isolated instance grants easy access to personal data to 'third parties' 'downstream' (Solove, 2006), much of it covert and unknown to the user, such as in the sweeping up of user data by local law enforcement, by globally-operating intelligence agencies (Meyer, 2014), or in its sale to 'data brokers' (Ohm, 2010). Consent given through Notice and Choice thus becomes an 'artificial procedural justification' offering a 'wild' card to companies as to what can be done with the data now and in the future (Ausloos, 2012). That Notice and Choice in actuality provides very little 'notice' or 'choice' means this paradigm of privacy protection merely provisions a pretence of control. Having provided content to a platform to use data in both known and unknown ways, users who want 'instrumental privacy'-which we define as an assurance of confidentiality of personal information on platforms such as Facebook-are left to take practical steps that may result in varying efficacy. Capacity building efforts such as digital security trainings, which aim to increase the efficacy of privacy practices, must also respond by compensating for the false promises and opaque statements that are embedded into different platforms models of privacy protection.

Because the Notice and Choice model is used so widely in commercial platforms, it follows that its use has an effect on the privacy of many different kinds of users, including those engaged in human rights activism. The roots of this model may help to explain its failings while also raising flags in regards to how it may shape norms outside its initial sphere of influence. Notice and Choice can be understood as the product of the United States' particular conception of consumer privacy, heavily influenced by commercial interests and built on a case-by case basis in courts, with limited regulation by bodies such as the Federal Trade Commission. The loosely governed approach in the United States is often contrasted with the European Union's more systemic framework, which employs the concept of 'data protection,' as laid out in the European Data Protection Directive (Directive 95/46/EC) in 1995. Despite the EU's protectionist framework, with its granular data collection and processing guidelines and restrictions, both conceptions have roots in the Fair Information Practice Principles of the 1970s (Solove, 2009), and the EU framework continues to rely on the Notice and Choice paradigm. This is significant, considering that the EU Data Protection Directive is increasingly looked to as a standard for countries outside the EU to emulate (European Commission, 2014). 
Applied within Facebook, Notice and Choice serves to reinforce the culture and practices of the company. Facebook's origin is in an experimental website called Facemash, created by Mark Zuckerberg in 2003 to rank the looks of students with ID photos stolen from a university database. It was soon shut down due to outrage over Zuckerberg's exploitation of this data and his disregard for consent (Harvard Crimson, 2003). In his next experiment, Zuckerberg developed the first prototype of the Facebook platform as users know it today. Facebook's TOS and interface have since undergone countless permutations, but Zuckerberg's ideas of privacy and online identity have continued to loom large. In 2010, Zuckerberg now famously told the author of the Facebook Effect (Kirpatrick, 2010) that 'you have one identity,' and 'the days of you having a different image for your work friends or co-workers and for the other people you know are probably coming to an end pretty quickly.' Five years since he made this statement, Facebook's 'real name' policy remains intact, though harms to vulnerable users like human rights activists have been well documented. Today, Facebook's user base has expanded far beyond the platform's original intended audience of college students with the majority of its one billion plus users today residing outside the United States. Some of these users are involved in social movements and human rights activism and these uses are also far outside the experiences of Facebook's creators. As one women's rights activist we interviewed put it: 'It's really not a tool built for activism and yet we use it, because we were on Facebook first and then people decided they wanted a revolution, so they organised through there.' [2]

Facebook's application in ways not anticipated by its creators produces the sort of 'frictions' discussed by Anna Tsing (2005) in Friction: an Ethnography of Global Connection. For Tsing, the global movement of ideas, people, and goods produces global paradigms that transcend local discussions while obscuring their origins. Though Facebook and the Notice and Choice model have a 'global' reach and impact on privacy norms-in that their uses circulate through transnational market flows, federated communications protocols, and fibre optic cables connecting the world-this does not tell us what effects they have on the privacy of people in any particular context (Nissenbaum, 2009). It follows that we can learn about the impacts of technologies and legal frameworks by documenting the frictions that result as these frameworks and technologies are applied in unanticipated ways in specific contexts. In the following section, we examine how the Notice and Choice paradigm and its use by Facebook shape the experiences of a particular group of human rights activists. This case highlights some of the most widely observed challenges in developing strategies for privacy and security in human rights activism which resonated across the 40 interviews we conducted, while also illuminating new directions for strategies and remedies. In light of privacy and security concerns and in agreement with the activists we interviewed, we have determined that the only way to share their stories is by removing names, geographical details, and other unique identifiers. 


\section{Threats, in Context}

As part of our Security in Context research project, we interviewed and facilitated a workshop on privacy and digital security with 16 women's and Lesbian, Gay, Bisexual, Transgender, Queer, Questioning and Intersex (LGBTQI) rights activists. These activists, representing several different organisations who work together through an informal network, described how, over the course of nearly a decade, their work has become increasingly dangerous, to the extent that they now face significant personal, organisational, and network-wide risks in continuing to pursue it. About a year before our interviews, one of the organisations received an anonymous threat in a message sent on Facebook, to cease work on women's rights and LGBTQI issues or face having their office bombed. Soon after, members of a nationalistic group assaulted several of the women's and LGBTQI rights activists physically at a protest. Additionally, around the same time, anonymous harassers began using one of the organisation's media materials and personal Facebook photographs to create videos claiming the activists were responsible for destroying traditional family structures through their focus on women's rights. The misinformation campaigns often included messages culturally equivocating the words 'gender' and 'LGBTQI' with 'paedophilia'. For the activists, there was no clear way to respond:

They used my photos, they exposed my personal contacts, and we couldn't reach the creator of the video because it was done in an anonymous way. We asked ourselves, what are the next steps we need to do? [3]

These campaigns and threats of violence appeared to emerge from an organised collusion between nationalist groups and governmental actors, part of a larger effort to marginalise women's and LGBTQI rights said to be motivated by the brokering of an important economic union with a much more economically and culturally powerful neighbouring country. The network of women's and LGBTQI rights activists heard from several sources that this neighbouring country had sent officials to train local media in how to wage these campaigns, as part of a broader effort to exert their cultural influence. The network felt these campaigns were successful in changing public perceptions in a way that has impacted their ability to safely continue to push for women's rights.

Amidst these developments, the network gained information that the neighbouring country was also tapping into phone lines and Internet Service Providers and tracking social interconnections visible through online social networking platforms. Concern and anxiety over surveillance and intrusion were inflamed by stories of hacked websites and email 
accounts, strange sounds heard when using Skype, and the presence of clicking noises when using the landline telephone. Though nearly impossible to know whether such phenomena are clear indicators of active surveillance, it was at least reasonably plausible given the consistency of more overt surveillance incidents such as regular visits and phone calls from local security agencies inquiring into the whereabouts of specific members. While this surveillance was a burden in its own right, there was also no proof that it was directly connected to the described attacks and misinformation campaigns; thus it was very difficult for the network to really define the source or sources of these threats and behaviours.

Before the violence and threats of violence began, the network felt a strong public presence, and thus a visible online presence, was vital to the success of their activism. The activists used their 'real', legal names in Facebook profiles, not just because Facebook's TOS states that users must do so, but because their profiles served as a public point of contact for those interested in joining their advocacy work. However, since the women's and LGTBQI rights network could now anticipate that a public presence and publicly organised actions might lead to more violence and harassment, they felt a need to use pseudonyms, and to generally be able to shape their identities as they saw fit. Facebook's rigid 'real name' policy became a clear point of vulnerability. They were thus forced to violate the policy in order to protect themselves.

Facebook's changing photo privacy settings also exposed the activist network to harm. Despite vigilance over privacy settings, personal photographs would find their way into new misinformation campaign videos. Upon having time to sit down and pinpoint the source of the leak, the activists found that the settings controlling the visibility of photographs had again been changed by Facebook. The harassers exploited this change to obtain new materials for their campaigns. After this incident, many activists simply deleted sensitive photos rather than risking further exposure. The activists learned to review their Facebook privacy and account settings on a regular basis due to this incident, but were still shocked to discover over the course of a workshop provided by Tactical Tech that once again, photographs previously visible only to friends had unexpectedly become 'public' without any actions taken on their part. Instead, this change could be attributed to Facebook itself. 


\section{Responding to Threats}

Despite the unexpectedness of the threats and attacks-and their heavy toll-shared experiences of harassment and attacks brought the activists closer. Several individuals and organisations came together to form a more cohesive network, out of a shared need to be more strategic in countering threats: 'Much of our understanding of safety is about reaching out to other people instead of relying on our selves. We need to be able to rely on community'. [4]

The network agreed to make public appearances together from this point forward, and they implemented a new office-wide physical security policy. They also set up a secret Facebook group (Facebook, 2015b) in order to document security incidents such as online harassment and attacks at protests. For the activists who administered it, the main draw of the secret group function was that such groups are only visible to explicitly invited Facebook users (Facebook, 2015c). This form of documentation allowed the network to quietly but collaboratively track patterns in their harassers' behaviours. It became apparent that the 'online' and 'offline' harassment they experienced shared a similar tone and language, which allowed them to establish links between these behaviours. One activist who spearheaded this effort described first hearing the idea of documenting security incidents during security training, about a year before the attacks. At the time, he couldn't find the immediate relevance of this advice, but the attacks changed his understanding of protection:

Before the attacks I felt it was more important for people not to worry than to know about threats [made] on Facebook. I was keeping secret from everybody when I was receiving threats on Facebook. I used to delete them. I thought it was protection if they didn't know. [5]

The use of Facebook secret groups, shows how the social network of activists facilitated joint ownership of security. Still, there was a clear friction here between Facebook's affordances and the activists' needs. Almost all those using the Facebook secret group function questioned the groups' true level of confidentiality. Though secret groups are invisible to uninvited Facebook users, the groups and the material they post is visible to Facebook and potentially to third parties who work with Facebook, as well as anyone else who gains access to the accounts of group members. A breach of any one account would expose the highly sensitive communication of the entire secret group. A fear persisted that even if information was not made public, opponents would find a way to get their hands on it: 'we are working in a secret group but how can we really know if it is closed or if some people can see our messages?'. [6] 
As part of an evolving security strategy, the network began to use fake Facebook accounts to track the discussions and plans of their harassers, which took place in open and closed Facebook groups. This tracking enabled the network to prepare for possible harassment and violence at future protests, but the tactic did not remain effective for long. After using fake accounts to track the planning of a new attack, the network reported these planned actions to the local police. When word of this somehow got back to their harassers, the harassers closed these groups and moved on to create their own new fake accounts and secret groups, thus avoiding further tracking from the network. This tactical dynamic, which Gary Marx (2009) characterises as one of 'neutralisation' and 'counter-neutralisation,' demonstrates the constant evolution and interplay of 'online' and 'offline' monitoring, harassment, and violence, which resulted in the women's rights and LBGTQI network spending an increasing amount of time monitoring their harassers through social media, fixing privacy settings, and learning new security practices. Activists in the network said they reached out to Facebook in order to report the nationalist organisation they linked to online harassment, threats, and violence at protests. In response, Facebook sent them automated responses confirming the receipt of their report. Meanwhile, despite their efforts, the collective was not able to mobilise local law enforcement to interact directly with Facebook on their behalf.

It is clear from the limitations of both the network's individual and collective efforts that the load of responsibility the women's and LBGTQI rights network carried for their self-protection was too great to bear on their own. Though addressing privacy and security collectively proved enormously helpful in regaining strength and becoming more strategic in their activism, the network was continuously compromised by both Facebook's rigid identity management guidelines, frequently changing privacy policies, and general opacity. In their opinion, Facebook has not been accountable for the terms the company itself sets out in its Statement of Rights and Responsibilities. As a result of their experiences, the activist network has feedback they want to deliver to the company. They believe they deserve more of a response to threats and attacks than an automated reply, and that for the sake of their protection, they should be able to define and tweak their identities through the use of pseudonyms or other self-defined forms of identification. Though Facebook recently clarified that the 'real name' policy requires an 'authentic identity' rather than an actual legal name, the fuzzy, arbitrary distinctions between these signifiers only add to the opacity of the guidelines (Facebook, 2015c). Activists in the network also believe Facebook should make clearer the true extent of privacy and confidentiality offered by the secret groups, in which content is not kept secret from Facebook itself. Finally, members of the network point out that in the same way that they've now installed physical security cameras in their office to alert them to changes in their environment, Facebook should alert them more visibly to changes in privacy settings which affect the relative anonymity of their sensitive, personal information. 
As for other structures that might be engaged for support, the network believes governmental actors should contribute to their protection. It remains unclear how direct a role their own national government played in their harassment and attacks, though evidence gathered by the network points to some sort of cooperation. One interviewee expressed scepticism in regards to the ability or intent of large supranational human rights structures to provide any concrete protection, due to a lack of economic incentives: 'the UN will not protect us. Human rights in this country are just about money'. [7] Meanwhile, the network felt that while local police were helpful as a form of protection at local protests, they showed no interest in investigating the links between 'online' and 'offline' attacks in a sustained manner. Finally, when we're told that there is a recently created cybercrime division in the national government to guard against new 'cyber attacks' and to push for more 'cyber security,' it is with an anxiety over what effects the accompanying new forms of surveillance or enhanced monitoring might have on their work: 'National security is one thing. Personal security is another'. [8] Clearly there is no single, cohesive governmental response that can address digital privacy and security on Facebook or across platforms. Rather, there are confusing, heterogenous structures for the activists to navigate, which in itself adds to the burden of their responsibilities.

\section{Remedies}

The experiences of the women's rights and LGBTQI network point to the need for a range of remedies. Those we discuss here relate directly to the perceived needs pinpointed by the activist network as they relate to the confidentiality of the messages they share with each other using Facebook. This discussion is informed by the high probability that many activists will continue to use Facebook, in spite of Facebook's shortcomings as a tool for human rights work. Of note, alternative online social networking platforms such as Diaspora and Crabgrass have existed for years, however, they were not used by the women's rights and LGBTQI network at the time of the attacks. Though Tactical Tech supports and promotes the use of alternative, open source tools and platforms, we also recognise the difficulty of 'migrating' to them from their commercial equivalents and thus we seek to mitigate the harms that can result from their use, while also demonstrating alternative options. The question, in light of a continued reliance on Facebook, is what ways exist to increase the amount of control available to users. This control becomes crucial for overall security, as the attacks on the women's rights and LGBTQI network showed that breaches in privacy can be linked with instances of physical violence.

In order to control access to all content in Facebook accounts, users may choose to add 'two-factor verification' through a Facebook feature called Login Approvals. This 
feature provides an added layer of protection against weak or compromised passwords by requiring the use of a security code sent via sms to the user's cellphone in order to log in to the platform. Unfortunately this feature does not help with the confidentiality of communications once access to the account is gained or if messages are accessed through the account of one of the other correspondents engaged in conversation. To guarantee confidentiality of the messages themselves, users may turn to the use of CryptographyBased Access Control Tools (Balsa, Brandimarte, Acquisti, Diaz and Gürses, 2014). The use of such tools can enable a user to take back some control over the level of confidentiality of their content, to the extent that a platform like Facebook, or potential opponent who gains access to a Facebook account, would no longer have the ability to read one user's messages to another.

Many of these tools, such as the 'chat clients' Jitisi and Adium, are free to use and readily available for download. Unfortunately, as Balsa, et al point out, they lack wide adoption. The experience of the women's rights and LGBTQI activist network is illustrative of some of the challenges of implementing such tools, helping to explain their low adoption rates. While the usability of tool interfaces has gained increasing attention in its importance as a barrier to tool adoption, we turn now to an interwoven but distinct issue that can override the usability considerations of the tool interface. Human rights activists are often motivated to master difficult tool interfaces. However, even when they do, through trainings or individually, they often have no one to use them with. To exchange encrypted messages, they must then take on the additional responsibility of spreading skills among colleagues or members of a wider network and in convincing them of the value of such digital security practices. Often this skill transfer doesn't take place because of constraints on time and resources, so that tool uptake remains limited to individuals with skills received via training or self-directed learning. This limitation points to a great need to more easily integrate digital security practices into the interactions of individuals and groups, both by optimising tools for use within groups and by facilitating more opportunities for learning within groups who are already engaged in communication with one another through online channels. As one interviewee told us, 'when it is about security of colleagues, the security becomes real'. [9]

Currently, the use of communications-based encryption is contingent on an exchange between two individuals, but for more than two people, this form of encryption can become exceedingly difficult to implement. In tools such as Jitsi and Adium, this is partially due to the specific cryptographic protocol implemented within them-called Off The Record Messaging (OTR) - though there is work being done to create a 'multi-party' version of OTR (Goldberg and Ustaoglu, 2009). In addition, Adium and Jitsi also cannot be used with Facebook's secret, closed, or public group utilities. Thus, these tools cannot mitigate the vulnerabilities created by the network's reliance on secret groups for group 
communications. The betterment of these tools will depend on how tightly technical specifications and cryptographic implementations are bound to the consideration that security emerges through interactions within groups.

Though this section has focused on the use of a particular form of encryption, we should note that the use of this encryption constitutes just one type of digital security practice, one which is not always appropriate for a user's given situation. The use of encryption offers an incomplete form of protection and can additionally constitute a form of exposure: its use can sometimes draw attention to activists in repressive environments if they have their electronics inspected or confiscated, or if certain activities are monitored online. Additionally, the use of OTR within commercial platforms does not stop the metadatainformation about who is speaking to whom, at what time-from being collected by Facebook or subsequently exposed to anyone who gains access to a user's account. We thus see that digital security practices dependent on currently available tools can only offer a piecemeal form of protection, even when implemented with careful consideration. We note that tool improvement is difficult and slow moving in an environment that favours commercial technologies, thus explaining why Free and Open Source Developers have not been able to solve the above dilemmas despite years of dedicated work to fix shortcomings.

\section{Conclusion}

The women's and LGBTQI rights network we have discussed in this article highlights the importance of social structures for privacy and digital security-with regard to both strategies and technical tools. That this network had to respond to threats in such a self-reliant way reflects a forced responsibilisation for digital technology users who want some protection of and control over their privacy and digital security. This network does highlight that building and sharing collective security strategies can contribute to the efficacy and longevity of activism, while also helping to lessen the burden of responsibility in learning to mitigate the harms of digitally mediated threats. Nonetheless, the resource differential between this network and their harassers-some of which are governmental actors-was enough that no matter what actions the activists took, they continued to face significant and unpredictable threats. Digital security strategies cannot remove all threats; they can only mitigate their effects. The strong social ties between individuals and organisations in the women's and LGBTQI rights network helped facilitate the continuation of their activism despite a multitude of threats, demonstrating a necessity for the human rights sector to brainstorm, develop, and support group-centric approaches to privacy 
and digital security. We argue that since group-centric approaches to privacy and digital security can help increase overall levels of efficacy and protection, such approaches should be prioritised in the creation of strategies, policies, and tools developed by and with computer scientists, advocates, and policy makers, within the human rights sector and beyond.

\section{Notes}

[1] Anonymous \#1 / Location redacted (2014). Interview with Becky Kazansky, Location Redacted, 11/2014. Unpublished transcript.

[2] Anonymous \#2 / Location redacted (2014). Interview with Becky Kazansky, Location Redacted, 11/2014. Unpublished transcript.

[3] Anonymous \#3 / Location redacted (2014). Interview with Becky Kazansky, Location Redacted, 11/2014. Unpublished transcript.

[4] Anonymous \#4 / Location redacted (2014). Interview with Becky Kazansky, Location Redacted, 11/2014. Unpublished transcript.

[5] Anonymous \#5 / Location redacted (2014). Interview with Becky Kazansky, Location Redacted, 11/2014. Unpublished transcript.

[6] Anonymous \#6 / Location redacted (2014). Interview with Becky Kazansky, Location Redacted, 11/2014. Unpublished transcript.

[7] Anonymous \#7 / Location redacted (2014). Interview with Becky Kazansky, Location Redacted, 11/2014. Unpublished transcript.

[8] Anonymous \#8 / Location redacted (2014). Interview with Becky Kazansky, Location 
Redacted, 11/2014. Unpublished transcript.

[9] Anonymous \#9 / Location redacted (2014). Interview with Becky Kazansky, Location Redacted, 11/2014. Unpublished transcript.

\section{Acknowledgements}

An enormous thank you is due to the groups who shared stories highlighted in this article, as well as to various staff and friends of Tactical Tech for supporting this research and for providing critical feedback on earlier drafts.

\section{Biographical Note}

Becky Kazansky is Lead Programme Researcher at Tactical Technology Collective. Through research and facilitation, she supports the development and implementation of privacy and digital security strategies to defend and extend human rights.

\section{References}

Andrejevic, Mark. 'The Work of Watching One Another: Lateral Surveillance, Risk, and Governance,' Surveillance \& Society 2.4 (2005): 479-497.

Ausloos, Jef. 'Guidelines for privacy-friendly default settings,' (KU Leuven, 2012): 34, https://www.cosic.esat.kuleuven.be/publications/article-2297.pdf

Balsa, Ero, Laura Brandimarte, Alessandro Acquisti, Claudia Diaz and Seda Gürses. 'Spiny CACTOS: OSN Users Attitudes and Perceptions Towards Cryptographic Access Control Tools,' Internet Society. USEC '14, 23, San Diego, CA, USA (2014), https://www.Internetsociety.org/sites/default/files/02_2-paper.pdf

Barnard-Wills, David and Debi Ashenden. 'Public Sector Engagement with Online Identity Management', Identity in the Information Society 3.3 (2010): 657-674.

Barocas, Solon, and Helen Nissenbaum. 'On Notice: The Trouble with Notice and Consent,' 
Proceedings of the Engaging Data Forum (2009) http://www.nyu.edu/projects/nissenbaum/ papers/ED_SII_On_Notice.pdf Baumer, Eric, Phil Adams, Vera D. Khovanskaya, Tony C. Liao, Madeline E. Smith, Victoria Schwanda Sosik, and Kaiton Williams. 'Limiting, Leaving, and (re)Lapsing: An Exploration of Facebook Non-Use Practices and Experiences', CHI Changing Perspectives (2013).

Bickert, Monica. 'Explaining Our Community Standards and Approach to Government Requests' (2015), http://newsroom.fb.com/news/2015/03/explaining-our-community-standardsand-approach-to-government-requests/

Bigo, Didier. 'Globalized-In-Security: the Field and the Ban-Opticon', in Sakai N, Solomon J (eds). Translation, Biopolitics, Colonial Difference (Hong Kong: University of Hong Kong Press, 2006): 109-156.

Braman, Sandra. Change of State: Information, Policy, and Power (Cambridge: MIT Press, 2006): 130.

Citizen Lab. 'Communities at Risk' (2014), https://targetedthreats.net/

De Wolf, Ralf, Rob Heyman, and Jo Peirson. 'Privacy by Design Through Social Requirements Analysis of Social Network Sites from a User Perspective' (2013), https://www.utwente.nl/bms/steps/research/colloquia_and_seminars/colloquia/bestanden/2013-2014/ pierson2013.pdf

European Union Commission. 'Commission Decisions on the Adequacy of the Protection of Personal Data in Third Countries' (2014), http://ec.europa.eu/justice/data-protection/document/international-transfers/adequacy/index_en.htm

European Union. 'Directive 95/46/EC of the European Parliament and of the Council on the Protection of Individuals with Regard to the Processing of Personal Data and on the Free Movement of Such Data,' 24 October (1995), http://ec.europa.eu/justice/policies/privacy/ docs/95-46-ce/dir1995-46_part1_en.pdf

Facebook Inc. 'Community Standards' (2015a), https://www.facebook.com/communitystandards

Facebook Inc. 'Government Requests Report' (2015b), https://govtrequests.facebook.com/ country/United\%20States/2014-H1

Facebook Inc. Facebook Help Center. 'What are the privacy options for groups?' (2015c), https://www.facebook.com/help/220336891328465

Federal Trade Commission. 'Facebook Settles FTC Charges That It Deceived Consumers By Failing To Keep Privacy Promises' (2011), http://www.ftc.gov/news-events/press-releases/2011/11/facebook-settles-ftc-charges-it-deceived-consumers-failing-keep

Garland, David. 'The Limits of the Sovereign State: Strategies of Crime Control in Contemporary Society', The British Journal of Criminology 36 (1996): 445-471.

Gellman, Barton and Ashkan Soltani. 'NSA Infiltrates Links to Yahoo, Google Data Centers Worldwide, Snowden Documents Say', The Washington Post, October 30 (2013), http:// 
www.washingtonpost.com/world/national-security/nsa-infiltrates-links-to-yahoo-googledata-centers-worldwide-snowden-documents-say/2013/10/30/e51d661e-4166-11e3-8b74d89d714ca4dd_story.html

Goldberg, lan, Berkant Ustaoglu, Matthew D. Van Gundy and Hao Chen. 'Multi-Party Offthe-Record Messaging', CCS'09, 9-13 November (Chicago, IL: 2009), http://www.cypherpunks.ca/ ${ }^{\sim}$ iang/pubs/mpotr.pdf

Gürses, Seda. 'Attitudes Towards "Spiny CACTOS"' (2014), http://vous-etes-ici.net/?p=80

Hankey, Stephanie and Daniel O'Clunaigh. 'Rethinking Risk and Security of Human Rights Defenders in the Digital Age', Journal of Human Rights Practice 5.3 (2013): 535-547.

Hill, Kashmir, 'Facebook Added 'Research' To User Agreement 4 Months After Emotion Manipulation Study', (2014 June 30) http://www.forbes.com/sites/kashmirhill/2014/06/30/ facebook-only-got-permission-to-do-research-on-users-after-emotion-manipulation-study/

Human Rights Watch. 'With Liberty to Monitor All: How Large-Scale US Surveillance is Harming Journalism, Law, and American Democracy' (2014). https://www.hrw.org/reports/2014/07/28/liberty-monitor-all-0 lyengar, Shanto. 'Framing Responsibility for Political Issues: The Case of Poverty', Political Behavior 12.1 Cognition and Political Action (1990): 19-40.

Kirkpatrick, David. The Facebook Effect: The Inside Story of the Company That Is Connecting the World (New York: Simon \& Schuster, 2010).

Marx, Gary. 'A Tack in the Shoe and Taking off the Shoe: Neutralization and Counter-Neutralization Dynamics', (2009) http://web.mit.edu/gtmarx/www/shoe.pdf

Meyer, D. 'Facebook Prism Case Heads to Europe's Highest Court' (2014 June 18) https:// gigaom.com/2014/06/18/facebook-prism-case-heads-to-europes-highest-court/

Nissenbaum, Helen. Privacy in Context: Technology, Policy, and the Integrity of Social Life (Stanford, CA: Stanford Law Books, 2010).

Notley, Tanya and Stephanie Hankey. 'Human Rights Defenders and the Right to Digital Privacy and Security,' In John Lannon and Edward F. Halpin (eds). Human Rights and Information Technologies: Trends and Consequences of Use (Hershey, PA: IGI Global, 2013): 157-175.

Pen America. 'Chilling Effects: NSA Surveillance Drives U.S. Writers to Self-Censor', (2013) http://www.pen-international.org/read-pen-american-centres-report-chilling-effects-nsasurveillance-drives-writers-to-self-censor/

O'Malley, Pat. 'Responsibilization', in A. Wakefield, \& J. Fleming (eds). The SAGE Dictionary of Policing (London: SAGE, 2009), 277-279.

Ohm, Paul. 'Broken Promises of Privacy: Responding to the Surprising Failure of Anonymization,' UCLA Law Review 57.6 (2010): 1701-1777.

Schwartz, Bari. 'Hot or Not? Website Briefly Judges Looks', The Harvard Crimson (2003 November 4) http://www.thecrimson.com/article/2003/11/4/hot-or-not-website-briefly-judg- 
es/

Shamir, Ronen. 'The Age of Responsibilization: On Market-Embedded Morality', Economy and Society 37.1 (2008): 1-19.

Solove. Daniel. 'A Taxonomy of Privacy', University Of Pennsylvania Law Review 477 (2006): 477-560.

Solove, Daniel. 'Privacy Self-Management and the Consent Dilemma,' Harvard Law Review 126 (2012): 1880-1903.

Stallman, Richard. 'How Much Surveillance Can Democracy Withstand?' (2013 October 14) http://www.wired.com/2013/10/a-necessary-evil-what-it-takes-for-democracy-to-survivesurveillance/

Tactical Technology Collective and Front Line Defenders. 'Security in a Box', (2015) https:// securityinabox.org/en

Tactical Technology Collective and Front Line Defenders. 'Towards Holistic Security for Rights Advocates’, (2014) https://tacticaltech.org/holistic-security

Tsing, Anna Lowenhaupt. Friction: An Ethnography of Global Connections (Princeton N.J.: Princeton University Press, 2005).

\section{OPEN HUMANITIES PRESS}

The Fibreculture Journal is published by The Fibreculture Journal Incorporated in partnership with Open Humanities Press. 


\title{
The Fibreculture Journal
}

DIGITAL MEDIA + NETWORKS + TRANSDISCIPLINARY CRITIQUE

\section{issue 26: Entanglements - Activis m and dechrology}

\section{FCJ-196 Let's First Get Things Done! \\ On Division of Labour and Techno-political Practices of Delegation in Times of Crisis}

Miriyam Aouragh

Westminster University

Seda Gürses

New York University

Jara Rocha

Bau School of Design

Femke Snelting

Constant Association for Art and Media

\begin{abstract}
:
During particular historical junctures, characterised by crisis, deepening exploitation and popular revolt, hegemonic hierarchies are simultaneously challenged and reinvented, and in the process of their reconfiguration in due course subtly reproduced. It is towards such 'sneaky moments' in which the ongoing divide between those engaged in struggles of social justice and those struggling for just technologies have been reshaped that we want to lend our attention. The paradoxical consequences of the divide between these communities in the context of the Internet have been baffling: (radical) activists organise and sustain themselves using 'free' technical services provided by Fortune 500 companies. While alternative technology practices, like those used among the Free Software Community, are designed, maintained, and actively used by a select few. We argue that even when there is a great
\end{abstract}


desire to bridge this divide, the delegation of technological matters to the 'progressive techies' reconfirms hegemonic divisions of labour and can be as pertinent to this gap as political and philosophical differences are. Conversely, if our tools inform our practices and our practices inform our tools, then we will have to reconfigure these divisions of labour between 'activists' and 'techies'.

doi: $10.15307 /$ fcj.26.196.2015

\section{Introduction}

This paper emerged from a workshop that formed part of the 2014 Thinking Together Symposium, held at the Osthang Architecture Summer School, Darmstadt, Germany. [1] It draws from conversations between the authors that highlighted how our approaches to brainstorming were eerily similar to the approaches of the tech-activist cultures we were trying to understand and challenge. We recognised that our pre-emptive questioning, pausing, and experimenting were mirroring the ongoing tensions between thinking and doing. Our own occasional utterances of things like 'Oh, lets first get things done!' made us realise we were all projecting from our particular-professional, political, personalcontexts. We came to recognise how we mimicked the same logic of what we identified and critiqued as a normative western male-dominated approaches that naturalise hegemonic divisions of labor justified by a quest for efficiency.

Our collective concerns are informed by narratives attached to: revolutions in the MENA region; uprisings in Gezi Park; the Indignados; the Free Software movement; and cryptoactivist communities. We share a curiosity about the pursuit of fundamental change that takes place at different political temporalities alongside techno-inventions. Time is never objective, and cannot be a standard empirical guideline, as our perceptions of time differ relative to personal struggles in a particular moment. The meanings ascribed to time for instance depends on moments of stalemate, sudden moments of suffocating urgency, fresh moments of new possibilities, and commitments to re-making that may span a lifetime, or longer. It is as if we are all placed within a matrix categorised by time and history; setting and locality; and technology, where each constellation brings another understanding of efficiency and urgency. 
At historical junctures, like the ones we find ourselves in now, hegemonic hierarchies are simultaneously challenged and reinvented. Sometimes they are aggressively imposed, but often they are subtly reproduced; this is what we have come to refer to as 'sneaky moments.' These represent controversial mass-mediated events such as leaked secretservice programs. What matters is that in these sneaky contexts, hierarchies are also reconfigured by deliberate technological interjections. We propose that the divide between those engaged in politics of technology and those participating in struggles of social justice are being reshaped during those 'sneaky moments' and we argue that this reconfiguration requires reflection.

But, where do we start? What are ways to resist the consequences emanating from sneaky moments that impose a pragmatic submission to the specialisation of work or delegation to experts? How do we identify the well-meant efficiency that reproduces hegemonic divisions of gender, race, class and age, and that reinforces ideological differences between activists for social justice and activists for 'just' technologies (Dunbar-Hester, 2010)? And, how can we prevent tech-activists from becoming coopted by policy makers with geopolitical interests like the ones we have evidenced in Internet Freedom projects in the Middle East (Ben Gharbia, 2010)?

We propose to begin identifying the problem through examples of mediation; investigating web-based campaign sites that claim to bridge the existing gap between social justice activists and progressive techies. Specifically, we look at sites that appeared or gained prominence at the onset of the public revelations about surveillance programs, as confirmed with the documents leaked by whistleblower Edward Snowden: our central sneaky moment. These campaign sites speak to the general public but especially encourage the use of secure communication tools for activists and journalists. But before we move onto this empirical analysis we first outline the critical reflections of activist use of media that have surfaced over the last years.

Our discussion explores responses to the Snowden revelations as relevant to the rise of campaign sites that promote secure communications. We further situate our analysis, the mise-en-scène, by introducing the actors we see as actively participating in the assemblage of activists and technology. Our objects of analysis are the campaign sites that promote secure communications. Through these sites, we discuss how matters of delegation and division of labour are configured. Finally, we express our ideas concerning possible ways through which to rethink and possibly contest these hegemonic modes of operation. 


\section{Delegation to Platforms and Delegation to Tech-Activists}

The process of what we call delegation of technical matters to commercial platforms has attracted the attention of academics and practitioners alike. The growing critique of the use of these platforms for social justice movements has various components, and has become the focus of important critical debates in communication and media studies that are relevant to our analysis. Companies like Facebook, Twitter, or Google are designed to maximise the possibility to communicate more, which, when used in the context of progressive or radical change, leads to an integration of counter-hegemonic political movements into the grids of communicative capitalism. These platforms serve to capture attempts at resistance through the seamless integration of political projects into the communication-entertainment complex (Dean, 2009).

Nevertheless, in moments of crisis, as people leveraged these technologies to establish new alliances for radical change, the platforms run by these multinational corporations were elevated in mainstream media to the status of 'liberation technologies' (Mejias, 2012). This also meant that the companies running these commercial social platforms were presented as gatekeepers of 'Internet freedom' [2].

The framing of corporate platforms as liberation technologies has overshadowed critiques of the profit agendas embedded in these monopsonies (Mejias, 2012). In the case of the Arab world, these kinds of projections have contributed to cyber-orientalism: the idea that 'traditional' people can be liberated by modern technologies, itself tied to a much longer history of essentialist representations of 'the orient' (Aouragh, 2015).

Other critics have argued that these platforms are sites for commodifying social labour, privatising social spaces, and subjecting dissenters to surveillance (Mejias, 2012, Fuchs 2009, Trottier 2014, Lyon 2008). The elementary functions delegated to these platformslike the management of content-make activist groups susceptible to practices of censorship and algorithmic organisation of content guided by profit logics.

Profit on these platforms is a function of the number of users and their levels of interaction. Crucial to this dynamic is the curation of a conflict-free social zone. Given the entanglement between user interaction, profit and conflict, the blocking of users and content-that may cause tension-has become a central feature of these platforms. 
Conflict management on social platforms, however, does not lend itself well to automation and requires expensive human labour. In order to contain the costs, conflict management disguised by the title 'content moderation' is outsourced to underpaid workers in Morocco or the Philippines (Chen, 2014). Paradoxically, delegating matters of technology to these platforms, counter-hegemonic activist groups become complicit with the labour conditions and logics of profit inherent to these commercial social platforms, that at the same time work to diminish their autonomy in organising their communications and actions.

The necessity of alternative socio-technical visions is evident when we consider the work that has been done to make other forms of technological organisation possible. [3] Some of these activities have culminated in what has become known as the Free Software movement. As early as 2008 the Free Software community responded to the threat of increased surveillance and loss of privacy (Franklin, 2008). In their view, the use of centralised network services had grave consequences for how the Internet, their most important working terrain, was developing away from 'Software Freedom' and freedom in general (Stallman, n.d.). Other groups, such as the Cypherpunks, focused on the development and dissemination of encryption based tools, which turned into a project of coding software that could 'make the networks safer for privacy,' and ensuring these technologies remain available to the public (Hughes, 1993).

While all of these groups are part of the story we are telling here, most important to our project is a small fraction of those progressive tech developers and advocates. Hereafter we shall focus only on those groups that have devoted themselves to putting in place infrastructures to protect activists from engaging in insecure communications. The activities of these groups gained momentum as popular knowledge about Internet surveillance and resulting government crack-downs increased.

\section{Surveillance Revealed: A Sneaky Moment}

The urgency of the moment around the Snowden revelations saw tech activists respond with a design proposal to reinstate privacy: a call to 'cryptographic arms'. According to this techno-legal consensus the revelations make evident that the United States government no longer limits its intelligence activities to military and diplomatic targets, but has exploited the 9/11 attacks on the World Trade Towers and the following War on Terror as a justification for engaging in mass surveillance across the globe. These efforts in surveillance have scaled so well thanks to advances in networked technologies and the dropping costs of processing and storage. This is both an economically informed enterprise and one that 
is based on an artificial difference between mass and targeted surveillance. However, the general public consensus is that mass surveillance is unacceptable as it reverts the presumption of innocence and violates people's privacy on the way to catching the 'bad guys', the subjects of targeted surveillance. [4] However, the shocking revelations regarding the NSA and GCHQ programs also undergirded the idea that if mass surveillance could be made inconvenient, somehow intelligence agencies would have to return to relying solely on methods of targeted surveillance. One way to make mass surveillance inconvenient is to make it costly. The idea is that if everybody, individuals and institutions, would use encryption, the cost of surveillance can be increased, stifling mass surveillance. The immediacy of the problem as well as the apparent absence of any effective transnational laws that could be leveraged against these surveillance programs, combined with the call to stifle mass surveillance, culminated in different campaigns to encrypt 'everything' on the Internet. While the main objective remained making mass surveillance expensive, encryption tools were paradoxically also seen as relevant for activists and journalists who were likely targets of intelligence gathering efforts.

These developments contributed to the momentum that saw numerous digital rights and Internet freedom initiatives seizing the moment to propose new communication methods for activists (and everyday citizens) that are strengthened through encryption. This was paralleled by numerous conferences, blog posts, press articles, online and offline actions, and, central to our analysis, the development and promotion of encryption tools that 'enhance privacy'. This also coincided with a somewhat ironic acceleration in the flow of funds from the United States Department of State, Silicon Valley and philanthropic foundations to digital rights and informational self-defence projects. [5]

It is at this juncture that the necessity and desire for a convergence between those 'groups that wish to use the media instrumentally to draw attention to their political efforts versus those who wish to change the media system itself' (Carroll and Hackett, 2006) became a matter of urgency. In response, a number of secure and private communication campaigns were launched or revamped, which also served to re-shape the delegation relationship between activists and this select group of technologists.

\section{Conceptualising the Campaign Sites}

Some theoretical considerations are worthy of mention here. A clearer understanding of activism in the context of technology and therefore also a better (progressive, critical) assessment of technology in the context of political change requires some effort to 
conceptualise the terms and labels we often use. Earlier we referred to two camps that have been labelled 'tech justice' and 'social justice' activists. While the emphasis on the two camps may suggest they are mutually exclusive, the social implications of the Internet for political mobilisation differ according to whom they cater to: activists for or by technology and activists with or via technology (Aouragh, 2012). Certain people do their activism with political change as the objective, and technology as the tool. For others, politics and justice is their context but a certain improvement in the tool itself is the objective. These overlapping identities and positions often shift or are part of parallel lives; in other words, some respondents in our respective research cases consciously divide between their techno-engagements for which they get paid and their other political work that they do for activist or ideological reasons and requires technological expertise. Changes in technology and technology governance, public space, labour conditions, and novel forms of organising also affect these divides and overlaps (Enrico De Angelis, 2015). Despite these nuances, our interest in this article is to draw out where these overlaps are negated, and to show how the divides are instated through socio-technical practices introduced in sneaky moments.

Another way of conceptualising our terms and labels is by deconstructing them, this is a similar but not identical exercise. For instance, with the Arab uprisings we learned that a proper assessment of the political implications of the Internet depends on two different characteristics of technology: as a tool for activists (operational, for example, coding or designing promotion material) and as a space for activists (mobilisation, for example, expanding networks, archiving) (Aouragh, 2012).

Furthermore, 'tech activists', far from a homogenous group, espouse a variety of political beliefs. These beliefs include attitudes towards design and how these matters translate to their activist practice. When it comes to gender, race, age, class and geography diversity among individual tech activists is less noticeable. This lack of diversity has been criticised from within and outside of the community. Certain civil society projects that produce campaigns can have a more diverse representation in gender, race, age, and geographical origin, for example, AccessNow or EFF. Still, those who are made prominent by virtue of a politics of representation that relies on viewership, tend to be white (Euro-American) and male, which is an important part of the re-instatement of hegemonic hierarchies between different activist groups.

Hegemony here is used in its vernacular meaning referring to dominance, but is also strongly linked to Antonio Gramsci's development and application. As Peter Thomas discusses-in what can be considered the most in-depth analysis of Gramsci-hegemony is a term that emanates from radical Marxist philosophy that has been disseminated via 
social scientists more than any other critical notion (Thomas, 2010: 133). Hegemony, in Gramsci's understanding, counters the false dichotomy between consent-based and coercive control. For Gramsci consent must be understood in its dialectical distinction to coercion. In constant interplay, consent and coercion figure as 'moments within each other, theoretically distinct but really united as moments of a political hegemonic project' (167). So hegemony is complemented (but not necessarily) by coercion (for example, violence) and in liberal democracies is often exposed through consent (for example, legal frameworks that are broadly accepted through media framings about its necessity) in the process contributing to the legitimisation of the state. Hence, both our understanding of power and dominance, and of sneaky moments, fold into the critique of hegemony, and necessarily so in light of the role played by security initiatives during particular moments as discussed in this paper.

\section{Rising to the Sneaky Moment of Surveillance}

Reflections on how the relationships between technology and social justice activists are being re-shaped is timely as we near the second anniversary of the Snowden revelations and the fourth painful anniversary of the MENA revolutions. In order to offer a critique, we studied several secure communication campaigns and analysed how they reveal their politics of mediation. While these campaigns exist alongside and in conjunction with training projects, we felt it was important to pay attention to the way these artefacts frame the relationship between tech activists and activists for social justice through language, selection, and design. From a large number of campaigns that are currently actively being developed, we selected three sites and deliberately included a non-anglophone example:

- Surveillance self-defence: Tips, Tools and How-to's for Safer Online Communications. [6] - The Guardian Project: Mobile Apps and Code You Can Trust. [7]

- Kem Gözlere Şiş: 'Bilgiyi şifrelemek, şifresini çözmekten daha kolaydır.' - Julian Assange ('Encrypting information is easier than decrypting information.' - Julian Assange. [8]

Each of these projects proposes to address surveillance in a different way. The material ranges from full-fledged software 'solutions' to PDF pamphlets; from authoritative manuals to quick guides for people in a hurry. Often recycling similar arguments, references, methods, and software choices, awareness of surveillance is raised on these sites through an ever-expanding ecosystem of activities. While in some projects serious translation efforts are made, we were especially interested to see if the more situated initiatives would provide design elements that mediated between spaces of action with a different understanding of divisions of labor. 
Before starting with a close reading and comparison of the way in which the content of the sites are communicated, we provide a description of the naturalised divisions of labour as enacted through the design, vocabulary, and other modes of address used in the campaign sites. Simultaneously, we look for conceptions of time and how the "urgency of the moment' is being mediated through these sites. We later use this close reading for a broader understanding of the performance of these sites.

\section{Devising Latent Structures}

The three campaign websites we survey are cultural artefacts, but they are also convivial spaces where various agencies co-habit with tools, discourses, and languages. In order to understand the dynamics of this complex scenography, we looked at the different gestures of delegation within these sites of mediation. By doing so, we started to identify some of the 'latent structures' that help us reflect on the situations in which these platforms function.

We argue that a dis-attention to the way the implicated agents (for example, the 'users', the 'developers', the tools, language and design elements) are mise-en-scène results in a division of labour that follows 'traditional scripts', and shows a perhaps un-intended hierarchy based on traditional models of production. As a result, their performance can take a tragic and unintended shape in which tasks and articulations of labour tend to echo fixed behaviours. Such hegemonically-scripted actions imply a strict management of expectations from all implied agents. They are often the result of top-down hierarchies, naturalised to the extent of rendering divisions of labour invisible. This is why we refer to them as 'latent structures': while they are certainly present on these mediation sites, they merge with the background and move out of our frame of attention.

This merging with the background is a product of, but also facilitates, the path dependencies that determine both tool development and use, and as a result all the agents pass through it without interpreting it as a structure. In other words: its smoothness is a tricky materialisation of a long period of hierarchical organisation through a hegemonic performativity. This smoothness also has to do with a cultural paradigm of "naturalizing the available' (Zapata, 2014). What this means is that agents perform within a pre-disposed framework, of which the limits, shapes, and shadows are no longer questioned, or may simply have found consensus: for example, in our case the assumption that a tool can provide security or enable anonymity. The reliance on available gestures is precisely the paradigm that helps merge latent structures to the background in our present (cultural, political) time. 
When our cultural-political ecosystems intensify or enter moments of agitation, then our relation to tools tends to fall into the paradigm of affordances. It does not matter how radical the political struggle is, people may succumb easily to work with the available. Dependency on the available plot of technological design is precisely what produces the conditions for a sneaky moment, at the risk of discarding very basic political, ethical, and aesthetic sensibilities. To make these residues of our sneaky moment more tangible, we turn to exploring the secure communication campaign sites.

\section{Designing the Divide Between Providers and Users}

The three campaign sites we have chosen are intended to mediate between the worlds of tech activists and social justice activists. We are interested in how they use language, design and tool-selection to bridge distances in knowledge, trust, and geography. If these projects are explicitly developed to communicate between agents that are not physically in the same space, how is a relationship of trust established? What do tech activists do to convince activists for social justice that they are on their side, and that the information and technologies provided are worth their trouble? And in the course of these relevant bridging and translation attempts, how do activists for social change find out if the provided tools are appropriate and safe for their situation? The three projects show similarities and also differences in their approach of how 'us' and 'you' are imagined.

A first thing to note is that both The Guardian Project (TGP) and Security Self Defense (SSD) establish a clear separation of roles between those that provide these secure communication tools, and those that should consider using them:

How to keep you and your communications safe wherever your campaigning takes you. [9]

Whether you are an average citizen looking to affirm your rights or an activist, journalist or humanitarian organization looking to safeguard your work in this age of perilous global communication, we can help address the threats you face. [10]

This seemingly simple construction of address effectively sets up a narrative where tech activists in-the-know are reaching out to others, activists for social justice that might need their help. Interestingly, Kem Gözlere Şiş (KGS) is more ambiguous about their perspective, and mix instances of 'us' and 'we/you': 
The objective of this project is to provide practical information about how to protect us from the 'evil eyes' that are watching us ... As your knowledge of the issues and your skills increase, you will see that you can better protect your personal data and your privacy, you will feel more powerful. [11]

Given the precarity with which KGS was created, these shifts in mode of address could be due to a lack of professional copy-editing or the inherent elasticity of the Turkish language. However, their consistent ambiguity does suggest that the KGS tech activists might sometimes get out on the streets themselves. The site continues to invite readers to become part of a community: 'This project aims to help users who need privacy, to provide tips on how to protect oneself from 'the evil eyes' and to create a growing community engaged in these issues.' [12]

SSD imprints its signature on every page by mentioning that it is 'a project of the Electronic Frontier Foundation' and linking to the EFF project page. The connection with EFF attempts to provide authority and perspective to skip any mention of 'us' in the About page. Rather, the About page of the SSD campaign starts with explaining the type of users this guide is meant for, before moving on to the project's goals and limitations. In a secondary menu we find 'credits' and a list of individuals that have contributed to SSD. While credits are given for specific contributions, nothing is said about the institutional or political affiliations of these contributors. A similar lack of attention can be found at the KGS site. Some limitations of the recommended technologies are mentioned but there is a vagueness about the identity of those who stand behind the site. TGP on the contrary has an extensive section 'About us' that explains 'How Guardian Helps', has all the individual team members listed with 'anonymised' pictures and provides extensive details about funding and affiliations, including a note explaining that certain United States government related funding has not co-opted their work. [13] In all three sites, through omission or over-exposure of contributors, the stages are set for the rest of the information to come.

The campaign sites build on the assumption that many activists for social change are not familiar with the proposed methods and tools, that this threatens their activities, and that mediation is needed to change that situation. One important concern is hence usability: security and usability are typically seen as complimentary, if not competing aspects of secure communication tools. According to experts in the field, usability in this context means that 'it is easy for the users [using the tools] to do the right thing, hard to do the wrong thing, and easy to recover when the wrong thing happens anyway' (Sasse and Palmer, 2014). 
Usability is addressed in two prominent ways. First, given that the usability of security tools is tightly coupled with doing the 'right thing' and, in moments of urgency, the 'right thing' is ambiguous for activists, it seems like a reasonable choice that SSD and TGP both employ 'scenarios' as the design method to communicate secure communication tools to their imagined or intended users. Through 'playlists' (SSD) or 'use-cases' (TGP) they categorise types of users according to their perceived security needs. In comparison, KGS does not provide explicit scenarios, but prefers to channel users' attention based on their devices: Mobile or Desktop. Scenarios in SSD are 'Activist or protester', 'Human rights defender', 'Journalism student', 'Journalist on the move', 'Mac user', 'Online security veteran', or someone who 'wants a security starter pack'. TGP on the other hand focuses on 'undercover human rights researchers', 'tech savvy citizen journalists' and 'activists in the streets', 'community organizations reporting on election issues', 'emerging online citizen journalists organizations', and 'mobile journalists for major news organizations.' [14]

Second, the three projects insist on communicating that the proposed technologies are easy-to-use. SSD literally offers a 'security starter pack' that is a manual which starts with threat modelling. TGP makes an effort with seven friendly icons ('So you got an Android phone?') each linking to an up-beat interactive explanation, consistently starting with 'Easy'. Both sites use design styles and language that mimic commercial on-line services. KGS evokes the 'security pack' with big red buttons suggesting one-click-install.

In usability design, a scenario is a description of a person's interaction with a system. It is believed that scenarios help focus design efforts on the user's requirements (Nielsen, 1993). The scenarios here though seem to directly map existing technological solutions onto supposed real-life experiences; they seem to be organised around assumed groups of solutions or technologies rather than in response to actual problems. An indication might be that most of the images and icons used on the three sites depict devices, rather than situations. Another issue that none of the proposed scenarios bring up situations that might ask for solutions not based on technological tools. The mantra of 'simplicity' hides the complicated and situated nature of using the promoted technologies in real-life situations, and designs away the human efforts that need to be made to put the advertised technologies into action.

From the awkward connections between technology needs and skills, causes and effects, devices and situations we started to wonder who was involved in the establishment of those categories. If these tech activists have already established relationships with activist groups on the street or in human rights initiatives to think through the required technologies and to test and develop them together, then the effort to communicate via a website is in fact redundant. On the other hand, if the projects are set up to actively 
reach out to unknown activists for social change, supporting a dialogue between groups is essential. SSD has a 'feedback' button on each page, but omits any possibility to ask for a return on the feedback. A secondary menu offers a standard contact form that is protected, but does not allow for any further secure forms of communication-for example, through email. The 'help us' that is repeated on most pages at KSG, includes the following rather conversational statement: 'We ask you to inform us about mistakes in any of the documents we provide. If you have suggestions for better solutions, please let us know. You can also contact us with questions about use.' Users are invited to send comments and suggestions by Twitter, indy.im, and Diaspora. When we first tried out the site, their email address had become invalid, which probably explains why the FAQ-which has great potential for communication-has remained empty. TGP states on their contact page 'If you'd like to learn more about Guardian from the team directly or have a proposal for us, please let us know using of the methods below'. The Guardian-Dev Discussion List is potentially the most interesting channel for user-provider exchange, as it invites developers as well as power users or 'just anyone interested in getting involved in the development side of things.' But even if this list may be read as the most inclusive of all three campaign sites, the listing of profiles centres again on technological development and negates any space for a collective exchange beyond this specific area.

By speaking to the activist audience rather than inviting them into a community of participation, the campaign sites in fact unnecessarily amplify the condition where activist communities are not expected to take part in the definition of the relationship they will have with the technologies they apparently need to depend on. Participation in activities that shape these secure communication tools is hard, but will be even less appealing (or even not known to the users) if not explicitly solicited. If problems appear, users will abandon tools that do not match their context. In moments of urgency, the drop rate may depend even more so on the urgency and needs of the activists rather than the positivist claims made on these sites.

\section{Connecting Technologies to Situations}

The activist may need to develop their own scripts of the possible ways in which tools can fill in roles during different moments of Internet activism. Currently, sites like Security Self-Defense by EFF, as is also typical in many cryptoparties, starts by asking users to do 'threat modelling'. As phrased on the website, 'To become more secure, you must determine what you need to protect, and whom you need to protect it from.' While threat modelling is a tool in itself that can be useful for activists, its military and industrial origins are ever-present. 
In professional settings-such as within a company or the military-threat modelling is assumed to be part of a number of activities conducted by a large team of developers: for example, when there is a bigger system development or maintenance project where security is one aspect among many. The language of 'assets' assumes that the owner of the system has (information) assets that need to be protected, and it is the security team's duty to make sure those assets are secured. This monolithic vision of a system to be defended is however outdated and criticised within security engineering. Even a corporate system is likely to have users and associates with conflicting interests, meaning that there may be numerous competing threat models, for different situations, the priority of which depends on the bigger project that is to be achieved (Pfitzmann, 2001). Addressing the social and political complexity of threat models would allow the SSD site to show that security is a negotiated process, instead of a clear state of affairs which experts can discover depending on their adversaries' capabilities.

Further, all the examples in SSD focus on information assets and relevant data, which may or may not be of primary importance for activists. Hence, the campaign site sees threat modelling through the perspective of the tools they offer: the tools are there to secure information, hence the activists should focus on securing information. This is very different than focusing on people, situations or political goals that may matter to the activists, which may or may not translate to 'information assets' afterwards.

Instead, all three projects claim, at least on the surface, to aim at the same type of users, under pressure of similar threats. It is in this context surprising how little overlap there is between suggested technologies. While both SSD and TGP are involved in developing software themselves, the first layer of each of the campaigns seems to focus on the curation of useful technologies, bringing them together in easily digestible 'playlists'. In these playlists-besides ChatSecure and Tor plus related software for phones, Orbot and Orweb-there is not always consensus between projects. This partially reflects the SSD's implicit tendency to focus on desktops and laptops, and TGP's explicit focus on mobile technologies, but the reasons for focusing on different hardware remain inaccessible to the targeted users.

What is also surprising, given that these sites are about security awareness, is that only SSD provides dates of the information that is presented. Campaigns may have starting dates, or the websites may contain reference to the year when the site was last updated; however, they lack prominent time stamps that indicate whether the information is fresh or 
outdated. Given that security vulnerabilities are disclosed every day, this leaves the users with the duty of checking whether these tools are still valid for use. The 'weatherrepo' federated app store is an initiative of The Guardian Project that hopes to provide an app store with vetted tools. While this is a timely project that could address the problem of continuous vetting, it is ironic that the weatherrepo information page states information about Yahoo! mail that is no longer valid. [15]

\section{A Wider Lens on Divisions of Labour}

The issues we highlighted so far point to an underlying division of labour through the distribution of roles according to expertise, in other words based on specialisation of work. The latter is most typically evident in the distinction drawn between developers and users. This division inevitably comes with expectations towards and assumptions about the capacities of each role. Foremost, it frames a dependency relationship and situates the expertise inevitably with the developer. In other words, the developer is seen to have the necessary and probably sufficient skills to develop a given technology (Suchman, 1994). Especially in matters such as security, where threats and vulnerabilities to the underlying protocols require extensive technical skills, this seems like a plausible delegation. But, is it?

For activists across parts of the Arab world, the conditions of their activism are increasingly being shaped by encroaching danger and enclosure. Rather than a widening of activist networks, maintaining their physical, social and political momentum has become priority. In this context, what an activist expects when organising or mobilising via the Internet (and mostly social media) is that it works. In working with these communities, our cautions about long-term risks involved with relying on commercial social networking platforms and our encouragement to engage in co-designing alternative infrastructures is at times met with bored sighs, pitying smiles, or confused stares. During a political tipping point, such as an uprising or the eve of a massive public occupation, users want efficient and ready-to-hand tools. Activists may not have the time or the patience to become designers too.

These statements are a residue of what popular commercial services are expected to offer-an expectation that normalises the delegation of numerous matters to developers and commercial platforms. Such unquestioned delegation confirms the latent structures that cement the power asymmetries between users and service providers. However, even in using these services, there is always some friction that is not captured by the developers, and here the activists hope that another division of labour within the 
movement will solve these: tech savvy activists can volunteer their time to making sure there is connectivity and that the tools work while others storm the streets. During tipping points, time is so valuable that it is not very wise to raise any question about pragmatic delegation decisions based on specialisation of work.

In the context of commercial platforms, this may come at the price of blocked accounts, adhering to real name policies, the frustration of having to manually recreate a friends list of a blocked account, or waiting for language features to be implemented: for example, Arabic hash-tags in Twitter. [16] These consequences affirm the motto that activists are opening their practices to change according to the tools that they use.

An engagement with alternative tech activists sensitive to their needs has the potential to reverse some of these dependencies, however, the campaign sites that we studied suggest that this is not guaranteed. While many of the secure communication tools are revolutionary in their protocol design, the campaign sites indicate that the same tools rely on very traditional framings of users and ways of relating to developers and technology. Expectations of a seamless service combined with inattentiveness to how the relationship is framed folds activist users and progressive developers into the available forms of delegation. This sets up the 'users' to oscillate between deliverance to developer decisions and disappointments with unsatisfiable expectations. In the pursuit of making complex encryption tools accessible to users in a format that they would recognise from commercial platforms, we find that the campaigns sites in fact amplify the user-developer opposition.

\section{Design from Nowhere in Service of Activists}

Focusing on usability pushes the task of integrating these tools into everyday practices onto the activist communities. It takes great amount of labour to integrate any new tool into the social tapestry that activists find themselves in. With every tool comes the laborious activity of configuring them to local needs, maintenance of tools on the variety of available devices, as well as the development of trust toward the tool developers through mechanisms like 'user support.' Given that these laborious activities are critical to secure communications, it is a concern that almost none of the campaign sites attend to these matters, and generally do not consider how people can move into the secure communication space collectively. In the pursuit of usable tools, the collective labour necessary to use them goes unrecognised. 
In that sense, these campaign sites that aspire to bridge between tech developers and activist users succumb to a commodification logic. The objective of these sites is to depict security tools as 'completed products.' Due to the lack of salience given to time stamping, the continued validity of the security and availability of the tools, as well as to the developer-centric modes of production comes to resemble a project of 'design from nowhere' (Suchman, 1994). According to Suchman, this is an ideal in which:

the goal is to construe technical systems as commodities that can be stabilised and cut loose from the sites of their production long enough to be exported en masse to the sites of their use (Suchman, 1994).

Subjecting secure communication tools to a commodification logic demands that security is a binary-you can download and be secure-a somewhat unachievable goal. This expectation pressures developers of secure communication tools to either come up with gross security claims or disclaimers that some of these tools may not work, pushing them to further confirm the illusion of a universe in which security exists as a binary.

This is a significant step away from the culture of secure communications. Practitioners participating in what can be called security design collectives will agree that 'it takes a village to keep a tool secure' and that security is a continuous 'cat and mouse game.' But this culture is lost on the campaign sites. With the exception of the empty FAQ on the Kem Gözlere Şiş site and the developers channel on The Guardian Project, there is little invitation on any of these sites that gesture at the idea of creating an activist community that can collaborate with the developers. This mode of mediation sets up the individual user 'to be the weakest link' instead of playing for the 'community to be the strongest link' in achieving a security aware activist culture.

\section{Universalising Tools for Global Users}

The objective of developing tools that can function across contexts is part of the universalist ideal typical of design collectives. Here, in the absence of far away users under threat, designers can invoke them at will and imagine their needs (Oudshorn et al., 2004). With the urgency to build secure communication tools that are easy to install and use independent of context, this practice becomes further normalised. Justified by a similar sense of urgency, some tech activists promote getting funding for a tool through, for example, Internet Freedom initiatives focused on 'dissidents in repressive regimes', which 
can then also be used by activists in so-called democratic countries. These pragmatic and equally troubling steps lead to many contradictions. For example, it makes it possible for campaign sites to reach out to users across the world, while the devices that are required to install the proposed tools assume users have access to some of the latest in mobile technologies and infrastructure.

Security design collectives also have the additional objective to make sure that the security guarantees of the tools that they develop are 'exogenous, homogeneous, predictable, and stable, performing as intended and designed across time and place' (Orlikowski, 2007). These tools are however entangled in very intricate political and social realities which technologists can only 'design around' to a limited degree. Nevertheless, in security engineering practice, the ambition to develop tools with universal security properties is seen as an ideal and is pertinent to modes of thinking that allow engineers to abstract away from the situated knowledge of a specific context and to shift real world problems into the technical solution spaces. This means that developers can pursue goals like developing an anonymous communications service like Tor, which provides anonymity or the ability to circumvent censorship regardless of contextual constraints.

Yet, while the design of anonymous communication networks is a challenge in itself and validates the need to abstract messy realities away, user community input can be almost as pertinent. In fact, the Tor community has been very aware that Tor can only work if they take situated reporting into account. When the act of using Tor, which can be identified by ISPs, can be sufficient to put a person under suspicion, it becomes evident that contextual realities matter and the design of tools has to be rethought. [17] In other situations local conflicts cannot be designed away and activists already under suspicion may simply be better off staying away from these technologies. This situation was expressed most articulately by Anne Roth, when her family was put under heavy surveillance in Berlin, Germany for unsubstantiated terrorism charges. Similar conditions hold for many members of the 'Muslim community' in the United States and Western European countries, who do not necessarily have the luxury of securing their communications, as this would trigger greater surveillance and suspicion. Technology design requires focusing on the security of activist interactions on the network, but other variables may creep up in unexpected ways. This requires tech activists to continuously revise their social, political as well as technical assumptions. As a consequence, it is very challenging, if not undesirable, to rely solely on technical experts to develop technologies for activists.

The heavy reliance on tech activists also conceals an effective international division of labour which all of the campaign sites affirm. What Mike Hales put into words in 1994 about corporate engineers still proves to be true in the context of progressive tech developers: 
Our times present us with a de facto economic and cultural separation between production and use. In our work world, producers are professionally (i.e., culturally) specialized; to a large extent, system-production is located in specialized and distinct sectors and/or geographical locations within an international division of labor (Hales, 1994).

In addition to the tendency for tech activists to develop universal technologies with a 'design from nowhere,' much of the development work occurs in the Western hemisphere where market values of efficiency and resulting coding practices are the rule. As more and more tech activists succumb to the pressure to develop ready-to-hand tools, this also means they are expected to replicate designs whose success is based on market parameters. This is the point at which most of the campaign sites and tool developers evaluate their success on the number of downloads or number of individual users rather than the efficacy of the tools for the projected activist communities. Once subject to these parameters of market efficiency and its correlated principles of design, questioning of hegemonic divisions of labour can only be regarded as counter-productive and inefficient.

A related anxiety among activists is that technologies tend to shape their environment towards increasing individualism (one of the features of social networking) and that this hinders collective action. A telling example is given at the Fourth Arab Bloggers Meeting in Amman (January 2014). This challenge was voiced during a heated debate about ways to bridge the gap between knowledge and practices of activism, more precisely how blogging can shift from an individual act to a more comprehensive collective performance. The Internet motivates micro-celebrities and social media stars, and this tendency is further triggered by traditional media where some bloggers and activists are put in the spotlight, treated as if they were spokespersons for the entire movement (Angelis and Della Ratta, 2014).

In an interesting reflection during the conference by a Yemeni activist, we are reminded that the dominant or expected hierarchies of priorities cannot be universalised. The person in question mentioned that social media trainings often don't take into account the needs of local societies, especially in terms of anti-surveillance programs:

Circumvention is not a big issue here; yet we heavily invest in training on such tools in every single event, conference, and gathering held in this region. Rather than the currently very hyped issues of cyber security/anti-surveillance, finding a safe offline space to meet and plan is of a much greater concern (quoted in: Angelis and Della Ratta, 2014). 


\section{Let's First Get Things Done: Modes of Operation for Sneaky Moments}

We suggest that one way to better situate secure communication development activities is to move from an attitude of 'design collectives' at the service of (individual) users, to 'designing for activist collectives'. This could be bootstrapped by avoiding inscribing users into the language of threat modelling, and instead inviting security engineers to step into the language of collective action within a political project.

For example, our experiences from the Arab world, Turkey and Spain suggest that the stage (or timing) of certain actions defines how relevant a tool is and what potential it may entail for a given action. A helpful way to think through these contradictions is to imagine a distinction between various stages of a revolution: pre-revolution (preparation and mobilisation); moment of revolution (the actual tipping points); and post-revolution (successful continuation or dangerous counter-revolution). It is useful to then juxtapose these historical timing-related factors with kinds of usage (sometimes as a space and at other times as a tool).

This distinction in time suggests that technology is not always the dominant driver of change but surely is one of the actors of change. This insight is relevant for understanding which tools, infrastructure and group-the activists for technology or the activists with technology-are best suited or should be more present at a given moment. Depending on these different phases, it may be more informative to rethink how the use of secure communication tools may be decisive (for early mass mobilisation), just mentionable (for class struggle) or virtually irrelevant (in military battles [18]).

Further, by framing the matter at hand in terms of the role of technology for activists in the context of the aforementioned pre/ during/ post categorisation of the political moment, it may be valuable to start by distinguishing what effect we expect technology to generate for political activists or politically motivated techies. Technologies may tip the scales of power when they help expand existing networks and thus become vital for the emergence of movements and campaigns. This can be achieved by interpreting the online/offline divide as a reflection of the space and tool separation, and this in turn as part of the overall political strategies and tactics without excluding any of the pre- or non-digital technological tools or spaces. 
Reframing the project of communications security as a constituent part of political activism may however still reproduce the user-developer dichotomy. A more radical proposal may be to shift this relationship by recognising that ultimately what is desirable is "collective design'. Here, the way in which Lorea, an alternative social network, proposed to frame the relationship between activists and technology provides some inspiration:

These networks are self-managed because Lorea is a non-profit, independent, open, and self-sufficient project. We don't talk of 'users' but rather of 'inhabitants' because we prefer a conscious coexistence instead of a simple, passive client relationship. Lorea inhabitants actively participate in the design, development, and maintenance of the network's working to implement the federation protocols, develop code, maintain safe servers, hunt down bugs, translate the interfaces into various languages, test user friendliness, document its development, and to undertake dissemination, help, or welcome activities for new inhabitants. There is thus no institution or formalized association behind Lorea, but rather a community of 'inhabitants' (N1Crew/SpiderAlex).

What is beautiful about the proposition of 'inhabitants' here is that it explicitly recognises the labour it takes to make a 'community tool'. Where Lorea probably had its shortcomings was in their inability to sustain the project and the costs of labour over time. For many tech activists, although not all, the dependency on wage-labour is something that they can free themselves from, at least temporarily (Soderberg, 2014). This ability to sustain oneself is, however, both gendered, raced and geographically constrained, if not also specific to the IT sector.

A focus on divisions of labour that defines roles based on their relationship to the software artefact leaves out the fact that the production, maintenance and use of the technology can only exist with the necessary sustenance of life, such as the production of food and shelter, for the reproduction of labour power. In many political collectives with their own space, this is also known as the problem of 'who pays the rent' and 'who cleans the toilets.' In fact, a lack of attention to matters of sustenance of life has been the breaking point of many alternative projects, if not the point in which corporate and government funding has found entry into alternative technology projects.

In talking about costs, it is important to circle back to the argument by tech activists that proposed an economic solution to end the NSA and GCHQ surveillance programs by raising the cost of monitoring through the use of encryption. We may sadly find that in this sneaky moment, the tech activists forgot to add to their equation the cost of integrating 
secure communication tools into the practice of social justice activists. In fact, the urgency of our post-sneaky moment maybe to think along the lines of class, and regional differences, as well as too easily assumed divisions of labour, if we want secure activist communication projects that truly scale.

As revelations about surveillance programs and related crackdowns on activists have emerged, tech activists grasped, remoulded, and redefined this occasion. They effectively translated what are considered surveillance problems into ones concerned with privacy and cryptographic self-defence. We have attempted to develop a vocabulary and offer a snapshot that could help us attend to the naturalised divisions of labour and the delegation practices of technology that manifest themselves between activists for social justice and activists for just technologies. In order to situate our discussion, we focused on the numerous initiatives that have emerged to provide activists with secure communication tools, seizing the momentum created by the anxiety about surveillance. Retrospectively, this turn happened almost naturally and invisibly, sneakily extending fringe secure communication tools to activist communities across the globe. The subtext in such initiatives invokes the notion of a universal user and in extension, that of a universal activist. This is where it starts to sound familiar; the hegemonic division of labour related to universalist ideas about technology results in concealing situated politics, interests, and contestations.

These projects make a lot of sense in a world where government and its services cannot be trusted. The proposed alternatives promise to protect activists from uninvited eavesdroppers and resulting vulnerabilities, while continuing to use Internet-based services. In doing so, the campaign sites channel the vacuum created by the revelations about surveillance programs into building trust around encryption technologies. They vet small tech activist initiatives for secure communications and vouch for their trustworthiness so that they can scale globally. Due to their sudden prominence, these sites also serve as hubs for delivering public trust towards Internet-based services. To rephrase: the sudden rise of these campaigns is not coincidental and for a variety of reasons have also found support from Fortune 500 Internet companies and governments scrambling to rebuild confidence in the Internet and associated markets (Wisniowski, 2012).

In summary, we have illustrated how these campaign sites transform tools developed by tech activists by delivering consensus around the use of the available structures for reframing secure communications technologies: for example, by depicting them as usable apps that are one-click away. In contrast to the careful articulation of politics through a diversity of tools, we found little attention given to the delegation relationship that is constructed between the invoked activists and the tech developers. 
The user-developer opposition reiterated on these sites gives insights into how specialisation of work and scarcity of resources can easily lead to divisions of labour, expressed across fault-lines of race, gender, class, age, and geography that are themselves already a consequence of neoliberal power. As a result, in these 'sneaky moments' tech activist communities and social justice activist communities, ideally a natural match, come to oppose each other. Our critique comes with its own risks. Given the positive valence associated with these campaigns and the largely marginal position of counter-surveillance initiatives, our critique may be seen as overburdening already contingent projects. Yet, we argue, given the impact and potential of these initiatives, critique remains necessary.

\section{Biographical Note.}

Miriyam Aouragh is an anthropologist and Leverhulme fellow at the Communication \& Media Research Institute of Westminster University, UK.

Seda Gürses is a computer scientist working as a Post-Doctoral Research Fellow at the Media, Culture and Communications Department at NYU, USA.

Jara Rocha is a cultural mediator and a core member of GReDiTS/Objetologías research group at Bau School of Design in Barcelona, Spain.

Femke Snelting is an artist and designer, member of the association for arts and media Constant in Brussels, Belgium.

\section{Notes}

[1] Thinking Together Symposium-August 2014 http://www.osthang-project.org/projekte/ thinking-together/?lang=en

[2] Internet Freedom, U.S. Department of State http://www.state.gov/e/eb/cip/netfreedom/ index.htm 
[3] As the 'social' has become increasingly 'networked', several free software projects have tried to address (some of) the critiques of the way in which dominant companies began to shape social networks. These contestations on sites like RiseUp https://help.riseup. net, Mayfirst https://mayfirst.org, or Lorea http://p2pfoundation.net/Lorea are manifested through modes of software production and design proposals that are expected to enable novel performances of notions like politics, transparency, privacy, security, freedom, the networked social, and infrastructure autonomy. We are not impartial towards these projects or their ambitions. The Darmstadt Delegation came together on the basis of a shared experience of troubling differences in the politics, values and practices of 'activists for social justice' heavily using networked technology for their struggles, and of 'tech-activists' who struggle to develop progressive and alternative technologies. In conversation with numerous initiatives that have aligned around Backbone409 (Calafou, http://backbone409. calafou.org), interference (Amsterdam, https://interference.io/), transhackfeminist! (Calafou http://transhackfeminist.noblogs.org/), noisy square (OHM https://noisysquare.com), and the Internet ungovernance forum (Istanbul https://iuf.alternatifbilisim.org/), we are concerned that due to pragmatic decisions in times of urgency and lack of resources, these struggles may subscribe to divisions of labour that reproduce existing hierarchies and dominant discourses and practices.

[4] In this argument, targeted surveillance is bounced between either being legitimate, and hence not worthy of further discussion, or out of the scope, since technical solutions cannot withstand methods used by a keen nation state adversary targeting an individual, community, or country. Both assumptions are problematic. First, intelligence agencies do not make the distinction. Second, the artificial distinction forecloses initiatives that address how decisions are made to target people, communities, and countries and what targeting entails. Third, it reduces the argument to an economic one, that is, it is about increasing the costs of surveillance, depoliticising the topic matter.

[5] Below is a sample of some of the funding reports that have been flowing into digital security projects: DRL Internet Freedom Annual Program Statement for Internet Freedom Technology, http://www.state.gov/j/drl/p/207061.htm; Portfolio Assessment of Department of State Internet Freedom Program: An Annotated Briefing, http://cryptome.org/2014/09/ rand-Internet-freedom-attack.pdf; Digital Defenders, https://digitaldefenders.org; Knight News Challenge on Strengthening the Internet, http://www.knightfoundation.org/blogs/ knightblog/2014/6/23/19-projects-win-knight-news-challenge-strengthening-Internet/

[6] Surveillance Self-Defense (SSD) is a project by the Electronic Frontier Foundation. The aim of Surveillance Self-Defense is to teach people how to think about online privacy and security so that they can adapt their choice of tools and practices in an ever-changing 
environment. The project is framed as a defence against the abilities of modern technologies to eavesdrop on innocent people. Some of the proposed tools are developed by the EFF themselves. EFF is based in the United States and funded by individual donors, NGOs and some corporate support. https://ssd.eff.org

[7] The Guardian Project (TGP) is developed by an international collective of software developers embedded in the Free Software community. They observe that mobile technologies are important for communication and collaboration, but problematic when it comes to personal security, anonymity and privacy. In response, the Guardians actively develop software applications, software libraries, customised mobile devices and tutorials. The project is funded through donations from NGOs around Human Rights issues such as Free Press Unlimited and Tibet Action Institute, as well as the United States Government's funding schemes for human rights projects that are channeled through the Department of State and Radio Free Asia. The Guardian Project also receives support from software related companies such as Google, and from philanthropic foundations. https:// guardianproject.info/

[8] Kem Gözlere Şiş (skewers to evil eyes) is a project developed by members of Alternatif Bilişim (The Alternative Informatics Association) in Istanbul, Turkey. Resisting the 'evil eye' of surveillance, the project addresses users in Turkey to prevent them from bringing their security and privacy in danger through careless use of communication devices. Kem Gözlere Şiş offers a software selection and related manuals in varying degrees of detail. Kem Gözlere Şiş is a volunteer project organised by members and the activities of Alternatif Bilişim receive event based funding from various local and international NGOs. https://kemgozleresis.org.tr/tr/

[9] Security Self-defense, Landing page. https://ssd.eff.org/ Last Checked: November 2014.

[10] The Guardian Project, Landing page https://guardianproject.info/home/partners/ Last checked: November, 2014.

[11] Kem Gözlere Şiş, Landing page https://kemgozleresis.org.tr/tr/kemgozler/ Last Checked: November, 2014.

[12] Bilgiyi Şifrelemek, şifresini çözmekten daha kolaydır. Kem Gözlere Şiş, About this 
project https://kemgozleresis.org.tr/tr/kemgozler/

[13] "Note: this project has received small grants and sub-contract work from organisations (such as the Open Technology Fund) and research projects (such as Tor) that receive funding from the U.S. Government and other governments around the world. None of this funding has modified or shaped our development plans, and we would never, ever put any sort of backdoor or compromised component into our software based on this funding." https://guardianproject.info/home/partners/

[14] How Guardian helps https://guardianproject.info/home/use-cases/

[15] WeatherRepo https://guardianproject.info/code/weatherrepo/ The webpage claims that Yahoo! transfers mails in the clear, a statement that no longer holds since the company defaulted to a secure protocol for webmail in 2014.

[16] Here we are assuming the situation in which the infrastructure is totally destroyed, as is the case in the recent battlefields in Syria. In the same country, geolocating individuals, food sources, neighbourhoods are of interest to the Syrian regime, as well as US and EU funded NGOs. When information about the location of bakeries and food queues turn out be important for attacks by the regime and intelligence gathering by foreign governments, information security can indeed be very relevant. (See for a sample of such surveillance http://caerusassociates.com/wp-content/uploads/2014/02/Caerus_AleppoMappingProject_ FinalReport_02-18-14.pdf)

[17] Twitter is now available in Arabic, Farsi, Hebrew and Urdu https://blog.Twitter.com/2012/ Twitter-now-available-in-arabic-farsi-hebrew-and-urdu

[18] See project on Tor Pluggable Transports, developed in response to increased use of DPI by Internet Service Providers to detect Tor users https://www.torproject.org/docs/ pluggable-transports.html.en 


\section{References}

Aouragh, Miriyam. 'Social Media, Mediation and the Arab Revolutions', Triple C Journal 10.2 (2012) http://www.triple-c.at/index.php/tripleC/article/view/416

Aouragh, Miriyam. 'Revolutions, the Internet and Orientalist Reminiscence', in Reem Abu Fadel (ed.). Visions of Tahrir: Connection Domestic and International Spheres in Revolutionary Egypt (London: Routledge, 2015).

Carroll, William and Robert Hackett. 'Democratic Media Activism Through the Lens of Social Movement', Theory, Media, Culture and Society 28.1 (2006): 83-104.

Chen, Adrian. 'The Laborers Who Keep Dick Pics and Beheadings Out of Your Facebook Feed', Wired, October 23 (2014), http://www.wired.com/2014/10/content-moderation/

Dean, Jodi. Democracy and Other Neoliberal Fantasies: Communicative Capitalism and Left Politics (Durham, NC: Duke University Press Books, 2009).

De Angelis, Enrico and Donatella Della Ratta. 'Mind the Gap: Bridging Knowledge and Practices of Activism' at the Fourth Arab Bloggers Meeting, Jadaliyya, June 7 (2014), http:// www.jadaliyya.com/pages/index/18040/mind-the-gap_bridging-knowledge-and-practicesof-a

De Angelis, Enrico. 'Introduction: The Hybrid System of Egypt and "Cultural Chaos"', Égypte/Monde Arabe, March 25 (2015), http://ema.revues.org/3398

Dunbar-Hester, Christina. 'Beyond 'Dudecore'? Challenging Gendered and 'Raced' Technologies through Media Activism', Journal of Broadcasting \& Electronic Media 54 (2010): 121-135.

Franklin Street Statement on Freedom and Network Services (2008), http://autonomo. us/2008/07/14/franklin-street-statement/

Haggerty, Kevin D. and Richard Ericson. 'The Surveillant Assemblage', British Journal of Sociology 51.4 (2000): 605-622.

Hales, Mike. 'Where are Designers? Styles of Design Practice, Objects of Design and Views of Users in CSCW', in Duska Rosenberg and Christopher Hutchinson (eds). Design Issues in CSCW (1994): 151-177.

Hughes, Eric. 'A Cypherpunk's Manifesto', 9 March (1993), http://www.activism.net/cypherpunk/manifesto.html

Mejias, Ulises A. 'Liberation Technology and the Arab Spring: From Utopia to Atopia and Beyond', Fibreculture Journal 20 (2012), http://twenty.fibreculturejournal.org/2012/06/20/ fcj-147-liberation-technology-and-the-arab-spring-from-utopia-to-atopia-and-beyond/

N1crew/SpiderAlex. 'Reclaim the Networks: Technological Sovereignty for Social Networks', https://n-1.cc/blog/view/76157/reclaim-the-networks-technological-sovereignty-for-socialnetworks 
Nielsen, Jakob. Usability Engineering (London: Academic Publishers, 1993): 99.

Orlikowski, Wanda J. 'Sociomaterial Practices: Exploring Technology at Work', Organization Studies 28 (2007): 1435-1448.

Oudshoorn, Nelly, Els Rommes and Stienstra, Marcelle. 'Configuring the User as Everybody: Gender and Design Cultures in Information and Communication Technologies', Journal of Science, Technology and Human Values 29.1 (2004): 30-63.

Pfitzmann, Andreas. 'Multilateral Security: Enabling Technologies and Their Evaluation', Informatics Lecture Notes in Computer Science Volume 2000, (2001): 50-62.

Sasse, M. Angela and Charles C. Palmer. 'Protecting You', IEEE Computer and Reliability Societies, January/February (2014): 11-13.

Soderberg, Johan. 'Reproducing Wealth Without Money, One 3D Printer at a Time: The Cunning of Instrumental Reason', in Stefan Meretz (ed.). Journal of Peer Production 4 (2014) http://peerproduction.net/issues/issue-4-value-and-currency/peer-reviewed-articles/reproducing-wealth-without-money/

Stallman, Richard, 'Who Does That Server Really Serve', https://www.gnu.org/philosophy/ who-does-that-server-really-serve.html

Suchman, Lucy. 'Working Relations of Technology Production and Use', Computer Supported Cooperative Work (CSCW) 2: 21-39 (1994): 21-39.

Thomas, Peter. Gramscian Moment: Philosophy, Hegemony and Marxism (Chicago, IL: Haymarket Books, 2010)

Wisniowski, Matthew. Engineers for Change: Competing Visions of Technology in 1960s America (Cambridge, Mass: MIT Press, 2012).

Zapata, Guillermo. 'Ni el Copyright ni el Copyleft te va a dar de Comer', El Diario (2014 November 13), http://www.eldiario.es/interferencias/copyright-copyleft-va-dar-comer_6_324127601.html

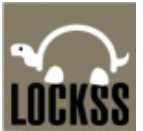

The LOCKSS System has the permission to collect, preserve and serve this open access Archival Unit

This Isuue of the Fibreculture Journal by The Fibreculture Journal Incorporated is licensed under a Creative Commons Attribution 4.0 International License.

\section{OPEN HUMANITIES PRESS}

The Fibreculture Journal is published by The Fibreculture Journal Incorporated in partnership with Open Humanities Press. 


\title{
The Fibreculture Journal
}

DIGITAL MEDIA + NETWORKS + TRANSDISCIPLINARY CRITIQUE

issue 26: Entanglements - Activish and dechrology

\section{FCJ-197 Entanglements with Media and Technologies in the Occupy Movement}

Megan Boler and Jennie Phillips

OISE/University of Toronto

\begin{abstract}
:
This essay explores the paradox of activists using corporate-owned platforms-the 'master's tools' (Lorde, 1984)-in the context of the Occupy Wall Street movement. Grounded in findings from interviews with 30 women activists from eight North American Occupy sites, this essay reveals the frictions that result from the entangled paradox between philosophies embedded within technologies and activists' philosophies. We document entanglements between corporate platforms and radical democratic ideals, and subsequent frictions between activists' ideals and more pragmatic, DIY practices. We also investigate frictions between aspirations of openness, and the realities of surveillance and infiltration by the police state. We examine entanglements through the theoretical lenses of 'connective labor' (Boler et al, 2014), 'veillance' (Mann, 2004), and the 'master's tools' (Lorde, 1984), and lay the groundwork for 'queering the binary of individuals and groups' (Barad, 2012) and recognising the non-linear, dynamic relations of social movements.
\end{abstract}

doi: $10.15307 /$ fcj.26.197.2015 


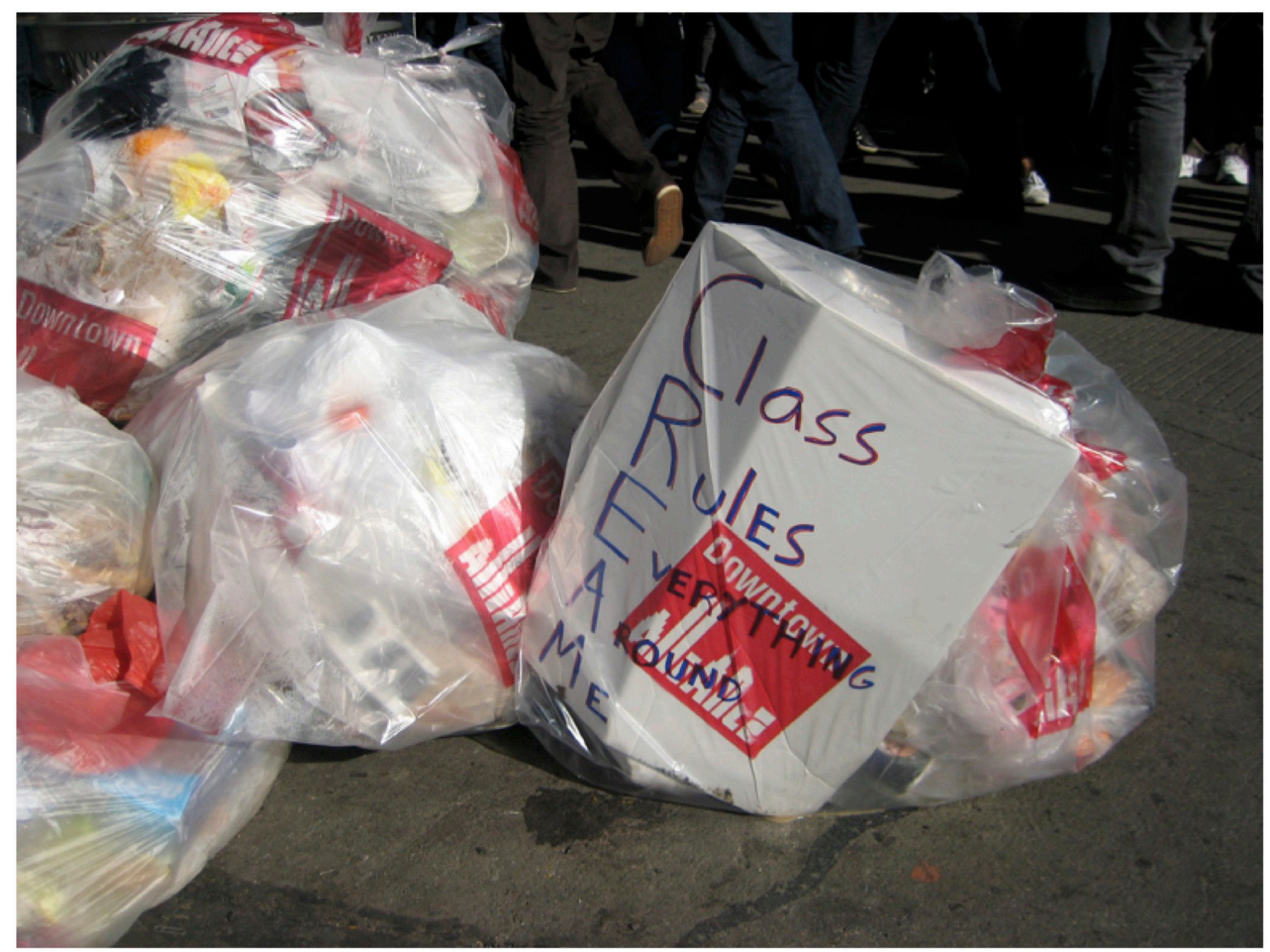

Figure 1: near Zuccotti Park, New York City, October 2011

(photo: Megan Boler)

\section{Introduction: Fighting Fire with Fire - Entanglements between Corporate-Owned Platforms and Activist Social Media Practices}

The digital era has seen activists around the world use social media platforms and information and communication technologies (ICTs) for social movement organising. Activist uses of corporate-owned social media platforms (from Facebook and Twitter to YouTube) and digital tools (including smart phones and digital cameras) support unprecedented coordination of local and global movements. However, these hybrid (online and offline) social movements [1] produce frictions that reveal discrepancies between the risks and promises of corporate-owned networks. This is certainly the case with social movements concerned with economic inequality, such as the Occupy movement, where such uses can benefit the very corporations the movement seeks to dethrone. Regardless of how one measures the roles and successes of social media in the context of activism, the uses of corporate-owned platforms and tools position activists within paradoxical entanglements between praxis and ideals. 
Questions about the efficacy of social media practices within social movements, and the roles they play in sparking and sustaining social movements are quite sharply contested (Gladwell, 2010; Shirky, 2011; Tremayne, 2013; Sigal and Biddle, 2015). Despite repeated dismissals of social media networks as merely 'weak' ties that have no lasting social change global uprisings and revolutions since 2011 increasingly many skeptics of social media's force, potential, and roles. [2] The application of these tools within the hybrid Occupy movement served many purposes. [3] Gaby and Caren (2012) demonstrate the role Facebook played in spreading Occupy messaging after protests started on September 7 , 2010, which included access to related news, information sharing, updates on meetings, and as a site for messaging. Posts about protests drew new members, especially those depicting images of confrontations between the police and protesters (Tremayne, 2013). The movement eventually spawned over 1500 Facebook pages, under names including 'Wall Street Occupation, local occupations across the globe, occupying specific institutions and networking occupiers' (Gaby and Caren, 2012: 267). These Facebook sites alone revealed four hundred thousand active users through Facebook pages. Activities ranged from sharing pictures, videos, to status updates (Pempek et al., 2008). Gaby and Caren (2012) report major uses of Facebook included recruitment of people and resources, information sharing, storytelling and 'access-group exchanges.' [4]

The Adbusters article, '\#OCCUPYWALLSTREET: A shift in revolutionary tactics' [5] (itself circulated largely online) is recognised for its key role in sparking Occupy Wall Street. The article describes how social media can contribute to 'shifting' or 'changing' the role of mass communication in social movements; that Twitter and other platforms can connect 'disparate peoples' with similar motives; and, that interactions are unpredictable between homogenous groups and even more so with heterogeneous ones (Adbusters, 2010; Tremayne, 2013). By using a mediated platform like Facebook, movements can enjoy a lateral mode of sharing, collaboration, and even coordinated action, given the connective nature of the communication technologies in use. [6] How and in what ways does this shift in revolutionary tactics play into the hand of the very corporations and their associated powers that a movement such as Occupy can be said to oppose? This paper explores this question through the qualitative findings of a three-year SSHRC funded research project that explored how young people use social media for community and movement organising. [7]

As we explore 'the entanglements that arise due to frictions between the philosophies embedded within technologies and the philosophies embedded within activism' (Shea et al, 2014), we identify three entanglements that emerge from our research findings:

- Entanglement 1: Women Activists' Digital DIY Practices: Accessing the Master's Tools 
- Entanglement 2: Using the Master's Tools for DIY Reporting: Frictions between Citizen Journalism and Corporate-Owned News

- Entanglement 3: Activist Ideals of Openness and Challenges of Infiltration and Police Violence: Subversive Uses of Master's Tools

Our essay explores entanglements as dynamic frictions; as the editors of this* *issue note, 'friction can be disruptive, productive, or both, and it may contribute discord or harmony.' Indeed our research underscores how the 'frictions between technologies and activists... ultimately enhance the ability of activists to take more control of their projects, create new ethical spaces and subvert technologies,' as well as 'result in tension, conflict and hostility' (Shea et al, 2014). By examining entanglements through the theoretical lenses of connective labor, veillance, and the master's tools, we illustrate how Barad's (2012) concern with 'queering the binary of individuals and groups' allows an account of social movements as dynamic and non-linear encounters and relations.

Occupy activists' ideals of fighting corporate oligarchies collide with their pragmatic uses of corporate owned digital tools. Our study revealed that the views of Occupy participants span both ends of the spectrum regarding this tension; from radicals and anarchists who insist that revolution is impossible so long as citizens divulge information through corporate social technologies; to pragmatists who advocate the necessity of reaching a wide audience effectively through 'free' services such as Facebook. These frictions rarely, however, prevent Occupy activists from using Facebook, Twitter, YouTube, and other corporate owned and widely accessible platforms. However, even in the few years that have passed since the heyday of Occupy movement camps and associated mobilisations around the globe, reluctance to use these master's tools has exponentially increased. The surveillance and infiltration made possible by state access to these open networks and communications, combined with high-profile cases such as Wikileaks and the Snowden revelations, has increased activist sensitivity to the risks of organising social movements via the master's tools.

\section{Quantum Entanglements and Implications for Social Theory}

'Entanglement,' the theme of this issue of Fibreculture is a rich concept increasingly used by those in humanities and social sciences, significantly as a result of interventions made by quantum-physicist and social theorist Karen Barad. Entanglements imply profound and unresolved paradoxes that challenge the realism and causality theories commonly embraced by physicists and many others. 
It is worth providing a short overview of the concept of quantum entanglement. In 1935 Schrodinger developed the term 'entanglement,' following correspondence with Einstein, who described the uncanny paradox of inexplicable communication between particles as 'spooky action at a distance'. In brief, when two originally independent and measurable entities interact - particles, for example-the state of the post-entanglement entities can no longer be understood, described or measured independently from one another. What can be approximately modelled post-entanglement is what is termed the 'quantum system'. But any attempt to measure one of the discrete interacting constituents in the resultant quantum system collapses the state of the entire entangled system. This paradox is particularly troubling to scientists wedded to a local realist view of causality.

Furthermore, even if one instead focuses on measuring the quantum system, at best a model can approximate but can never exactly measure the quantum entanglement under scrutiny. Karen Barad notes that this challenge, in a positive sense, "queers the binary of the individual and the group' (Barad, 2012b: 77). Within dynamic and non-linear quantum entanglements, scientists invoke the concept of 'hidden variables' as an explanatory solution to some of these unknowns-in short, the result of entanglements and the nature of an encounter which refuses our perception, knowledge, and measurement. Any description of entanglements necessarily requires admitting a substantial margin of error, and significant hidden variables, all of which underscores the challenge of predicting the result of interactions between entities. The significance of new materialism and agential realism (as Barad terms it) is the growing application of these questions and increasing acknowledgment of the radically 'unknown' intra-actions within socio-cultural and political systems.

As the notion of entanglements demonstrates, one cannot easily predict the results of an encounter between entities in a system or state-space. Will the use of open and publicly accessible information shared by activists via Facebook result in police infiltration and surveillance? How might women's often invisible, gendered connective labor change our entanglements with technologies, or create more inclusive social movement participation? What is the significance of women's increased access to participating and controlling the media(ted) means of production; of being interested to learn and adopt DIY skills to become Facebook admins, documenters, and connectors (Boler et al, 2014), using technologies often not previously used?

The digital tools used by activists provided a necessary medium for connecting nodes, but simultaneously the use of open and public platforms like Facebook and Twitter meant that the Occupy movement was exposed to additional infiltration from naysayers, police, and other undercover operatives set out to 'bring down' the movement (Kiley, 2012). Given the horizontal (leaderless) social movement structure and the Occupy movement's principled 
commitment to openness and transparency, the movement was especially vulnerable to surveillance.

\section{Background: the Hybrid, Horizontal and Leaderless Occupy Movement}

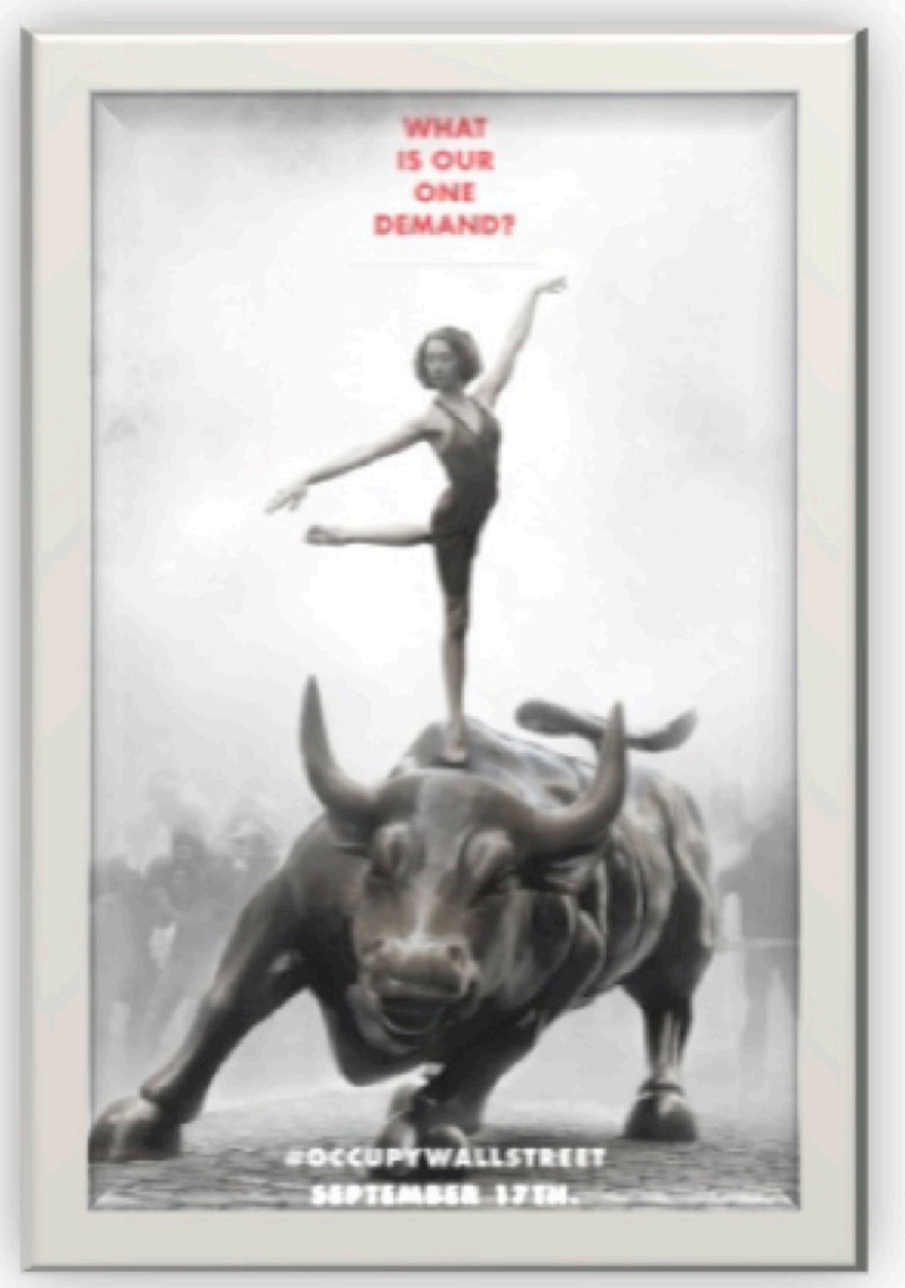

Figure 2: Adbusters' OWS call, 2011

Occupy Wall Street (OWS) began on September 17, 2011, in Zuccotti Park on Wall Street, the financial centre of New York city and site of the United States Stock Exchange. Responding to a call issued through the Canada-based magazine Adbusters, the movement was directly modelled upon and inspired by the Spanish social movement, Los Indignados. [8] 
Its representative meme 'We are the 99\%' captures not only an American national shift in awareness regarding wealth inequality, but rather a global zeitgeist. [9] On October 15, 2011, through globally orchestrated solidarity, Occupy Wall Street became an international movement with participants in more than 100 cities in the United States and 1500 distinct occupation sites around the world (Occupywallstreet.org; 2015).

Occupy adopted many practices engaged by Los Indignados and other contemporary as well as earlier social movements-from the occupation of public spaces, to large open meetings called General Assemblies (GAs). In general, the movement stressed an emphasis on process as being as important as the end goals ('Be the change you want to see!'). The diverse, localised yet globally united Occupy camps and cities protested corporate ownership and governance through unusually creative and radical approaches and processes. [10]

Occupy is recognised as a hallmark example of horizontalism, a consensus-based, leaderless organisation increasingly common in contemporary social movements. Elsewhere, we explore the significance of this emphasis on process which acknowledges that the process of making a better world is as important as the ultimate goal or vision of that world (Boler and Nitsou, 2014; Boler et al 2014; Ratto and Boler 2014). This shift from vertical hierarchical leadership structures to horizontalism is further linked to what we explore as the shift from collective to connective action (Boler et al, 2014; Bennett and Segerberg, 2012). Central to the connectivity that describes our social media networks, is what Bennett and Segerberg call the 'logic of connective action'; they describe such connective action networks as 'far more individualised and technologically organised sets of processes that result in action without the requirement of collective identity framing' (Bennett et al, 2013: 750). In contrast to both collective and vertical 'hierarchical coalitions of organisations,' Castells (1996) describes contemporary social movements as 'fine-grained, multilayered networks' (Bennett, 2012).

When our research team interviewed the first 50 participants of our study at the Occupy Toronto public encampment in November 2011, we were surprised that every participant interviewed-from the most radical anarchist to those who did not even own a mobile device-used Facebook for* all* communications related to Occupy and also as their primary filter and source of news. In the subsequent 30 interviews with female Occupy participants from nine different Occupy cities in the United States, $100 \%$ of those we spoke to used Facebook for most aspects of their political and social organising; however, they also frequently used platforms such as Twitter, YouTube, Vimeo, or other web-streaming platforms for the posting and sharing of videos and citizen journalism related to Occupy. [11] 
A significant feature of involvement in Occupy as well as in most contemporary movements includes low-barriers to participation. This refers both to the easy access via everyday technologies; the inclusivity of perspectives; and the use of 'accessible' slogans and ideologies, such as the $1 \%$ and the $99 \%$. That said, many of the key women participants and 'connective labourers' we interviewed were not significant users of digital media and technologies prior to OWS. Many did not have extensive resources and did not own a digital camera or even a smartphone, and hence it was often due to serendipitous acquisition of such technologies that women described becoming 'avid documenters'. For example, one participant began her connecting and documenting work only after her brother gave her a smartphone and added her to his cell phone coverage plan. Another received a digital camera for a birthday, which then allowed her to begin documenting. Another did not livestream until she realised what technological tools were needed and that they were not prohibitively expensive to access and use. In addition to accessing the tools were the skills needed to deploy them effectively for labor ranging from recording events, live streaming, uploading to a streaming site, or administrating Facebook pages. The demand to engage in connecting, documenting, and 'adminning' led to women learning, through DIY (do-it-yourself) practice, and by DIT (do-it-together) of watching how other Occupy participants used these ICTs effectively.

In 2011, there was not yet the widespread suspicion, public awareness and resistance to corporate plundering of our private data for advertising profit (Facebook opened to the public in September 2006; Twitter in July 2006; and YouTube in February 2005). Since then, public awareness of the risks of sharing data and the realities of surveillance is radically and rapidly changing. Efforts continue to develop and build alternative technology infrastructures that would move such communications off the grid of unabashed data-mining, invasions of privacy, and related risks of corporate and governmental surveillance (Deibert and Rohozinski, 2009; La Rue, 2013; Pillay, 2013; Deibert, 2013; Citizen Lab, 2014). This was one response to the surveillance of social media:

We were very, very open with the city, with everything we were doing. To this day, we are too horizontal. It's a problem, because sometimes we cannot get things done specifically how we want to. Sometimes you have to have some strategies. So, if you say you're going to be at this and this place at this time, when we show up, there's a bunch of police already (Katie, Occupy Los Angeles).

Alongside the risk of surveillance, our interviewees shared consistent examples of using digital tools and platforms to circulate visual documentation of police and state violence and repression: 
You could see how LAPD was [saying] 'okay, you guys stay here, oh okay, you go there' and they were, ... just kind of doing this ballet orchestration of [the media]. But two blocks away they're beating people and the media wasn't allowed to go over there and see that. And then when they shot the guy in the tree house, the media didn't show that. But the live streamers, (like) Magda, ... she live streamed it, shooting the guys in the tree house (Penny, Occupy Los Angeles, italics added).

This entanglement of police violence and sousveillance reflects the encounters and frictions as layers of intra-actions: between police and activists' video documentation; between corporate and citizen journalism; between human and technology, itself with complex layers of hardware, software, interfaces, human attention and energy. These entanglements expand the robust strength, reach, and capacity of social change movements, while also placing at risk the privacy (and often the ideals) of activists. Activists' use of corporate owned and surveilled social media platforms to document, organise and distribute counter-discourses to the ruling logic of capitalism reflects the paradox of 'fighting fire with fire'. In the case of Occupy, the ideals of fighting corporate greed are in direct friction with the pragmatic practices of utilising tools that benefit the very corporations participants seek to undermine. [12]

Our analysis of activist entanglements takes up the challenge suggested in a previous issue of Fibreculture:

The popularity and rich cultural experiences witnessed in these spaces cannot be simply dismissed as yet another form of corporate control over culture, or Orwellian dataveillant machine. It would thus appear that current analytical frameworks and tools have failed to fully comprehend the ontology of commercial Web 2.0. If we are to identify critical alternatives to commercial Web 2.0 and, more generally, if we are to intervene in the ontogenesis of Web 2.0, we need to reconstruct a critical approach that deals with these contradictions (Langlois et al, Fibreculture, 2009, emphasis added).

We intend this essay to outline how the entanglements illustrate the urgent need for 'critical alternatives to commercial Web 2.0,' and to provide a* 'critical approach that deals with these contradictions' *through theoretical concepts of veillance, connective labor, and the master's tools. 


\section{Conceptual Frameworks: the Master's Tools, Connective Labour, and Veillance}

Surveillance, and veillance more broadly, is infiltrating the everyday lives of people around the globe. The era of ubiquitous digital media ensures that this trend will not disappear. Whether used by police, states, corporations, activists, or other social change agents, veillance is rife, and includes the familiar practice of surveillance, as well as Mann's notion of 'sousveillance' or turning our cameras around to 'look back' at the surveillance powersthat-be. Veillance is enabled through use of digital tools and particularly cameras or other forms of capturing information and data.

This dialectical dynamic of veillance is captured by a concept we borrow from Audre Lorde to understand the participants' entanglements with technologies: namely, the 'master's tools.' We adapt her question, 'Can the master's tools dismantle the master's house?' to explore the paradox of entanglements between philosophical and political ideals and pragmatic needs for activists to reach the widest possible audiences using the master's tools.

In a renowned speech delivered in 1984, activist, poet, and writer Audre Lorde asserted that the 'master's tools can never dismantle the master's house.' Adapting Lorde's concept of 'masters tools', we use the term to refer to social media platforms such as Facebook and Twitter, which systematically profit from selling user's data and which enable surveillance by corporations and other interested parties including the state and the military; as well as to technologies produced for profit and to an extent for data gathering, such as smartphones and other digital recording devices. Women activists' of the Occupy Movement engage and repurpose the 'master's tools,' juggling the contradictions between strategic and pragmatic choices on one hand, against the surveillance risks of utilising corporate owned master's tools.

The entangled dilemmas of a veillance society and of creatively repurposing the master's tools, were encountered by the women activists we interviewed in the course of their connective labor: they organically established unique roles we have identified as that of the 'Admin' (Facebook Administrator), the 'Documentarian', and the 'Connector', roles enacted through women's uses of social platforms and tools including Facebook, Twitter, and live streaming. 
Following Barad's conception of entanglements, we explore frictions between corporate owned technologies and activists not as a binary but as a dynamic and productive tension. Connective labor, our third theoretical lens, reflects the embodied entanglement between and amongst humans (for example, activists and police, activists and media makers), and between humans and technologies (activists and platforms, or police and platforms). Within a social movement context, connective labor reveals the entangled mediations of both material, as well as less tangible realities and practices of veillance and the master's tools.

Throughout our interviews with nearly 30 women Occupy activists across North America and with 80 Occupy Movement activists in total, participants identified the value of 'subverting' various 'master's tools,' as this woman from southern California describes:

I became excited about media because of this movement, because it suddenly was a tool that was for the people and not just something that was gonna' be a watching, like something that's invasion of privacy. Suddenly it became a connector ... we turn that around and we flip it when what was private before, now is a public mobilisation tool and people are all becoming part of a movement because of a Facebook page ... we've subverted something that was potentially damaging. (Maggie, Occupy Orange County)

The appropriation and re-purposing of such tools reveals the anti-corporate ideals of activists in dynamic tension with the vying needs for pragmatic efficacy and affordances of both many-to many and one-to-many communication, despite the very real risks of surveillance.

As we reflect on the question of how to use the master's tools to dismantle the master's house, we can draw on the following remarks by network theorist Jussi Parikka:

The only effective form of intervention in this world is to learn its laws of operation and try to undermine or overrun them. One has to give up being a player at a fairground sideshow and become an operator within the technical world where one can work on developing alternatives. But this then begs the question: What are the skills needed to 'become an operator'? This question is at the core of media theory and 'what is media' that differentiates theorists: is it just technical skill? Is it conceptual? What cultural, contextual skills do we need to operate? This affects how we think of networks too. What is taken to be a network? (Soderman et al 2014; emphasis added) 
Parikka's call for us to become 'operators' resonates with the realities of women activists' DIY learning and action, and how they gained access to tools and social media in order to enact the three key roles of connective labor: the admin, the connector, and the documenter.

Our exploration of entanglements builds on our previous work which foregrounds the connective labor of women Occupiers who actively employ digital media technologies for movement building. In our essay 'Connective labor and social media: Women's roles in the 'leaderless' Occupy movement' (Boler et al, 2014), we identified women's key participation and gendered labor in the North American Occupy movements.

The embodied and material nature of women's connective labor has supported, and in many ways sustained, the contemporary Occupy movement. Connective labor involves maintaining fluid interfaces between the lived material realities shared in meetings, alongside web-based communications such as information sharing, dialogue, decisionmaking and strategising. Both online and off, connective labor works to ensure smooth interfacing and communications across and between the mediated sites of social movements, and calls for skills of meeting facilitation, inclusive and careful listening capacities, organisational facilitation and delegation-to name a few of the requisite skills for connective labor.

Connective labor is intra-action between humans and technology. These entanglements between humans, platforms, ideals and pragmatics reveal intra-action and the symbiotic relationship between materialities and meanings. 'The existence of a strong correspondence between matter, practice, and meaning in Barad's work (2007), indicates that 'matter' can be seen as inseparable from the actions it engenders. As such, separate worlds of, on the one hand, obdurate matter, and on the other, active practices do not exist' (Alberti and Marshall cited in Scott and Orlikowski, 2010: 12).

Entangled intra-actions reveal how 'materiality is understood not as a 'thing' or 'an inherent fixed property of abstract independently existing objects of Newtonian physics' (Barad, 2003: 822), but a process of materialisation that configures reality. Even concepts, rules, language, or software (which are often offered as examples of immateriality) cannot exist without material enactment-whether embodied in thoughts, produced in action, or expressed in texts, machines, or running code' (Scott and Orlikowski, 2010: 12-13). 
Occupy was further characterised by an inherent (and hybrid) creativity, arising in large part from the adaptation of (1970s, second wave feminism) organising tactics including horizontalism and consensus-based decision-making, and consciousness-raising practices. In that essay we foreground the experiences of women Occupiers who actively employed digital media technologies for movement building, and identified three key roles which ground our theory of connective labor: Facebook Administrator, Documentarian, and Connector. These roles distinctively exemplify and fit the definition of immaterial and affective labor (Boler et al, 2014; Boler and Nitsou, 2014). This connective labor that fuelled Occupy, as our interviews and research revealed, is highly gendered and remains largely invisible. While these three roles overlap, there are also important distinctions:

- Facebook Admins organise and publicise the efforts of their Occupy Movement 'city/site.'

- Documentarians archive and author.

- Connectors share, mobilise, reach out, and network.

Connective labor pays particular attention to immaterial and affective work, and builds on Bennett and Segerberg's (2011) 'personalised collective action' and 'logic of connective action.' Diversified self-production and distribution of media result in more heterogeneous, sometimes conflicting, visions of movement building. [13] These women activistshistorically excluded from access to means of media production and circulation-engaged various DIY and/or DIT modalities to learn from others or teach themselves to use ICTs and platforms ranging from Facebook administration to Twitter to live streaming. The women's adoption of these roles illustrates an extended enactment of Bennett and Segerberg's (2012) 'logic of connective action', revealing the often hidden connective, digital labor of women in sustaining the networked and affective dimension of social movements.

Women's uses of corporate social media networks to administrate, document and connect the movement resulted in fraught entanglements of exposure whereby the 'gaze' of power-particularly of law enforcement and corporate-owned mass-media, as well as both offline and online publics-functions as a perpetual surveillance, alongside the simultaneous empowerment and capacity building afforded by the same media (Boler et al, 2014). 'Veillance' (Mann, 2004; 2013) provides a conceptual approach 'to reconstruct a critical approach that deals with these contradictions' (Langlois et al, 2009). This meta-conceptual framework also provides an analytic approach for understanding the materiality of interface between humans and machines.

Veillance, as Mann defines it (2004), includes two key dimensions: surveillance and sousveillance. The reality of surveillance weaves through our everyday lives as a manifest 
practice whether we pay attention or not. Around 1 billion Internet users live in countriesapproximately 40-with regular censored Internet (Deibert, 2013). Surveillance, the watching from above, was brought into stark relief through international cases such as Wikileaks and more recently Edward Snowden's whistleblowing on the United States NSA violations of constitutionally guaranteed privacies.

Mann notes that 'sousveillance is often implemented by media that is controlled centrally, and thus subject to surveillance,' which points out again built-in contradictions and frictions. However, at the same time, sousveillance 'tends to be distributed and less organised, or at least less hierarchical, and thus conducive to a small community in which individuals trust one another' (Mann, 2004). Veillance is useful especially in its articulation of the dynamic system of surveillance in relation to sousveillance. The felt and material contradictions within the lived context of veillance reflects the dynamic, even dialectical, tensions driving contemporary social movements.

Those we interviewed did not express extensive worry about corporate invasion of privacy and access to profile data. However, in the context of Occupy-particularly when speaking with dedicated anarchists active in Occupy at local conferences-discussions would often turn to the problematic fact that the fibre optic systems not to mention the social media platforms used, are owned by those profiting from our use of technological hardware and software. In sum, complex entanglements emerge as activists engage corporate owned tools and platforms to counter capitalism, and to administrate, document, and connect the movement. The fraught materialities of exposure to the surveilling gaze of power (particularly police and law enforcement and corporate owned mass media) coincide with the charged sense of empowerment and possibility for these first time and veteran activists.

\section{Occupy Entanglements: 'Reclamation of Public Space' and 'Coming Up Against a Monster'}

Our world is not working for most people. ... I'm exceedingly inspired to be with a group of people who are thinking from all kinds of different angles about similar problems ... I do believe the state is afraid of us. They would not execute their violence upon us elsewise, right? Even though it seems like a losing battle as you see endless footage of police brutality and cops coming in and throwing tents in garbage trucks and whatnot. The reality is that people mobilising, exercising their very basic freedom of assembly and specifically the act of occupying space, bringing to light the fact that it's already occupied space 
and we're on indigenous land, but on top of that, I mean, when capitalism first began its journeys, there was still something called the 'commons' ... the reclamation of public space and the very weight of having people say 'no'-although we're coming up against a monster (Tyana, Occupy Wall Street).

We now address in more detail the entanglements that emerged within the course of our research, and situate these frictions within the context of connective labor of women Occupy participants who navigate the vicissitudes of veillance and the paradoxical uses of the master's tools.

\section{Entanglement 1: Women Activists' Digital DIY Practices: Accessing the Master's Tools}

In the formative weeks of the study, our interviewees reported access to the media tents being highly controlled; as Penny described, 'In the beginning I tried to join the media team but I was pretty much not allowed inside the media tents.' These tents were situated at each Occupy site-they were areas protected with tarpaulins containing tables and digital tools such as computers and locally established wifi networks. Our observations of these media tents revealed a gender division, as male Occupy participants frequented these spaces, while women tended to be in the spaces related to health care, food, or clothing. A fellow participant testified in agreement:

Yeah, there were people like Penny who wanted to get involved but it was so locked down, that we had a security tent within the tent (Penny laughing) and if you couldn't pass that security tent, you couldn't even get in. I mean you could go in the tent but you were locked in within another tent. It's just ... it was bad because we had so many talented people around the encampment that wanted to help but we weren't letting them in (Penny's friend and Occupy participant, Occupy Los Angeles).

During our interview in downtown Los Angeles in December 2012, a year after all of the Occupy camps had been closed by militarised and coordinated police efforts across North America, Penny describes teaching herself the skills of live streaming, engaging DIY methods to acquire skills to use diverse communication technologies. 
I was at GA every night with my little flip cam, I was filming GA and then I would go home and then I would upload it to YouTube, and then I would go to school the next day and then after school I would come back and go to GA and do that every single day. So I was producing more media then probably the entire media team but I wasn't really involved with that team and then in January I started live streaming. I probably would have launched it sooner if I knew I could of. Because I had looked into it but, I looked at live stream and it seemed like it was expensive, you had to pay for it, it seemed out of my reach, and that maybe it was too technologically advanced but then for watching live stream I realised that some of the streamers were using Ustream with android phones, I learned by watching how to do live streaming so in January I started live streaming. Now, I am working with different Occupy news, I am working with OWS week and Occupy public access TV in New York and then also different collaborations with different people (Penny, Occupy Los Angeles).

Penny's description reveals how she engaged DIY practices to become entangled with the digital tools in order to serve as a primary documenter and connector for Occupy. Her experience also shows frictions between activists (being excluded from generally male-dominated media spaces) and frictions between activists and technological access.

She worked 'under the radar' of her Occupy colleagues for some time in order to open yet more inclusive spaces of direct democratic participation within socially-mediated public communication such as one of the Twitter hashtags being used for OLA. Penny describes how she gained access to a Twitter hashtag that was being under-utilised and how she mobilised it as a major source for ongoing, live updates as well as providing anyone and everyone open access to this OLA twitter hashtag. This illustrates the multiple ways in which Occupy women self-skilled in order to assume more active roles in the movement. Social media, it turns out, is perfectly suited to job sharing in these new activist configurations:

Actually, from the very beginning I took over one of the twitters [accounts] by myself because we had ... OWS Los Angeles twitter and then we felt that was too long, so we got Occupy LA twitter so we were tweeting off of both of those ... there was a lot of strict rules about how we were going to run social media and I didn't feel like I could really follow the rules, so I kind of took the OWS Los Angeles twitter all by myself ... ran that one, and I probably tweeted a lot more than the whole team tweeted and I kind of just, well, 'fuck the rules, I don't like your rules, I am just going to do what I am going to do ... everything I am going to do is supporting Occupy LA but I am too restricted by the rules.' 
I even got to the point where I will write the password on the bathroom wall ... It hasn't been hacked or compromised ... nothing bad happened ... Want the password? (Penny, Occupy Los Angeles).

In our analysis of women's key roles within the movement, Penny's role emerges as that of both a connector and a documenter. Through her DIY efforts she learned how to use these master's tools, and her persistence saw her develop media capacities that had at first seemed prohibitive. The challenge of accessing the technologies and putting them into use is exemplified by her comment, 'I probably would have launched it [my livestream] sooner if I knew I could've.' This example saw a first-time young woman activist with limited cultural and economic resources appropriate tools to become the key livestreamer and documentarian of Occupy Los Angeles. Penny's persistence and commitment, her labor as both a connector and documenter, proved indispensable to the movement. This was echoed by a fellow activist, 'Penny has become our soul ... her footage is worth a lot' (Katie, Occupy Los Angeles).

As explained by another interviewee, her relationship to the tools is defined by connective labours-being a Facebook admin, connecting, and documenting. Her labour thus helps to magnify the voice of Occupy, encouraging a level of information sharing that served as a catalyst for other peoples' engagement.

I was totally technophobic ... but when the movement started I just started adding people and within, I remember, in nine days I had like 900 new friends ... and it's people from all over the world. What I saw in terms of the mobilisation over the Internet was fascinating and how everybody started sharing what was really going on in places, and how it has Google Translate right there, and I could read articles from everywhere in the world. People were sending stuff about what was happening in their city and it was the most worldwide news that was present and what's happening in some city in Hamburg, Germany, and what's happening ... in Algeria. ... I never would have jumped into it if the Occupy movement hadn't suddenly catalysed that ... (Mary, Occupy San Francisco).

Penny's experiences reflects a common experience of feeling excluded and alienated from accessing digital technologies, yet Occupy provided opportunities to catalyse women to engage DIY methods and overcome technology-related fears. 


\section{Entanglement 2: Using the Master's Tools for DIY Reporting: Frictions between Citizen Journalism and Corporate-Owned News}

Occupy participants engaged the master's tools and utilised sousveillance to counter misreporting, or lack of reporting of Occupy events. A persistent thread across all interviewees was the need to identify free spaces to amplify the unfiltered, uncensored, living reality that was Occupy. This interviewee addresses her use of social media to fight the dominant narratives of corporate and traditional news media:

I was trying to fight some of the media narrative because we have so much paid advertisement, paid media coming at us that I really wanted to educate people. And really connect with people too that were of the same mind because I can shout all I want as one person and you look maybe kind of like a crazy person but if you have a 100 people shouting about what they want then you're a force to be reckoned with (Anna, Occupy Santa Cruz).

Another participant explains the disconnect between what the media was reporting and what was really happening on the ground:

Tuesday? Oh Tuesday's the day ... [the police] were supposed to shut it down. ... it was the first time that a comparison started to happen where you started tracking what the real media was saying about what was happening and you could see the total 1984 Newspeak going on. You're like, 'what the fuck?' Because you're there. Your friends are there and then you're reading the articles and you're going, 'that's not what happened.' And you're starting to actually see this fallout, and it's not that... I didn't have any love of media before that point, but I really was like, 'this is crazy' (Maggie, Occupy Orange County).

Her description of traditional media reports on Occupy events as 'Newspeak' demonstrates a widely shared sense of disconnect and conflict between divergent narratives and discourses about Occupy. Significant here is that movement participants increasingly came to 'trust' or privilege eyewitness accounts, variations of citizen journalism as well as video of events quickly uploaded to video streaming sites. In this sense, one can identify the entanglement with the master's tools, which enabled participants to provide what they considered to be accurate, grassroots, and eyewitness media coverage. 
Aptly summarising these entanglements, one interviewee describes how:

This [Facebook] data feed felt like a total participatory action study ... In essence, by being out there on the front lines and getting news media right there at that time, it was like we had infiltrated and we were actually seeing the 'Newspeak' really happening ... We the people can make their own news now.

This same participant then describes sousveillant entanglements, with the Occupiers as the infiltrators who had a unique opportunity to witness newspeak being produced:

By being out there on the front lines and getting news media right there at that time, it was like we had infiltrated and we were actually seeing the Newspeak really happening. ... And it was the very first time that I ... kept the news that the media made and the news that we had created ... it's just really fascinating to me ... this whole media thing ... people are taking video of the cops beating up the people, and the cops used to be taking the videos. So it's like we've got this panopticon both sides. We're all watching each other now (Maggie, Occupy Orange County).

Maggie's description reveals the entanglement of veillance in action-the muti-directionality of cameras, using the master's tools to record police brutality and document the events not fully-reported by traditional news. The end result is what she describes as a dual-directionality panopticon.

One participant explains how social media tools allowed activists to challenge the mainstream media's narratives and document what was actually happening on the ground to show police brutality and the closure of camps:

And so, the night of that raid specifically ... there was a group of us on Facebook and people were getting Twitter feeds too, and at the same time we were getting information both from the regular media, we're listening to the regular media while getting news feeds from our friends, and we were putting everything, one after the other up on Facebook and just feeding, and we created our own newsreel, because we were getting in, this is what happened, and 'the cops just came up and now there's a standoff, and now there's'... So, it was interesting having five different people on our side taking in news 
straight from the front, from where the guys were and then having that running reel of what [mainstream media] were saying was happening and then putting both in...like, '[mainstream media] just said this, but look what we have' (Jean, Occupy Los Angeles).

Another participant from another Occupy site describes her connective labor, and use of these platforms for 're-reporting', using the master's tools to produce eyewitness news and filtered, or synthesised news as an alternative to the misleading traditional media coverage (or lack therefore) of Occupy:

I was doing that extreme information feed, six live feeds open at once, reading all the Twitters, reading several hashtags at once, and just watching them refresh and refresh and trying to figure out what was happening. ... I was re-reporting it in a slightly condensed (way) ... giving more of an update and overview with links so that people could hook into that story and know what was happening (Laura, Occupy San Francisco).

Engaging the master's tool to challenge the traditional, unidirectional forces of one-way surveillance (watching from above), the activist above describes the use of cameras and posting these alternative citizen reports as a subversion of the surveillant gaze: "people are taking video of the cops beating up the people.' This subversion of master's tools to watch the masters demonstrates sousveillance, or 'watching from underneath.'

Mann identifies sousveillance as an urgent focus for our practices, lives, and research:

Sousveillance, derived from French 'sous' (below) and 'veiller' (to watch), is the art, science, and technologies of 'people looking at'. Sousveillance typically involves small person-centric imaging technologies, whereas surveillance tends to be architecture or enviro-centric (cameras in or on the architecture or environment around us) (Mann, 2005).

Other participants describe the frictions between corporate owned reporting and front line documentation by Occupy activists with the master's tools, of events that remain unreported by traditional media: 
Only us that actually went through it and saw it, that actually were there, understand what happened, and that's why we make sure that we have our own people ... taking pictures (or) live streaming (Penny, Occupy Los Angeles).

In sum, the frictions between corporate owned news and DIY reporting by activists using the master's tools determine whose narrative is accepted as authoritative; and whose story 'counts' as the record of the entanglements between activists, police, and the state.

\section{Entanglement 3: Activist Ideals of Openness and Challenges of Infiltration and Police Violence: Subversive Uses of Master's Tools}

Our participants highlighted frictions between platforms and tools as potential apparatuses for surveillance that simultaneously afforded the capacity for outreach and organising. There were heated debates and differences amongst the participants of the horizontal, leaderless movement regarding planning and strategies, and around surveillance and infiltration versus the commitment to openness and inclusivity. The central use of Facebook and Twitter for communication, organisation, and planning meant that many of the social media conversations surrounding Occupy were open, transparent, and public. The encampments as well as the General Assembly meetings and working groups also followed along the same vein. While smaller planning groups met off camera and offline, the Occupy ethos of DIY culture prized openness and transparency, despite the corresponding risk of infiltration:

Everyone still says there's police infiltrators. Right now, social media, with all the problems that we're dealing with, people disagreeing, some people have said, "Oh, some of these people that just always start arguments-maybe they're police infiltrators" (Katie, Occupy Los Angeles).

Showing the entanglement between their national Occupy network and state forces, Jane from OLA described how the police targeted their media tent at the precise moment of a pre-arranged, national 'inter-Occupy' Skype conference call:

The first night that we were set up to actually do one of the calls from our tent, a media tent at Occupy $L A$, the bomb squad showed up. They came right into 
that tent. They didn't really go anywhere else on the grounds [laughs]. And we're like, "You guys gotta go!" and I said to myself, we must be doing something really consequential if the bomb squad's gonna' try to coerce us out of this area, and this tent, and try to get us to leave. And of course we said that we weren't going and that they could blow us up. (laughing) and it would be fine and so $i$ was just sort of trying my best to get everything accomplished that $i$ thought would be able to set us up for something better once $i$ knew the raids were coming and the camps were gonna' be decimated (Jane, Occupy Los Angeles).

The 'coincidence' of a bomb-squad arriving to disrupt the national Occupy Movement call, saw the participant anticipate the police raids that would occur shortly thereafter: the internationally-orchestrated, violent and forcible state and police shutdown of Occupy sites across North America on Nov 17, 2010 (Wolf, 2012).

Another participant describes sousveillance in terms of using tools to connect across the U.S.:

I had been working with people at OWS and in Philly and Portland about putting together this inter-occupy and this conference called Service and we were doing conference calls using wifi technologies and our mobile phones from city hall grounds.

The added risk with operating in these online 'public spaces' is the exposure to surveillance technologies. As described by Mann (2004):

Surveillance ... as with profiling, often operates in secret, in the context of larger peer-anonymous communities, thus breeding mistrust, which itself breeds more surveillance, as a vicious cycle. Not to forget, of course, the lack of inverse visibility that can lead to corruption of politicians who use secrecy to hide theft of public monies, and the like, in a surveillance-only society.

Speaking directly to the friction between surveillance and sousveillance, Mann writes: 
Surveillance often embodies an hypocrisy, e.g. 'we can watch you but you're not allowed to watch us'. And since surveillance is the veillance of hypocrisy, we might ask 'what is sousveillance the veillance of? Integrity is the opposite of hypocrisy. So we might argue that sousveillance is the veillance of integrity. Also worth noting, sousveillance is often implemented by media that is controlled centrally, and thus subject to surveillance.

Our interviews with participants who experienced conflicts, dissonance, violence at the hands of police, media, or military, reflect descriptions of sousveillance that appears to be the 'new normal' of undersight from beneath. Challenging Lorde's assertion that the 'master's tools will never dismantle the master's house,' the use of such digital tools and platforms contributed the otherwise unreported or underreported police and state violence against the Occupy protesters and camps.

\section{Conclusion: Commitments to the Long Haul of Social Change}

The closure of the North American camps did not mark the end of the Occupy Movement. Since the internationally orchestrated, violent shutdown of all Occupy camps on November 17, 2011, the movement has continued-some retaining an association with the term 'occupy' and others not. These local and regional direct actions include: Occupy Sandy, a successful relief effort after Hurricane Sandy, coordinated through the Occupy network of that region; and, Occupy the Hood, one of hundreds of organised occupations and protests in support of families or people whose homes are threatened with foreclosure. Most Occupy developments are highly local and reflect a high rate of success in accomplishing targeted goals. Such actions and efforts are often focused in particular neighbourhoods within a city; these include, Occupy the Hood (Los Angeles), Occupy Bernal (San Fransisco), and Occupy the Farm (occupations of public spaces for urban gardening). The established web-based networks of Occupy have played a key role in mobilising local direct action.

Despite the fact that master's tools may change our relationship to politics and media for the rest of our lives, the risks and costs of using tools developed by corporate behemoths are of increasing concern not only to activists but to everyday users of the Internet; while we populate Facebook, Twitter, and Google with extensive personal, financial, political, demographic details, these are taken and used for corporate profit. More recently services like Ello and Diaspora offered alternatives to Facebook; however, those involved in social movements are more likely to use services that ensure a critical mass of social media users. 
The concept of the master's tools usefully acknowledges contradictions effectively built into the tools themselves: for example, the interests of those who produce and profit from the tool are built into the tools' uses, entangling the user within contradictory interests. But the conceptual tool doesn't intend to answer the question of whether these tools can or cannot dismantle the master's house: this conflict is ongoing and currently unfolding within a global context. Activists expressed divergent perspectives about these paradoxical entanglements. While some Occupy participants celebrated the subversive use of Facebook by social movements, other activists are deeply concerned about the increasing privatisation of the Internet and the need for technological alternatives of all kinds.

The uses of alternatives such as mesh networks were demonstrated during the recent four-month Hong Kong Occupy movement of 2014; news and blogs reported on the initial use of the 'FireChat' application to organise, coordinate, and communicate for the first weeks of the movement (Meyer, 2014). Contradicting some of the hype about FireChat use, Jason Li notes the following: 'During the Hong Kong protests, everyone I spoke to said that Facebook and WhatsApp were by far the most commonly used communication tools' (2015). In a similar vein, authors Ivan Sigal and Ellery Biddle of Global Voices emphasise the precipitating "affect" that frequently "sparks" a revolution, despite traditional news hype that too often heroifies a new technology as the movement catalyst (Sigal and Biddle, 2015). While the future of mesh networks and the value of FireChat is contested and remains in question as a solution (Sigal and Biddle, 2015), Meyer describes the tension between corporate owned infrastructures, software, and digital tools and activist visions and aims: 'many see mesh networking as a new, more promising kind of Internet. Mesh networks are more secure and resilient. They're not as easy to dominate. As such, they seem ideal for disaster and protest situations' (Meyer, 2014).

Despite debates about the precise role of social media in different contemporary uprisings and protests since 2011, the Occupy movement is historically unique in the fact that events are readily communicated to witnesses around the globe through 'just in time' communication technologies, through virtually synchronous and one-to-many communications. These forms of communication and mobilisation employed by Occupy, enabled by the masters' tools, represent the new media foundation of contemporary hybrid social movements. As we anticipate new developments within hybrid social movements, the catch-22 of the entanglements between humans and technologies alert us to remain cognisant of a guiding thesis from Donna Haraway's 'A Cyborg Manifesto': 'Irony is about contradictions that do not resolve into larger wholes, even dialectically, about the tension of holding incompatible things together because both or all are necessary and true' (1991: 149). 
Irony aptly captures the paradoxical entanglements of contemporary social movements struggling for economic justice while simultaneously engaging and supporting corporate owned tools and platforms. The irresolvable frictions echo the truism of radical democracy as an unfinished project. The activists we interviewed recognised this, and clearly expressed their 'long-haul' commitment to the action, philosophies, and vision that the Occupy Movement calls for and represents. Queering the binaries-one effect of intraaction and entanglements-is not an overnight affair; the required long-haul commitment to social justice and economic equality is an unpredictable journey into uncertain terrain.

\section{Biographical Notes}

Megan Boler is Professor of media and education at OISE/University of Toronto. Her books include Feeling Power: Emotions and Education (Routledge 1999); Democratic Dialogue in Education (Peter Lang 2004); Digital Media and Democracy: Tactics in Hard Times (MIT Press, 2008); and DIY Citizenship: Critical Making and Social Media (eds. Ratto and Boler, MIT Press, 2014). Her previous funded research 'Rethinking Media Democracy and Citizenship' examined the motivations of producers of web-based challenges to traditional news. Her web-based productions include the official study guide to the documentary The Corporation (dirs. Achbar and Abbott 2003), and the multimedia website Critical Media Literacy in Times of War.

Jennie Phillips is a Doctoral Fellow with the Citizen Lab, University of Toronto. Her research aims to identify a distributed approach to developing crisis resilience within virtual civil society networks. With an MA in Education Technology, she has experience in Emergency Management, international development, training and technology with the federal government, private, non-profit and her company ellips design + consulting.

\section{Notes}

[1] 'Hybrid social movement' refers to contemporary convergence of social and digital media as key communication tools for social movement organising, "hybrid" referencing the fact that social movements now rely significantly on both online and offline networks. 
For further discussion of the hybridity of on- and offline practices within social movements, see for example Boler 2008; Boler and Nitsou, 2014; Castells, 2012. 'This hybrid of cyberspace and urban space constitutes a third space that I call the space of autonomy' (Castells 2012; 222).

[2] For a discussion of weak ties see for example Gladwell (2010). See also Wolfsfeld et al (2013).

[3] Gaby and Caren (2012) highlight that activists and supporters like to use the tools they know; the platforms are designed on the basis of sharing and contribution, and capitalizing on them allows tapping into large, dense networks and engaging the 'movement sympathisers.'

[4] Other scholars highlight the potential of online networks to impact to offline results (Tremayne, 2013; Aday et al, 2010, Conover et al, 2013). They can be used to connect individuals for resource mobilization, collective framing, and help navigate the challenges of mass organization and coordination (Bennett and Segerberg, 2012; Theocharis, 2013; Hounsell, 2011; Theocharis, 2014).

[5] '\#OCCUPYWALLSTREET: A shift in revolutionary tactics' was the Adbusters article that called for the OWS movement. July 13, 2011 https://www.adbusters.org/blogs/adbustersblog/occupywallstreet.html

[6] Other research shows that in the initial stages of the movement, Twitter was a dominant platform for communication (Schneider, 2011; Stirland, 2011; Tremayne 2013). Analyses of tweets disseminated throughout the movement (Tremayne 2013; Conover, 2013; Theocharis, 2014, Thorson et al., 2013) reveal how Twitter was used to facilitate the rhetoric between the activists, mass media, organizations and the public and well as to sustain the momentum of the movement. More specifically it was used as a platform for the local level to communicate time-sensitive police and protest information, facilitate interstate communication regarding related to mass media and narrative frames, to manage critical issues faced by the movement.

[7] This essay is grounded in findings from a funded Canadian Social Science and Humanities Research Council three-year research project, 'Social Media in the Hands 
of Young Citizens', (PI Megan Boler, 2010-2014). The mixed-methods project engages interviews, participant-observer ethnography, analysis of technological and artistic creations, and discourse analysis of texts. In this essay, as we are drawing from 80 interviews conducted in 9 different cities (across the United States, Toronto, and Melbourne, Australia). We will refer to the international reach of this Movement as 'Occupy' rather than 'Occupy Wall Street' which will be used to refer specifically to the Occupy participants in New York City. The research included semi-structured interviews with 50 Occupy Toronto activists, and 30 longer semi-structured interviews with women activists from seven US Occupy sites (Oakland, Los Angeles, Santa Cruz, Santa Barbara, Santa Ana, Wall Street, San Francisco). The research teams also conducted an intensive year of discourse analysis of Occupy related websites, Facebook pages, twitter hashtags, indy-video, Ustream. These findings were analyzed in part with Atlas.ti qualitative data research software.

[8] The Spanish movement was also called 'Real Democracy NOW' or 15M ('15M' standing for May 15) that occupied public spaces in Madrid during the spring and summer of 2011. $15 \mathrm{M}$ in turn had been inspired by the events begun in Tahrir Square and other Middle Eastern uprisings that came to constitute the 'Arab Spring' of 2011. See Postill (2014).

[9] Occupy, as with other horizontally-structured movements, is sometimes defined as a 'countercultural movement' (concerned with changing consciousness and awareness) rather than as a bona fide 'social movement' defined by definitive policy-oriented or legislative goals. However Occupy is classified, its meme 'We are the 99\%,' and the related concept of the $1 \%$ owning the majority of the world's wealth, represents the Occupy Movement's radically successful intervention into popular and political conceptions and vocabulary, giving voice to the realities of the growing income gap experienced in all corners of the globe. Additionally, this intervention shifted traditional news discourse to use the term 'economic injustice' for the first time rather than 'class warfare'.

[10] Like many of the movements taking place around the globe, they operate through organizational modalities also known as Transnational Advocacy Networks or TANS. Spanning state borders, and containing one or more members that are either non-state agents or do not act on behalf of the state (Risse-Kappen, 1995), a TAN is a set of 'relevant actors working internationally on an issue, who are bound together by shared values, a common discourse, and a dense exchange of information and services' (Keck and Sikkink, 1998: 2; Mitchell and Boissevain, 1973: 23). TANs are distributed and decentralized, simultaneously operating in domestic and international spaces. The distributed and decentralized modality of a TAN overlaps with the 'horizontal' organizational structure of many contemporary social movements. 
[11] As well, some of these clips are shareable without being posted via a video-streaming site; users are increasingly able to share videos in a resolution and data byte size that facilitates sharing.

[12] Surveillance scholar Vian Bakir describes the complexities associated with the uptake of technology: 'The emergence of Web 2.0 combined with convergence cultures created a media environment that, for a while, was poorly understood, allowing challenges to strategic political communication from lay-people going about their everyday lives. As the democratization of media production has become more actualized in Web 2.0, and as convergence causes more 'linking up' between web-based participatory media and mainstream media, we enter a media environment of complexity, chaos and populismwhat McNair describes as a shift from a 'control paradigm' to a 'chaos paradigm,' and what others refer to as the 'YouTube effect' .... Media agenda-building now seems to be a more open field ....' (Bakir, 2010: 4).

[13] Connective labour (Boler et al 2014) extends Bennett and Segerberg's (2012) discussion of the 'logic of connective action', in which 'connective action' distinguishes contemporary networked activism from more traditional 'collective action' central to social movement theory. While collective action frames do have a place in social movement theory when considering organizational coordination and identity, connective action networks are 'typically far more individualized and technologically organized sets of processes that result in action without the requirement of collective identity framing the levels of organization resources required to respond effectively to opportunities' (Bennett and Segerberg 2012, 750).

\section{References}

Aday, Sean, Henry Farrell, Marc Lynch, John Sides, John Kelly and Ethan Zuckerman. 'Blogs and Bullets: New Media in Contentious Politics', Technical Report, U.S. Institute of Peace (2010).

Adbusters. 'A Shift in Revolutionary Tactics'- the article that called for the OWS movement', Adbusters (2012) https://www.adbusters.org/blogs/adbusters-blog/occupywallstreet.html

Bakir, Vian. Sousveillance, Media and Strategic Political Communication: Iraq, USA, UK. (London: Bloomsbury Publishing, 2010).

Ball, James, Julian Borger and Glenn Greenwald. 'Revealed: How US and UK Spy Agencies Defeat Internet Privacy and Security', The Guardian (2013) http://www.theguardian.com/ 
world/2013/sep/05/nsa-gchq-encryption-codes-security

Barad, Karen. 'Interview with Karen Barad', in Rick Dolphijn and Iris van der Tuin (eds). New Materialism: Interviews and Cartographies (2012a): 48-70.

Barad, Karen. 'Intra-actions [interview by Adam Kleinmann]', Mousse 34: An issue about dOCUMENTA 13 (2012b): 76-81.

Bennett, W. Lance. 'The Personalization of Politics Political Identity, Social Media, and Changing Patterns of Participation'. The Annals of the American Academy of Political and Social Science 644.1 (2012): 20-39.

Bennett, W. Lance, and Alexandra Segerberg. 'The Logic of Connective Action: Digital Media and the Personalization of Contentious Politics'. Information, Communication \& Society 15.5 (2012): 739-768.

Bennett, W. Lance and Segerberg, Alexandra.* The Logic of Connective Action: Digital Media and the Personalization of Contentious Politics* (Cambridge: Cambridge University Press, 2013).

Boler, Megan (ed.). Digital Media and Democracy: Tactics in Hard Times (Cambridge, Mass: MIT Press, 2008).

Boler, Megan and Christina Nitsou. 'Women Activists of Occupy Wall Street: Consciousness-Raising and Connective Action in Hybrid Social Movements', in Martha McCaughey (ed.). Cyberactivism on the Participatory Web (New York: Routledge, 2014).

Boler, Megan, Averie Macdonald, Christina Nitsou and Anne Harris. 'Connective Labor and Social Media: Women's Roles in the 'Leaderless' Occupy Movement', Convergence: The International Journal of Research into New Media Technologies 20 (2014): 438-460.

Chalmers, Johnson. Blowback 2nd Edition: The Costs and Consequences of American Empire (New York: Holt, 2004).

Conover, Michael D, Emilio Ferrara, Filippo Menczer and Alessandro Flammini. 'The Digital Evolution of Occupy Wall Street', PLoS ONE 8.5 (2013).

Deibert, Ronald. Black Code (Toronto: Signal, 2013).

Deibert, Ronald and Rafal Rohozinski. 'Tracking GhostNet: Investigating a Cyber Espionage Network', Information Warfare Monitor (2009): 1-52.

Gaby, Sarah and Neal Caren. 'Occupy online: How Cute Old Men and Malcolm X Recruited 400,000 US users to OWS on Facebook', ${ }^{*}$ Social Movement Studies* 11 (2012): 367-374.

Gladwell, Malcolm. 'Small Change: Why the Revolution will not be Tweeted', The New Yorker (2010), http://www.newyorker.com/magazine/2010/10/04/small-change-3

Gray, Chris Hables. Postmodern War: the New Politics of Conflict. (New York: Guilford: 1997). 
Gray, Chris Hables, and Ángel J. Gordo. 'Social Media in Conflict Comparing Military and Social-Movement Technocultures'. Cultural Politics 10.3 (2014): 251-261.

Greenwald, Glenn. 'UK detention of Reprieve Activist Consistent with NSA's View of Drone Opponents as 'Threats' and Adversaries', The Guardian, 25 September (2013), http://www. theguardian.com/commentisfree/2013/sep/25/nsa-uk-drone-opponents-threats

Greenwald, Glenn, Poltras, Laura and MacAskill, Ewen. 'NSA Shares Raw Intelligence including Americans' Data with Israel' The Guardian, 11 September (2013), http://www.theguardian.com/world/2013/sep/11/nsa-americans-personal-data-israel-documents

Haraway, Donna. 'A Cyborg Manifesto: Science, Technology, and Socialist-feminism in the Late Twentieth Century', in Simians, Cyborgs, and Women: The Reinvention of Nature (New York: Routledge 1991), 149-182.

Hounshell, Blake. 'The Revolution will be Tweeted', Foreign Policy (2011 June). http://www. foreignpolicy.com/articles/2011/06/20/the_revolution_will_be_tweeted

Kiley, Brendan. 'Political Convictions? Federal Prosecutors in Seattle Are Dragging Activists into Grand Juries, Citing Their Social Circles and Anarchist Reading Materials', In The Stranger (2012 August 8) http://www.thestranger.com/seattle/political-convictions/ content?oid=14397498

La Rue, Frank. 'Report of the Special Rapporteur on the Promotion and Protection of the Right to Freedom of Opinion and Expression, Frank La Rue', United Nations General Assembly: Human Rights Council (2013 April) http://www.ohchr.org/Documents/HRBodies/HRCouncil/RegularSession/Session23/A.HRC.23.40_EN.pdf

Langlois, Ganaele, Fenwick McKelvey, Greg Elmer and Kenneth Werbin. 'Mapping Commercial Web 2.0 Worlds: Towards a New Critical Ontogenesis', Fibreculture 14 (2009) http:// fourteen.fibreculturejournal.org/fcj-095-mapping-commercial-web-2-0-worlds-towards-anew-critical-ontogenesis/print/

Li, Jason. 'Fact checking the hype around mesh networks and FireChat'. January 11, 2015. http://www.88-bar.com/2015/01/fact-checking-the-hype-around-mesh-networks-and-firechat/

Lorde, Audre. 'The Master's Tools Will Never Dismantle the Master's House'. Sister Outsider: Essays and Speeches (1984): 110-114.

Mann, Steve, Jason Nolan, and Barry Wellman. 'Sousveillance: Inventing and Using Wearable Computing Devices for Data Collection in Surveillance Environments', Surveillance \& Society 1.3 (2001): 331-355.

Mann, Steve. "Sousveillance”: Inverse Surveillance in Multimedia Imaging', in* Proceedings of the 12th Annual ACM International Conference on Multimedia *(New York, NY: 2004): $620-627$.

Mann, Steve. 'Equiveillance: The Equilibrium between Sur-veillance and Sous-veillance.' Association of Computing Machinery'. (2005 May 12) http://wearcam.org/anonequity.htm 
Mann, Steve. 'Veillance and Reciprocal Transparency: Surveillance versus Sousveillance, AR Glass, Lifeglogging, and Wearable Computing',* IEEE ISTAS *(2013): 1-12.

Mann, Steve. 'Veillance: Beyond Surveillance, Dataveillance Uberveillance and the Social Implications of Microchip Implants', Emerging Technologies (2014): 32.

Masover, Steve. 'Not Your Granddaddy's Metadata: Don't Believe the PRISM Anti-Hype', Daily Kos (2013 June 7) http://www.dailykos.com/story/2013/06/07/1214589/-Not-yourgranddaddy-s-metadata-don-t-believe-the-PRISM-anti-hype\#

Meyer, Robinson. 'What Firechat's Success in Hong Kong Means for a Global Internet', The Atlantic (2014 October 6), http://www.theatlantic.com/technology/archive/2014/10/firechatthe-hong-kong-protest-tool-aims-to-connect-the-next-billion/381113/

Soderman, Braxton, Nicole Starosielski and Jussi Parikka. 'Circulating Concepts: Networks and Media Archaeology: Interview with Jussi Parikka', Amodern 2: Network Archaeology (2014) http://amodern.net/article/jussi-parikka-interview/

Pempek, Tiffany A., Yevdokiya A.Yermolayeva and Sandra L. Calvert 'College Students' Social Networking Experiences on Facebook', Journal of Applied Developmental Psychology 30 (2008): 227-238.

Pillay, Navi. 'Opening Remarks by Ms. Navi Pillay, United Nations High Commissioner for Human Rights to the Side, Event at the 24th session of the UN Human Rights Council How to Safeguard the Right to Privacy in the Digital Age?', United Nations Human Rights Council (2013 September), http://www.ohchr.org/EN/NewsEvents/Pages/DisplayNews. aspx?NewsID=13758\&LangID=E

Ratto, Matt and Megan Boler (eds). DIY Citizenship: Critical Making and Social Media. (Cambridge: MIT Press, 2014).

Scott, Susan V., and Wanda J. Orlikowski. 'Entanglements In Practice: Performing Anonymity Through Social Media', MIS Quarterly 38.3 (2014): 873-893.

Schneider, Nathan. 'From Occupy Wall Street to Occupy Everywhere', The Nation (2011), http://www.thenation.com/article/163924/occupy-wall-street-occupy-everywhere\#

Shea, Pip, Tanya Notley and Jean Burgess. Fibreculture 25 CFP (2014) http://fibreculturejournal.org/cfp-entanglements/

Shirky, Clay. 'The Political Power of Social Media Technology, the Public Sphere, and Political Change', Foreign Affairs (2011), http://www.foreignaffairs.com/articles/67038/clay-shirky/ the-political-power-of-social-media

Sigal, Ivan and Ellery Biddle. 'Our Enduring Confusion About the Power of Digital Tools in Protest', Fibreculture 25**(2015).

Stirland, Sarah Lai. 'Hacktivists Begin Operation Occupy Wall Street', Talking Points Memo (TPM) (2011), http://talkingpointsmemo.com/idealab/hacktivists-begin-operation-occupywall-street 
The Citizen Lab. 'Planet Blue Coat', Research Brief 13, Munk School of Global Affairs, University of Toronto, January (2013): 1-30.

Theocharis, Yannis, Will Lowe, Jan W van Deth and Gema García-Albacete. 'Using Twitter to Mobilize Protest Action: Online Mobilization Patterns and Action Repertoires in the Occupy Wall Street, Indignados, and Aganaktismenoi Movements', Information, Communication \& Society 18.2 (2014): 202-220.

Theocharis, Yannis. 'The Wealth of (Occupation) Networks? Communication Patterns and Information Distribution in a Twitter Protest Network', Journal of Information Technology and Politics 10.1 (2013): 35-56.

Thorsona, Kjerstin, Kevin Driscoll, Brian Ekdalec, Stephanie Edgerlyd, Liana Gamber Thompsone, Andrew Schrock, Lana Swartz, Emily K. Vragah, and Chris Wells. 'YouTube, Twitter and the Occupy Movement: Connecting Content and Circulation Practices', Information, Communication \& Society 16.3 (2013): 421-451.

Tremayne, Mark. 'Anatomy of Protest in the Digital Era: A Network Analysis of Twitter and Occupy Wall Street', Social Movement Studies: Journal of Social, Cultural and Political Protest 13.1 (2013): 110-126.

Wolf, Naomi. 'Revealed: How the FBI Coordinated the Crackdown on Occupy', The Guardian (2012 December 29), http://www.theguardian.com/commentisfree/2012/dec/29/fbi-coordinated-crackdown-occupy

Wolfsfeld, Gadi, Elad Segev and Tamir Sheafer. 'Social Media and the Arab Spring: Politics Comes First'. The International Journal of Press/Politics 18.2 (2013): 115-137.

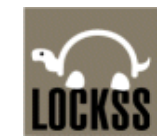

The LOCKSS System has the permission to collect, preserve and serve this open access Archival Unit

This Isuue of the Fibreculture Journal by The Fibreculture Journal Incorporated is licensed under a Creative Commons Attribution 4.0 International License.

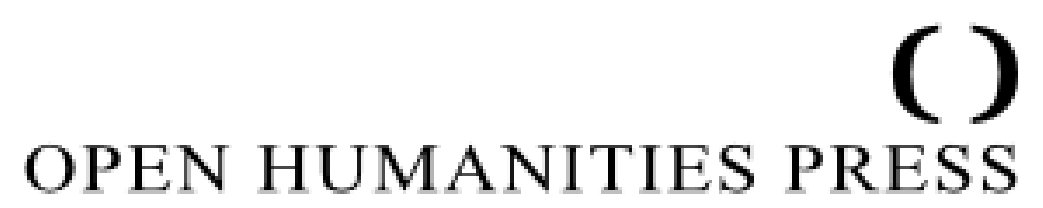

The Fibreculture Journal is published by The Fibreculture Journal Incorporated in partnership with Open Humanities Press. 


\title{
The Fibreculture Journal
}

DIGITAL MEDIA + NETWORKS + TRANSDISCIPLINARY CRITIQUE

\section{issue 26: Entanglements - Activism and technolog:}

\section{FCJMESH-005 Technology and Citizen Witnessing: Navigating the Friction Between Dual Desires for Visibility and Obscurity.}

\author{
Sam Gregory \\ WITNESS
}

\begin{abstract}
WITNESS has been training and supporting rights activists and citizen witnesses around the world to use video for over 20 years. Since this time they've trained people from 100 countries, supported successful advocacy campaigns and advocated for innovation in human rights technology. In this article, Program Director at WITNESS, Sam Gregory, discusses the events, reasons and philosophies that have informed their work and enabled them to support many people to use video and related technologies effectively, safely and ethically.
\end{abstract}

doi: 10.15307/fcj.mesh.005.2015

Put yourself in the shoes of an activist: perhaps a long-term Human Rights Defender or a firsttime citizen witness. You are filming on your cell phone in a repressive regime-capturing the testimony of someone assaulted by the police or documenting a protest.

After recording your video you are faced with choices about how your video should be used. You can upload your footage to a popular consumer platform like YouTube or Facebook. This option offers the capacity for rapid dissemination, which might help secure timely media and public attention; but these platforms also pose a risk, as they are readily accessible to the police and military. Because of this, the faces of the people you have filmed and the 
metadata describing the location and timing contained within the image might incriminate both you and them. Metadata captured by your mobile carrier and by the real-name-based social media service you have used, will likely add to this risk. This option means thinking through risk and the precautions you might take-like blurring faces and attempting to remain anonymous.

To avoid being incriminated or incriminating others, you might instead choose to hold onto your footage and to only share it with people you trust, as part of an investigation or even as potential evidence in a prosecution. In that case, it is important that you don't blur the faces of the people you have filmed, or obscure any vital authenticating information on location, timing or the chain of custody. This information will all become vital for your video to pass basic authentication tests [1].

Perhaps though you want to do both of these things: you might want to share information safely in the moment-so that it will quickly inform as many people as possible on what is happening-while also securing your information and metadata for later use and long-term accountability. This third option will see you challenged by the competing demands of obscurity and visibility, while negotiating consumer communications technologies that are not built for either of your needs, let alone for both.

These different choices illustrate one of the many frictions that emerge when we use contemporary consumer communication technologies in an expanding activist ecosystem that involves new participants, new approaches and new technologies: the friction between staying hidden and being found. This is a particular concern for those of us working in the area of human rights documentation since these services have not been designed with any awareness of the different needs and contexts of rights advocates and most often have in-built hidden surveillance and monitoring capabilities.

As one activist, 'Rafeeq' (2012) noted in the early days of the Syrian uprising:

Many of my friends were arrested for protesting. However they weren't arrested from the protest sites, but rather from the checkpoints spread across the city.

But how did Assad forces know they protested? Government forces have special teams 
dedicated to monitoring protests that we film and upload to the Internet. One of my friends who was detained for a short period told me that as he was undergoing torture in detention, he was asked by the investigator if he ever participated in rallies against the regime. When my friend denied protesting, the investigator showed him footage where his face clearly appeared in a protest.

This raises important questions about how much control creators, sharers, as well as people included in images, have over their identity when knowledge of their identity may bring real risks from perpetrators and state oppressors. These questions highlight the ways that the desire for immediacy in human rights reporting is compounded by expectations embedded in social media and in $24 / 7$ news reporting. Immediacy is usually critical for getting coverage of rights violations, while this content also needs to 'stand out' and be findable. Added to this, if this content is going to have legitimacy it must stand up to scrutiny by news analysts, NGOs and other justice institutions, courts as well as informed citizens.

This means, a citizen activist in Syria like 'Rafeeq' who shares a witnessing video must weigh up the risks of further exposing the identity of him or herself, allies, victims and testimony-sharers, with an awareness that their heart-breaking, powerful testimony to gross war crimes violations, may well get lost, ignored or mistrusted amidst the half a million plus videos and images of human rights violations that have emerged from that conflict over the past four years, let along lost amidst a much larger sea of non-human rights content.

When evaluating the risks and benefits of sharing human rights documentation of rights violations, media producers need to make assessments at multiple levels. Firstly, they need to consider what is or might be revealed by the pixels and audio recording; but secondly, they will need to consider the metadata that is attached to the recording. This underlying and often obscured data can tell us so much about the what, where, who and when. [2]

Each of these risk assessment levels is enabled or constrained by the tools that an individual uses, the media literacy they have in using them, and the policy scope and limits of the platform they use. Legal and extra-legal government surveillance applied the aggregate level-to both multiple users (through dragnet surveillance) and to individual users (through targeted surveillance)-add an additional layer of risk.

In our work at WITNESS, we have grappled with these levels of risk and opportunity in the 
course of advocating around the issues of 'visual anonymity' [3] and 'metadata for good' in our work in preparing training materials, designing technology tools (with our partners at the Guardian Project), and advocating to major consumer technology platforms. In our work with companies like YouTube we have used our own work designing activists tools with the Guardian Project such as ObscuraCam to provide reference designs for the introduction of new tools [4] that allow individuals to more easily blur faces, and we continue to advocate for tools to strip out metadata from within platforms. We have also actively argued for the converse: we advocate for 'eyewitness' and 'proof' modes and functionalities based on our InformaCam tool that enable the ability to add in metadata to enable video to be more easily found and trusted by news journalists and human rights advocates and to provide verification that it comes from the stated user and has not been tampered with (which is critical if video is to be used as evidence in court proceedings).

In advocating for these changes to the design and functionality of technology platforms we are challenging some underlying tenets of the current generation of popular Internet platforms. These platforms-including popular social network sites-have an emphasis on pushing for the use of 'real-name' identities and in preventing or resisting anonymity; this trend has been made manifest by Facebook's well-publicised battles with people who use pseudonyms. In this way, Silicon Valley's expectations around transparency and safety needs are very distant from the on-the-ground realities of vulnerable individuals in many parts of the world and in their own backyard; yet widely-used global consumer platforms like Facebook constantly push everyone to operate with the same norms of visibility and accountability. The emergence of popular platforms and tools from outside Silicon Valley may not necessarily change this, particularly when these tools and platforms emerge from authoritarian contexts like China, or countries with strong regimens of surveillance like South Korea.

Added to this, is the challenge of an emerging dominant cultural discourse that tends to frame metadata negatively. Metadata-the information such as geotags, timestamps, names, dates and locations that is associated with media we create-has been developing a bad name of late and has all but become synonymous in the popular imagination with government surveillance. In the post-Snowden moment, there is growing public distrust and disquiet about the use of "metadata for bad" not only by extra-legal government surveillance but also ranging from poachers using metadata in Instagram photos of wildife shot by tourists, to drone strikes authorised on the basis of patterns of mobile metadata, to women being stalked after the metadata contained in the images and video they have generated has revealed their location. 
At WITNESS, our response to these valid concerns about metadata is that much depends on how much control we have. Technologies, and the affordances they limit or support, can put control in the hands of the creator and sharer by incorporating a starting point of privacy-by-design and default and by allowing users to be as visible and discoverable as they want to be; or it can take away control and prevent us from blurring faces, stripping out metadata, and operating under a pseudonym. While allowing privacy-by-design and default, platforms can also allow individuals to choose to add in additional metadata and markers of trust that can help content to be found and be more likely to be trusted by people who are looking for real-time information in a sea of content.

At WITNESS we advocate that the frictions that emerge between technology, activists, anonymity and visibility can be better negotiated if all of us share some basic capacities and knowledge. In the expanding realm of human rights documentation, visual imagery is not just created by professional investigators or journalists, but by citizen witnesses and media activists as well as accidental, incidental and intentional witnesses. There is a resource allocation question around broadly building witnessing and documenting literacies and this raises the question: does everyone need to have a better understanding of how to make informed choices about the visibility and obscurity contained in the video and images we record and share?

We would argue yes. In an age where communication is increasingly visual and where citizen witnessing is used not just in human rights contexts but also for news and social media discussion, new capacities and skills are critical if we are to support the privacy rights of others and protect the most vulnerable from harm. But if citizens are to be capable and empowered technologically and personally to make the most appropriate choices about what they share and what they don't, we have to ground new citizen media literacies in the context of more responsible corporate practices and accessible, useable valuesmanifesting technologies that are part of the platforms that regular people utilise and not just in activist niches. And ultimately these mainstreamed technological choices and widespread literacies need proportional and human-rights respecting legal protections and sound public policy if citizens are to be capable, empowered (technologically and personally) and protected to make the best choices for themselves.

\section{Biographical Note}

Sam Gregory helps people use the power of the moving image and participatory 
technologies to create human rights change. He is Program Director at WITNESS, the leading organisation focused on empowering millions of people to use video for human rights effectively, safely and ethically, and he teaches on human rights and participatory media as an Adjunct Lecturer at the Harvard Kennedy School. He launched the Webbynominated Human Rights Channel on YouTube, and leads the WITNESS team working on the award-winning ObscuraCam and InformaCam tools. Sam has worked on impactful campaigns worldwide (particularly in South-East Asia and Latin America), and created innovative training programmes and teaching texts. Sam was a 2010 Rockefeller Foundation Bellagio Resident on the future of video-based advocacy, a 2012 Young Global Leader of the World Economic Forum, and in 2013 was named a 'Future for Good' Fellow at the Institute for the Future, working on 'co-presence for good' and live and immersive witnessing for human rights. Sam has a Masters in Public Policy from the Harvard Kennedy School, which he attended on a Kennedy Memorial Scholarship, and a BA First Class from the University of Oxford.

\section{Notes}

[1] For more information on the demands of evidentiary citizen media see 'Video As Evidence: Basic Practices', Kelly Matheson on WITNESS Blog, http://blog.witness. org/2015/02/video-as-evidence-basic-practices/

[2] For more on how these risks can play out see Sam Gregory and Elizabeth Losh. 'Remixing Human Rights: Rethinking civic expression, representation and personal security in online video', First Monday 17.8-6 (August 2012).

[3] For more on the concept of 'visual anonymity' see Sam Gregory. 'Human Rights Made Visible', in Meg McLagan and Yates McKee (eds) Sensible Politics: The Visual Culture of NonGovernmental Activism (Zone Books: 2012).

[4] For more information see 'Visual Anonymity and YouTube's New Blurring Tool', Sam Gregory on WITNESS blog, http://blog.witness.org/2012/07/ visual-anonymity-and-youtubes-new-blurring-tool/ 


\section{References}

Gregory, Sam. 'Human Rights Made Visible', in Meg McLagan and Yates McKee (eds) Sensible Politics: The Visual Culture of NonGovernmental Activism (Zone Books: 2012).

Gregory, Sam and Elizabeth Losh. 'Remixing Human Rights: Rethinking civic expression, representation and personal security in online video', First Monday 17.6-8 (August 2012).

'Rafeeq', 'The camera in Homs: a double-edged sword', Al Jazeera Blogs, published 28 June 2012, http://blogs.aljazeera.com/blog/under-siege-syrian-diary/camera-homs-doubleedged-sword

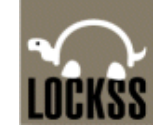

The LOCKSS System has the permission to collect, preserve and serve this open access Archival Unit

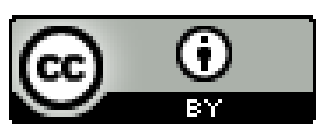

This Isuue of the Fibreculture Journal by The Fibreculture Journal Incorporated is licensed under a Creative Commons Attribution 4.0 International License.

\section{OPEN HUMANITIES PRESS}

The Fibreculture Journal is published by The Fibreculture Journal Incorporated in partnership with Open Humanities Press. 


\title{
The Fibreculture Journal
}

DIGITAL MEDIA + NETWORKS + TRANSDISCIPLINARY CRITIQUE

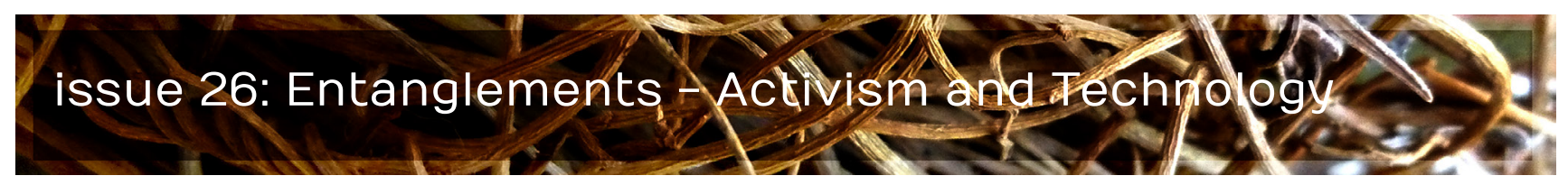

\section{FCJMESH-006 \\ From Information Activism to the Politics of Data.}

\author{
Maya Indira Ganesh and Stephanie Hankey \\ Tactical Technology Collective
}

\begin{abstract}
Tactical Tech has a decade of experience supporting the use of information and digital technologies to support rights activism. They say that in this time they have witnessed a radically altered information eco-system thanks to an explosion of new technologies, a dramatic rise in technology uptake and a burgeoning government and corporate surveillance system. Here Maya Ganesh and Stephanie Hankey, from Tactical Tech, discuss their analysis of the challenges this new information ecosystem poses for rights activism and they describe the ways in which Tactical Tech are choosing to address these challenges.
\end{abstract}

doi: $10.15307 /$ fcj.mesh.006.2015

As an organisation that supports activists and journalists to secure their communications to be more effective and more secure, we believe we have some parallels with groups working on a rather different issue: climate change. Environmentally friendly and sustainable lifestyle behaviours often start with having to recognise the easy short cuts and deeply ingrained habits we've gotten used to. Avoiding these short cuts and habits is something that many people invest a great deal of time and effort in when they do things like recycling, eat local produce or use a mobile app that tells them how to be a more ethical consumer. Climate change activists have developed a raft of inventive techniques to convey the message that 
many aspects of how we live are damaging the environment and that we should change these practices. At the same time, many people find this message challenging because they believe it seems too difficult to shift entrenched practices.

In our work at Tactical Tech we're trying to do something similar. We're raising awareness around day-to-day behaviours-specifically digital practices and technology choices. However, we're trying to do this at a time when digital networks and interfaces have never been more seductive: smoother, faster and far-reaching. With minimal effort we can share a video, voice our opinions, have flowers delivered half-way across the world, or access government data. The digital is as opaque as a magic trick; and the magic is in how the messiness of cables, trade, policies, digital workers, and politics are seemingly made to disappear behind a facade of frictionless sharing.

By asking what the flip side of frictionlessness sharing is, we are advocating for the opposite. We're trying to convey to activists that the excitement and ease of communication, self-expression and sharing enabled by the digital, often erodes freedom of expression, human rights, personal liberties and people's right to privacy. We ask the people we work with to rethink their digital habits and to consider more difficult choices. The thing is, very few people really want to do that.

\section{Then and Now}

Tactical Technology Collective started its work in 2003 to promote and build the capacity of NGOs, journalists, bloggers and activists to use free, libre, and open source software (FLOSS) and technologies in their activism and advocacy campaigns. At this time, the "Microsoft model" of computing was popular: the computer was a fixed device connected to the Internet with cables, where users paid companies such as Microsoft to run proprietary software. We also advocated for the use of digital tools in information activism: collecting and curating information to monitor corporations, powerful social institutions and state actors, holding them accountable while promoting local and global activism. Tactical Tech has also worked with activist communities in different countries to use digital tools to identify and evade targeted surveillance by governments threatened by freedom of speech. 
In the ten years since Tactical Tech began operating, Apple's market value has come to surpass Exxon Mobil's and the Internet is now heavily influenced by what we would term the "Google model": where every digital device and user has its facsimile in distributed data storage facilities (commonly referred to as "the cloud") [1]. Additionally, the Snowden leaks in 2013 revealed deep and pervasive collusion between corporations and governments to enable the surveillance of Internet users; we learnt that technology companies and the governments that regulate them, have developed an obsessive compulsion to collect and store data that most Internet users would deem personal or private. These conditions make the practice of and education about digital security, challenging. In an environment of mass surveillance, how is it possible to enable security and privacy measures if every digital act can be traced back to you, and with ever increasing levels of detail?

As a result of this environment, our work now encompasses the politics of data. Much like our early 'FLOSS-ophies' based on the merits of free, libre, and open source, the politics of data encompasses the technical, political and economic implications of the use of digital technologies. Our work in the area of supporting digital privacy rights is about encouraging awareness about risks and supporting activists to make different digital choices that resist, challenge or subvert the status quo; however, we're trying to do this at a time when the digital environment provides a host of ever-new and inspiring opportunities for activism.

\section{Data everywhere}

New media technologies are creating and collecting data about everything we say and do, which forces us to rethink how we present ourselves. There is information we know we're sharing about ourselves; there is information being generated about us that we do not create; and information about us is generated-unbeknown to us-simply because of our connections to other people online. These three kinds of information are being generated through digital activities and have been shown to compromise the security and privacy of activists, who have to rapidly develop new tactics of technology use in order to control their information flows.

In order to use the Internet now, different kinds of data are necessary to provide the services we've taken for granted: an identity, a location and a channel of communication. Whether using a real name or pseudonym, an email account will still be the key to a number of online services you may register for and therefore will provide a connection to your actions; this kind of chain of association means that eventually, you are always 
identifiable. Your location will also give you away: even if you never enable GPS on your digital device, using a service like Gmail or Facebook will give you a location that may not be physical-it may be in a network of people or based on where you travel online through linked services or even just your IP address that all these services log. Similarly, the channels we use to connect to the Internet, like ISPs, access points and service points, collect, store and relay information about users. This means that to completely avoid leaving behind data traces that can reveal our whereabouts, behaviours, and opinions, we need to consider, change or at least re-assess every single digital choice we make.

The kinds of personal behaviour changes we believe are required to minimise digital traces at Tactical Tech include choosing non-commercial or open source alternatives for everything from email to social networking, employing circumvention technologies and opting out of participatory practices associated with the 'sharing economy' [2]. However, we know that there are NSA and GCHQ programs designed to find and store information on users of the circumvention tool TOR and other privacy enhancing technologies. Even if we use tools designed to allow us to access or exchange information privately, we are rendered visible because of how out-of-the-ordinary our digital actions are. Also, many of these tools are difficult to use and install for those who don't have technical support.

The traces we leave and the way these can be used are extremely complex, particularly for activists. For example, during 'Operation Pillar of Defense'-the 2012 Israeli attack on Gaza-the well-known Egyptian activist Alaa Abd El Fattah tweeted certain views regarding Zionism. These tweets were later taken out of context and interpreted as 'calling for the murder of a critical number of Israelis.' This led to Abd El Fattah being stripped of the Sakharov prize in 2014, an award that acknowledges those who have defended human rights and freedom of thought. In a detailed response clarifying the context of the Twitter conversation, Abd El Fattah remarked, 'to pretend that you can interpret this tweet two years later without consulting the people involved in the conversation and to claim that it constitutes a call to action, is simply ridiculous.' Even as social media give us unprecedented reach and voice, what we do online can be accessed and used in ways beyond our control.

The struggle to control digital traces was also an issue for activists in the Hong Kong protests of late 2014. In this case the challenge was managing and making the right decisions about dual needs for anonymity and visibility. For those protestors who had been underground for many years prior to the street protests, it was important to remain undetected; however, the protests also provided an opportunity for these movements to become more active and potentially more effective. The choice to become actively 
involved risked complete exposure, partly due to how our digital traces never really disappear. Activists in Hong Kong were also targeted with spyware and had their websites attacked.

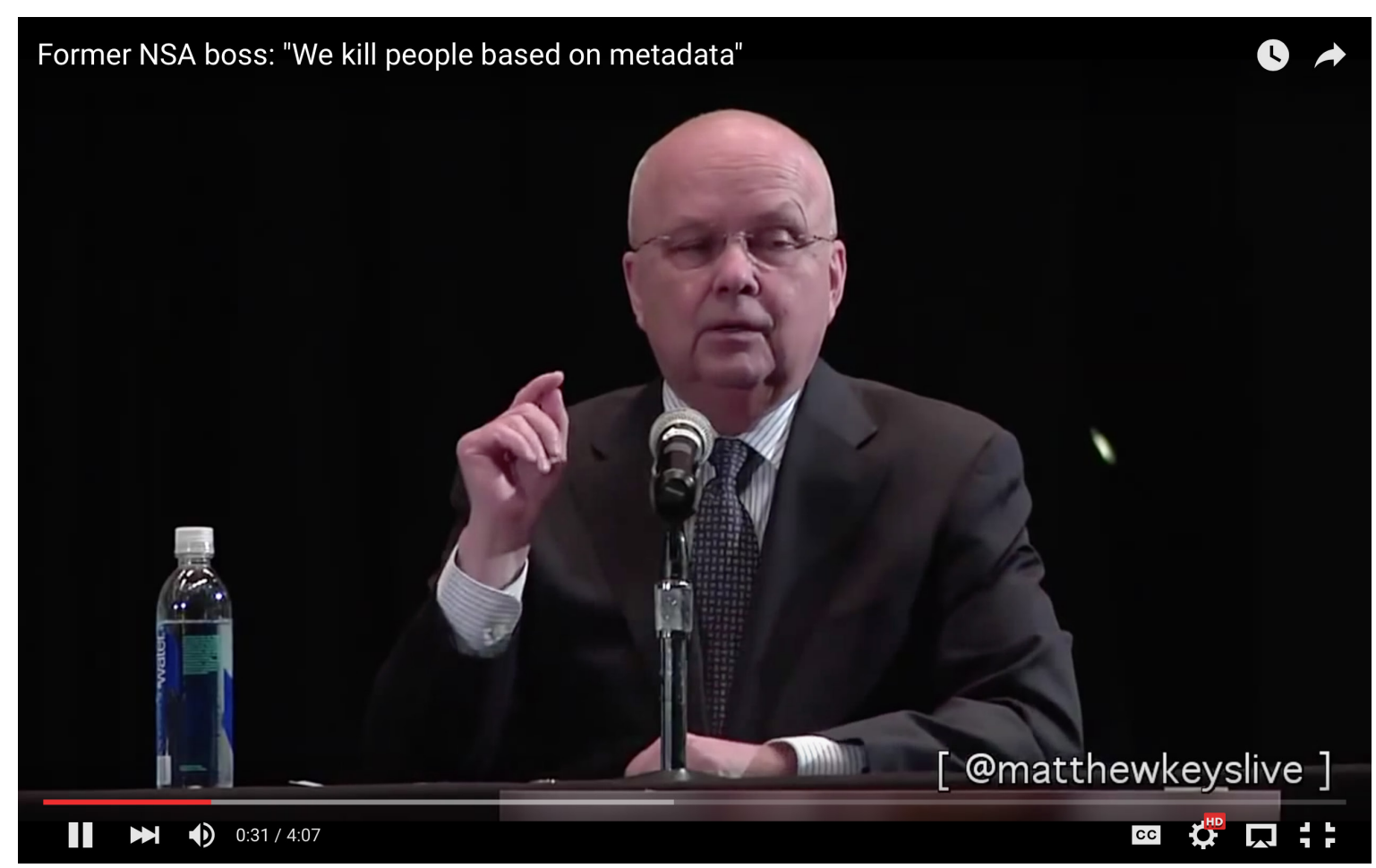

( $A$ youtube video is embedded in the online version of this paper - you can find that video at https://www.youtube.com/watch?v=UdQizOVavmc ) Figure 1. Haydn, Matthew. 'Former NSA boss: "We kill people based on metadata"', Youtube, published 11 May, 2014.

These examples help convey the complex information ecosystem activists now inhabit. In using online services and platforms in our work and everyday lives-whether we think about it or not-we are knowingly giving away data about our actions in exchange for free services and this has consequences and repercussions that may take years to unfold.

In many cases the data that leaves the messiest and least visible traces is metadata: information about all the information we create and consume. Metadata is an invisible layer of information on digital photos, in the logs of wifi access points-which also record all the other wifi points users have connected to over the previous 30 days - including call durations and times of phone calls. The power of metadata, often considered mundane and ubiquitous, became apparent when the former director of the NSA and CIA, Matthew 
Haydn (2014) remarked, 'we kill people based on metadata' during a dialogue about the practice of mass collection of communications information (see Figure 1).

Current debates about metadata often neglect to mention that it does not need to exist: that it is built into systems and products. News of end-to-end encryption of Whatsapp, the Facebook-owned instant messaging service, was received with enthusiasm for privacy adoption by commercial services; however, whilst the content remains obscure, the metadata associated with instant messaging - who messaged who, when, and how oftenis still seen by Whatsapp, and therefore by Facebook, and could still be accessible to government agencies with the capacity to read and analyse it.

Moxie Marlinspike, the founder of Open Whisper Systems that partnered with Facebook to encrypt Whatsapp says that the change is 'nearly invisible,' that '[o]rdinary users won't know the difference,' and that '[i]t's totally frictionless' (cited in Greenberg 2014). Marlinspike's evocation of frictionlessness emphasises the problematic idea that what is sold as efficiency and smoothness, ends up concealing how things really work. Integrating encryption into commercial software can be seen as a positive step, as it will help normalise the practice; however, a frictionless approach moves to obscure the complexities of privacy issues. Users may become complacent about the metadata attached to their encrypted messages and the companies who aggregate and analyse this information.

\section{Enabling awareness, alternatives, and action}

According to the security expert Bruce Schneier (2014), since Edward Snowden leaked information about the NSA's and GCHQ's mass surveillance programs, 700 million people worldwide have taken steps to be more private in their communications. Through our work at Tactical Tech we see a small slice of what this means for activists; an increasing demand for training, support and an increase in the number of people accessing our online digital security toolkit from 417,000 users in 2012 to 2.5 million by the end of 2014 .

One of the challenges in raising awareness about the new Internet we inhabit is that most users don't have the right metaphors or mental models to even comprehend its scale and velocity. Mental models affect how people choose to accept or reject information about the environment around them and their own role in influencing events. Design researcher, John Fass, is working on mental models of computing and draws on work by Evgeny 
Morozov who shows how hazy and misleading metaphors of how the Internet works are: the cloud, the Chinese 'fire-wall' and the cold war are thin metaphors that give misleading information about the material, political, and social realities of computing and Internet governance.

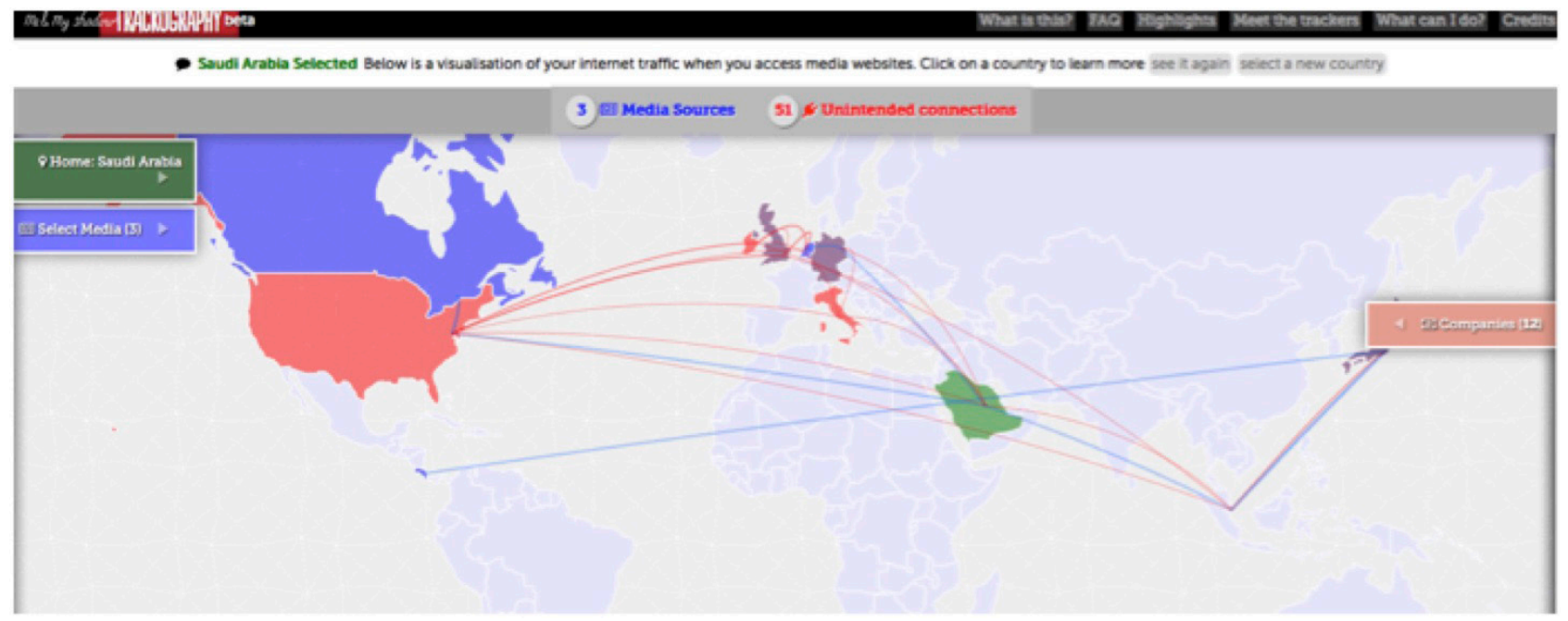

Figure 2: Screen grab of Tactical Tech's new project Trackography that reveals the third parties that have access to information about the news sites you visit online. Launched in December 2014, Trackography visualises data from news media websites and blogs in over 30 countries.

These metaphors are confusing and affect activists' ability to discard old behaviours and take on new digital practices. At Tactical Tech we have witnessed this in training workshops, from having to break the news that there really is no cloud in the sky that makes the Internet work, to making visible - in the form of dynamic visualisations-the data we give out from our devices as we move around a building, a city or a country. These mental models also compete with the slick narratives and metaphors about technology promoted by advertising and marketing of technology devices. Awareness raising and education also come up against some known arguments, like 'I have nothing to hide' or, 'I don't like it, but surveillance is required for national security.'

We try to take on these challenging, difficult arguments with strong evidence and convincing counter-arguments, or at least find the "sweet spots" that may serve to shift peoples' understanding of the problem. What is difficult about this is that we are saying to activists, in effect, that they have to limit, stop, or re-evaluate their own desires and practices online: that the biggest challenge is shifting their own behaviours. All we 
can really control are our own practices, and this is challenging as digital and mobile technologies have revolutionised how activists can use information in campaigning and in their personal lives.

\section{Learning from the Climate Change movement}

Advocating for and raising awareness about privacy and digital security for activists, is starting to mirror the climate change movement in certain ways. This involves developing scenarios about unseen, unknowable problems that deplete existing resources and erode peoples' power-in this case freedom of expression and democratic practices. We cannot conclusively prove these* *uture scenarios, but the abundance of evidence outlining current problems can be used to develop new methods to communicate to a broader audience.

The climate change movement has successfully encouraged individuals to take small steps to change their practices in favour of more sustainable ones. This has included actions like recycling and switching off the lights; in our scenario, we seem to be left with the individual acts of changing our privacy settings or not tagging friends in Facebook photos. However, it is clear in both the climate change and Internet freedom movements that these small steps, even in aggregate, are not going to make a dent in the system. Eventually, it is up to corporations and governments to make important new policy decisions and commitments. This is not likely to happen in any near future, however, as with climate change action, it takes people opting out, voting with their feet and choosing companies with better track records in order for viable alternatives and new markets to emerge; as with climate action, this can result in companies and governments feeling pressured to enact regulations, as opposed to talking about self regulation. For this reason, some of our new advocacy work is about using information technologies for transparency to reveal the workings of the data industries. Challenging the opacity of narratives, metaphors, and the workings of the data industries is one way to advocate for a change in digital habits and practices.

Still we are left asking: is it possible to inspire individuals to change their digital practices? We remain hopeful and concur with this statement made by investigative reporter Julia Angwin, author of the 2014 book Dragnet nation: a quest for privacy, security and freedom in a world of relentless surveillance [15]: 
We lived in a world where we were perfectly willing to tolerate our rivers catching fire and the air being filled with soot and people dying of black lung disease and then all of a sudden, after 50 years of that, we decided maybe we don't want that kind of world. And we've been very successful at cleaning up our environment. We did it partly through laws, but we also did it by changing our social norms. I think privacy is a similar social problem. It's something that we will change both through laws and also through being smart about what choices we make about what technology we use. (Julia Angwin 2014).

\section{Author Biographies}

Maya Indira Ganesh is Director of Applied Research at Tactical Technology Collective. She led the 'Evidence \& Action' Program 2011-2014 where she worked on writing the Visualising Information for Advocacy book and helped develop the Info-Activism Camp in 2013. Before joining Tactical Tech, Maya worked on one of the first studies of gender and online violence with APC's Women's Rights Program and on ICTs in development. Maya holds Masters degrees in Applied Psychology from Delhi University, India, and in Media and Cultural Studies from the University of Sussex, UK. She is persistent in her attempts to write consistently but is usually distracted by Twitter.

Stephanie Hankey previously worked with the Open Society Institute establishing their Technology Support for Civil Society Program, before co-founding Tactical Tech in 2003 and Tactical Studios in 2011. Stephanie has a background in information design, was editor-in-chief of Pulp magazine and worked as a creative director and producer for a number of London-based multimedia companies. She has a Masters in Information and Interaction Design from the Royal College of Art London and a certificate in Campaigning and Lobbying from NCVO. Stephanie is currently developing Tactical Tech's work on influence and visual persuasion and leading its new initiative on data shadows and political engagement. 


\section{Acknowledgement}

We would like to thank Marek Tuszynski and Bobby Soriano for their inputs.

\section{Notes}

[1] This is a business model that has profited a number of different technology companies; Google is just the biggest and most prominent of these. More details about NSA and GCHQ surveillance programs enabled by mass data collection can be seen here: https:// projects.propublica.org/nsa-grid/

[2] The sharing economy (sometimes also referred to as the peer-to-peer economy, mesh, collaborative economy, collaborative consumption) is a socio-economic system built around the sharing of human and physical resources. It includes the shared creation, production, distribution, trade and consumption of goods and services by different people and organisations. This definition is drawn from http://en.wikipedia.org/wiki/ Sharing_economy.

\section{References}

Angwin, Julia. Dragnet nation: a quest for privacy, security and freedom in a world of relentless surveillance (New York: Times Books, 2014).

Greenberg, Andy. 'Whatsapp Just Switched on End-to-End Encryption for Hundreds of Millions of Users', Wired Magazine online edition, published 18 November 2014, http://www. wired.com/2014/11/whatsapp-encrypted-messaging/

Haydn, Matthew. 'Former NSA boss: "We kill people based on metadata"', Youtube, published 11 May, 2014, https://www.youtube.com/watch?v=UdQizOVavmc

Schneier, Bruce. 'Over 700 Million People Taking Steps to Avoid NSA Surveillance', published 15 December 2014, https://www.schneier.com/blog/archives/2014/12/over_700_millio.html 
The LOCKSS System has the permission to collect, preserve and serve this open access Archival Unit

\section{OPEN HUMANITIES PRESS}

The Fibreculture Journal is published by The Fibreculture Journal Incorporated in partnership with Open Humanities Press. 
FCJMESH-006 From Information Activism to the Politics of Data 


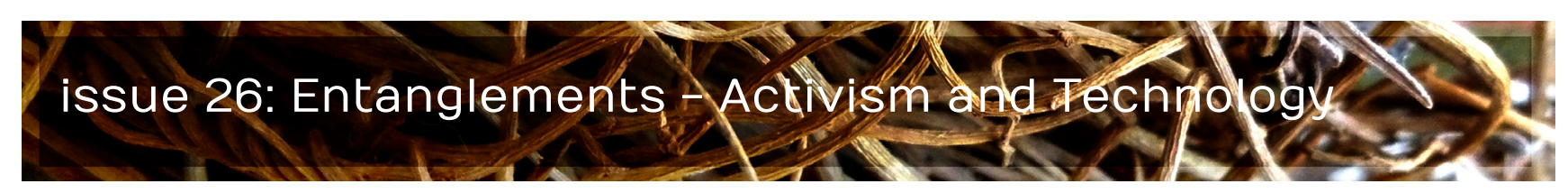

\title{
FCJMESH-007 Our Enduring Confusion About the Power of Digital Tools in Protest
}

\author{
Ivan Sigal and Ellery Biddle \\ Global Voices
}

\begin{abstract}
Since 2005 Global Voices has supported thousands of writers, online media experts and translators to share stories across borders and languages. Many of these stories have covered digital activism and protest around the world. In this article Ivan Sigal and Ellery Biddel share what they've learnt about protests, tipping points and technologies by reflecting on these stories and on the contrary narratives about these events that mainstream media have tended to focus on.
\end{abstract}

doi: 10.15307/fcj.mesh.007.2015

Did technology $X$ spark revolution $Y$ ? Pundits and political leaders have pointed to or blamed social media for driving the uprisings in the Arab region and for many other mass protests around the world, including those in the Philippines and Korea at the beginning of the century, as well as more recent uprisings in Burma, Moldova and Iran. In each case, it is clear that social media platforms have played a vital role in expanding the reach of communications and in helping like-minded activists to meet. It is equally clear that without the networks of activists who have worked together for years, laying the foundation for this groundswell, together with the convergences of many other social and political forces, each of these uprisings would either not have occurred or not have been sustained. 
At Global Voices, we cover stories that are not well represented in global media but are important to local communities. Our advocacy work focuses on online freedom of expression and governmental and corporate restrictions on the ability of individuals to speak, to listen, and access information. We also cover evolving stories of digital activism and protest around the world.

Over the past 10 years, the work of Global Voices has concentrated on eruptions of resistance to state and corporate authority within networked publics. Networked publics are communities of individuals for whom an overlay of telecommunications and Internet connectivity facilitates multiple, diverse links across time and space.

Since 2005 Global Voices authors have written more than 9,600 stories about protest, and nearly 10,500 stories about digital activism around the world. Additionally, we've created more than 60 special coverage sections that focus on digital activism, occurring in more than 40 countries. Many of the stories of digital activism and protest overlap, forming a body of material that gives us a rich picture of the relationship between digital technologies and their use in creating or supporting protest movements. These stories together form a set of data about emerging forms of protest and resistance, evolving tactics in the use of technology for protest and over time, a sense of the power dynamics that underlie these networks of individuals who coalesce around a range of political and social causes, many of which involve some form of assertion of rights.

Through this work we have found that groups of activists are aware of movements that have preceded them and are able to learn from their tactics, failures and successes in applying technology to their causes. Learning from other movements has led many activists to seek technology solutions that are both simple and widely available-cell phones, texts, email lists, cloud-based shared documents and popular social media platforms such as Facebook and Twitter, as well as older communication methods such as radio, pamphlets and word-of-mouth. And yet, the narrative of diverse communications choices rarely appears in mainstream media analyses; instead media coverage all too often emphasises new technology as the magic bullet that enables protests or mass uprisings. This misunderstanding shifts focus from the value of the protesters and the possible validity of their grievances onto a narrower story about the capabilities of new communications technologies.

After observing many protest movements over time at Global Voices, we can see a framework emerging that supports a richer understanding of the role that networked publics play in creating and supporting activism. We can see that protests will occur 
wherever networks of individuals are able to coalesce around a grievance and find symbols under which to organise. In 2011 we saw the Arab uprisings, crises protests in Europe and the Occupy movement; 2014 brought us protest movements in Ukraine, Venezuela, Taiwan, Mexico, and Hong Kong. This eruption of mass protests does not mean that the grievances were similar, or that the movements themselves necessarily had much in common in terms of strategy, goals or culture. Rather, the point is that the acceleration function of networks helps individuals find and learn from one another and to develop common causes.

Often utopian thinking espousing the power of technology obscures the stories that lie at the roots of a movement. Yet, observing dozens of protests emerge through reports at Global Voices we have seen something else happening. While we cannot predict the set of conditions which will lead a country to become the focus of future resistance and protest, we can recommend looking for the spark of resistance within networks of publics. Often, we have observed, that it is an event that occurs on the margins that serves as a catalyst, before becoming a symbol for a protest: often this symbol is human, charged with emotion and historically resonant.

The Tunisian revolution was lit by the match of the self-immolating Mohamed Bouazizi in the provincial town of Sidi Bouzid. Brazil's 2013 Vinegar Revolt was set off by a rise in public transport fees; but it was the vinegar that people used to endure tear gas that became the symbol of that movement. In short, the spark of protest is often an affect-shared and collective emotive responses to a piece of evidence about human consequences and emotions-rather than an effect, or a logical chain of reasoned argument. In this way we have learnt that it is the human, story-driven elements that bring people together to protest or create movements.

This is not to assert that technology is irrelevant or insignificant in recent mass protests and uprisings. Over the past several years we've seen the rise of mobile technology applications designed to serve activist ends, with affordances of anonymity, anti-surveillance, documentation and forensics, and management of evidentiary chains, such as the carefully considered applications created by the Guardian Project. Yet these tools can take years to develop and often serve narrow user groups of human rights activists, journalists and researchers. Their use in the context of mass protest requires either that enough protesters are aware of or learn to use the tools in time to be useful during events, or shortly thereafter. Some of these tools can be very effective, but many have never been widely adopted or effectively used in protest and advocacy contexts. 
Despite this, it seems as if with every protest or revolt a new technology receives glowing press coverage as the latest solution du jour. Often the technologies touted were not designed for protest environments and are not secure; yet the media's promotion of their value may drive further use. The overblown hype around the network encryption tool Haystack during the Iranian protests in 2009 may have been the apex of this misguided focus. Ironically, the lack of a technology's security combined with promotion of its use may create a hazard where none existed, as governments find new ways to surveil activist communities and where activists identify themselves through their use of these technologies.

Over time we see the emergence and repetition of a key tension: an ongoing mismatch between technologies and their application. On the one hand, we experience the often difficult and slow development of specialised tools that don't reach wide adoption. On the other hand, we endure reckless promotion of inappropriate and unsafe tools. Finally, this occurs amidst a misplaced emphasis on technology over the stories, symbols and concerns that spark and sustain activism.

In 2014-during the Umbrella Protests in Hong Kong-we saw a flurry of discussion about the use of the Bluetooth chatroom tool Firechat. As demonstrations peaked in early October, the Guardian newspaper's Archie Bland mused that '[e]very major display of social unrest these days seems to come with a game-changing technological accompaniment.' He went on to explain how Joshua Wong, the 18-year-old described as the "leader" of the Hong Kong protests had urged protesters to use FireChat, a chatroom app that is bluetooth-based and can thus be used when the mobile networks are overloaded or shut down. Bland went so far as to suggest that protesters may have identified with the infrastructural details of the app itself: 'The protesters may find something satisfying in the way the system works, gaining strength like a movement, or a radical idea, not through a top-down imposition, but from thousands of little connections (Bland, 2014).' According to Firechat, the app garnered nearly 100,000 downloads at the height of the protests. In the end very few people used the app, and despite fears to the contrary, mobile networks were never shut down.

While the bulk of the Global Voices coverage of the Hong Kong protests focused on the experiences of those who led the demonstrations, we felt the need to offer a critical view of FireChat. We were motivated by the amount of attention and interest it seemed to garner online-particularly in the digital activist community-but we knew the Hong Kong government was unlikely to shut down mobile networks. We also knew that the app's weak security standards could actually increase protesters' vulnerability to surveillance. We connected our contributors in Hong Kong with security experts familiar with the technology. 
Together they produced a story that identified the use of Firechat as a potential threat to protesters.

In Venezuela, the walkie-talkie app Zello was briefly in the spotlight in 2014. During protests in Caracas in February 2014, Zello reported more than 15,000 downloads in a single day. It was then promptly blocked, but only after President Madura announced that the government was monitoring protesters by intercepting communications sent using Zello.

These examples also show us that when commercial technologies are used to support mass protests, there's a power exchange between user and company. Products like Zello, FireChat, and Haystack benefit from the notoriety of being depicted as the tool of choice for protests. When they then reach out to protesters to encourage use, they are both asserting and bestowing power.

In these cases, and many others like them, we aim to dispel the notion that the success or failure of a protest movement hinges on the use of a particular technology. While it is easy to find articles about technology use and colourful umbrellas in mainstream western coverage of the Hong Kong protests, this focus downplays the fact that this movement is actually about people's demands for universal voting rights. Too often, the medium or the platform or the tool distracts mainstream media. In so doing, they lose sight of the message of the movement: the idea or grievance that's brought everyone together in the first place. In highlighting a single, highly touted tool or turnkey communications solution they obscure the fact that in protest we see that the use of diverse and often simple communications solutions provide movements with the resilient and reliable tools that serve their ends.

Beyond the debate about tools, we should refocus our attention on mass protests as events that form out of collective affect and produce symbols that represent the grievances and goals of the protesters. Too often, the human, story-driven elements that bring people to protest or create movements are not prioritised in media coverage. The long-term work of activists who have been fighting for their own and other's rights often struggle to 
identify and sustain those symbols and to turn them into real-world change. Technology can often serve them, but these activists are done a great disservice by the highlighting of technology over their realities, efforts, stories, and needs.

\section{Author Biographies}

Ivan Sigal is the executive director of Global Voices, a non-profit online global citizen media initiative and he supports similar projects around the world. He is a fellow at the Berkman Center for Internet \& Society at Harvard University, where he studies digital storytelling and online communities. He is a media producer who works on long-term photography, multi-media and writing projects. He is the author of White Road (Steidl, 2012), based on eight years of photography and writing about Central Asia and Russia. Ivan spent over ten years in the former Soviet Union and Asia, where he designed and ran dozens of media projects focusing on conflict, humanitarian disaster and transitional societies.

Ellery Roberts Biddle is the Advocacy editor of Global Voices where she facilitates a network of bloggers and advocates dedicated to reporting on threats to online speech, sharing tactics for defending the digital work and words of citizens and supporting efforts to strengthen Internet policy and practice worldwide. An expert on digital culture in Cuba, Ellery has worked as a policy analyst and human rights advocate in the digital rights field since 2010. Ellery is currently fellow at the Berkman Center for Internet \& Society at Harvard University, where she is continuing her writing and research on technology and the informal economy in Cuba.

\section{References}

Bland, Archie. 2014. 'FireChat - the messaging app that's powering the Hong Kong protests', The Guardian, Tuesday 30 September (2014), http://www.theguardian.com/ world/2014/sep/29/firechat-messaging-app-powering-hong-kong-protests 
The LOCKSS System has the permission to collect, preserve and serve this open access Archival Unit

This Isuue of the Fibreculture Journal by The Fibreculture Journal Incorporated is licensed under a Creative Commons Attribution 4.0 International License.

\section{OPEN HUMANITIES PRESS}

The Fibreculture Journal is published by The Fibreculture Journal Incorporated in partnership with Open Humanities Press. 
issue 26: Entanglements - Activis h and dechipology

\title{
FCJMESH-008 \\ Solutions for Online Harassment Don't Come Easily
}

\section{Jillian C. York Electronic Frontier Foundation}

\begin{abstract}
When popular technologies are being used to work against people, it is natural we look for solutions. But what if there is no perfect solution? In this article he Director for International Freedom of Expression at the Electronic Frontier Foundation, Jillian York, examines how social media harassment leads to complicated frictions between free speech and the protection of other basic human rights. ${ }^{*}$
\end{abstract}

doi: $10.15307 /$ fcj.mesh.008.2015

Don't feed the trolls.

Sticks and stones may break my bones, but names will never hurt me.

They're just words.

These are just a few of the things said to individuals-particularly women-who speak out about harassment they've experienced online. Time and time again, they are told to simply ignore it, to clamp down on their own privacy settings, or worse, that online harassment or stalking isn't real harassment or stalking.

The problem appears to be getting worse. As we increasingly spend large portions of our 
lives on social media platforms, it would be unwise to ignore the architecture of these services and the role they play in facilitating communication. The style of conversation enabled by a given platform can have unintended effects: for example, Twitter's anonymous, public, short-message format has been accused of fostering anger and harassment.

In light of this, discussions about harassment have come to the fore in recent months. Organisations such as the one I work for, the Electronic Frontier Foundation, have struggled to find the right answer in addressing this problem. While preserving free speech for all has traditionally meant fighting back against censors-be they governments or corporations-harassment itself can and does act as a censor, forcing its victims offline out of fear or exhaustion.

In an October 2014 article in the Atlantic, authors Catherine Buni and Soraya Chemaly argued that, by allowing certain offensive content to remain available on social media in the name of free speech, social media companies are effectively aiding in the silencing of women. In this same article, the following citations were made from a piece I had earlier written on the same subject: 'The question is not can Facebook censor speech, but rather, should it?' given that any censorship of content 'sets a dangerous precedent for special interest groups looking to bring their pet issue to the attention of Facebook's censors.' The authors of the piece criticised my use of the term 'pet issue' to describe a problem that affects half the world's population. In fact, I was not describing violence against women as a pet issue, but rather the Pandora's Box that has opened in response to allowing certain groups to assist companies in the policing of speech.

Twitter recently invited the Women, Action, and the Media (WAM!) network to assist the company to more effectively monitor and parse through reports of harassmentpresumably to help define what constitutes harassment within the platform. Following their initiation to the process and further media attention toward the harassment problem, Twitter convened several meetings with advocacy groups. Among the groups invited were EFF, as well as the Anti-Defamation League (ADL), an organisation with the stated goal of 'combating hate.' In practice, however, the ADL has long been implicated in attempts at censoring critics of Israel, particularly on college campuses.

The concern I share with colleagues at EFF is not that eliminating images encouraging rape will result in a slippery slope; rather, that special interest groups will persuade Twitter to do their bidding, resulting in the platform looking more like Facebook-which seeks to create a 'family-friendly space' by censoring heavily-than 'the free speech wing of the free speech party,' as Twitter's general manger once billed the site (quoted in: Halliday 2012). 
The plan set forth by Twitter and WAM! will undoubtedly work in the short term, as Twitter removes accounts set up to harass individuals; but the ease with which one can set up a new account on the platform means that dedicated harassers won't be gone for long.

Other solutions proposed thus far have focused less on treating the cause rather than the symptoms. There have been proposals to eliminate anonymity from the Internet, proposals to proactively censor certain words on social media platforms and proposals to prosecute harassers more harshly. There have been countless calls for social media companies to 'do something!' without much clarity into what 'something' might be. A problem with many of these solutions is that, in addition to potentially curbing harassment, they will surely have a chilling effect on other speech. For example, eliminating anonymity to stop harassment would also eliminate anonymity for those who need it most: such as, human rights defenders, victims of gendered violence, or young people.

The more prudent solutions have revolved around providing tools that give individuals more tools to report and blocking individuals. One such tool is BlockTogether, which gives individuals more control over who can follow or interact with them. BlockTogether allows users to create group blocklists that can be shared with others. Twitter is making its own dashboard easier to use, making the process of blocking a bit easier. These solutions allow users to feel safer without risking censorship, but may not go far enough in many cases.

These tools are not without their critics, who argue that mass blocking could have adverse effects if, for example, an individual were mistakenly placed on a block list that was then shared widely.

It seems, then, that there isn't a solution that will both ensure the protection of free speech while also ensuring that all individuals feel comfortable expressing themselves. And, in order to accomplish the latter, it seems increasingly likely that we will have to compromise on the former.

Harassment must never be tolerated, but suppressing it-rather than countering it-will not swiftly rid us of the problem, and is likely to create new ones. Concerns about who regulates speech are valid in a time when our major platforms for expression are designed and controlled by U.S. corporations governed by men. The agendas of carceral states must also be a concern, so that the pursuits of harsh, punitive remedies are not encouraged. Online harassment is a complex problem with no clear solutions, and this should be our starting point as we seek to address it. 


\section{Author Biography}

Jillian C. York is a writer and activist focused on the intersection of technology and policy. She currently serves as Director for International Freedom of Expression at the Electronic Frontier Foundation and is a Fellow at the Centre for Internet \& Human Rights in Berlin.

\section{Acknowledgement}

l'd like to thank Soraya Chemaly and Jaclyn Friedman for making me think harder about this problem, and my colleagues at EFF for being supportive in that process.

\section{References}

Buni, Catherine and Soraya Chemaly. 'The Unsafety Net: How Social Media Turned Against Women,' The Atlantic, 9 October, (2014) http://www.theatlantic.com/technology/archive/2014/10/the-unsafety-net-how-social-media-turned-against-women/381261/

Halliday, Josh. 'Twitter's Tony Wang: 'We are the free speech wing of the free speech party', The Guardian, 23 March, (2012) http://www.theguardian.com/media/2012/mar/22/ twitter-tony-wang-free-speech

Nussbaum, Martha C. 'Haterz Gonna Hate?' The Nation 5 November, (2014) http://www. thenation.com/article/haterz-gonna-hate/

Owens, Simon. 'The Real Reasons Twitter Can Be So Brutally, Screamingly Terrible' nymag 22 October, (2014) http://nymag.com/scienceofus/2014/10/real-reason-twitter-can-be-horribly-terrible.html\#

York, Jillian C. 'Harassment Hurts Us All. So Does Censorship' Medium September 13, (2013) https://medium.com/@jilliancyork/harassment-hurts-us-all-so-does-censorship$6 \mathrm{e} 1 \mathrm{babd61a9b}$ 


\section{OPEN HUMANITIES PRESS}

The Fibreculture Journal is published by The Fibreculture Journal Incorporated in partnership with Open Humanities Press. 


\title{
The Fibreculture Journal
}

DIGITAL MEDIA + NETWORKS + TRANSDISCIPLINARY CRITIQUE

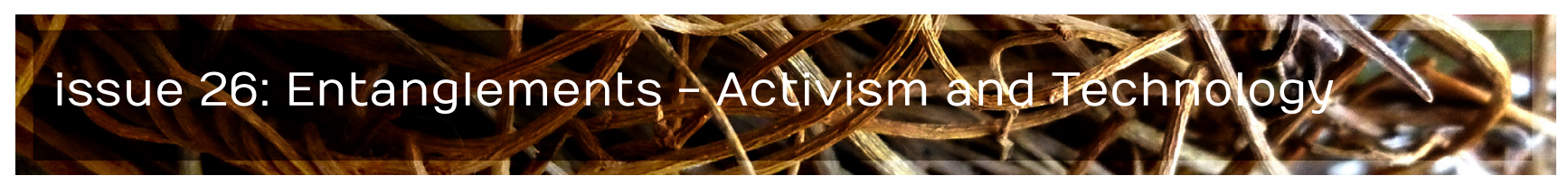

\section{FCJMESH-009 Ranking Digital Rights: Keeping the Internet Safe for Advocacy}

\author{
Nathalie Maréchal \\ Ranking Digital Rights Project
}

\begin{abstract}
The Ranking Digital Rights project is creating a system to evaluate the world's Internet and mobile companies on policies and practices related to free expression and privacy in the context of international human rights law. In this article, project researcher, Nathalie Maréchal, talks about the ideas and events that have informed the project and the challenges and opportunities involved in taking it forward.
\end{abstract}

doi: $10.15307 /$ fcj.mesh.009.2015

The techno-utopianism of the early 2000s has given way to new discourses warning about the threats that the Internet poses to democracy and human rights (Deibert, 2013; MacKinnon, 2012; Morozov, 2011). These discourses are now being shaped by Edward Snowden's revelations about the United States government's mass surveillance programs which have brought privacy issues to the fore, and by the subsequent proliferation of news reports about Internet safety and security which have reinforced that this is a serious issue that is not going away. Notwithstanding the United States government's protestations to the contrary, a recent report led the United Nations High Commissioner, Navi Pillay (2014), to conclude that mass surveillance-as opposed to targeted surveillance- amounts to a systematic interference with the right to respect for the privacy of communications. 
The rights to information, privacy and to free expression are among the fundamental rights included in the Universal Declaration of Human Rights. As human activity is increasingly mediated by the Internet, these rights are becoming prerequisites for enjoying all the other rights. Without access to information, how can citizens find out about problems in their communities? Without freedom of expression, how can they express their desire for change or debate the issues of the day? And without privacy, how can they strategise and coordinate advocacy efforts without being stymied by government or corporate actors?

In the United States where I live, work, and study, I have the ability to access information about violations of human rights, to express my views about them and to coordinate my research and advocacy efforts with colleagues around the globe. But this is far from a universal experience. In Thailand, lèse-majesté laws are so strict that anyone who publicly criticises the king or royal family risks a prison sentence. In China, the state's control over information is such that Mainland Chinese media was initially devoid of any mention of the pro-democracy protests in Hong Kong. (They eventually covered the movement from a very negative slant). The Hungarian government proposed a per-gigabyte tax on Internet downloads in 2014, which would have made many forms of online communication prohibitively expensive. There are also signs that many liberal democracies are trending toward increased communication control and repression, as the Snowden revelations illustrate. Canada's new Anti-Terrorism Act, Spain's Ley Mordaza and France's efforts to regulate online speech after the Charlie Hebdo terrorist attacks provide three worrying examples of the decline of civil liberties in the West.

The case of LGBT rights in Russia exemplifies the centrality of free expression and privacy for rights activism. Since 2013 it has been illegal to promote "non-traditional sexual relations" to minors, resulting in a de facto criminalisation of LGBT rights advocacy. This, combined with stringent Internet laws and Putin's 'information security doctrine,'which situates the control of information as a national security interest-has contributed to an increase in discrimination and assaults against LGBT Russians. Impunity prevails since hate crimes do not get reported, much less explicitly tagged as such. The Mayor of Sochi's infamous claim in the run-up to the 2014 Olympics, that 'there are no gays in Sochi,' was illustrative of the enforced obscurity endured by LGBT Russians. When the gay community's existence is denied, the idea of gay rights becomes inconceivable. Even among the minority who support LGBT rights, the climate of intimidation and fear has produced a chilling effect where would-be activists refrain from actions that might lead to reprisals, even when their activities may not be deemed illegal.

These, and other state-led assaults on privacy and freedom of expression, are documented in numerous rankings and reports published by the non-profit sector, including groups like Freedom House, Reporters without Borders, and others. But these reports usually neglect 
to examine the impact or role for Internet intermediaries like ISPs, mobile phone operators, social networking sites, and manufacturers of devices and networking equipment in carrying out-or preventing-oppressive policies. In the early 2000s, United States Internet giants Microsoft, Google and Yahoo! were harshly criticised-and rightly so-for cooperating with requests from the Chinese government to hand over user data, which resulted in at least two human rights defenders spending years in prison. In the wake of these events and ongoing criticism, the three companies formed the Global Network Initiative (GNI) in 2008 as a multi-stakeholder forum for dialogue with technologists, activists and other experts on best practices for balancing their business interests with human rights concerns in widely disparate social and legal contexts.

The GNI now includes six member companies, including Facebook, that hold one another accountable for respecting privacy rights and freedom of expression wherever they operate. While human rights have traditionally been considered the exclusive responsibility of the State, the human rights movement has gained traction by working with, rather than against, the private sector, and by making the business case that rightsrespecting practices produce a better rate of return on investment. Various industries (such as diamond extraction and manufacturing) have joined with academics and nonprofit organisations to form multi-stakeholder agreements or structures (for example the Kimberley Process to address diamond mining and conflict or the Fair Labor Association in the case of supporting worker rights) that promote adherence to international laws and norms. These groups do so through a variety of mechanisms, including certified audits and providing a confidential forum for companies to seek and receive advice on how to improve their operations' respect for human rights.

However, initiatives of this type (including the GNI) only have influence over their member companies, who are already committed in principle to respecting human rights. Rebecca MacKinnon, who helped start the GNI, started the Ranking Digital Rights (RDR) project as a way to hold the world's ICT companies accountable for respecting privacy and free expression, regardless of GNI membership. Though very different in methodology, the GNI and RDR are both based on the UN Guiding Principles on Business and Human Rights. These Guiding Principles, approved in 2011 after decades of fits and starts, represent a departure from 20th century international human rights law in that they assert that private sector companies, and not just governments, have a responsibility to respect human rights.

The Ranking Digital Rights (RDR) project is developing a system to publicly assess how well the world's information and communication technology (ICT) companies are living up to this responsibility vis-à-vis privacy and free expression rights. The process of drafting the ranking methodology has been deliberate and consultative, starting with a series of stakeholder consultations, geographically-focused case studies, two open-comment 
periods on the methodology itself and a [pilot study] focusing on 12 companies representing a range of markets, sub-sectors and legal environments.

The RDR indicators used to assess companies consider company commitments, policies and practices regarding human rights, freedom of expression and privacy. Each company's score is based on publicly available information, which we hope will incentivise firms to become more transparent. (The full methodology is available on the project website.) Sample questions we are using to develop indicators include:

- Does the company regularly conduct human rights impact assessments (HRIA) addressing how the company's products and services affect the freedom of expression and privacy of its users?

- Does the company provide evidence that it supports implementation by staff at all levels and throughout the company of its freedom of expression commitments?

- Does the company publish data at regular intervals about government requests it receives to remove, filter, or restrict access to content, plus data about the extent to which the company complies with such requests, if permissible under law?

- Does the company regularly conduct credible and independent security audits on its technologies and practices affecting user data?

In October 2014, we started a pilot study that examined and ranked 12 publicly traded ICT firms around the world, in preparation for the launch of a full annual ranking of several dozen of the world's largest publicly traded Information and Communication Technology (ICT) companies in late 2015. A public report summarising the pilot study was released in March 2015, although the scores for the pilot companies were not published since at this stage the methodology was still considered experimental and required further revisions before being applied publicly. Starting in 2016, the ranking will include hardware and networking equipment manufacturers alongside Internet Service Providers (ISPs), telecom operators, social networking sites, and other Internet companies.

So what good will this company ranking accomplish? The Ranking Digital Rights theory of change is complex (see Figure 1); however, the goal is to get companies to compete against each other on privacy and free expression metrics by giving consumers and investors accurate, comparable information by which to judge ICT firms. The information will also be crucial to local activist efforts to engage with companies and governments to ameliorate laws, policies and practices that contravene privacy and free expression. The 


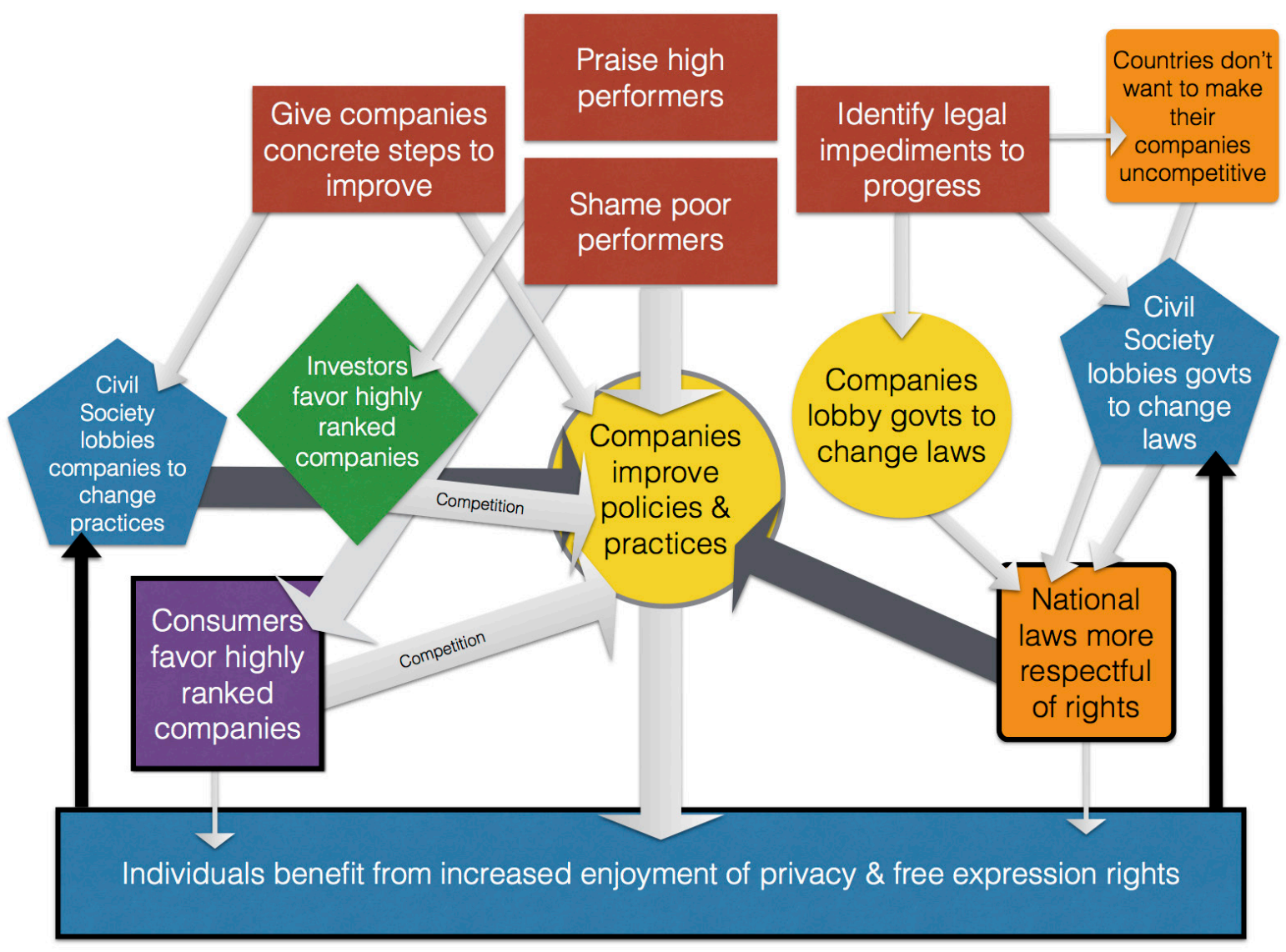

Figure 1: Ranking Digital Rights process map

end game is to create a virtuous cycle whereby baseline privacy and the capacity to freely express opinions makes the full spectrum of human rights more accessible.

The friction here, however, is between the early idea of the Internet as an alternative space free from the constraints of the physical world and the role played by ICT companies-as gatekeepers to the global public sphere-in implementing government-driven information controls. Ranking Digital Rights and related activist projects aim to highlight this friction by shining a spotlight on companies' performances and by empowering consumers as well as investors to decide which companies to favour.

There are also significant frictions between the worldviews of the rights advocacy and ICT sectors. Those within private sector companies may lack awareness of the impact of their products and services on the human rights of their users. Indeed, several company 
representatives we spoke to during 2013 were initially confused by the connection between their business operations and the concept of human rights. Similarly, a chasm remains between the mainstream Corporate Social Responsibility (CSR) world and those who advocate for digital free expression and privacy. Participants in a workshop we held in 2012 noted that mainstream CSR human rights related initiatives focus on concerns commonly associated with sectors like manufacturing and extractives which has little in common with their own work. Conversely, many Internet privacy advocacy groups and initiatives are disconnected from mainstream CSR approaches and in some cases feel more comfortable casting stones from an adversarial stance rather than engaging with companies. In our consultations with representatives from the ICT sector, we found that once the connection between CSR, human rights, and the ICT sector was spelled out-and the term "human rights" was rephrased as "user rights"-our interlocutors became more receptive to the project's goals. Part of the friction, at least, lay in the different semiotic worlds inhabited by the various stakeholders.

Perhaps the most important source of friction though, stems from the perception that human rights, accountability and transparency are Western concepts that can be dismissed in the name of national sovereignty or cultural diversity. Despite this, a growing number of NGOs outside of North America and Western Europe now exist with the primary purpose of promoting information rights. Many of these groups have expressed interest in using data from the RDR project as an advocacy tool at both national and regional levels. Also, a number of groups in a range of countries are currently devising schemes to rank the ICT firms in their own countries against each other, according to metrics grounded in domestic laws and concerns. This is a welcome development in many respects: firstly, more companies will be scrutinised than under the Ranking Digital Rights scheme; secondly, companies will be ranked against their direct competitors; thirdly, local activists are the real experts on the concrete needs and issues in their own countries and on the best combination of tactics to influence local actors; and finally, the existence of grassroots initiatives undermines claims that the digital rights movement is a Western aberration.

\section{Author Biography}

Nathalie Maréchal is a doctoral student in Communication at the University of Southern California Annenberg School for Communication and Journalism. She was the 2014 COMPASS Fellow at the New America Foundation, where she contributed to the Ranking 
Digital Rights Project.

\section{References}

Deibert, Ronald J. Black code: Inside the battle for cyberspace (Toronto: Signal, 2013).

MacKinnon, Rebecca. Consent of the Networked: The World-wide Struggle for Internet Freedom (New York: Basic, 2012).

Morozov, Evgeny. The Net Delusion: How Not to Liberate the World (London: Allen Lane, 2011).

Navi Pillay. 'Dangerous practice of digital mass surveillance must be subject to independent checks and balances', website of the Office of the High Commissioner for Human Rights, (2014) http://www.ohchr.org/EN/NewsEvents/Pages/DisplayNews.

aspx?NewsID=14875\&LangID=E

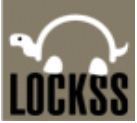

The LOCKSS System has the permission to collect, preserve and serve this open access Archival Unit

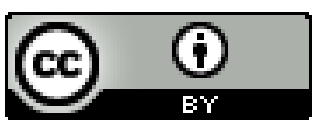

This Isuue of the Fibreculture Journal by The Fibreculture Journal Incorporated is licensed under a Creative Commons Attribution 4.0 International License.

\section{OPEN HUMANITIES PRESS}

The Fibreculture Journal is published by The Fibreculture Journal Incorporated in partnership with Open Humanities Press. 


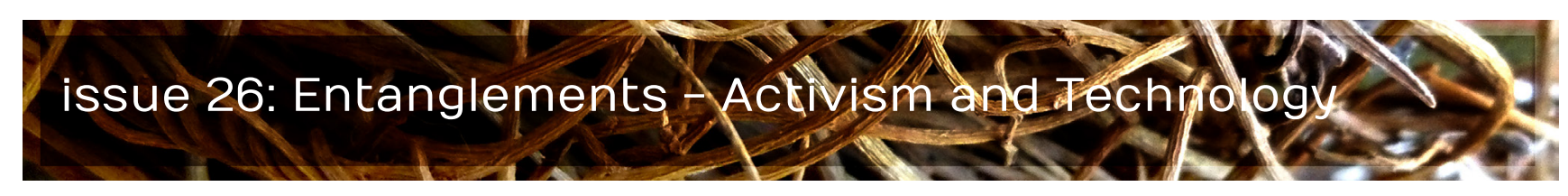

\title{
FCJMESH-010 Getting Open Development Right
}

\author{
Zara Rahman \\ the engine room
}

In this article Zara Rahman, from the engine room, explores tensions between the activist motivations that are driving the use of technologies in Open Development and the rights and aspirations of vulnerable people and communities. She discusses how we might and why we must resist the push for new technologies to be adopted too quickly in pursuing an Open Development agenda.

This article will look at a few issues around the Open Development movement, which is a relatively new agenda within the field of international development. Open Development is centred on the idea that 'openness'-in different formscan improve the international development sector.

doi: $10.15307 /$ fcj.mesh.010.2015

Generally, there are a few broad themes that come up in most definitions of what Open Development includes:

- the availability and use of (open) data relating to development programs

- participatory decision making and involvement from those affected by the program (a.k.a the "beneficiaries")

- innovative uses of technology to address development issues 
As the Open Development movement is reasonably new, different actors are interpreting the term in different ways. Also, as noted by Smith and Elder (2010), it is important to remember, 'openness is not one end of a binary dichotomy' and to understand it as a spectrum or range.

In this article I focus primarily on issues relating to open data, technology and accountability in the sector, because my experience lies in this realm; there are however, many other issues that could also be discussed to explore the complexities and frictions of open development as an ideology and a practice.

To consider the frictions that are emerging, let's start by thinking about the environment in which open development finds itself. By nature, international development projects involve some of the world's most vulnerable people, some of whom are living in the most difficult situations imaginable. Many are living in poverty, lack access to vital services, or have their basic human rights violated on a regular basis.

As such, the ethical and moral responsibilities for those working in the development sector are (or should be) even more demanding than in other sectors. The restricted agency of beneficiaries also means that the power balance within human rights, humanitarian and development projects are skewed towards the development practitioner. Reducing this power balance is difficult, if not impossible; being aware of it, however, is essential.

In many ways, the open development movement is (or should be) as much about critiquing the effect of new technologies and methods upon the societies in which we work, as much as it is about designing and implementing them. However, in practice, the immediate benefits of these technologies-as well as wider environment we find ourselves inwhere admitting failure is not looked kindly upon-is much more visible than the potential dangers or failures.

\section{Open data: transparency and accountability for who?}

The advent of open data-data which falls under the Open Definition-has played a large part in establishing mechanisms intended to strengthen transparency and accountability. Increasingly, as part of their open development efforts, international development 
organisations, NGOs and funders are being encouraged to publish the data that they hold as open data, and many have signed up to do so through the International Aid Transparency Initiative. In theory institutions and governments making their data open can improve the sector, making it easier to reduce corruption, follow financial flows, organise resources better, and more generally level the 'information playing field'.

Although this data availability is a necessary step towards demanding accountability from powerful actors, it is not the only step. Especially within international development, many more steps are required to truly empower the actors involved to actually understand more about the world around them, and make use of the data that affects and relates to them.

Different actors involved in international development projects have different information needs: from taxpayers in donor countries who want to know what their tax dollars are funding, to project managers who want to be able to track their project over a period of time, to the people who are actually affected by the project and want to know what is going on.

One assumption that is often made is that simply publishing open data in international development is a tool of accountability for all; but this generalised framing of the target audience is counter-productive. Through my work on the Open Development Toolkit, I spent time looking at ways in which international development actors are making their data available and it become clear that those who try to please everyone, end up pleasing nobody. International development organisations that have developed online data portals aimed at 'the general public' have ended up with largely unusable tools. Not all of them are like this, though: the best example I have seen of a concretely useful tool is the UK's DevTracker tool (see Figure 1), which tracks UK-funded international development projects abroad. In this case, the creators were very clear about their target audience: UK taxpayers. The tool helps this cohort to easily and clearly see where UK aid funds are going and on what, and are therefore able to better engage with where their tax money is going.

DevTracker's efforts at identifying and meeting the needs of their target audience have been largely successful, but this is one example among many other less useful portals. These cases show that despite commendable efforts from open data advocates within international organisations, if the tool does not meet the needs of groups, the tool itself is, sadly, largely a waste of resources. 


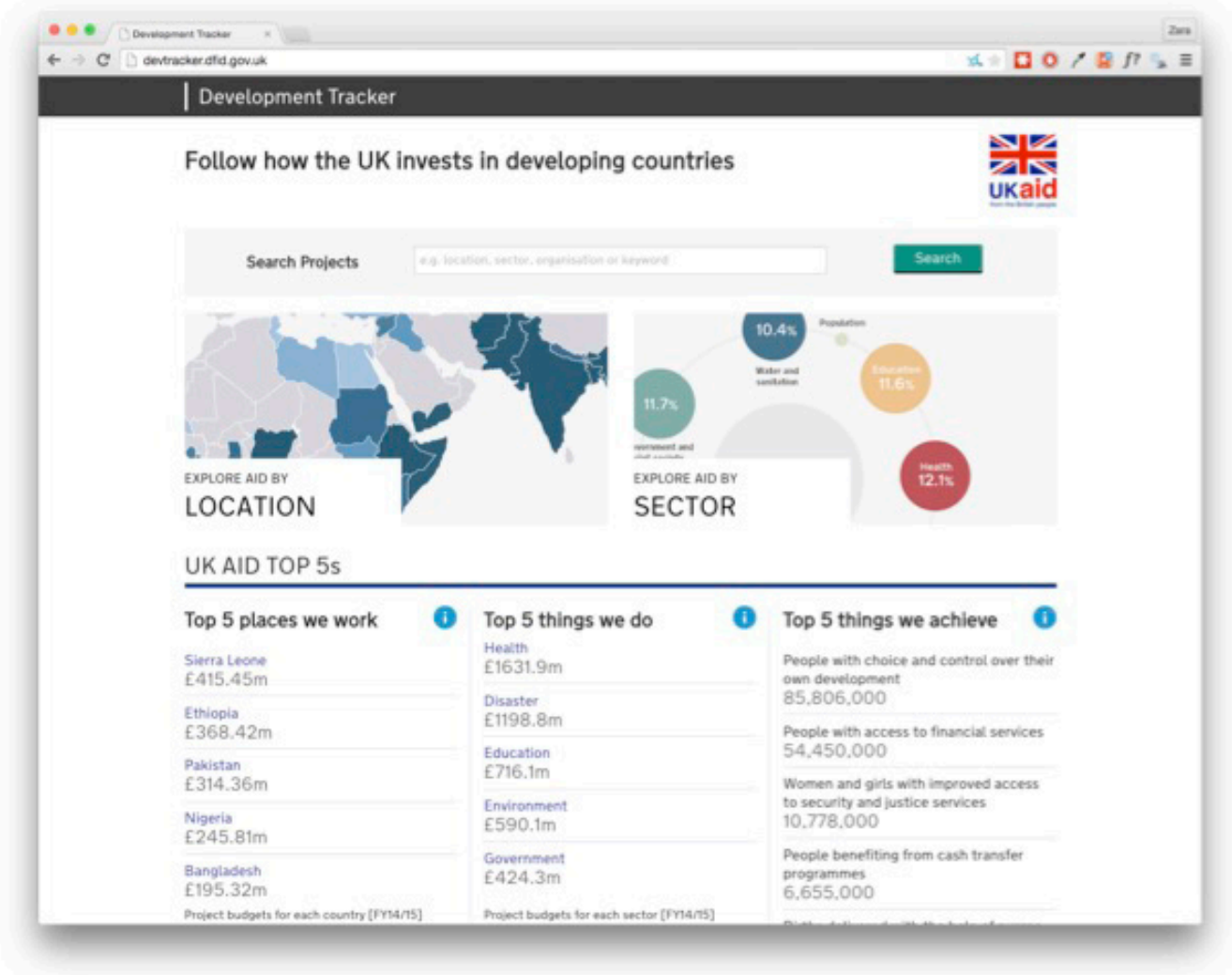

Figure 1: screenshot of the DevTracker site

It is also clear that online open data efforts are not sufficient if the information does not reach the people most affected by development projects. For example, the prerequisites to being able to access online open data include, but are not limited to: being able to read; Internet access; being technically literate enough to be able to find the data; being data literate enough to understand what the data might, or might not, be revealing; and, having the time and interest to actually go through the process of accessing and analysing the data.

As you can imagine, very few of these conditions apply to many of the people who are affected by international development projects. This leads us to think about offline open data and the importance of information intermediaries, also known as 'infomediaries'. Offline open data can get data to people who don't have access to the Internet, or any sort of device: examples include budget data being written up on chalkboards in town centres, or printing out maps and asking people with knowledge of the area in question to confirm the existence of landmarks released through open data initiatives, as is happening in Chennai, India. Infomediaries, such as the 'infoladies'-women who cycle around villages in Bangladesh with portable devices, offering access to the Internet-or data journalists 
who use online data to tell compelling stories, which can reach 'offline communities' in Cambodia, are both examples of how online open data can reach people who don't meet the conditions outlined above.

While online open data portals and tools are useful for those living in relative privilege to understand what their governments are doing, for open data to reach its full potential, projects such as these, which look first at how project beneficiaries already access information, and then use these established channels to communicate, are imperative.

\section{Open sourcing the tools developed}

Technology has played a very significant role in the Open Development movement, and many organisations in the sector are embracing technological tools in their effort to enhance the sector. However, very few of these code-based tools are open source, despite many of them being publicly funded. My work in this field has led to a realisation that the reason behind this wasn't-as we had first thought-purely due to a lack of awareness, but because of a lack of understanding regarding the value and culture of open source.

One example can be seen in the tool mentioned above, DevTracker. While the code behind this tool is open source, it has yet to actually be used by any other agency; from what I learned through speaking to various organisations, the idea of using that code is somehow understood as 'copying' someone else's work. Organisations seem reluctant to use tools that have been developed by others, wanting instead to develop their own versions, resulting in tool duplication and wasted resources.

Through this example we see that despite organisations in the sector having the good intentions of wanting to engage in Open Development, a lack of holistic understanding of the movement or a lack of coherency in terms of the values driving it means that the resulting impact is greatly reduced. Their 'open' intentions manifested themselves in wanting to share data online in a useable format, via a web based portal; however their traditional approaches to technology development led to a reluctance to contribute to open source projects started by others. Here, the desires of open data activists to have the data, seems somehow to have missed the more technical nuance of open sourcing the code. 


\section{Innovation versus 'conservativeness', and doing it responsibly}

The new technologies we have in place-whether those used to publish open data or those used as part of open data programs themselves-are opening up new ethical issues for international development programmes. As part of the Responsible Data series, I joined a group of people in September 2015 to co-author a book on "responsible data in international development".

While the forum the book emerged from examined many different issues, one broad trend emerged: the priority given to speed in engaging with new technology. Organisations who are jumping on the latest innovation are perceived to be displaying a progressive attitude, considered a desirable quality. Organisations who don't do so, are considered to be old-fashioned and resistant to change.

Within this perspective, the value proposition we are creating around the use of technology in development centres around the speed with which new technologies are being implemented; the faster, the better. But this ignores the need for some crucial first questions-is the technology ready for use in the real world? Is it appropriate in this context? Is this the best use of limited resources?

A concrete example of this might be when decisions are made about development data that will be published as open data, and the data that will be kept internal within an organisation. This decision is largely put in the hands of the donor rather than with the local development organisations or actors who may be most affected by publishing the data. Taking the time to ask various stakeholders within the receiving NGO or institution whether publishing potentially sensitive data is suitable or not, takes time and resourcesbut it is wholly necessary to prevent any unintended harm being done.

Taking time for self-examination, and investing more time and money into research, should not be considered a sign that an organisation is old-fashioned or unwilling to make the most of new technologies: it could in fact provide evidence that they are taking a more responsible and aware perspective towards new technologies and are willing to really invest in making sure that they are choosing the most appropriate systems.

Unlike in many other communities so closely related to technology, such as the startup 
sector, where iteration and a fast turnaround are top priorities, in the realm of international development, implementing new approaches that have not been tested or deemed appropriate can have far-reaching and damaging effects on already vulnerable people and communities. This is true in relation to any development intervention, but given the remarkably new ground that the employment of technology in open development is treading, the field deserves particular attention and caution.

\section{A case for thoughtful Open Development}

There are a number of potential issues to be aware of within the Open Development movement. These include challenges regarding effective and useful publishing of open data-whether that be online, or offline, or the open source development of new tools-and the new responsibilities that aid organisations face with regards to innovation and new technologies in the sector.

The Open Development movement is, as mentioned, relatively new, and it is highly likely that more ethical issues will come to the fore as the movement evolves in the future. However, if done correctly, it is clear that embracing a more open way of working within the sector has the potential to have a positive impact in many ways. Taking a critical view of open development, and open data, is by no means a rebuttal to the potentially life-changing and hugely positive effect that this could have on the lives of millions around the world; it is more an effort to make sure that these efforts and resources are carried out in the most effective and useful way possible, especially keeping the needs of those that we are trying to help at the forefront of our minds.

\section{Biographical Note}

Zara Rahman is Research Coordinator at the engine room, an NGO that work on supporting the use of technology in advocacy. Her research focuses on the intersection of international development, technology and accountability, and in September 2014, she co-authored a book on the responsible use of data in international development, which is available for download here. Zara is a Fellow at the Centre for Internet \& Human Rights at European University Viadrina, and is based in Berlin, Germany. 


\section{References}

Smith, Matthew and Laurent Elder. 'Open ICT Ecosystems Transforming the Developing World', Information Technologies and International Development Journal 6.1 (2010): 65-71.

The LOCKSS System has the permission to collect, preserve and serve this open access Archival Unit

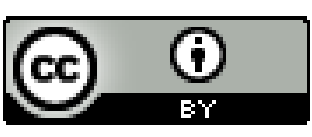

This Isuue of the Fibreculture Journal by The Fibreculture Journal Incorporated is licensed under a Creative Commons Attribution 4.0 International License.

\section{OPEN HUMANITIES PRESS}

The Fibreculture Journal is published by The Fibreculture Journal Incorporated in partnership with Open Humanities Press. 


\section{The Fibreculture Journal}

DIGITAL MEDIA + NETWORKS + TRANSDISCIPLINARY CRITIQUE

issue 26: Entanglements - Activish and Jechrology

FCJMESH-O11 'We don't work with video, we work with People':
Reflections on Participatory Video Activism in Indonesia

M. Zamzam Fauzanafi

Kampung Halaman

In this article Indonesian visual anthropologist and co-founder of Kampung Halaman, Zamzam Fauzanafi, reflects on what he has learnt through almost a decade's experience of using video technologies to support grassroots activism. He argues that while technologies will always change, what matters is a deep understanding of how they help or hinder your capacity to work with the people your work is meant to support.

A different nature opens itself to the camera than opens to the naked eye-if only because an unconsciously penetrated space is substituted for a space consciously explored by man. (Walter Benjamin [1936], 1968: 236).

doi: $10.15307 / f c j . m e s h .011 .2015$

In 1993 in Indonesia-long before I started to develop a participatory video program with the not-for-profit organisation Kampung Halaman-l learnt about Roem Topatimasang, who was using participatory tools for community empowerment in the small and remote Kei Islands, southeast of Moluccas. He supported the villagers to make a video called, Buka Sasi Lompa, about the revitalisation of traditional practices to address and protect the sea from overfishing. 
At that time, I was just a high school student from West Java who really loved watching movies. The flickering images and thundering sound of Hollywood and Hong Kong's Kung fu movies have been a welcome distraction to me ever since. I didn't think much about how audiovisual technologies affected us though, until later when I read about Walter Benjamin's concept of “distraction” ([1936], 1968:240). This term describes how films can make us lose ourselves in a dreamlike world, but can also re-engage us by pulling us out of our everyday experience. This contributes to our experience of film as an oscillation between critical distance and participatory immersion.

In 2006, inspired by the work of Roem Topatimasang and the Kei Islands villagers, I helped set up an organisation to help young Indonesians make their own videos. At age 28 , I drew from my experience as a community organiser, and what I had learned while completing a master's degree in visual anthropology.

Our idea was to use video as a participatory method to support young people to recognise and reflect on the condition of self, community, and their local environment. We called our organisation Kampung Halaman (Homeland). Our name was chosen to signify our philosophy that by making their own videos, young people could re-engage with their homeland, after their distraction from films, videos, or TV shows that were always produced by other people, in other places. This was connected to our belief that while TV shows and films often encourage us to build new dreams, these dreams are always detached from the realities of home, distancing us from our actual environment, effacing our sensitivity and attachment to our communities, rendering us remote from and powerless to change the places we live in.

In practice, our interventions were mostly focused on handing a camera (video or still) to the young people who nominated themselves to participate in our program. Seeing and exploring their environments, everyday activities, social and cultural process through a camera helped reveal aspects of reality that they may have registered in their senses but had never quite managed to process consciously. In this way, we found that young people could use a camera to try to recognise the problems and potential in their lives, communities, and surroundings.

We worked with young people from different communities in many parts of Indonesia. While each community we worked in is unique, they shared a common feature: what we call, "communities in transition." These are communities who are undergoing rapid environmental, economic, social, and cultural changes. We believe that introducing participatory video to such communities supports them to record and reflect what is happening but also to become active participants of change. 
In the eight years since we began, we have tried, tested and developed a number of different approaches for "doing" participatory video. In the rest of this article I will reflect on some of these approaches, in order to share what l've learnt about the frictions that can emerge between the needs and philosophies of grassroots activism and the philosophies embedded in video, and increasingly also in mobile and Internet technologies.

In our first project in 2006 we worked with young people from a small village just outside of Yogyakarta that was devastated by an earthquake that had ruined almost $80 \%$ of homes and had taken the lives of thirteen villagers. In addition to this catastrophic disaster there were other tensions and problems in the village. The ongoing disappearance of the paddy fields and the subsequent loss of agricultural work and employment and a growing religious radicalisation also contributed to the context for our participatory video program. Our model saw a facilitator live for almost one year in the village, as part of a two-year program, focusing as much on the process of video-making and on the discussions that emerged around filming and screenings as on the videos themselves. In this way, video was used to map out problems and as a vehicle to start discussions about possible solutions from and within the community.

From this approach and experience we learnt that video was really effective in that it enticed young people to participate, and to then become involved in mapping out problems and engaging in discussions. However, we needed other approaches to support young people and other members of the community to work together and organise themselves in ways that could help them to actually address the problems they identified. Moreover, we found only one or two young people were interested in mastering the technology, and this created 'specialisation' or even 'professionalisation' in video making, which was not what the program had intended. This meant just a few people tended to take responsibility for the camera, which took away some of the planned benefits of having people see things in a different way. However, other members of the community still gain these benefits when they became spectators. Therefore, even though only a small number of young people might make the videos, in the end this can result in more people seeing things in a different way which then triggers new discussions among members of community.

Our next project occurred one year later and involved working with young people from the semi-urban provincial city, Tasikmalaya, in West Java. Here we tried to involve young people from very different backgrounds-racial, ethnic, religious, economic, and educational-to participate in a photo and video participatory program that we developed with a local media center. The result was a two-year program that involved hundreds of young people. To cope with the large numbers of participants, we divided them into small 
groups based on their community and locality. Working with facilitators, this model lent itself to a more advocacy-focused approach to video-making since this approach was able to support young people to move from identifying issues to developing campaign videos that identified pathways for change.

The videos that the young people created looked at issues including multiculturalism, un-employment, environmental degradation, education, economic disparity, and a lack of public space. The videos also explored different styles of representation based on young people's interests, backgrounds, and hobbies. They were sometimes poetic, musical, theatrical, or journalistic. However, later, when we tried to bring the different groups of young people together to continue their work at a local media center, we found these differences became an impediment. Young people saw things differently and many did not want to work with one another. We concluded that the local media center idea didn't work as most participants stopped making videos.

Despite this, a few of these participants have now developed their own participatory video program with youth from different communities. They even created their own local annual film festival. This made us question what long-term success really looked like. Should we expect more than a few young people to continue working with video and communitybased work beyond our program?

At times we experimented with more 'freestyle' approaches to participatory video making with young people from different traditional art communities in Yogyakarta, Bali, and Ponorogo (East Java). We combined audio-visual media with local, traditional performing arts to offer different approaches to community organising, campaigning, and empowerment. This method resulted in surprising outcomes such as the re-engagement of young people with traditional arts and the transformation of local traditional arts groups that in turn supported young people to become more active participants and sometimes instigators of change.

What we learnt from this approach was that video could become creatively entangled with traditional creative expression to create new modes of performance: where participants' bodies revealed new stories. The camera became a 'mimetic machine' or 'sensuous knowing machine' that was able to reconnect us (Taussig, $1993: 23$ ). Participants in Ponorogo, who are also traditional dancers, used the words "ngedan"* or "trance" to describe how they would engage in making video and dancing. By "ngedan" *they perceive their surrounding differently and wake up with new fresh insight. 
Most recently, following the rise of the Internet and in an attempt reach broader participants and audiences, we developed a more networked, less locally focused approach for making and distributing participatory video. These projects are shorter but involve many youth communities, local organisations, and NGOs from all around Indonesia. We make video campaigns on different issues relevant to young people in Indonesia such as education, poverty, environment, health, pluralism and violence. Videos that serve as a tool for advocacy are distributed online and they are also screened offline in different communities all around Indonesia on the 12th of August to commemorate International Youth Day. With this approach we have learnt that with networked technologies it is possible to support young people to talk about problems at a regional or national level and to do this in a way that connects them and builds a network. Young people in Indonesia can learn from one other's videos about different, but also similar issues and they can use these to discuss possible solutions.

Our experiences over the past eight years have taught us some broad lessons about the use of video technologies as participatory tools for therapy, advocacy, and empowerment. For example, a technology such as video has its own affordances that make certain actions possible and others not. Video might open up the optical unconscious and offer new possibilities for exploring reality; but organising, building, and sustaining a community to take action and implement changes is a more challenging story. We've learnt that social change action is afforded less by any technology as it is by the human interactions and deliberations that emerge because of the way we work.

We have also learned that fetishising technologies as powerful tools for advocacy and empowerment can hinder tactical usages of technology. Many times we have seen that it's not the video that has mattered but our participatory video approach: the videos have functioned as a means of knowing, reflecting, and sharing. Our approach has encouraged young people to express themselves, to develop a critical awareness of the problems they face, and to trigger further actions that seek to deal with the problems identified through the video-making process. Video is not a technology that is intrinsically empowering; it is just one tool that opens new possibilities for participation and empowerment.

Another realisation has been the worrying trend to measure the effects of the use of participatory video by metrics: through counting the number of participants and the audiences reached. This method is tempting but ultimately misleading and damaging. If it were applied to past projects it would misrepresent the impact our projects have had. We believe it is crucial to scratch beyond the surface of numbers to develop a critical assessment of participatory video effects that is based on appropriate forms of evidence and on the specific context. 
Ultimately, I have learnt that we don't work with video, we work with people. We have used video at Kampung Halaman because it has helped us to work with people in different ways: by allowing new ways of seeing, by facilitating conversations and by allowing stories and messages to spread further. But this only works if we understand different contexts and opportunities and pursue participatory approaches that ensure technologies are in the control of people who can benefit most from social change.

\section{Author Biography}

M. Zamzam Fauzanafi is a visual anthropologist and activist. He was the founder of Kampung Halaman Foundation and worked with them 2006-2012. Zamzam is now a lecturer at the Department of Anthropology, Gadjah Mada University, Yogyakarta where he supports students to work with participatory video and with Kampung Halaman. He is currently completing a PhD on the 'Digital Act of Citizenship' with Leiden University, The Netherlands.

\section{References}

Benjamin, Walter. "The Work of Art in the Age of Mechanical Reproduction", in Hannah Arendt (ed.) Illuminations (New York: Schocken Books, 1968), 217-251.

Taussig. Michael. Mimesis And Alterity; A Particular History of The Senses (New York, London: Routledge, 1993). 


\section{OPEN HUMANITIES PRESS}

The Fibreculture Journal is published by The Fibreculture Journal Incorporated in partnership with Open Humanities Press. 